\title{
Impact of Monoenergetic Photon Sources on Nonproliferation Applications
}

Cameron Geddes, Bernhard Ludewigt, John Valentine, Brian Quiter, Marie-Anne Descalle, Glen Warren, Matt Kinlaw, Scott Thompson, David Chichester, Cameron Miller, Sara Pozzi

March 2017

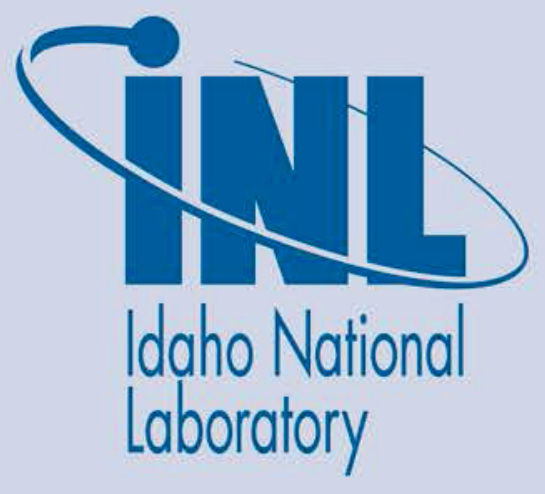

The INL is a U.S. Department of Energy National Laboratory operated by Battelle Energy Alliance 
INL/EXT-17-41137

\section{Impact of Monoenergetic Photon Sources on Nonproliferation Applications}

Cameron Geddes, Bernhard Ludewigt, John Valentine, Brian Quiter, Marie-Anne Descalle, Glen Warren, Matt Kinlaw, Scott Thompson, David Chichester, Cameron Miller, Sara Pozzi

March 2017

Idaho National Laboratory
Idaho Falls, Idaho 83415

http://www.inl.gov

Prepared for the

U.S. Department of Energy

Assistant Secretary for _, OR Office of

Under DOE Idaho Operations Office

Contract DE-AC07-05ID14517 


\title{
Impact of Monoenergetic Photon Sources on Nonproliferation Applications
}

\author{
Final Report
}

January 31, 2017

\author{
Project Team \\ Cameron Geddes (PI), Lawrence Berkeley National Laboratory \\ Bernhard Ludewigt (Co-PI), Lawrence Berkeley National Laboratory \\ John Valentine, Lawrence Berkeley National Laboratory \\ Brian Quiter, Lawrence Berkeley National Laboratory \\ Marie-Anne Descalle, Lawrence Livermore National Laboratory \\ Glen Warren, Pacific Northwest National Laboratory \\ Matt Kinlaw, Idaho National Laboratory \\ Scott Thompson, Idaho National Laboratory \\ David Chichester, Idaho National Laboratory \\ Cameron Miller, University of Michigan \\ Sara Pozzi, University of Michigan
}

Prepared by:

Lawrence Berkeley National Laboratory

Prepared for:

DOE NNSA, DNN R\&D Enabling Capabilities

Program Manager: James Peltz

Project Number: LB15-V-LB-Monoenergetic Photons-PD3UE

INL/EXT-17-41137

Final Report: Project LB15-V-LB-Monoenergetic Photons-PD3UE. 


\section{Executive Summary}

Near-monoenergetic photon sources (MPSs) have the potential to improve sensitivity at greatly reduced dose in existing applications and enable new capabilities in other applications, particularly where passive signatures do not penetrate or are insufficiently accurate. MPS advantages include the ability to select energy, energy spread, flux, and pulse structures to deliver only the photons needed for the application, while suppressing extraneous dose and background. Some MPSs also offer narrow angular divergence photon beams, which can be used to target dose and/or mitigate scattering contributions to image contrast degradation. Current bremsstrahlung photon sources (e.g., linacs and betatrons) produce photons over a broad range of energies, thus delivering unnecessary dose that in some cases also interferes with the signature to be detected and/or restricts operations. Current sources must be collimated (reducing flux) to generate narrow divergence beams. While MPSs can in principle resolve these issues, they remain at a relatively low Technology Readiness Level (TRL). Candidate MPS technologies for nonproliferation applications are now being developed, each of which has different properties (e.g., broad vs. narrow angular divergence). Within each technology, source parameters trade off against one another (e.g., flux vs. energy spread), representing a large operation space. This report describes a survey of potential applications, identification of high priority applications, and detailed simulations addressing those priority applications. Requirements were derived for each priority application, and analysis and simulations were conducted to define MPS parameters that deliver benefit. The results can inform targeting of MPS development to deliver strong impact relative to current systems.

A prioritized assessment is presented of a range of nonproliferation applications where monoenergetic photon sources (MPSs) may enable new capabilities or significant performance enhancement (Table 1). A broad set of applications was identified by our team, other national laboratory subject-matter experts, and US government agencies responsible for related operations (e.g., Department of Energy Defense Nuclear Nonproliferation R\&D stakeholders) [1]. Based on the initial assessment, applications prioritized for detailed analysis were: cargo screening and interdiction of Special Nuclear Materials (SNM), detection of hidden SNM, treaty/dismantlement verification, and spent fuel dry storage cask content verification. High resolution imaging for stockpile stewardship was considered as a sub-area of the treaty topic, as it is also of interest for future treaty use (e.g., for uniquely identifying individual weapons). Discussions with DNN R\&D and their stakeholders, and assessment of prior studies, identified these areas as of strong interest for nonproliferation. Preliminary analysis indicated that there is significant potential benefit for each of these applications, and that the photon source requirements are likely within range of realistic MPS candidates. Additional applications were also identified (Table 1) which were addressed to the extent practical by leveraging overlap with the main topics. In particular, strong emergency response capabilities are indicated by the cargo, treaty and stockpile stewardship sections. Similarly, the potential of MPSs with small photon emission spot size (e.g., Thomson sources) to enable high-resolution radiography with reduced dose indicates strong potential impact on areas outside nonproliferation, such as medical imaging and industrial nondestructive analysis. Preliminary analysis only was conducted for photonuclear data collection, spent fuel assembly assay, materials characterization in accident scenarios, waste drum content analysis, nuclear fuel quality control, and pyroprocessing monitoring. These are possible topics for future work. No significant MPS benefit was identified for applications to safeguards $\mathrm{UF}_{6}$ cylinder enrichment verification, or to nuclear forensics. 
Table 1: Prioritized potential nonproliferation applications.*

\begin{tabular}{|c|c|c|c|}
\hline Application & Potential Impact & \begin{tabular}{|l|}
$\begin{array}{l}\text { Stakeholder } \\
\text { Priority }\end{array}$ \\
\end{tabular} & \begin{tabular}{|l|} 
MPS Study \\
Priority
\end{tabular} \\
\hline $\begin{array}{l}\text { Screening and interdiction } \\
\text { Included: screening material } \\
\text { in/out of nuclear facilities }\end{array}$ & $\begin{array}{l}\text { SNM, explosive, contraband detection at } \\
\text { lower dose. Radiography with higher } \\
\text { spatial \& Z resolution, higher contrast. } \\
\text { Photofission at } \leq 10 \mathrm{MeV} \text {; enable NRF }\end{array}$ & High & $\begin{array}{l}\text { High: detailed } \\
\text { simulations } \\
\text { conducted. }\end{array}$ \\
\hline Detection of hidden SNM & $\begin{array}{l}\text { SNM Detection at lower doses, larger } \\
\text { distances, or behind thicker shielding }\end{array}$ & High & $\begin{array}{l}\text { High: detailed } \\
\text { simulations } \\
\text { conducted. }\end{array}$ \\
\hline $\begin{array}{l}\text { Treaty/Dismantlement } \\
\text { Verification } \\
\text { Included: Stockpile \& dummy } \\
\text { verification }\end{array}$ & $\begin{array}{l}\text { Warhead confirmation, unique identifier of } \\
\text { warhead and components, dismantlement } \\
\text { confirmation. Lower priority: items in } \\
\text { containers. }\end{array}$ & High & $\begin{array}{l}\text { High: detailed } \\
\text { simulations } \\
\text { conducted. }\end{array}$ \\
\hline $\begin{array}{l}\text { Safeguards: Dry-storage cask } \\
\text { verification }\end{array}$ & $\begin{array}{l}\text { Determination of assembly basket } \\
\text { occupancy }\end{array}$ & High & $\begin{array}{l}\text { Medium: detailed } \\
\text { simulations } \\
\text { conducted. }\end{array}$ \\
\hline Emergency response & $\begin{array}{l}\text { Similar to screening. Added benefit from } \\
<\text { mm spatial resolution. Small MPSs are } \\
\text { important. Potential multiple applications } \\
\text { of laser-driven MPS (e.g., laser cutting). }\end{array}$ & $\begin{array}{l}\text { Medium to } \\
\text { High** }^{* *}\end{array}$ & $\begin{array}{l}\text { Medium: future } \\
\text { study possibility }\end{array}$ \\
\hline Photonuclear data & $\begin{array}{l}\text { Measurements at nuclear laboratories, } \\
\text { instead of fixed facility. }\end{array}$ & Medium** & $\begin{array}{l}\text { Medium: future } \\
\text { study }\end{array}$ \\
\hline $\begin{array}{l}\text { Safeguards: SNF assembly } \\
\text { assay }\end{array}$ & $\begin{array}{l}\text { Reestablish inventory after loss of CoK; } \\
\text { "fingerprint" Pu content; detect } \\
\text { inconsistency w/ declared operator history. }\end{array}$ & Low & Low priority \\
\hline \begin{tabular}{|l} 
Safeguards: Materials \\
Characterization in Accident \\
Scenarios. Related: waste, \\
fresh fuel characterization
\end{tabular} & $\begin{array}{l}\text { Measure Pu isotopic content in } \\
\text { damaged/melted fuel, or in waste. }\end{array}$ & Low & Low priority \\
\hline $\begin{array}{l}\text { Safeguards: Accountancy and } \\
\text { Pu Monitoring in } \\
\text { Pyroprocessing }\end{array}$ & $\begin{array}{l}\text { Measure Pu in spent fuel before dissolution, } \\
\text { monitor Pu in refiner vessel, assay isotopics } \\
\text { of product and waste. }\end{array}$ & 1, Low & Low priority \\
\hline $\begin{array}{l}\text { Safeguards: Fresh fuel } \\
\text { verification in transport } \\
\text { containers }\end{array}$ & $\begin{array}{l}\text { MPS-based system capable of verification, } \\
\text { but current research may lead to simpler } \\
\text { solutions }\end{array}$ & Medium & $\begin{array}{l}\text { Low: other } \\
\text { solution likely. }\end{array}$ \\
\hline $\begin{array}{l}\text { Safeguards: } \mathrm{UF}_{6} \text { cylinder } \\
\text { enrichment verification }\end{array}$ & No capability enhancement identified & High & None \\
\hline Nuclear forensics & No capability enhancement identified & Low & None \\
\hline
\end{tabular}

* Applications outside nonproliferation are listed in table where related. Additionally, medical radiography and industrial NDA would benefit from increased penetration/resolution and reduced dose.

${ }^{* *}$ Tentative assessment based on informal discussions.

Higher-fidelity calculations and modeling results were conducted that allowed quantitative evaluation of the prioritized applications and derivation of the key MPS properties that drive application benefit. Full system performance was considered to evaluate the impact that monoenergetic sources would have on each prioritized application. This included natural and induced background radiation, beam propagation to a target, penetration, signature generation, detection sensitivity, and radiation dose to target and operators. Performance of potential monoenergetic photon sources was evaluated, including source technology tradeoffs and constraints. Simulations focused on the conventional signatures of radiography, photofission, and Nuclear Resonance Fluorescence (NRF) to enable comparison to present methods and evaluation of benefit. 


\section{Cargo Screening and Interdiction of Shielded SNM}

Screening for and interdiction of SNM, typically in cargo containers, train cars and trucks, is a priority application for the Department of Homeland Security, as well as other agencies (e.g., the Department of Energy Nuclear Smuggling Detection and Deterrence program-formerly Second Line of Defense). Access to both sides of the object is typically available, which facilitates use of radiography as a primary screening method, including use of dual-energy transmission ratios to resolve the $\mathrm{Z}$ of objects. Photofission and NRF are considered as secondary screening methods to clear alarms (i.e., to resolve whether or not an object is SNM).

Significant potential benefit was indicated for the configurations analyzed for radiography applications using monoenergetic photon beams. Replacing bremsstrahlung with a monoenergetic source having similar emission angle and pulse structure enables reductions in radiography dose by factors of 2 to 4 . Additionally, MPSs with narrow angular divergence (e.g., Thomson sources) can deliver small beam diameter at the target without severe collimation that would throw away almost all of the flux. Narrow-divergence beams-at the few mrad level to deliver cm-size spots on target-nearly eliminate image contrast degradation due to scattering. Scattering is severe for many cargo cases, and mitigation can therefore either increase image quality or provide a significant added reduction in dose for the same image quality. Dose reduction due to mitigation of scattering is in the range of 3-4x even compared to very narrowly collimated fan bremsstrahlung systems (and has a much greater advantage vs. broadly collimated sources). To achieve high image quality in the presence of scattering, narrow divergence was found to be more important than control of energy spectrum. This indicates that MPSs with narrower angle emission than bremsstrahlung fans will provide strongest advantage (and correspondingly, that MPSs with broader collimated emission angles than those of bremsstrahlung systems may not be advantageous in cases where scattering is significant). Narrow divergence also enables pencil beam scanning, with the advantages of spot-by-spot dose modulation. Since most of a cargo is typically low density, with isolated high-density areas (e.g., an engine or machine part), such dose adaptation strongly reduces dose to all but suspect region. Moderately narrow energy spread, at the $20 \%$ level, is on the other hand important to using dual energy transmission ratios to identify regions containing different Zs. Contrast between regions of differing $\mathrm{Z}$ is drastically increased by using a monoenergetic source, by factors of a few to ten in the examples examined (depending on configuration). This could enable effective discrimination in cases that are not currently accessible. Overall reduction in dose to stowaways and in scatter dose to surroundings and exclusion zones could be more than an order of magnitude, depending on cargo and system design. Reduced dose could ease operational constraints, or could be used to penetrate thicker cargo within prescribed dose limits.

MPSs for radiography should produce photons at selected energies from 3-9 MeV, and with typical fluxes on target (after collimation, determined by scan rate and penetration needs) in the range of $10^{10}$ photons per second for initial capabilities to $10^{12}$ photons per second for high performance applications. Pulsed sources should have repetition rates in the $\mathrm{kHz}$ range to enable initial scanning capabilities, while repetition rates in the $10-50 \mathrm{kHz}$ range would enable high performance. For example, fluxes of $\leq 10^{8}$ photons/shot at repetition rates of $5-50 \mathrm{kHz}$ are suitable for scanning containers at $0.1-1 \mathrm{~m} / \mathrm{s}$ while penetrating thicknesses of $\sim 40 \mathrm{~cm}$ of steel, using pencil-beam sizes of $1-4 \mathrm{~cm}^{2}$.

A key advantage of MPSs for clearing an alarm or detecting SNM in cargo screening is the capability to induce a much stronger photofission signal for a given dose than other sources. For example, for a $9 \mathrm{MeV}$ MPS, the dose to cargo and surroundings per fission induced in $2 \mathrm{~kg}$ of HEU behind $20 \mathrm{~cm}$ steel is more than $50 \times$ lower than for a $9 \mathrm{MeV}$ bremsstrahlung source due to a 
combination of reduced beam attenuation in the steel and higher photofission rates. Narrow divergence MPSs (e.g., Thomson sources) are particularly well suited for resolving an alarm caused by an object in an identified location because the low-divergence beam can deliver a high photon flux to that area. Identification of $2 \mathrm{~kg} H E U$ behind $30 \mathrm{~cm}$ of shielding in seconds is realistic using an MPS with $\leq 10 \mathrm{mrad}$ divergence operating at $1 \mathrm{kHz}$ and delivering $10^{8}$ photons/shot on target (after collimation) at $9 \mathrm{MeV}$ with $10 \%$ energy spread. NRF is enabled at energy spreads of approximately $2 \%$, and repetition rates of $10 \mathrm{kHz}$ (realistic goals for Thomson sources) would make it possible to detect $2 \mathrm{~kg}$ HEU inside $10 \mathrm{~cm}$ thick steel shielding in tens of seconds. Future Thomson sources with an energy spread of a fraction of a percent may become available; that would greatly increase capability. In general, NRF is more suitable where composition analysis is needed, either for contraband detection or isotopics of SNM.

Cargo screening is an application where MPSs could have high impact. Benefits for radiography combine the effects of monoenergetic transmission and up to a tenfold increase in $\mathrm{Z}$ contrast. Narrow-divergence MPSs add scattering mitigation, and dose adaptation. A narrowdivergence MPS could therefore enable superior resolution combined with typical dose reductions of one to two orders of magnitude. Alarm resolution using photofission and NRF is enabled with orders-of-magnitude lower doses and with increased performance.

\section{Detection of Hidden SNM}

Detecting hidden SNM was considered separately from screening in order to address scenarios where access to the object/container of interest is limited to one side (at most, $180^{\circ}$ ). These scenarios are of interest to DNDO for parked vehicles, to emergency response, and potentially to the Defense Threat Reduction Agency. A constrained inspection volume is assumed to have been pre-defined (e.g., a vehicle of interest at distances of up to tens of meters). Larger distances or objects (i.e., standoff, broad area scans) were excluded based on preliminary calculations. Photofission is an appropriate method for one-sided access. For clearing an object, sufficient information about the amount of shielding surrounding the nuclear material must be available to firmly conclude that a lack of observed fission signatures is not due to shielding but proves the absence of SNM.

MPSs can significantly reduce doses to the object for photofission by delivering all of the photons in the range of high fission cross section. The strongest photofission signal is obtained near the photofission resonance energy of $\sim 15 \mathrm{MeV}$. The imparted doses are approximately a factor of 4 lower for MPSs operating near the resonance energy than for a bremsstrahlung source (19 MeV endpoint energy). Most importantly, energies $<10 \mathrm{MeV}$ avoid production of most photoneutrons, expediting use of the strong prompt neutron signature, which was the strongest fission signature for the cases studied. At such energies the MPS dose advantage can exceed a factor of 50. Simulations evaluated use of various photofission signatures to detect $25 \mathrm{~kg}$ and 18 kg HEU objects in a variety of shielding configurations including water, steel and composite B-poly/lead of varying thicknesses. Sources with narrow angular divergence $(\sim$ mrad, so that the photon-beam spot size on target is comparable to or smaller than the SNM) along with intense beams (sufficient to generate detected photofission signals well above background), are important to reduce overall dose to the object and environment.

Operating an MPS at $13.1 \pm 25 \% \mathrm{MeV}\left(8.5 \times 10^{8}\right.$ photons per source pulse on target, after collimation) for delayed signatures and $6.7 \pm 20.5 \% \mathrm{MeV}\left(6.9 \times 10^{8}\right.$ photons per source pulse $)$ for prompt neutrons maximizes fission production without generating problematic non-negligible levels of source-induced background or signature interferences. Positively identifying the presence of fissionable material was shown to be realistic for the configurations analyzed in minutes, at ranges of $20 \mathrm{~m}$ and through shielding thicknesses up to $30 \mathrm{~cm}$ using a source 
producing $\sim 10^{12}$ photons/second within a few-mrad emission angle. Scaling indicates that use of shorter distances and/or higher fluxes may realistically extend the shielding thickness that can be penetrated to the range of $40-50 \mathrm{~cm}$ of steel.

The results inform the size of a shielded object within which absence could be determined, and indicate that such a measurement could be realistic in objects at the $\sim 1 \mathrm{~m}^{3}$ size range using a narrow-divergence MPS. Even with such a source, localization and coarse size determination of a shielded object of interest is crucial to allow determination of absence. An appropriate backscatter measurement may expedite measurements by identifying high-Z or high-density areas, consequently localizing a region of interest.

\section{Treaty/Dismantlement Verification and Stockpile Stewardship}

Verification of nuclear warheads and their dismantlement is a high priority for treaty verification. Both scattering and transmission NRF are promising techniques for measuring material characteristics in the context of treaty verification, and MPS-based photon sources provide an order-of-magnitude or more performance improvement over bremsstrahlung-based sources. NRF scattering generally performs better than NRF transmission in this application. Using a photon source operating at $10 \mathrm{kHz}$ with a 2\% FWHM energy distribution, detection of quantities of interest of $\mathrm{HEU}, \mathrm{Pu}$, and $\mathrm{HE}$ are possible in times typically of a few minutes. The required photon intensity is relatively modest at less than $2 \times 10^{6} \gamma /$ shot on target (after collimation). The primary limitation to reducing the time-to-detection/confirmation is the total rate on the detector; if the total rate is too high, pileup will occur, obscuring the signal. Evaluation of different filtering options in front of the detector may reveal a way to improve that situation. Alternatively, if one could develop a fast threshold counting detector, it may be possible to significantly reduce the times-to-detection/confirmation. Because limiting the detector rate requires limiting the photon intensity, the time to detection will be approximately proportional to the width of the energy distribution and inversely proportional to the repetition rate. Treaty/dismantlement verification does not require a high photon flux per shot; hence for this application, development of high repetition rate and low energy spread are priorities over peak flux per shot. The MPS must be able to target the energy of each NRF line, which for most technologies in practice requires continuous energy tuning. The narrow energy response of MPSs restricts the potential observables, which can for example limit the sensitivity to specific isotopes, providing an information barrier. Photofission signatures were also evaluated using energy dependence of the cross section and a variable energy MPS. While individual materials can be identified in this way, it is not clear whether effects of multiple materials of unknown order can be resolved, making NRF the more desirable signature.

Stakeholders in the treaty and dismantlement communities have identified a strong interest in "fingerprinting" individual weapons by deriving an intrinsic unique identifier (I-UID). High resolution imaging tools, as used for stockpile stewardship, may provide a valuable tool for such verification involving nuclear warheads. If the focus is on resolving smaller spatial features, an MPS-based system has the potential to reduce the size of observable features by a factor of 5 or more in equal exposure time, and potentially by a factor of $\sim 200$ (likely with longer exposure time), compared to CoLOSSIS. Furthermore, the MPS does not suffer from beam hardening due to its much narrower spectrum, and scatter effects are considerably reduced because of the small beam angular divergence. An MPS-based system offers strong advantages. The approach may require management of classified information, but would provide very specific information needed for fingerprinting, and is also applicable to stockpile management. If it were already used in stockpile stewardship efforts, familiarity with the technique and experience using it on the stockpile would ease the transition to use in treaty verification. 
The potential impact of MPS-enabled techniques for treaty verification measurements could be very significant. An MPS may enable warhead confirmation, for which there is currently no known solution, as well as enabling a unique identifier for warheads and components through high-resolution radiography. In addition, a system suitable for warhead confirmation could likely be used for dismantlement confirmation, which would reduce the number of instruments required for treaty verification.

\section{Nuclear Safeguards Applications}

In nuclear safeguards, verification of the content of spent fuel dry-storage casks to reestablish inventory after loss of continuity of knowledge ( $\mathrm{CoK})$ was identified as an important need that has not been met. The IAEA is actively pursuing a method that allows re-verification of LWR spent fuel stored in dry casks. Other safeguards applications were assessed briefly for potential MPS suitability.

The thick walls of dry storage casks and the additional attenuation and scatter in the stored assemblies make radiographic measurements for the verification of the canister's content very challenging. High flux, narrow divergence MPS "pencil" beams mitigate these challenges, and transmission could be measured along specific paths through the cask. The assembly basket occupancy, and in certain cases even missing pins, may be deduced from measured chord transmission. A photon source that produces an intense, low-divergence photon beam in the 6 to $8 \mathrm{MeV}$ energy range, as well as high yield, at or above $1 \times 10^{12}$ photons/second on target (after collimation), is required to overcome the main challenge, which is low transmission through the massive cask. A pulsed source is needed; this allows the detector to be gated with the beam pulse so that only coincident photons are accepted and the radioactive background is effectively suppressed. Such a source allows a scan for verifying assembly occupancy in a few hours in a transverse scan, where the beam propagates across the assemblies. A transverse scan is required for casks which are stored vertically and which cannot be repositioned. For casks stored (or repositioned) horizontally, scans can be conducted along the long axis of the fuel bundles, which considerably reduces the complexity of determining occupancy. In this case, a MPS with mrad divergence provides sensitivity to individual fuel rods.

Other safeguards applications where brief assessment indicates potential for significant benefit include spent fuel assay, "fingerprinting" of spent nuclear fuel (SNF) assemblies, enrichment, input accountancy for pyroprocessing, and materials assay in accident scenarios. These applications could benefit from future very high performance MPSs with energy spreads on the order of $0.1 \%$ and high beam intensities on the order of $10^{11-10^{12}} \mathrm{ph} / \mathrm{s}$. Such a source could enable transmission NRF for the direct measurement of isotopic content. Detailed simulations were deferred due to limited resources available, the high required MPS performance and relatively low priority in safeguards.

Initial assessment did not identify significant capability enhancements or a high impact potential of MPS-based systems for verification of $U_{F}$ cylinder enrichment or of fresh fuel in transport containers. An MPS-based system would be capable of measurement with high confidence, but because current research may lead to simpler, possibly passive NDA techniques, this is not a priority for further assessment. This decision could be reconsidered at a later time, based on results of research on passive techniques. 


\section{Summary}

In summary, simulations show that MPSs can offer significant benefit to cargo screening, detection, warhead verification, and nuclear fuel cask scanning. They indicate that these benefits are also likely to impact emergency response and many other application areas both within and outside nonproliferation work. Full benefit is realized with an MPS having adjustable energy in the 1.5-15 MeV range; narrow (mrad) emission angle, energy spreads from approximately $10 \%$ $20 \%$ for radiography and photofission down to $<2 \%$ for NRF; and photon fluxes delivered to the target ranging from $10^{10}$ photons/second for initial capabilities to $10^{12}$ photons/second for high performance. The identified MPS requirements appear to be within the development potential of realistic MPS candidates now under development. The high-level development path motivated by the applications simulated is described in Section III of this report.

Thomson MPSs are the leading technology for fixed-facility scientific sources, but use of such sources for nonproliferation applications is currently limited by the need for high-energy linacs, scattering laser systems, and associated shielding. Each requires large fixed facilities using conventional technology. To impact nonproliferation capabilities, an integrated demonstration of a compact source concept (including mitigation of shielding) is a logical first step, targeting photon beams of $10-20 \%$ energy spread with fluxes in the $10^{7}-10^{8} \mathrm{ph} / \mathrm{shot}$ range and at controllable $\mathrm{MeV}$ energies. This source should target demonstration of the per-shot MPS properties required for radiography, Z, and photofission measurements. Subsequetly, demonstration of MPS repetition rates $\geq \mathrm{kHz}$, and hence of average flux appropriate to applications, would be a logical second step. Core laser technologies for compact laser-plasma accelerators appropriate to Thomson MPSs are being developed under the Office of Science accelerator stewardship program. Consequently, modest development specific to the scattering laser is needed to enable a high flux source. The narrow angular divergence of Thomson sources will likely require rastering of the beam across an object, a capability that must be developed once the core MPS is operational. Rapid rastering is a primary driver for the Screening and Interdiction of Shielded SNM application. Other applications considered require pointing of the source at the tens-of-seconds timescale, which is relatively straightforward. Further development would be required to enable advanced capabilities. Specifically, for both Screening and Interdiction of Shielded SNM and Detection of Hidden SNM, a key need is laser development towards repetition rates in the tens of $\mathrm{kHz}$. Exploiting NRF signatures, important to Treaty/Dismantlement Verification and to Screening and Interdiction of Shielded SNM, motivates development of percent-level or lower energy spreads in addition to repetition rates $\geq \mathrm{kHz}$.

The principal MPS candidate other than Thomson scattering is use of nuclear reactions, including ${ }^{11} \mathrm{~B}(\mathrm{~d}, \mathrm{n} \gamma)^{12} \mathrm{C}$, which produces photons both at $15.1 \mathrm{MeV}$ and in multiple lines around 2-5 $\mathrm{MeV}$ and neutrons. Applications are currently limited by accelerator and shielding size. Emission is into a $4 \pi$ angle and unpolarized. It appears likely that nuclear reaction based sources would primarily be used for screening and related applications, since single-sided detection, treaty and safeguards applications assessed prioritize very narrow divergence beams. Development of higher fluxes, or of scenarios where multiple collimators or broader collimation can be used (accounting for scattering effects), are important to take advantage of such sources. The beam can be continuous or pulsed depending on the ion accelerator used.

To demonstrate and experimentally develop MPS applications, a sequence of laboratorybased application-focused experimental campaigns can be envisioned to verify the predictions of simulations and guide development of field applications. The focus should be on configurations that take advantage of and test MPS performance characteristics, i.e., that are both strongly attenuating and have strong scattering contributions. Tests relevant to radiography and $\mathrm{Z}$ for cargo screening and stockpile applications have been started and can be conducted early in MPS 
development, since modest energy spreads are required and typically one or a few shots per location are sufficient, easing use of low repetition rate facilities. Transmission measurements appropriate for spent fuel storage casks in Safeguards have very low transmission and will be most effective on high flux $\mathrm{kHz}$ Thomson sources. Photofission rate and signal detection tests can be conducted on thinly shielded targets using low repetition rate facilities. However, to address the integrated challenges of thickly shielded targets, such as those evaluated here for Screening and Interdiction of Shielded SNM and Detection of Hidden SNM, high-flux facilities such as a kHzclass Thomson source will be important. NRF-based tests will prioritize both high repetition rate and narrow energy spread. MPS test bed facilities typically accommodate objects up to 2-3 $\mathrm{m}$ on a side, which is sufficient for realistic tests of the assessed applications. Similarly, rastering for these cases can be emulated by moving the object scanned. While beam rastering would still need to be developed, its impact could be verified in this manner. Therefore neither object size nor rastering constrains near-term tests.

MPS compactness is an important driver of utility and of the transition from laboratory to field tests, and requirements vary by application. For Screening and Interdiction of Shielded SNM, systems in use include small buildings (such as the Passport system at the Port of Boston) similar in size to current compact MPS technology test beds. Initial installation at a nuclear facility (e.g., a national lab where shipments of nuclear material are common) could be used to monitor vehicles entering and leaving and could also be used for tests with realistic objects of interest to Stockpile Stewardship, Treaty/Dismantlement Verification, and Detection of Hidden SNM. An installation similar to the Passport system at a port would also be a good pilot deployment for establishing cargo screening capabilities. Field applications for Detection of Hidden SNM, Treaty/Dismantlement Verification, and Nuclear Safeguards Dry Cask Verification likely require systems at or below the size of a small truck (also highly desirable for Screening). Development and integration of a system at this size would thus naturally follow facility-size demonstrations in the progression toward MPS field applications. Based on successful demonstration at this intermediate scale, further development to reduce size and weight to levels needed, for example, for Emergency Response would follow.

The order in which applications-focused measurement campaigns can be executed will depend on progress on each of the development tasks identified (i.e., compactness, repetition rate, energy spread, and rastering). Early measurements will use MPSs to demonstrate radiography and photofission capabilities due to their more relaxed demands on source performance compared to NRF. New signatures enabled by MPSs may also offer additional benefit, detailed evaluation of which was beyond the scope of this work. These include backscatter radiography to constrain detection volume; "few-view radiography" or computed tomography systems for clutter resolution; polarized photofission; isotope-specific energy dependence of fission cross sections; photoneutron spectroscopy; angularly resolved scattering; and fission product isomers. 


\section{Table of Contents}

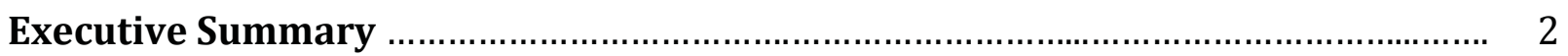

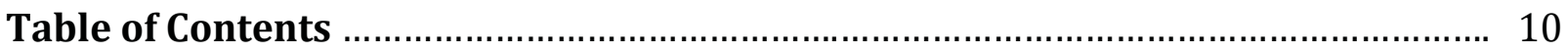

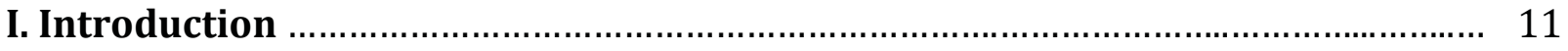

1. Methods and Signatures............................................................................. 14

2. Monoenergetic Source Examples.................................................................. 18

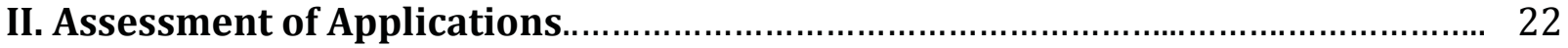

1. Cargo Screening and Interdiction of Shielded SNM .......................................... 22

2. Detection of Hidden SNM......................................................................... 56

3. Treaty/Dismantlement Verification .............................................................. 79

4. High-Resolution Imaging for Stockpile Stewardship ......................................... 97

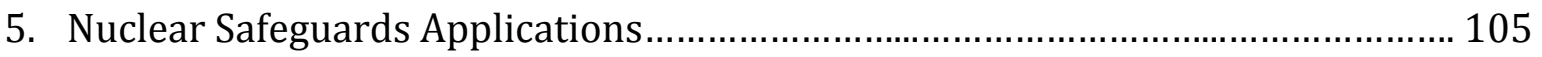

6. Emergency Response...................................................................................... 131

7. Related Applications (Waste Drum Content, Fuel Quality Control, Photonuclear Data,

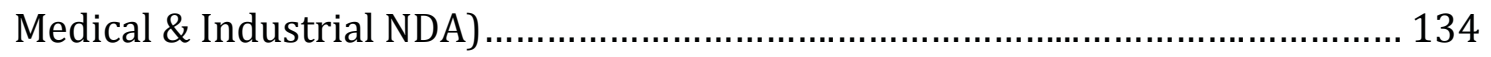

8. Comments on Nuclear Forensics Applications ………........................................ 136

III. Summary of Application Impact and MPS Development Requirements ........... 137

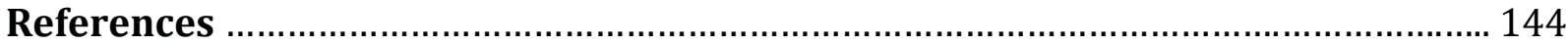




\section{Introduction}

Near-monoenergetic photon sources (MPSs) have the potential to improve sensitivity at greatly reduced dose in existing applications and to enable new capabilities in other applications, including detection of shielded SNM, treaty verification, nuclear safeguards and emergency response. MPS advantages lie in the ability to select energy, energy spread, flux, and for some MPSs also angular spread (divergence) and pulse structure, to deliver only the photons needed for the application while suppressing extraneous dose and background. Current broadband, bremsstrahlung photon sources (e.g., linacs and betatrons) deliver unnecessary dose that in some cases also interferes with the signature to be detected and/or restricts operations [2], and their beams must be collimated (reducing flux) to obtain narrow divergence. While MPSs can in principle resolve these issues, source parameters differ for various candidate technologies being developed, and additionally trade off against one another (for example, tight constraints on beam energy spread will reduce available photon intensity for MPSs based on Thomson scattering [3]). For many applications, the size and operability of an MPS and its support systems are also key restrictions. These tradeoffs, together with differences in detectors and backgrounds, represent a large operation space which must be characterized in order to understand and define needs for each application, and hence to develop and deliver a system with improved performance. This report presents a study to identify and evaluate high-impact, high-priority nonproliferation applications where MPSs can improve upon current capabilities or enable new capabilities, and to evaluate requirements to enable these advances.

Current challenging capability gaps across the nonproliferation mission space that MPSs may address were surveyed. The team reviewed existing literature and held discussions with subject matter experts from the national labs, universities and industry (Table 2) and with DNN R\&D, and with stakeholder agencies (Table 3). Discussions included a parallel DNDO-funded LANL team (Table 2) assessing MPSs for cargo screening. MPS technology developers were interviewed to assess realistic source candidates. Initial calculations were conducted and applications prioritized and selected for further study based on strong nonproliferation community interest, strong potential benefit of MPSs, and realistic concepts of operations for relatively near-term sources that are anticipated to be of truck-sized scale. Initial results were presented at several conferences, including SORMA, IEEE Nuclear Science Symposium, and Nuclear Photonics. Feedback from these events, and from follow-up discussions with cargo applications experts, were incorporated into the study. Detailed simulations and evaluations of the selected applications were then conducted. In the evaluation of the impact that monoenergetic sources would have on each prioritized application, a range of system parameters were considered, including natural and induced background radiation, beam propagation to a target, penetration, signature generation, detection sensitivity, and radiation dose to target and operators. Performance of potential monoenergetic photon sources was evaluated in the context of each application, including source tradeoffs and constraints (e.g., bandwidth, intensity, pulse structure, divergence, portability/shielding, and detector needs).

This report gives a detailed evaluation of where MPSs may have a significant impact on applications, as summarized in Table 1 of the Executive Summary. Section I.1 provides background on the methods and signatures considered, including potential novel signatures which may be accessible using MPSs. Section I.2 summarizes potential important characteristics of MPSs for general nonproliferation applications, and how these map to leading potential MPS candidates. Four nonproliferation applications prioritized for further study and detailed analysis are presented in sections II.1-II.5. Section II.1 describes Screening and Interdiction of Shielded SNM, typically in cargo containers or objects of similar size where there is access to both sides of the object. Section II.2 describes detection of hidden SNM in limited volumes, where two-sided 
access may be unavailable, addressing search of vehicles or similar objects. Section II.3 describes Treaty/Dismantlement Verification focusing on verification of nuclear weapons and their dismantlement. Techniques for unique weapon identifiers relevant to treaty verification via high resolution imaging will likely be verified first through stockpile stewardship measurements, and these are described separately in Section II.4. Section II.5 describes Nuclear Safeguards applications, focusing on verification of the content of spent fuel dry-storage casks. Simulation results were used to provide high-level MPS requirements, presented in the conclusion of each subsection. Brief evaluation of applications beyond the scope of the project and/or of lower priority is presented in the remaining subsections of section II. In addition to the aforementioned dry storage cask verification, Section II.5 covers additional safeguards applications, including spent nuclear fuel, accident scenarios, pyroprocessing, and enrichment. Section II.6 summarizes potential MPS benefits for Emergency Response. Section II.7 summarizes potential other applications to waste drum content, fuel quality control, photonuclear data, medical \& industrial NonDestructive Assessment (NDA). Section II.8 reviews nuclear forensics, where no capability enhancement was identified. Section III summarizes the requirements for the four selected applications (as described in sections II.1-II.5) and outlines the MPS technology development steps to support a coordinated program to deliver high-impact, next-generation systems with greatly reduced dose and increased detection and measurement capabilities. 
Table 2: Nonproliferation applications laboratory/university/industry discussions

\begin{tabular}{|c|c|c|}
\hline SME & Institution & Area of Expertise \\
\hline \multicolumn{3}{|c|}{ Nonproliferation Applications } \\
\hline Bob Runkle & PNNL & General, former WMS Technical Advisor \\
\hline Eric Smith & PNNL & IAEA Safeguards \\
\hline Tim White & PNNL & Radiography \\
\hline Daniel Stephens & PNNL & General, former WMS Technical Advisor \\
\hline Dave Weirup & LLNL & Emergency Response \\
\hline Richard Wheeler & LLNL & NA-22 EC POC \\
\hline Doug Wright & LLNL & Standoff Detection \\
\hline Dan Decman & LLNL & Arms Control and Treaty Verification \\
\hline Ian Hutcheon & LLNL & Nuclear Forensics \\
\hline Kiel Holliday & LLNL & Nuclear Forensics \\
\hline Harry Martz & LLNL & Cargo Security, Radiography/ Tomography \\
\hline Steve Tobin & LANL & Safeguards \\
\hline Rex Richardson & Leidos & Cargo screening \\
\hline Bill Bertozzi & Passport/MIT & Cargo screening \\
\hline Bob Ledoux & Passport & Cargo screening \\
\hline Steve Korbly & Passport & Cargo screening \\
\hline James Clayton & Varian & Cargo screening, Detection \\
\hline \multicolumn{3}{|c|}{ Other Related Applications } \\
\hline Jim Trebes & LLNL & Stockpile Stewardship \\
\hline Lou Terminello & PNNL & Stockpile Stewardship \\
\hline \multicolumn{3}{|c|}{ MPS Technology Developers* } \\
\hline Chris Barty & LLNL & Conventional linac-driven Thomson source \\
\hline Luke Campbell & PNNL & Positron annihilation source \\
\hline Igor Jovanovic & Penn. State & Nuclear reaction source \\
\hline Areg Danagoulian & MIT & Nuclear reaction source \\
\hline Richard Lanza & MIT & Nuclear reaction source \\
\hline Cameron Geddes & LBNL & Laser-plasma driven Thomson source \\
\hline \multicolumn{3}{|c|}{ DNDO-Funded MPS Requirements for Cargo Security Team } \\
\hline Sheila Melton & LANL & PI, Physicist, DNDO SNAR Test Support Team \\
\hline Cal Moss & LANL & $\begin{array}{l}\text { Physicist, DNDO SNAR Test Support Team; Nuclear } \\
\text { reaction photon sources }\end{array}$ \\
\hline Robert Estep & LANL & Physicist, DNDO SNAR Test Support Team \\
\hline
\end{tabular}


Table 3: Participants in nonproliferation applications DNN R\&D and stakeholder discussions.

\begin{tabular}{|l|c|}
\hline & Organization \\
\hline Namdoo Moon & DNDO \\
\hline Joel Rynes & DNDO \\
\hline Mark Wrobel & DNDO \\
\hline Marissa Giles & DNDO \\
\hline Jeff Musk & DTRA \\
\hline Patrick Loty & DTRA \\
\hline Hank Zhu & DTRA \\
\hline David Peterson & DTRA \\
\hline Keith McManus & DTRA \\
\hline Luke Erikson & DTRA \\
\hline Karyn Durban & NA24 - International Safeguards \\
\hline Chris Orton & NA24 -International Safeguards \\
\hline Michele Smith & Arms Control and Treaty Verification \\
\hline John Dunn & Arms Control and Treaty Verification \\
\hline Carol Mertz & Arms Control and Treaty Verification \\
\hline Elizabeth Barker & NP \\
\hline
\end{tabular}

\section{I.1 Methods and Signatures}

Important photon beam characteristics for applications may include not only photon beam energy spread, but also selectable energy, beam angular spread (divergence), emission spot size, and temporal pulse structure. Each could provide advantages (and in some cases restrictions) to performance of the principal measurement techniques used in nonproliferation photon source applications:

- Improved radiography with better contrast, Z resolution, and spatial resolution.

- Higher photo-fission sensitivity due to increased fission rate per unit dose, and reduced background.

- Enabling Nuclear Resonance Fluorescence (NRF) techniques due to high spectral density and narrow energy spread.

Bremsstrahlung-based broad energy spread photon sources are presently used, especially for radiography and photofission, but are frequently restricted in penetration and/or resolution by dose, scattering and other constraints. By eliminating low-energy photons that do not contribute to the desired signal, narrow energy spread reduces radiation dose to the object and surroundings, and also improves signal-to-noise ratios and thus sensitivity. Signal-to-background ratios may be increased by selecting the optimal photon energy for a specific application and measurement. Narrow beam divergence (the solid angle into which monoenergetic photons are emitted) may enable improved contrast by reducing scatter contributions, variation of dose across an object to ensure sufficient penetration (i.e., higher dose for pixels with higher density), and delivery of a high photon flux across a small area. Small emission spot size (the area from 
which monoenergetic photons are emitted) can improve high-resolution radiography. The time structure of the beam could be pulsed to enable prompt signatures, or near-continuous/high repetition rate to optimize use of counting measurements.

Radiography, photofission, and NRF measurements, and the photon beam characteristics important to each, are described in the following subsections. New or extended measurement techniques that could be enabled are also summarized, including polarized photofission and photoneutron spectroscopy. It is important to note that candidate MPS technologies under development may not deliver all of the desired beam characteristics. Candidate MPSs and their characteristics are reviewed in Section I.2.

\section{I.1.1 Radiography}

Radiographic techniques are used to find high-density objects within larger enclosures (e.g., cargo containers) and dual-energy radiography can further classify materials by their atomic number. Depending on the size of the enclosure, photons with energies of a few hundred keV up to $9 \mathrm{MeV}$ are used. By selecting the energy of the photon beam, MPSs have the potential to provide an image of comparable or better quality to currently-used bremsstrahlung sources, but with lower dose to the object/container [4]. Improved $\mathrm{Z}$ discrimination can be realized by selecting multiple discrete energies that maximize contrast. Because attenuation coefficients vary gradually with energy above several hundred keV, approximately 10-20\% photon energy spread is appropriate. The attenuation coefficients also serve as a useful guide to general object thickness that can in principle be penetrated. For example, as detailed in section II.1, attenuation through $45 \mathrm{~cm}$ of steel is of order $10^{-6}$ for $3 \mathrm{MeV}$ photons, which puts measurements using $<10^{8}$ incident photons [5] reasonably within reach

Beam collimation, while presenting technological challenges for rapidly scanning large objects like cargo containers, offers two important benefits. Scanning with narrow-divergence "pencil" beams greatly reduces the scattering contributions to the image and thus increases contrast as detailed in Section II.1. In addition, narrow beams can allow spatial dose adaptation. At each pixel (or resolution element), only the dose required to penetrate that pixel need be applied. This capability simultaneously ensures sufficient local penetration and overall dose minimization. As noted above, the narrow beam must then be rastered over the target of interest to construct an image. Rastering adds complexity and requires both a high photon flux incident on a small area of the object, and a pulse repetition rate fast enough to enable the desired rastering rate. On the other hand, fan beams (typical of many bremsstrahlung sources) or cone beams eliminate the need for rastering at the cost of increased scattering contributions, which degrade the image contrast and lead to higher dose requirements. The tradeoffs of these considerations, and their relationship to current methods, are considered in Section II.1, Screening and Interdiction. Where very high radiography spatial resolution is desired, the spot size from which photons are emitted becomes a limit, so a source that has smaller emission spot size has the potential to increase resolution. The use of MPSs to increase spatial resolution is discussed in Section II.4, Treaty/Dismantlement Verification \& Stockpile Stewardship.

\section{I.1.2 Photofission}

Photofission induced by high-energy photons (above $5 \mathrm{MeV}$ ) produces all of the same signatures as neutron-induced fission: prompt and delayed neutrons, and prompt and delayed gamma rays. In photofission, the key signatures that are typically leveraged are prompt and delayed neutrons and delayed gamma rays. While photofission cross-sections typically peak around $15 \mathrm{MeV}$, photoneutron production complicates the prompt neutron signature as photon energies increase, particularly above roughly $9 \mathrm{MeV}$ [6]. For bremsstrahlung sources, a challenge is that for sources with an endpoint energy below $10 \mathrm{MeV}$, only a small fraction of photons have 
energies above $5 \mathrm{MeV}$. This improves for bremsstrahlung sources at higher energies, but at energies approaching $20 \mathrm{MeV}$ photoneutron production leads to interferences with the detection of fission signatures [7]. MPSs, using moderate energy spreads of 10-50\%, can in principle deliver all photons at a selected energy that is close to the maximum photofission cross section while also keeping photon energies well below levels that are problematic for photoneutron generation. Because the photofission cross section varies with photon energy, and is different for each isotope, use of multiple MPS energies could in principle also allow differentiation of materials via use of signal ratios. Photofission signatures and methods are detailed in Section II.2, Detection of Hidden SNM, and are also discussed in Sections II.1 Cargo Screening, and II.3, Treaty/Dismantlement Verification.

\section{I.1.3 Nuclear Resonance Fluorescence}

Nuclear resonance fluorescence (NRF) provides an isotope-specific response that can be utilized for material detection and characterization. The energy of the NRF probing photon energies and responses for actinides are clustered around the 1.7 to $2.5 \mathrm{MeV}$ range $[8,9,10,11,12]$. For materials of interest for explosives detection [13] photon energies cover the range from 2 to 7 $\mathrm{MeV}$. The NRF energy range enables significant penetration through materials.

NRF measurements can be performed using either scatter or transmission techniques [13]. Scatter measurements directly detect the resonant photons emitted by the nuclei. Scatter measurements tend to be done at backward angles, greater than 90 degrees with respect to the beam, where the background is significantly lower. In transmission measurements, the photon beam is first passed through the sample and then scattered off a foil (sometimes called a monitoring foil or a witness foil), which contains the isotopes of interest. If the isotope of interest is in the sample, then the photon flux on resonance will be depleted, causing reduced signal strength as observed at the monitoring foil. Transmission measurements generally perform well when there is significant shielding or when it is desirable to measure the thickness of the material $[14,15,16]$. If the isotopic material in the sample is too thin, then transmission measurements will require very high statistics, which in practice sets a minimum thickness of material one can measure using NRF transmission techniques. As with any transmission measurement technique, if the sample is too thick, not enough beam passes through the sample to measure a significant $\mathrm{NRF}$ response off the monitoring foil. The range of suitable thickness for transmission measurements will vary from isotope to isotope, depending on the NRF response and photon attenuation of the material [15]. A bremsstrahlung experiment has shown that transmission measurements can be performed through $84 \mathrm{~g} / \mathrm{cm}^{2}$ [10] and modeling studies have shown that the NRF transmission could be applied to PWR fuel assemblies [17]. The absolute limits depend on beam and measurement parameters.

Conventional NRF measurements, either transmission or scattering using a bremsstrahlung photon beam, require spectroscopic detectors with high energy resolution. The detector of choice is generally high purity germanium (HPGe) for its large absolute efficiency and high energy resolution. The challenge with HPGe detector is the relative slow response, on the order of a few microseconds, so for bremsstrahlung photon sources one typically performs NRF measurements using a continuous-wave photon source ${ }^{1}$.

NRF measurement times are typically longer than for other signatures, and NRF is often used in combination with radiography or photofission. In detection applications, NRF may be used to confirm the presence of specific isotopes after preliminary screening using either radiography or

${ }^{1} \mathrm{~A}$ continuous wave, or $\mathrm{CW}$, source is one in which the beam is always present on the time scale of the detector. 
photofission has identified either high-Z or fissile material. NRF can be used to quantitatively measure isotopic composition. In applications such as treaty verification, this isotopic specificity may provide important advantages over radiography and photofission. Because the measurement precision is typically limited by the counting statistics, photon sources with a high spectral density are usually required.

MPSs can significantly improve NRF measurements. While the resonances are strong, they are also very narrow. The typical resonance width, after adjusting for thermal broadening, is about $1 \mathrm{eV}$, or $<1 / 1000^{\text {th }}$ of the resolution of a typical HPGe detector. The key parameter for NRF measurements is the spectral density, i.e., the number of photons per unit time and unit energy. The very narrow NRF line width prioritizes photon sources with very narrow energy spread, typically at or below $2 \%[18,19]$. By dramatically increasing the fraction of source photons that contributes to the signal, MPSs could reduce the measurement time and dose on the sample required [20]. High repetition rates are important for use of counting detectors (e.g., HPGe). The lower background of a MPS for NRF measurements may alternatively enable the use of calorimetric detectors, such as a scintillation detector covering the backward hemisphere around an isotopic scattering target [21], which could significantly improve the performance for NRF transmission measurements. Calorimetric detectors do not require single photon counting, so the pulse repetition rate of the beam is not a limitation. Use of an MPS source requires centering of the beam energy on the resonance, and separate measurements have to be performed for each isotope of interest. NRF methods are detailed in Section II.3, Treaty/Dismantlement Verification, and are also discussed in Sections II.1 Cargo Screening, and II.5, Nuclear Safeguards.

\section{I.1.4 Novel Photofission and Neutron Signatures}

In addition to the well-established signatures, new signatures have been proposed that leverage MPS characteristics and have the potential to perform discrimination among ${ }^{235} \mathrm{U},{ }^{238} \mathrm{U}$, and ${ }^{239} \mathrm{Pu}$, or to detect high explosives and contraband.

Because the dependence of the photofission cross-section on energy varies between the three isotopes, ratios of fission rates at multiple energies may allow each to be distinguished. The use of a MPS is essential for taking advantage of these differences because the ability to produce discrete energies generates stronger contrast than changing the endpoint energy of a bremsstrahlung source. This is discussed in Section II.3, Treaty/Dismantlement Verification.

Finer material discrimination may be offered by proposed methods utilizing dependence on the photon beam polarization or by measuring short-lived fission products. Using a linearly polarized $\gamma$-ray beam to induce photofission, identification of SNM based on measurement of the prompt fission neutron yields in and out of the plane of beam polarization has been investigated [22]. It was demonstrated that the fraction of prompt neutrons emitted in the polarization plane vs. out of plane differs for fissile and fissionable samples. For non-fissile samples (e.g., ${ }^{238 U}$ ), approximately 2-3 times more prompt neutrons are emitted in the polarization plane than

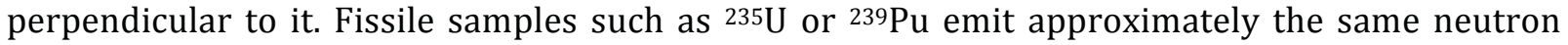
yield in both planes. Identification of fissile vs. non-fissile material was experimentally demonstrated. Further research is needed on the robustness of these signatures to shielding, configuration and fission induced by neutrons from $(\gamma, \mathrm{n})$ reactions or multiplication. A method based on detecting fission product isomers has been proposed to differentiate between ${ }^{235} \mathrm{U},{ }^{239} \mathrm{Pu}$, and the more ubiquitous ${ }^{238} \mathrm{U}$ actinides, but has not been demonstrated [23]. Using a photon source with a short (< microseconds) pulse length, it is proposed to detect $\gamma$-rays from the deexcitation of short-lived isomeric states of fission products between pulses. Assuming that two or more isomers with fission product yields that are different for ${ }^{235 \mathrm{U}},{ }^{239} \mathrm{Pu}$, and $238 \mathrm{U}$ can be identified and measured, the isotopic fraction could be determined. Short-lived isomeric states 
exist in $134 \mathrm{mTe}$ and $136 \mathrm{mXe}$ at 1691.3 and $1891.7 \mathrm{keV}$, but their half-lives of $164.1 \mathrm{~ns}$ and $2.95 \mu \mathrm{s}$, respectively, may be too short for efficient detection. Research is needed to characterize isomeric yields and resulting signatures for isomers appropriate to the pulse structure of detectors and

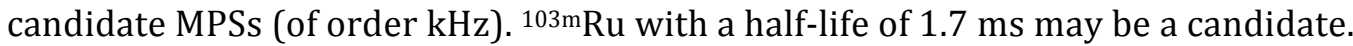

Photoneutron spectroscopy has been proposed as an explosives detection method [24], and could potentially be used to identify other contraband as well. In this technique a monoenergetic photon source is needed to produce neutrons at specific energies via $(\gamma, \mathrm{n})$ reactions. For example, a monoenergetic beam of $11.668 \mathrm{MeV}$ produces $1.035 \mathrm{MeV}$ neutrons via the ${ }^{14} \mathrm{~N}(\gamma, \mathrm{n})^{13} \mathrm{~N}$ reaction. Similarly, neutron of energies between a few hundred $\mathrm{keV}$ and a few $\mathrm{MeV}$ can be produced from other isotopes of $\mathrm{H}, \mathrm{C}, \mathrm{O}, \mathrm{Si}, \mathrm{Fe}$, and other elements so that ratios of neutron rates at specific energies could be used to identify materials [24]. Measurements appear to require a narrow energy spread $(<1 \%)$ source with selectable energies in the 4 to $12 \mathrm{MeV}$ range. Because $(\gamma, \mathrm{n})$ cross sections are typically small, producing sufficient neutron yields is challenging. A MPS, such as a Thomson source, that can deliver a high photon flux on target in very short $(<\mathrm{ns})$ pulses enabling neutron time of flight measurements for energy-resolved neutron detection, may offer a path forward. Further research is needed to assess potential of this method as well as source and detector requirements. Angularly resolved photon scattering may also be useful in characterizing materials.

Detailed evaluation of polarized photofission, prompt fission isomeric signatures, and other novel signatures is not within the scope of this report. We note that they may provide potential alternatives to NRF measurements for determining the isotopic nature of the detected material. Photofission makes less stringent demands on energy spread, but these signatures add requirements on polarization or pulse structure, respectively. As noted below, some MPSs can satisfy these requirements. Enabling use of such new signatures constitutes an added benefit to those MPSs beyond their utility for the conventional signatures that are the focus of this report.

\section{I.2. Monoenergetic Source Examples}

This report focuses on evaluation of application-dependent photon source requirements, and potential benefit. Simulations however need to include tradeoffs and constraints of candidate source technologies to provide a realistic view of what applications are accessible and of what technology gaps must be addressed in order for MPSs to meet requirements. Candidate source technologies are Thomson scattering, nuclear reactions, and positron annihilation. Discussions with source developers are noted in Table 2 and on page 5.

Thomson scattering (also referred to as inverse Compton scattering) of a laser from a counter-propagating electron beam is a well-established source of near-monoenergetic $\mathrm{MeV}$ photons for scientific users. The photon beams emitted are of narrow divergence and high intensity. Photon beam energy, yield, energy spread and polarization are continuously tunable by adjusting the electron beam and scattering laser. Correspondingly, a high quality electron beam is required for narrow photon energy spread, and photon beam intensity and angular divergence are correlated with photon energy and energy spread. Applications are currently limited by the need for high-energy linacs, in the 200-1000 MeV range, scattering lasers, and associated shielding, which are large fixed facilities using conventional technology. Current fixed facility sources include HIGS at Duke [25] and TREX/MEGA-Ray at LLNL [18]. A new generation of sources under construction includes the Extreme Light Infrastructure project in Romania [26], a source constructed for the Japan Atomic Energy Agency [19], and proposed facilities at FNAL [27], SLAC [28], the Canadian Light Source, and BNL [29]. Several projects are under way to address 
these limits and enable transportable systems by using either laser-plasma based accelerators and systems [30,31,32,33,34], or advancement of conventional accelerators $[18,35,36]$. Thomson scattering is so predictable and well understood that it is routinely used as a beam diagnostic on conventional accelerators, including the ALS linac at LBNL [37], HIGS at Duke, and Helmholtz Zentrum Dresden Rossendorf [38].

Here we consider Thomson sources at nonproliferation-relevant energies of 1-20 MeV, using potentially transportable technologies. Illustrative examples of potential performance ranges of proposed sources (not operational) are presented in Table 4.

Table 4: Examples of potential Thomson photon sources.

\begin{tabular}{|c|c|c|c|}
\hline Parameter & $2 \%$ energy spread & $10 \%$ energy spread & $\begin{array}{c}30 \% \text { energy } \\
\text { spread }\end{array}$ \\
\hline Photon energy & \multicolumn{3}{|c|}{$\begin{array}{l}\text { Continuously tunable from }<1 \mathrm{MeV} \text { to }>20 \mathrm{MeV} \\
\text { (principally restricted by electron accelerator size) }\end{array}$} \\
\hline Divergence & $\begin{array}{l}0.5 \mathrm{mrad} \text { at } 2 \mathrm{MeV} \\
0.25 \mathrm{mrad} \text { at } 8 \mathrm{MeV}\end{array}$ & $\begin{array}{l}2.5 \mathrm{mrad} \text { at } 2 \mathrm{MeV} \\
1.25 \mathrm{mrad} \text { at } 8 \mathrm{MeV}\end{array}$ & $\begin{array}{l}7 \mathrm{mrad} \text { at } 2 \mathrm{MeV} \\
3.5 \mathrm{mrad} \text { at } 8 \mathrm{MeV}\end{array}$ \\
\hline Emission spot size & $\sim$ few $\mu \mathrm{m}$ & $0.1 \mu \mathrm{m}-$ few $\mu \mathrm{m}$ & $0.1-$ few $\mu \mathrm{m}$ \\
\hline Photon yield & $\leq 10^{7} \mathrm{ph} / \mathrm{shot}$ & $\leq 10^{8} \mathrm{ph} / \mathrm{shot}$ & $\leq$ few $10^{8} \mathrm{ph} / \mathrm{shot}$ \\
\hline Polarization & \multicolumn{3}{|c|}{ selectable } \\
\hline Pulse length & \multicolumn{3}{|c|}{ microseconds to femtoseconds } \\
\hline Pulse repetition rate & \multicolumn{3}{|c|}{$100 \mathrm{~Hz}$ to $200 \mathrm{kHz}$} \\
\hline
\end{tabular}

Current Thomson sources have repetition rates at or below $100 \mathrm{~Hz}$ for conventional linear accelerators (linacs), and below $10 \mathrm{~Hz}$ for plasma based systems. However it is anticipated that future lasers will allow $1 \mathrm{kHz}$ to potentially $200 \mathrm{kHz}$ over time (note that ring or superconducting $\mathrm{RF}$ accelerators can offer much higher repetition rates, but are large systems for the required energies, and not considered here). The photon beams have narrow (mrad) divergence, which will likely require rastering of the beam across an object for many applications. Achievable intensity within this narrow divergence is high. For the quoted examples, at $\mathrm{kHz}$ repetition rate, yields could be in the range of $10^{10-10^{11}}$ photons/second. Due to the narrow (mrad) emission angle, a large fraction of the flux can be delivered to the target at moderate energy spreads in the $10-50 \%$ range. To deliver narrower energy spreads, the source must be tightly collimated (due to an energy-angle correlation in the photon emission spectrum), which reduces the flux that can be delivered. Present collimated photon energy spreads are at the few percent level for conventional accelerators, and above $10 \%$ for plasma based systems. For the longer term, sources with $0.1 \%$ energy spread, to be achieved via fine control of electron beam focusing and tight collimation, have also been proposed. Intensities in a static beam are high, with the yields cited above corresponding to a range of $10^{15}$ to $10^{17}$ photons/second/steradian. Rastering would typically reduce averaged intensity at the target plane to $10^{12}$ to $10^{16}$ photons/second/steradian by sweeping the beam across the target. While such a beam would be very intense if held stationary on one position, rastering reduces the number of photons required to extract signal from each area of the target, as detailed in section II.1, hence reducing dose required. Adapting flux as such a beam is scanned across a target can allow extraction of signal using the physics-limited minimum of dose (typically much less than used in a conventional fan beam). To accomplish rastering while preserving low energy spread, the electron beam and the collimating aperture would be scanned 
together. The electron beam could be deflected using steering magnets to accomplish rastering. For laser-plasma sources, due to the compact $(1-2 \mathrm{~m})$ size of the system, the whole accelerator and scattering system might alternatively be mounted in a turret which would rotate to steer the beam. At very high repetition rates, alternate techniques such as high-speed optical scanning mirrors [39] or magnetic steering of the laser using a preplasma [40] may be considered. Energy of the photon beam could be adapted from shot to shot either by changing the electron accelerator energy (continuous tuning) or by frequency converting the scattering laser (integer multiples of energy such as 3,6,9 MeV). Photon dose and polarization could be controlled continuously via the scattering laser. Photon emission will be in short bursts (picoseconds to femtoseconds in duration), and repetition rate must be matched to the application.

Nuclear reactions can be used to produce monoenergetic photons $[41,42,43,44]$. Typically an ion beam of relatively low energy, albeit with high current and hence often high beam power, is incident on a target to produce gamma rays of specific energies via a nuclear reaction. More than one emission line is typical. Candidate reactions include ${ }^{11} \mathrm{~B}(\mathrm{~d}, \mathrm{n} \gamma)^{12} \mathrm{C}$, which produces photons both at $15.1 \mathrm{MeV}$ and in multiple lines around 2-5 MeV and neutrons [45]. The gamma rays at $15.1 \mathrm{MeV}$ and at 4.4 MeV could be exploited for Z-discrimination based on dual energy transmission. Furthermore, the gamma rays at $15.1 \mathrm{MeV}$, as well as the neutrons produced, could be used to stimulate fission. Other candidate reactions include ${ }^{19} \mathrm{~F}(\mathrm{p}, \gamma \alpha)^{16} \mathrm{O}$, which produces gammas with energies of $\sim 6 \mathrm{MeV},{ }^{11} \mathrm{~B}(\mathrm{p}, \gamma){ }^{12} \mathrm{C}$ with energy lines at $4.4 \mathrm{MeV}(97 \%), 11.7 \mathrm{MeV}$ (97\%), and at $16 \mathrm{MeV}(3 \%)$. Gamma rays at $15.1 \mathrm{MeV}$ can also be produced by the inelastic scattering of protons on ${ }^{12} \mathrm{C}\left({ }^{12} \mathrm{C}\left(\mathrm{p}, \mathrm{p}^{\prime} \gamma\right)^{12} \mathrm{C}\right)$. The cross section is 4 times higher than that of the ${ }^{11} \mathrm{~B}(\mathrm{~d}, \mathrm{n} \gamma)^{12} \mathrm{C}$ reaction but a high proton beam energy of $\sim 20 \mathrm{MeV}$ is needed. Emission is unpolarized and into $4 \pi$. While not isotropic, variation in emission with angle is typically over very broad angles (i.e., the yield at 90 degrees is lower than that along the beam axis) [46]. Designs have been proposed for systems capable of yields in the range of $10^{8}$ to (at very high current) $10^{11}$ photons/second into $4 \pi$ (i.e. $10^{7}$ to $10^{10}$ photons/second/steradian). The usable photon fraction would depend on the application geometry. The beam would likely be collimated to produce a fan beam. While very narrow collimators are desirable to mitigate scattering issues (as discussed in Section II.1), this discards almost all of the source photon flux. To meet application needs for flux on target, and given realistic source fluxes, the collimated beam will likely need to subtend a much larger angular spread than Thomson sources. For example, a relatively broad fan collimator of 2 degrees by 30 degrees might result in only 105-108 photons/second on target. Multiple narrowly-collimated beams may be prepared relatively easily with appropriate collimator design, in which case a nuclear reaction source could in principle be used to simultaneously probe multiple objects. Such sources also can be used to produce neutrons, which can also be used to discriminate among various materials. Assessment of neutron signatures was beyond the scope of this report. The beam can be continuous or pulsed, depending on the ion accelerator used. Applications are currently limited by accelerator and shielding size, and a DNDO TAR project is in progress on these sources.

Annihilation of a relativistic positron beam, either in a solid target or with an electron beam, has also been investigated in the past as a potential photon source [47]. The $511 \mathrm{keV}$ photon produced by the annihilation is upshifted and forward peaked by the relativistic motion, resulting in a moderately low energy spread (typically order of $10 \%$ ) and moderate divergence (10s of mrad level) photon beam at few to $10 \mathrm{MeV}$ energies. Discussions with the PNNL team that led a recent DNN R\&D-sponsored work on this topic [48] did not identify such sources as being attractive compared to other source concepts currently under development, so a more detailed parameter space was not considered. 
MPS candidates can be compared to systems currently in use. Bremsstrahlung-based systems produce continuous energy spectra up to a cut-off energy given by the energy of the electron beam used to generate them. Typical energies include 3,6, and $9 \mathrm{MeV}$. The beam is forward directed with large divergence, and is typically collimated into a "fan" beam to mitigate scattering issues and ease detector setup. Typical angles are approximately 2 degrees by 30 degrees. Beam hardening filters can be used to cut off the lowest-energy photons, but also attenuate higher energy photons hence reducing usable flux. Linacs are the most commonly used accelerators, typically producing tens of microamperes of average current (delivered in pulses at $\sim 100 \mathrm{~Hz}$ ), giving photon yields of order $10^{13}$ photon/second/steradian on axis or $10^{11}$ photon/second into a fan collimator of 2 degrees by 30 degrees. Higher current (multi-mA), nearly continuous beam Rhodotrons are under development and can produce yields approximately two orders of magnitude higher than linacs but are large systems. An alternative at low energies is a ${ }^{60}$ Co source, emitting lines at 1.17 and $1.33 \mathrm{MeV}$. These energies are not as penetrating, and flux is of order $3.5 \times 10^{9} \mathrm{ph} / \mathrm{s} /$ steradian.

The assessed potential performance of both Thomson/Compton- and nuclear-reaction-based MPS candidate technologies were used as constraints on the simulation inputs. This includes both potential yield, which may constrain sensitivity as well as source tradeoffs (such as energy spread vs. yield for Thomson sources) that impact usage scenarios. MPS candidates were compared to bremsstrahlung-based systems, as used currently, that produce continuous energy spectra up to a cut-off energy given by the energy of the electron beam used to generate them. Typical energies include 3, 6, and $9 \mathrm{MeV}$. Variation of parameters was assessed to understand which parameter ranges deliver benefit for each application. Extrapolation of performance is discussed to assess performance in cases where yield beyond that of near-term MPS candidates would be desirable. This report focuses on radiography, photofission, and NRF measurements, and the photon beam characteristics important to each. New or extended measurement techniques, which could be enabled, are also in some cases identified, but are beyond the scope of this report to detail. These include backscatter radiography, polarized photofission, and photoneutron spectroscopy. 


\section{Applications Assessments}

\section{II.1. Cargo Screening and Interdiction of Shielded SNM}

\section{II.1.1. Introduction}

The potential smuggling on US soil of illicit radiological or special nuclear material (SNM) hidden in cargo containers remains a critical national security issue and a challenging technical problem. Similar challenges apply to other common cargo modes, including train cars and trucks. Screening techniques should clear vehicles accurately and must minimally affect the flow of commerce, which imposes severe constraints on scanning time and acceptable false positive rates: a positive event flags a container for inspection imposing added delays and costs. Cargo container screening, and by extension vehicle screening, for the detection of hidden SNM is generally viewed as a multiple-stage process with the goal to quickly clear a vast majority of containers while a small percentage will be flagged as suspicious and will require additional screening. Radiography is the first method to identify dense regions of the cargo. Dual-energy radiography is being developed to differentiate low-Z from high-Z materials by taking advantage of the difference in relative importance of Compton scattering and pair production processes as a function of $\mathrm{Z}$ and energy. Where a radiograph shows a region of interest within the container or vehicle with a suspicious high-Z material, it is envisioned that the container could then go though a final screening step to resolve the alarm, i.e., to unambiguously ascertain the absence of SNM. These active interrogation systems are still in the research and development stage and are not yet deployed. They rely on the characteristic signatures of fission, namely, prompt neutron and delayed gamma and neutron emissions, or on nuclear resonance fluorescence (NRF).

The large available volume of a container, relative to the small volume of a significant mass of SNM, make detection challenging. Table 5 shows significant quantities of SNM defined by the International Atomic Energy Agency (IAEA) and by the Department of Homeland Security (DHS) Domestic Nuclear Detection Office (DND0) [49, 50, 51]. Intermodal cargo containers are typically $2.4 \mathrm{~m}$ wide by $2.4 \mathrm{~m}$ high by 6 to $12 \mathrm{~m}$ ( 20 to $40 \mathrm{feet}$ ) long, with thin steel walls. The maximum load of a $40 \mathrm{ft}$ container is generally around 27 tons, although it has been noted anecdotally that loads of up to $20 \%$ over that limit are not uncommon. While the maximum theoretical density of a homogenous cargo filling the entire volume of a $40 \mathrm{ft}$ container is about $0.6 \mathrm{~g} / \mathrm{cm}^{3}$, radiographs of low-density containers commonly show a variety of cargo spatial heterogeneity [52]. In addition, when cargos are sufficiently thick or dense to limit beam transmission, radiographs show very little to no information due to a lack of signal and a lack of contrast [53]. Photon scatter in cargos has proven to be a significant contribution to the signal detected in radiographic images and can lead to degraded contrast. The ability to penetrate 20 and $40 \mathrm{~cm}$ of steel is one of the tests used to gauge the performance of a screening system. The areal density of a $40 \mathrm{~cm}$ thick steel object at full density is twice the areal density of a homogeneously filled container (which would be $240 \mathrm{~cm}$ in thickness at $0.6 \mathrm{~g} / \mathrm{cm}^{3}$ ) and could represent a conservative approximation of some of the denser objects found in cargos. $\mathrm{Z}$ discrimination tests include the ability to resolve a small high-Z object hidden behind thick steel shielding. Final screening for alarm resolution using photofission or NRF must also be evaluated in similar shielding scenarios. Additional important performance metrics include spatial resolution sufficient for the significant object of interest, scanning speed, and radiation dose. 
Table 5: IAEA and DHS standards for a significant quantity of SNM.

\begin{tabular}{|l|c|c|c|c|c|}
\hline & \multicolumn{2}{|c|}{ IAEA significant quantity } & DHS CAARS & \multicolumn{2}{|c|}{ DHS NRIP2 $^{2}$} \\
\hline Material & HEU & Pu or ${ }^{233} \mathrm{U}$ & $\mathrm{Z}>72$ & \multicolumn{2}{|c|}{ SNM } \\
\hline $\begin{array}{l}\text { Quantity } \\
\text { enrichment }>25 \%\end{array}$ & $8 \mathrm{~kg}$ & $100 \mathrm{~cm}^{3}$ cube $^{*}$ & $\begin{array}{c}\text { Target } \\
4 \mathrm{~kg}\end{array}$ & $\begin{array}{c}\text { Goal } \\
2 \mathrm{~kg}\end{array}$ \\
\hline $\begin{array}{l}\text { Sphere } \\
\text { diameter }\end{array}$ & $>13.6 \mathrm{~cm}$ & $\sim 9.2 \mathrm{~cm}$ & $\begin{array}{c}{ }^{*} \text { cube, of side } \\
\text { length } 4.6 \mathrm{~cm}\end{array}$ & $\sim 7.3 \mathrm{~cm}$ & $\sim 5.8 \mathrm{~cm}$ \\
\hline
\end{tabular}

1 CAARS: Cargo Advanced Automated Radiography System

2 NRIP: Nuclear and Radiological Imaging Platform

\section{II.1.2. Current Status}

The last twelve years have seen continued efforts to develop screening techniques to detect shielded SNM that could be hidden in intermodal cargo containers, ranging from passive detection systems to active systems relying on an external radiation source to image or probe the cargo, but finding a practical solution that is not only accurate but also fast and low dose remains a challenge. Screening techniques should minimally affect the flow of commerce, which imposes severe constraints on scanning time and acceptable false positive rates: a positive event flags a container for inspection imposing added delays and costs. Furthermore, radiation exposure must be kept to acceptable levels, due mainly to the possible presence of stowaways hidden in cargo containers. Finally, acceptance will also be impacted by the perceived risks associated with radiation at deployment locations and by potential activation of cargos. This motivates the structuring of cargo container screening, and by extension vehicle screening, for the detection of hidden SNM as a multiple-stage process with the goal of quickly clearing most containers while a small percentage will be flagged as suspicious and will require additional screening. Table 6 summarizes existing specifications that are part of two DNDO advanced technology demonstration (ATD) programs. Ideally, in the case of sea-going intermodal cargo containers, screening would occur at foreign ports of departure, but due to economic and geopolitical realities, screening usually takes place at US borders and ports of entry. At such ports, customs inspections are important, and hence a system capable both of identifying SNM and of identifying non-nuclear contraband, explosives, or undeclared items is of strong interest. These other items may be low -Z (e.g., drugs), presenting different measurement challenges. This section describes both the screening techniques currently deployed and those being developed.

Table 6: DNDO Advanced Technology Demonstration cargo scanning specifications.

\begin{tabular}{|l|c|c|c|}
\hline \multirow{2}{*}{$\begin{array}{l}\text { Material } \\
\text { Quantity }\end{array}$} & CAARS & \multicolumn{2}{|c|}{ NRIP } \\
& $100 \mathrm{~cm}^{3}$ cube & \multicolumn{2}{|c|}{ SNM } \\
\cline { 3 - 4 } & & $4 \mathrm{~kg}$ & $2 \mathrm{~kg}$ \\
\hline Scan time of 40ft container & $<1.5 \mathrm{mn}$ & $<2 \mathrm{mn}$ & $\sim 30 \mathrm{~s}$ \\
Scanning speed & $80 \mathrm{~cm} / \mathrm{s}$ & $10 \mathrm{~cm} / \mathrm{s}$ & $40 \mathrm{~cm} / \mathrm{s}$ \\
\hline Threat localization & & $<30 \mathrm{~cm}$ & $<10 \mathrm{~cm}$ \\
\hline Maximum exposure & & \multicolumn{2}{|c|}{$<500 \mathrm{mrem}$} \\
\hline
\end{tabular}

Passive detection systems, deployed at ports of entry and border crossings, are used to screen 99.98\% of containers. Trucks and vehicles drive through static radiation portal monitors (RPMs) combining two types of detectors: helium tubes surrounded by a moderator for neutron detection, and plastic or NaI detectors for gamma detection. However, it has been recognized that the false alarm rates due to cargos containing sufficient quantities of Normally Occurring Radioactive 
Materials (NORM), and the inability to detect characteristic emissions from shielded SNM, are limiting factors for passive detection systems. This motivates development of active approaches.

Selected containers are flagged as suspicious based on intelligence, shipper information and cargo manifests, and these can be imaged with portable/movable or static photon-source based radiographic systems. Software enhanced images are reviewed by customs agents who then decide if a container should be opened and inspected or if it should be cleared. Practically, these systems are used to find contraband and explosives rather than SNM, and, to our knowledge, there are currently no deployed active interrogation systems specifically designed to uniquely identify SNM signatures. Commercial cargo screening radiographic systems rely on a ${ }^{60}$ Co source or on linac-based bremsstrahlung sources with multi-MeV end-point energies. These are integrated in both static and mobile systems. All these systems perform horizontal scans using a source (having initially broad divergence) collimated to obtain a vertical fan beam covering the height of the container. Aligned with the source and on the opposite side of the container, the detection system generally consists of one or several vertical linear arrays of detectors that image the full height of the container at once $[54,55,56,57]$. To scan the horizontal length of the container, either the source/detector or the container is moved.

The energy distribution of the photon source in a radiographic system defines transmission through cargo, which is one important determinant of the dose required to form an image. For ${ }^{60} \mathrm{Co}$ sources, which emit near $1.25 \mathrm{MeV}$, transmission through $30 \mathrm{~cm}$ of steel is order $3.6 \times 10^{-6}$. This is 57 times lower than for $3 \mathrm{MeV}$ photons. Bremsstrahlung sources produce very broad photon spectra. Typical sources operating at 6 and $9 \mathrm{MeV}$ without beam hardening filters have average energies of $\sim 1.23 \mathrm{MeV}$ and $1.73 \mathrm{MeV}$, but this is not representative of transmission. Photons with energies greater than $2 \mathrm{MeV}$ account for $\sim 20 \%$ and $30 \%$ of the spectrum, respectively, but account for over $95 \%$ of all transmitted photons through $30 \mathrm{~cm}$ of steel. Hence, transmissions for 6 and $9 \mathrm{MeV}$ un-hardened bremsstrahlung sources are $\sim 5.3 \times 10^{-5}$ and $1.2 \times 10^{-4}$ respectively. Some commercial bremsstrahlung systems at 6 and $9 \mathrm{MeV}$ are built with beam hardening filters and report beam average energies around 2 and $3 \mathrm{MeV}$ respectively, which improves transmission. For a $9 \mathrm{MeV}$ bremsstrahlung beam hardened with a $5 \mathrm{~cm} \mathrm{~Pb}$ filter, the transmission increases by a factor of $\sim 2.3$, to $2.7 \times 10^{-4}$. Significant low energy photon flux remains. Due to this low energy flux, the transmission of the hardened bremsstrahlung beam is still $3.4 \times$ lower than that for a $9 \mathrm{MeV}$ MPS, which would have a transmission of $9.24 \times 10^{-4}$.

The naturally broad energy spread of a bremsstrahlung source presents several issues. Low energy photons contribute to the overall dose, but their transmission through cargo is very low and hence they make nearly no contribution to the detected signal. Beam intensity has to be increased to obtain a sufficient flux of higher energy photons that can penetrate thick cargos, further increasing the dose. Finally, photon scatter in cargos has proven to be a significant contribution to the signal detected in radiographic images and can lead to degraded contrast, a contribution that cannot be easily deconvolved from the transmitted beam. To reduce scatter contributions, existing systems' configuration consists of a vertical fan beam and a linear array of detectors, usually made of $\mathrm{CdWO}_{4}$.

While use of a narrowly collimated "pencil" beam scanned across the target is known to further reduce scattering contributions, this is not done routinely for two reasons. Narrow collimation discards most of the source flux, requiring significant increases in linac current, and requires a scanning system to raster the beam across the target. Both add complexity and can increase scan time. Some systems are built with linear arrays of Cherenkov detectors that have an energy threshold of $\sim 1 \mathrm{MeV}$. Since scattering is most severe for low energy photons, this can reduce the dose required to generate an image with similar contrast. Miller et al. conducted experimental and simulation studies to identify and quantify the main sources of scatter for a 
VACIS radiographic system with a collimated fan beam 3.2 degrees wide and a vertical opening of 33 degrees. The main sources of scatter were the container's content itself, various supporting structures in the system, and the truck bed underneath the container. For a given cargo, increases in density resulted in large increases in the scatter contribution, to levels several times larger than the transmitted signal [53].

Dual-energy radiography can be used to differentiate low $\mathrm{Z}$ from high $\mathrm{Z}$ materials by taking advantage of the difference in the relative importance of Compton scattering and pair production processes as a function of $\mathrm{Z}$ and energy $[58,59]$. Compton scattering is the dominant mode of interaction between 1 and $9 \mathrm{MeV}$ for low $\mathrm{Z}$ materials, while high $\mathrm{Z}$ materials pair production cross-sections become significant above $5 \mathrm{MeV}$. Systems were developed for SNM detection that relied on bremsstrahlung fan beams, with 6 and $9 \mathrm{MeV}$ end-point energies for example. The goal is to isolate high $\mathrm{Z}$ objects-where $\mathrm{Z}>72$ is often defined as "high" -in radiographs of cargos. This has remained a challenging problem so far. Current systems have to contend with convolution of two broad bremsstrahlung spectra and with scattering due to the fan beam collimation. All tested systems in the CAARS ATD program had high false alarm rates and/or inconsistent automatic detection of high-Z objects, possibly caused by scatter in some cases [60].

"Few view radiography" and computed tomography systems have been proposed. These have the potential to improve identification of materials, especially in complex cargos. However they are not currently being pursued due to increased system complexity and longer scanning time.

A Passport scanning system (Passport Systems Inc.) is under development based on their EZ3D method to determine effective Zs in a voxelized container. To do this, a $3.5 \times 3.75 \mathrm{~cm}$ bremsstrahlung square beam is generated at the top of the container by a high-current $9 \mathrm{MeV}$ Rhodotron accelerator. The beam successively irradiates vertical sections of the container through 64 ports. Photons are detected at about a 110-degree angle with respect to the beam by two vertical arrays of $2 \times 30$ collimated NaI detectors, one on each side of the container. The 3D position in the container or voxel is defined by the intersection of the beam and the collimated field of view of a detector. The effective $\mathrm{Z}$ of each voxel is determined from the detected energy spectrum of backscattered X-rays below and above $511 \mathrm{keV}$. The presence of high-Z material in a voxel results in an increase in secondary bremsstrahlung photons and is determined from the ratio of high energy to low energy events in the recorded pulse height spectrum [13]. The Rhodotron can deliver two additional beam energies of 3 and $6 \mathrm{MeV}$. The Rhodotron's high current (relative to linacs) of up to $3 \mathrm{~mA}$ is important to produce the narrowly collimated beams used, while maintaining sufficient photon flux. The dose delivered to the cargo during scanning for $\mathrm{Z}$ determination was estimated to be $\sim 400 \mathrm{mrad} / \mathrm{scan}$. It has also been recommended that the size of the Rhodotron be reduced.

Where a radiograph shows a region of interest within the container or vehicle with a suspicious high-Z material, it has been envisioned that the container could then go though a final screening step to resolve the alarm, i.e., to unambiguously ascertain the absence of SNM. These active interrogation systems are still in the research and development stage and are not deployed. They rely on the characteristic signatures of fission (namely, prompt and delayed gamma and neutron emissions following fission) and/or on nuclear resonance fluorescence (NRF). Detection systems for prompt and delayed neutron and gamma emissions using linac-based photofission have been under development. Passport Systems Inc. has developed a cargo screening system that combines high energy backscatter (EZ-3D) imaging, prompt neutron photofission, and NRF [61]. Once a high-Z region of interest (ROI) is identified, it is selectively irradiated and NRF lines are measured with a detection system on one side of the container, consisting of 9 HPGe detectors mounted on a tower that can be moved in the plane of the beam to view the ROI [62]. In the Technology Demonstration \& Characterization testing of the Passport system conducted by DNDO, 
the NRF component has not been successful in SNM detection in tests with HEU and DU targets in spite of long measurement times of about 10-15 minutes and large radiation doses to the cargo in the 10s of krad range [62]. The NRF technique has been more successful in the measurement of low-Z isotopes for the detection of explosives and contraband.

In addition to photon-based systems, a muon tomography approach and several neutron activation systems are being developed. Muon tomography (Decision Sciences Corp.) relies on muons - a naturally occurring radiation source that can penetrate thick cargos - to generate a Zbased 3D map of the cargo material content. It is a passive detection technique that does not deliver an additional radiation dose. While the multi-mode passive detection systems (MMPDS) website quotes scanning times of 45 seconds for a $40 \mathrm{ft}$ container, the system is unlikely to uniquely identify SNM within that time [63][50]. Neutron-based systems are also candidates. The detection of beta-delayed gamma rays emitted following fission in HEU was demonstrated using a system based on a $7 \mathrm{MeV}$ neutron source and large plastic detectors [64]. Rapiscan systems relied on differential die-away (DDA) of the scattered neutron signal and on differential time of flight (DTOF) depending on the thickness of the cargo surrounding the SNM [65] [66]. It is unclear that these systems will be deployed due to the known issues associated with neutron active interrogation techniques, such as high neutron dose to stowaways, cargo activation, or the impact of shielding requirements on a system footprint. The assessment reported here focuses on photon source-induced signatures.

\section{II.1.3. Potential Capabilities and Advantages of MPS-based System}

Several MPS characteristics could lead to important capability improvements in radiography applications. Desirable characteristics include the ability to select the optimal beam energy to maximize transmission and to minimize dose, to tightly control the energy spread, and to take radiographs at more than one energy for $\mathrm{Z}$ determination. MPSs with small beam divergence allow "pencil beam" scanning for a drastic reduction of the scatter contribution to the image, and (if the photon beam flux can be adjusted) dose adaptation as a function of cargo spatial distribution. Not all MPSs will have all of these capabilities.

The main characteristics of potential MPSs and other MeV-range photon sources relevant to cargo screening are summarized in the Introduction, section I.2. ${ }^{60}$ Co sources and linacs are parts of deployed radiographic systems used to detect contraband and explosives in containers. Rhodotrons (and linacs) are part of prototype systems that were tested during DNDO CAARS and SNARE ATD with the goal to detect high Z material, to ascertain the presence of SNM with photofission and to identify fissile isotopes with NRF. Finally, MPSs based on nuclear reactions and Thomson scattering are in the developmental stage.

Following an initial assessment of the potential benefits of MPS characteristics on general radiographic imaging [1] higher-fidelity simulations have been conducted to evaluate radiography, as well as photofission and NRF. These detailed assessments quantify how use of MPSs can result in higher transmission and reduced scatter, leading to higher contrast and lower radiation dose as well as improved $\mathrm{Z}$ discrimination. Each of these benefits is discussed in the following sections. The first subsection describes the photon beams used in subsequent simulations. For radiography, the ability to select the optimal beam energy that can maximize transmission and minimize dose is next evaluated without scattering, indicating MPS advantages of three- to four-fold due to energy spectrum alone. Simulations are then described which evaluate the effects of scattering on image quality, showing that the greatest benefit is obtained by photon sources with narrow-divergence (pencil) beams which are scanned across the target. Improved Z-discrimination is next assessed by evaluating use of ratios of discrete MPS energies 
versus continuous bremsstrahlung distributions. Scattering contributions to Z-discrimination resolution are included. Use of small beam divergence MPSs to allow pencil beam scanning is found to allow a reduction of the scatter contribution to image degradation, and for 2D dose adaptation as a function of cargo spatial distribution. Lastly, secondary screening is evaluated where MPSs can generate stronger photofission signatures and enable more sensitive NRF measurements.

\section{II.1.4. Assessment of MPS Performance: Simulations}

\section{II.1.4.1. Radiography}

Potential radiography capability improvements for cargo screening from use of MPSs were evaluated in comparison to bremsstrahlung beams. Simulations were performed to estimate benefits in transmission, dose reduction and $\mathrm{Z}$ discrimination due to the advantageous energy spectrum of an MPS. Simulations included the impact of the scatter contribution to the image and potential mitigation via control of both the photon beam energy spectrum and angular spread. Angular spread is important to evaluate the difference between the fan beams produced by most bremsstrahlung sources and MPSs, which may produce fan, cone or narrow-divergence pencil beams. The latter would be scanned across a target to form an image.

\section{Simulation Input: Bremsstrahlung Beams}

The energy distribution of the photon source in a radiographic system defines transmission through cargo, which is one important determinant of the dose required to form an image. Some commercial bremsstrahlung systems at 6 and $9 \mathrm{MeV}$ are built with beam hardening filters and report beam average energies around 2 and $3 \mathrm{MeV}$ respectively, which improve transmission. To allow comparison with MPS candidates, bremsstrahlung beams were modeled for use in the simulation study.

Energy spectra were first calculated for unhardened 6 and $9 \mathrm{MeV}$ bremsstrahlung beams as a function of angle in 1 degree increments and then transmitted through representative hardening filters of $0.5 \mathrm{~cm}$ of $\mathrm{Cu}$ and $1.0 \mathrm{~cm}$ of W, respectively using the NIST XCOM data [67]. The resulting spectra are shown in Figure 1. The average energy of the attenuated on-axis beams and overall transmission compared to the unhardened beams are summarized in Table 7 . While the W filter reduces the contribution from the low energy photons as shown by the increase in average energy of the beam, the intensity is also significantly reduced to $30 \%$ of the unhardened beam. The $\mathrm{W}$ hardened spectrum is used in the simulations presented here because of its improved transmission due to the higher mean energy. This represents a best-case bremsstrahlung source. It was compared to MPS beams of various energy spreads to assess potential MPS benefits to applications.

Table 7: Simulated 6 and $9 \mathrm{MeV}$ bremsstrahlung beams on-axis.

\begin{tabular}{|c|c|c|c|c|c|c|}
\hline Energy endpoint & $6 \mathrm{MeV}$ & & & $9 \mathrm{MeV}$ & & \\
\hline Filter & Unhardened & $\begin{array}{c}0.5 \mathrm{~cm} \\
\mathrm{Cu}\end{array}$ & $1 \mathrm{~cm} \mathrm{~W}$ & Unhardened & $\begin{array}{c}0.5 \mathrm{~cm} \\
\mathrm{Cu}\end{array}$ & $1 \mathrm{~cm} \mathrm{~W}$ \\
\hline $\begin{array}{c}\text { Average Energy } \\
{[\mathrm{MeV}]}\end{array}$ & 1.29 & 1.41 & 1.83 & 1.73 & 1.89 & 2.37 \\
\hline $\begin{array}{c}\text { Transmission } \\
\text { through filter } \\
\text { compared to } \\
\text { unflattened beam }\end{array}$ & - & $76.4 \%$ & $28 \%$ & - & $78 \%$ & $31 \%$ \\
\hline
\end{tabular}



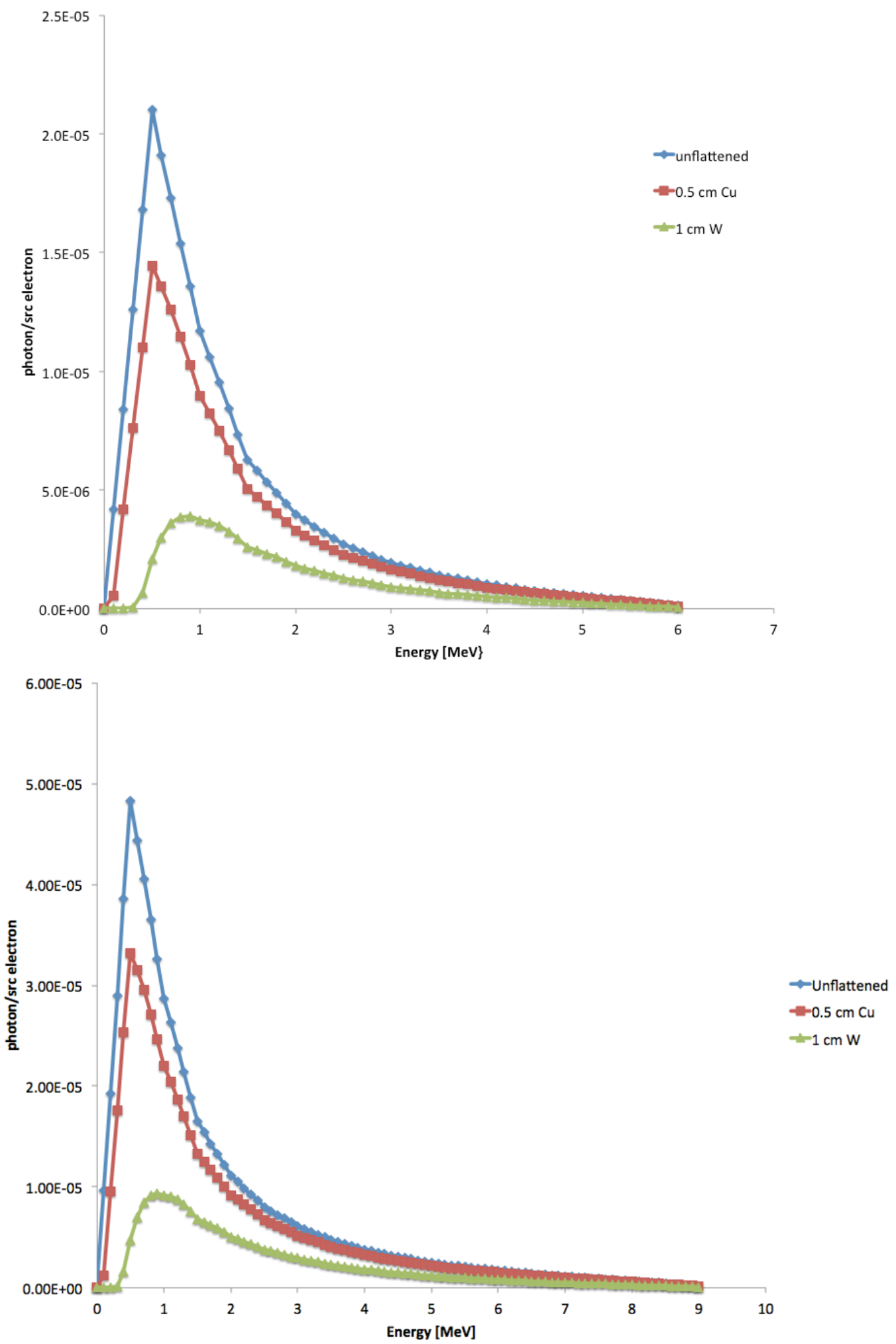

Figure 1. Simulated $6 \mathrm{MeV}$ (top) and $9 \mathrm{MeV}$ (bottom) bremsstrahlung spectra, unhardened (blue), with $0.5 \mathrm{~cm}$ Copper hardening filter (red), and with $1 \mathrm{~cm}$ W hardening filter (green). 


\section{Transmission and Dose Advantages Due To Energy Spectrum}

Using a MPS can result in a significant gain in transmission compared to a bremsstrahlung source, which could result in shorter irradiation times, lower doses or improved statistics. Analytic estimates indicated [1] that the dose reduction due to source energy spread alone, which convolves both transmission and dose per photon, could be of the order of a factor of two, but evaluation of realistic screening cases was required to generate specific dose estimations. MCNP simulations were performed to calculate transmission for MPS and bremsstrahlung beams at several energies through $20 \mathrm{~cm}$ and $40 \mathrm{~cm}$ of steel and to evaluate doses to the cargo and to a potential stowaway per source photon. The transmission results indicate the dose impact due to energy spectrum alone. For a full evaluation of the MPS impact on dose, these results must be convolved with results of simulations determining the impact of source angular spread (narrow angular spread sources can reduce scattering impact, reducing the number of photons required to form an image, and hence dose), as discussed in following sections. The benefits of dose modulation across the cargo enabled by scanning with a narrow divergence MPS beam must be considered.

A two-step approach was taken to compare different beam types in terms of dose to a stowaway. A first set of simulations calculated the transmission as the dose per source photon deposited in a $1 \times 1 \mathrm{~cm} \mathrm{CdWO}$ detector either 3 or $10 \mathrm{~cm}$ long by a pencil beam penetrating 20 $\mathrm{cm}$ or $40 \mathrm{~cm}$ thick steel centered in a cargo container. The dimensions of the container are $1.2 \mathrm{~m} \times$ $2.4 \mathrm{~m} \times 2.4 \mathrm{~m}$. The detector face is located $80 \mathrm{~cm}$ from the exit wall of the container, or $200 \mathrm{~cm}$ from the center of the container. The detector is sandwiched between tungsten collimator leaves extending $30 \mathrm{~cm}$ from the face of the detector. This configuration shields the detector from lateral secondary scatter contribution.

In a second step, the body dose is simulated with a model that includes a vertical water cylinder $180 \mathrm{~cm}$ high and $40 \mathrm{~cm}$ in diameter inside a cargo container to emulate a human body, next to the wall facing the source (Figure 2). The photon source is a surface source with a cross section identical to the body dimensions, i.e., $40 \mathrm{~cm}$ wide by $180 \mathrm{~cm}$ high, and photons are emitted normal to the source plane and perpendicular to the cylinder axis. Bremsstrahlung beams were hardened with $0.5 \mathrm{~cm}$ of $\mathrm{Cu}$ or $1 \mathrm{~cm}$ of $\mathrm{W}$ as described above. Results are summarized in Table 8. As expected, the dose per source photon is higher for an MPS at a given energy than for a bremsstrahlung source with that endpoint energy. This is because the MPS delivers all of its photons at the target energy, while most of the bremsstrahlung photons are at lower energies.

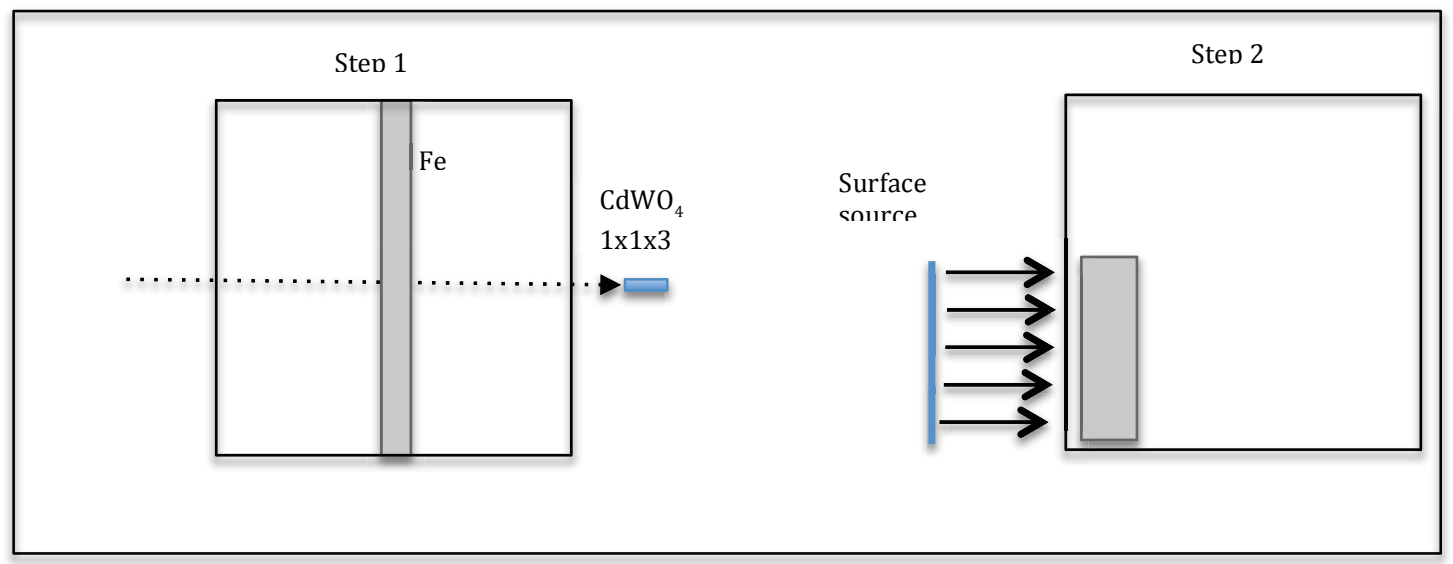

Figure 2. Models for the detector dose simulations (left) and body dose (right). 
Understanding the dose required to form a radiograph requires convolving the dose effect with the transmission data obtained in the first set of simulations.

Table 8: Simulated doses per source photon in the water cylinder for hardened bremsstrahlung beams and for MPS beams.

\begin{tabular}{|l|c|c|l|c|c|}
\hline Energy [MeV] & \multicolumn{2}{|l|}{$\begin{array}{l}\text { Bremsstrahlung } \\
\text { [Gy/source photon] }\end{array}$} & $\begin{array}{l}\text { MPS } \\
\text { [Gy/source photon] }\end{array}$ & $\begin{array}{l}\text { Dose Ratio } \\
\text { MPS/Brem }\end{array}$ & $\begin{array}{l}\text { Dose Ratio } \\
\text { MPS/Brem }\end{array}$ \\
\hline Brems. filter & $1 \mathrm{~cm} \mathrm{~W}$ & $0.5 \mathrm{~cm} \mathrm{Cu}$ & & $1 \mathrm{~cm} \mathrm{~W}$ & $0.5 \mathrm{~cm} \mathrm{Cu}$ \\
\hline $3 \mathrm{MeV}$ & - & - & $1.01 \mathrm{e}-15$ & & \\
\hline $6 \mathrm{MeV}$ & $6.33 \mathrm{e}-16$ & $4.90 \mathrm{e}-16$ & $1.75 \mathrm{e}-15$ & 2.76 & 3.57 \\
\hline $9 \mathrm{MeV}$ & $7.83 \mathrm{e}-16$ & $6.27 \mathrm{e}-16$ & $2.42 \mathrm{e}-15$ & 3.09 & 3.86 \\
\hline
\end{tabular}

For each beam type and energy, the body dose was normalized to the corresponding detector dose to obtain the dose comparison for a radiograph. In this comparison the beams differ only in their energy spectrum. The results are given in Table 9. Note that simulations for a $10-\mathrm{cm}$ long detector resulted in detector doses smaller by a factor of $\sim 2$ for all cases; thus the final dose ratios were essentially unchanged by the detector type. This is as expected since the detector length essentially sets efficiency. For this reason, only one set of dose ratios is reported. Column six (far right) reports the ratio of dose imparted by a bremsstrahlung source to that imparted by a MPS, under conditions where both deliver equal dose to the detector (transmission). This measures the part of the dose advantage of an MPS over a bremsstrahlung beam that is due to difference in energy spectrum alone.

Table 9: Ratios of expected water phantom doses for MPS and bremsstrahlung spectra for same detector signal after penetrating $20 \mathrm{~cm}$ and $40 \mathrm{~cm}$ thick steel.

\begin{tabular}{|c|c|c|c|c|c|}
\hline $\begin{array}{c}\text { Steel } \\
\text { thickness } \\
{[\mathrm{cm}]} \\
\end{array}$ & $\begin{array}{c}\text { Energy } \\
{[\mathrm{MeV}]}\end{array}$ & $\begin{array}{l}\text { Beam } \\
\text { type }\end{array}$ & $\begin{array}{c}\text { Detector } \\
\text { dose/src-photon } \\
{[\mathrm{Gy}]}\end{array}$ & $\begin{array}{c}\text { Water phantom } \\
\text { dose/src-photon } \\
{[\mathrm{Gy}]}\end{array}$ & $\begin{array}{c}\text { Dose ratio } \\
\text { Brem/MPS }\end{array}$ \\
\hline \multirow[t]{4}{*}{20} & 3 & MPS & $2.6 \mathrm{e}-14$ & $1.0 \mathrm{e}-15$ & \multirow{2}{*}{1.3} \\
\hline & 6 & Brem & $1.3 e-14$ & $6.3 e-16$ & \\
\hline & 9 & MPS & $2.7 \mathrm{e}-13$ & $2.4 \mathrm{e}-15$ & \multirow{2}{*}{3} \\
\hline & 9 & Brem & $2.9 \mathrm{e}-14$ & $7.8 \mathrm{e}-16$ & \\
\hline \multirow[t]{4}{*}{40} & 3 & MPS & $9.5 e-17$ & $1.0 \mathrm{e}-15$ & \multirow{2}{*}{0.84} \\
\hline & 6 & Brem & $7.1 \mathrm{e}-17$ & $6.3 e-16$ & \\
\hline & 9 & MPS & $2.6 \mathrm{e}-15$ & $2.4 \mathrm{e}-15$ & \multirow[b]{2}{*}{4} \\
\hline & 9 & Brem & $2.1 \mathrm{e}-16$ & $7.8 \mathrm{e}-16$ & \\
\hline
\end{tabular}

The photon energy deposited in the detector per source photon, given in column 4 in Table 9, is $\sim 10 \times$ higher for the $9 \mathrm{MeV}$ MPS beam compared to the $9 \mathrm{MeV}$ bremsstrahlung beam with $1 \mathrm{~cm}$ W filter which could mean 10 times fewer MPS photons are needed to generate an image. However, this does not translate into a reduction of the dose to the container by the same factor since the dose per incident photon for $9 \mathrm{MeV}$ MPS is $\sim 3$ times that of a $9 \mathrm{MeV}$ bremsstrahlung beam. To penetrate a $20 \mathrm{~cm}$ thick steel cargo, the dose for a $9 \mathrm{MeV}$ MPS is lower by a factor of $\sim 3$. This dose ratio increases to a factor of $\sim 4$ for $40 \mathrm{~cm}$ thickness. When the $9 \mathrm{MeV}$ MPS is compared to a softer bremsstrahlung beam filtered with $0.5 \mathrm{~cm}$ of $\mathrm{Cu}$, the $9 \mathrm{MeV}$ MPS doses are similarly lower, by factors of 3.06 and 4.14 respectively. Note that the transmission of a $3 \mathrm{MeV}$ MPS beam is only moderately higher than that of a $6 \mathrm{MeV}$ bremsstrahlung beam, resulting in similar doses to cargo. Practically, applications for a $3 \mathrm{MeV}$ beam are likely limited to small cargos or as part of a 
dual energy radiography system with few views. The simulation results evaluate transmission for realistic cases relevant to screening, and indicate that in the absence of scattering contributions, the dose reduction due to MPS energy spectrum alone is in the range of $3-4 \times$ for the most relevant energies $(\sim 9 \mathrm{MeV})$. Estimation of the total dose reduction requires convolving this result with simulations including scattering using the beam emission angle geometry of a particular MPS.

\section{Scatter Reduction, Contrast Enhancement, and Dose Reductions}

Scattering is a significant contributor to image quality degradation in existing cargo radiography systems, and simulations were conducted to evaluate the impact of MPSs in reducing these contributions. Incoherent scattering is the main photon interaction in the energy range considered here for most materials found in cargos, essentially increasing background in each pixel and lowering contrast. To first order, the cargo scatter contribution reaching the image plane depends on the energy and size of the beam at the object and is generally controlled by collimation.

Scattering contributions can be affected by energy distribution, detector configuration, and by beam emission angle (e.g., fan, pencil, or cone). Qualitatively, lower energy photons scatter more severely, so an MPS may have an advantage by delivering all of its photons near a target energy. Detector collimation can reduce the number of scattered photons that affect the image. A small beam emission angle means a small beam diameter at the target, which enables pencil beam scanning. Such scanning potentially enables a large reduction in the image contrast degradation due to scattering. The divergence requirement is set by the desired scanning resolution, since beam size at the container wall is defined by the beam divergence and the source-to-cargo distance. For container radiography applications, a beam size of $1 \mathrm{~cm}$ at the container wall can be achieved at a distance of $5 \mathrm{~m}$ with a beam divergence of $2 \mathrm{mrad}$, or at $2.5 \mathrm{~m}$ using $4 \mathrm{mrad}$. Using bremsstrahlung or nuclear reaction sources, such collimator angles would discard almost all of the available photons and would require high current. Thomson sources, on the other hand, intrinsically generate beams with few-mrad divergence. Simulations evaluated each of these effects.

Simulations first modeled propagation of a bremsstrahlung and a monoenergetic pencil beam through a steel plate to investigate scatter contribution and beam intensity profile as functions of distance from the plate. Then, simulations were conducted using both MPS and bremsstrahlung spectra of radiographic imaging including the effects of scattering to assess contrast for various beam emission angle geometries, detector collimations, and target types.

\section{Propagation of bremsstrahlung and monoenergetic beams}

To visualize the effect of scattering on the photon beam and the effect of detector stand-off distance, propagation of a $9 \mathrm{MeV}$ pencil beam (i.e., a monodirectional beam with no spatial extent) was simulated using the MCNPX code through $1 \mathrm{~m}^{2}$ steel plates, 20 and $40 \mathrm{~cm}$ thick. Both a $9 \mathrm{MeV}$ monoenergetic beam and a bremsstrahlung spectrum with $9 \mathrm{MeV}$ endpoint energy were simulated in this configuration. The flux is calculated with a mesh tally in MCNPX, using voxels 1 $\mathrm{cm}$ on a side in the plane of the beam. Figure 3 shows the photon flux map for the $9 \mathrm{MeV}$ MPS propagating through $40 \mathrm{~cm}$ of steel. The beam is heavily attenuated by the steel, and photons are scattered out of the beam into the steel. Lineouts are shown in Figure 4 for the $20 \mathrm{~cm}$ case and in Figure 5 for the $40 \mathrm{~cm}$ case. In each figure, the plot corresponding to the downstream edge of the steel (located at $\mathrm{d}=0 \mathrm{~cm}$ in the fluence map) shows a significant portion of the photons in the beam are scattered out of the beamline. At modest distances after the steel, indicated by the dashed line at $100 \mathrm{~cm}$ the unscattered beam is still very tightly focused while the scattered flux is low since the scattered photons have a wide angular distribution. At $100 \mathrm{~cm}$ from the surface of the steel, the scattered intensity is typically three orders of magnitude below the transmitted 
beam intensity. This indicates that for a narrow divergence beam, scattering contributions to image contrast degradation will be very small. The sum of the scattered photons observed on a linear detector array covering $\pm 50 \mathrm{~cm}$ from the central beam location is about $6 \%$ of the transmitted beam flux for the $40 \mathrm{~cm}$ steel plate, and near $2.5 \%$ for the $20 \mathrm{~cm}$ steel plate. Similar simulations were conducted for bremsstrahlung sources with $9 \mathrm{MeV}$ endpoint energy. These showed modestly larger scattering, with total scattered photons observed on a linear detector array covering $\pm 50 \mathrm{~cm}$ from the central beam location being about $7 \%$ of the transmitted beam flux for the $40 \mathrm{~cm}$ steel plate, and near $3 \%$ for the $20 \mathrm{~cm}$ steel plate.

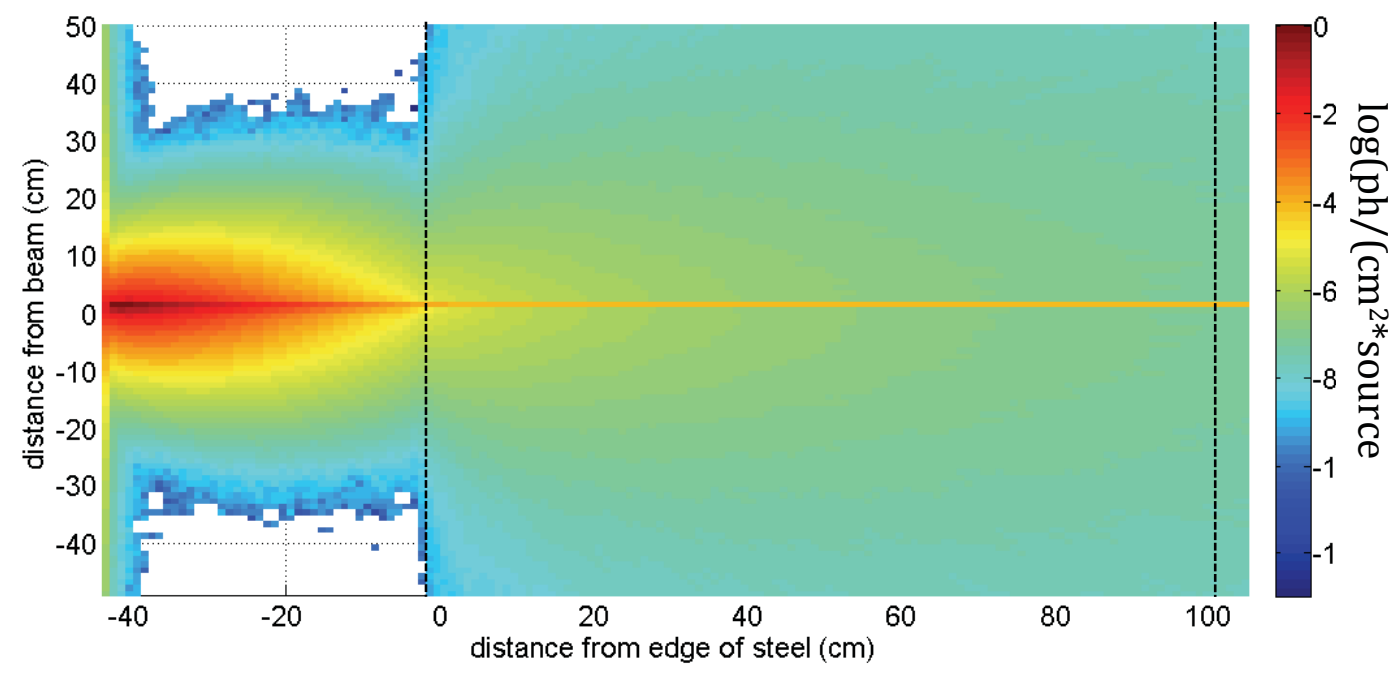

Figure 3. 2D Flux map in a plane through the beam axis for a $9 \mathrm{MeV}$ photon beam penetrating $40 \mathrm{~cm}$ of steel, dashed lines at locations of voxel rows used for flux measurements in Figures 4 and 5.

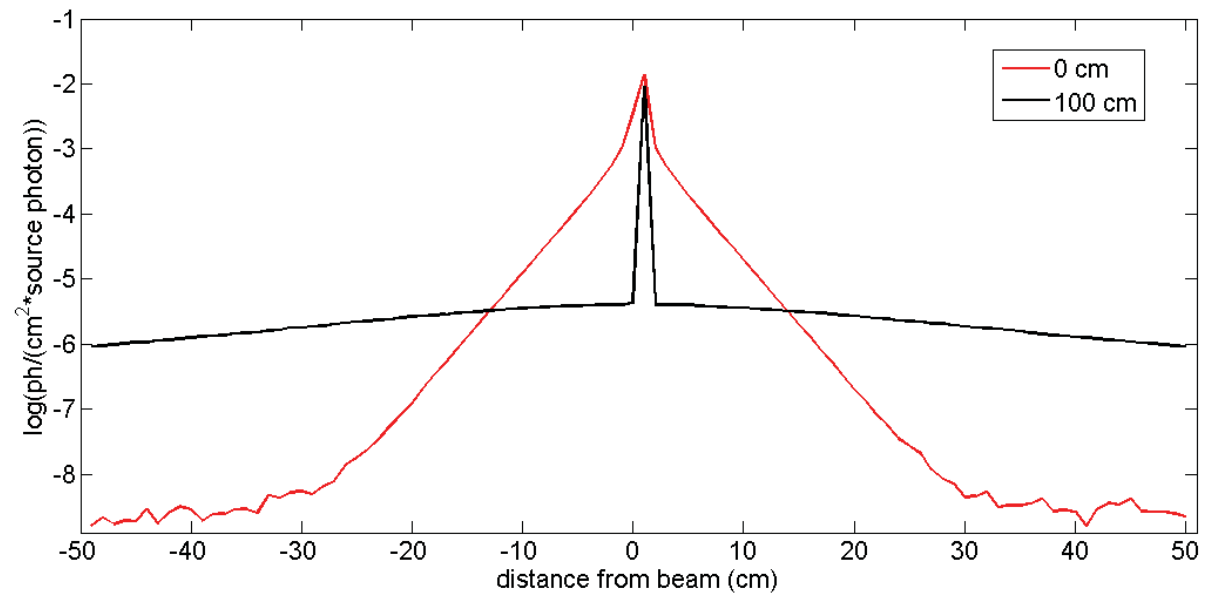

Figure 4. Flux measured in row of voxels for a $9 \mathrm{MeV}$ photon beam penetrating $20 \mathrm{~cm}$ thick steel, measured at the exit of the steel slab $(0 \mathrm{~cm})$ and $100 \mathrm{~cm}$ downstream. 


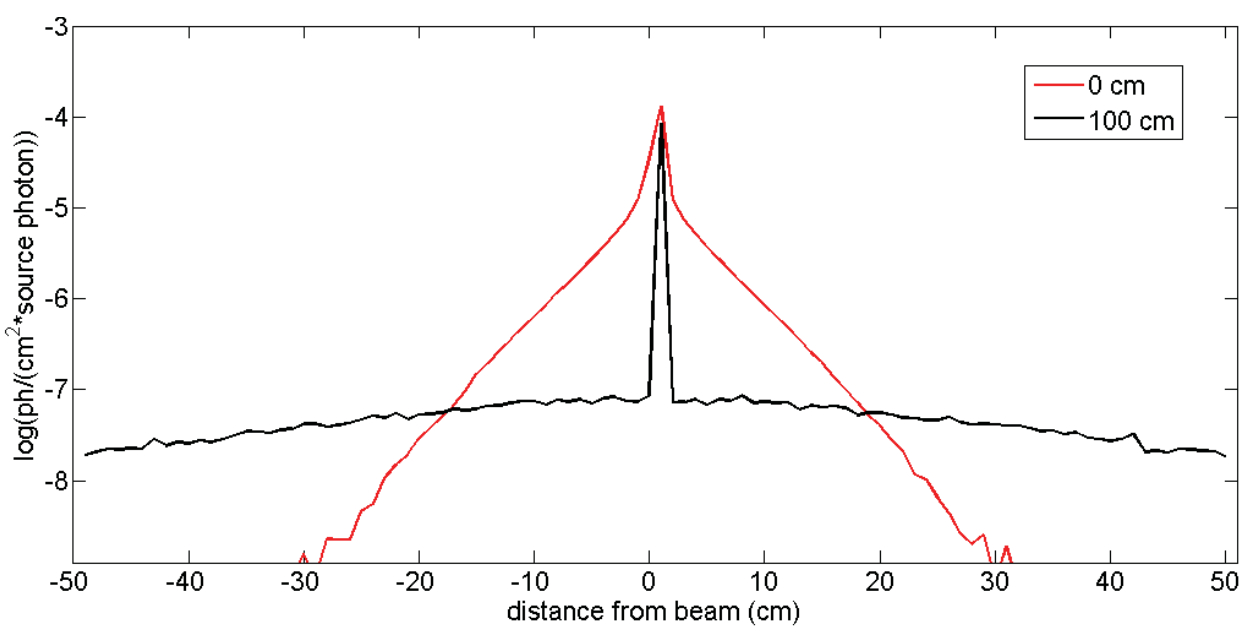

Figure 5. Flux measured in row of voxels for a $9 \mathrm{MeV}$ photon beam penetrating $40 \mathrm{~cm}$ thick steel, measured at the exit of the steel slab $(0 \mathrm{~cm})$ and $100 \mathrm{~cm}$ downstream.

The fraction of the photons observed in detector pixels outside the transmitted beam location can be used to give a qualitative first indication of the effects of the observed scatter on fan or cone collimated beams. For narrow fan beams $(1 \mathrm{~cm}$ wide in the out-of-plane direction) this estimate would be at the $3-10 \%$ level. For broader fan beams or cone beams the contrast degrades rapidly-a fan 10 to $20 \mathrm{~cm}$ wide (corresponding to a fan angle of $\sim 2$ degrees) would have scattering approaching the transmitted signal, for example. This qualitative picture serves as a guide to interpreting detailed simulations of scattering. Note that the bremsstrahlung spectrum is severely hardened by the thick steel, such that low-energy photons are suppressed. The differences, and thus the scatter contributions, will be larger for more extended, lower density objects as demonstrated below.

\section{Simulations of Radiographic Imaging Including Scattering}

Scatter contributions were assessed using Monte Carlo simulations in imaging a $5 \mathrm{~cm}$ thick lead object obscured by a $20 \times 20 \times 20 \mathrm{~cm}$ thick steel plate at the center of a container. Two types of narrow divergence-angle ("pencil") beams used in raster scan mode were evaluated: 3, 6 and $9 \mathrm{MeV}$ MPS beams as well as 6 and $9 \mathrm{MeV}$ bremsstrahlung beams. These were compared to fan and cone collimated beams to assess the contributions of energy spectrum and collimation to imaging performance. To gauge the effect of scatter on image quality, the contrast between two pixels located behind the steel plate and behind the Pb block, respectively, was calculated. The contrast $\mathrm{C}$ is defined by:

$$
\mathrm{C}=\phi_{2}-\phi_{1} /\left(\phi_{2}+\phi_{1}\right)
$$

where $\phi_{1}$ is the total fluence in a pixel obscured by the steel plate and the lead object, and $\phi_{2}$ is the total fluence in a pixel behind the steel plate.

Preliminary results reported previously [1] indicated that under identical narrow divergence beam emission angle conditions of 2 mrad, the contrasts obtained with an MPS and a bremsstrahlung beam of the same energy were comparable. Contrast in all cases was close to 0.9, when penetrating a $20 \times 20 \times 20 \mathrm{~cm}^{3}$ steel plate. Conversely, for a $9 \mathrm{MeV}$ bremsstrahlung cone beam with a relatively small 2.53 degree half-angle in combination with an uncollimated detector, the contrast was found to drop significantly to $\sim 6 \%$. The cone opening angle was chosen to fully cover the $20 \times 20 \times 20 \mathrm{~cm}^{3}$ steel plate. The severely lower contrast in this case would require much higher dose-on the order of $100 \times$ higher than that from narrowly collimated sources (MPS 
or bremsstrahlung) - to form an adequate image. This indicates that scatter control through collimation of the beam is dominant over the effects of source energy spread. This is consistent with the beam propagation simulations shown in the previous section. Indeed, narrowdivergence bremsstrahlung beams are currently known to provide strong benefit in applications where contrast is important and low throughput (due to discarding almost all the photons) is not a concern [68]. An intrinsically narrow angle MPS (e.g., Thomson) could hence provide similar benefit without sacrificing scan rate. Note that even the "broad" 2.5 degree cone angle used here is about $1 / 10^{\text {th }}$ of the angle required to subtend the whole container vertically. This indicates that even modest emission angles can significantly degrade contrast and increase dose required (regardless of source spectrum). These initial estimates motivated simulation of specific source and detector collimation geometries, source parameters, and targets to provide realistic evaluation of the parameters that control scattering and of the dose required to form an image.

Several factors that might contribute to the dramatic change in contrast, depending on beam shape, were evaluated using simulations. First, the $9 \mathrm{MeV}$ bremsstrahlung cone beam described above (2.53 degree half-angle) was simulated in detail, using a spectrum hardened by $1 \mathrm{~cm}$ of W. The initial setup had a source $200 \mathrm{~cm}$ from the container wall. A vertical linear array made of 220 $\mathrm{CdWO}_{4}$ detectors was located $80 \mathrm{~cm}$ from the container exit wall and each element was $1 \times 1 \times 3$ $\mathrm{cm}$. The array was not collimated. Then, parameters were modified in succession, including elements of the geometry of the problem, source and collimation characteristics, and parameters such as the lead cube dimensions, the beam hardness or the photon transport energy cut-off. This allowed systematic evaluation of the factors contributing to image contrast. Results are reported in Figure 6. The thin and thick lines in Figure 6 represent the unscattered and total photon intensities incident on the face of the linear array. The plot exhibits three distinct regions centered around the origin and related to the pixel position with respect to the steel plate and the lead cube. Pixels in the central region are behind the steel and lead; hence fewer photons are transmitted and intensities are the lowest. Pixels directly on each side of the central region are behind the steel plate. The contrast between these two regions is the desired performance metric, representing the acuity with which the lead cube can be discerned behind the steel plate. Successive regions further from the axis include pixels in the direct line of sight of the cone beam, which have the highest intensities. Finally, the outer pixels tally scattered photons.

Figure 6 reports the results of successive simulations varying parameters to assess contributions to image quality. The first simulation (black curve) includes a $5 \times 5 \times 5 \mathrm{~cm}$ lead block, $2 \mathrm{~mm}$ thick container walls, and a photon transport energy cut-off of $200 \mathrm{keV}$. The second simulation (blue curve) shows the effect of lowering the energy cut off to $10 \mathrm{keV}$. For this case and the other cases in Figure 6 the geometry of the lead block is similar, at $4 \times 4 \times 5.81 \mathrm{~cm}$. Next, the bremsstrahlung spectrum was changed to a non-hardened spectrum, lowering the average energy (red curve). None of these parameters affects contrast between the central region (lead) and adjacent pixels greatly. Then, the detector was moved closer, to $30 \mathrm{~cm}$ from the container, and the container wall thickness was increased to $5 \mathrm{~mm}$, which increases scattering (green curve). This noticeably degrades performance, as expected. Finally, a vertical fan beam collimation was applied to limit the beam horizontal width to $1.27 \mathrm{~cm}$ at the center of the container $(0.2$ degrees, narrow for a fan beam collimated system). This is plotted in the right panel (yellow line). While overall the unscattered component remains fairly unchanged, the total fluence shows a strong dependence on the parameters described here, leading to a contrast ranging from 0.2 for the initial simulation to 0.06 for the worst-case scenario (green curve, which includes the thicker wall). This indicates that detector position and energy cuts contribute modestly to contrast in this case. The latter is similar to the results presented previously. Container wall thickness is important as it contributes to scattering. Contrast is increased to 0.5 for the fan beam, indicating that source collimation is the dominant method to improve contrast. 

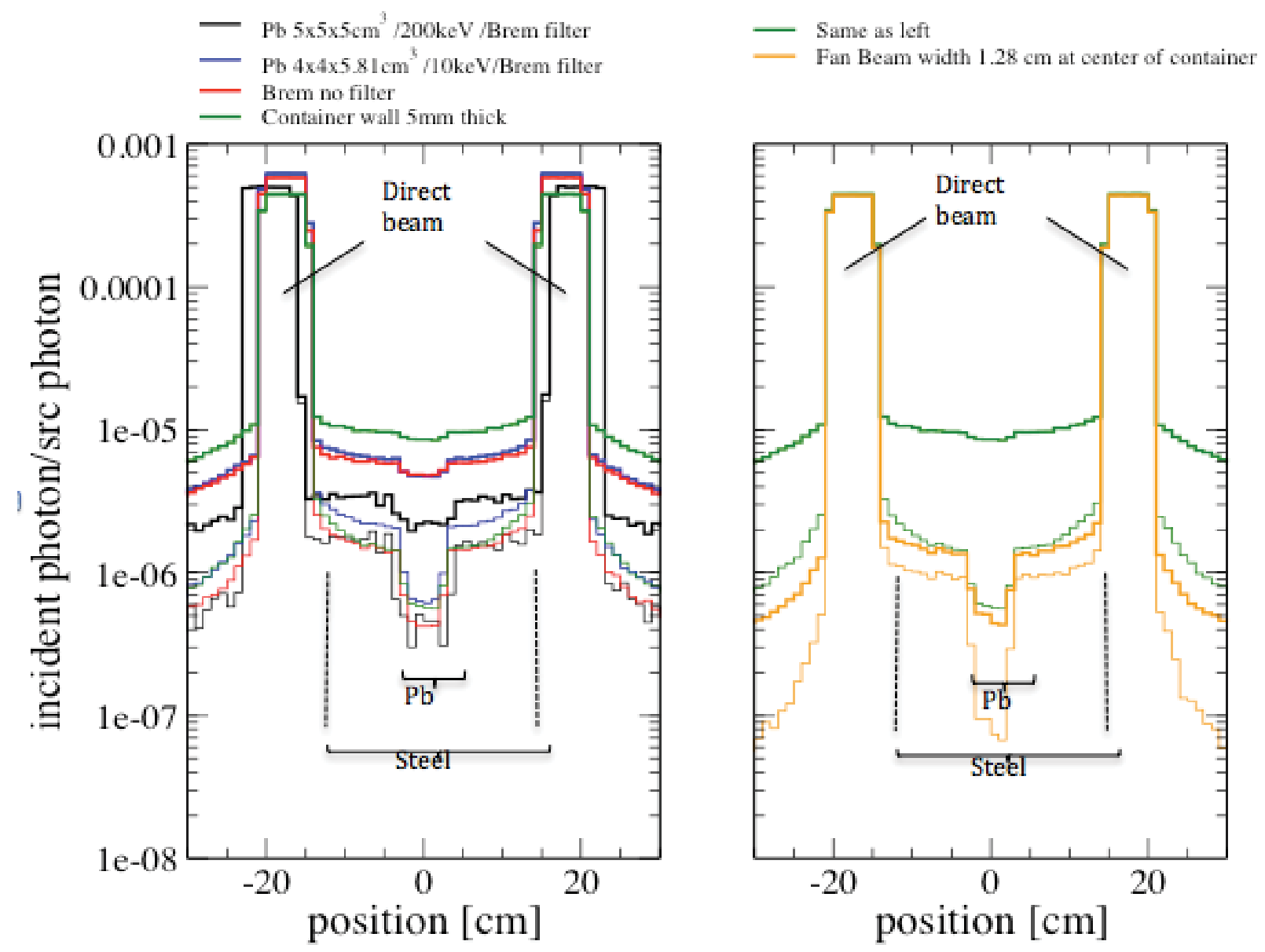

Figure 6. Fluence at the linear detector array for a $9 \mathrm{MeV}, 2.5$ degree cone beam on a $20 \times 20 \times 20 \mathrm{~cm}^{3}$ steel plate concealing an approximately $5 \times 5 \times 5 \mathrm{~cm}^{3} \mathrm{~Pb}$ cube for no collimation (left) and comparing no collimation to fan beam collimation (right). Thin lines represent unscattered fluence and thick lines total fluence. Non-zero contributions to the unscattered component outside of the beam are due to unscattered secondary particles.

A further set of simulations was conducted to emulate state-of-the-art systems that use a fan bremsstrahlung beam narrowly collimated in the horizontal axis, combined with collimated detectors, to reduce scattering contributions. These were compared to narrow-angle MPS beams. The geometry is shown in Figure 7. Simulations for a small $20 \times 20 \times 20 \mathrm{~cm}$ steel plate were compared to those for a large steel plate $300 \mathrm{~cm}$ wide $\times 238 \mathrm{~cm}$ high $\times 20 \mathrm{~cm}$ thick, each located at the center of a cargo container. The linear array of 220 detectors, $1 \times 1 \times 3 \mathrm{~cm}$, is collimated in the horizontal direction by two tungsten (W) plates extending $30 \mathrm{~cm}$ from the front face of the detectors. This detector geometry was chosen to limit any contribution from secondary scatter coming from the sides of the cargo and container. Two configurations were modeled, each for 6 and $9 \mathrm{MeV}$ bremsstrahlung end-points. In the first, the source was at a distance of $3.8 \mathrm{~m}$ from the container. The bremsstrahlung source had a fan beam vertical opening of 17 degree half-angle to cover the container, and a horizontal opening angle set to achieve a $2 \mathrm{~cm}$ width at the center of the container and $4 \mathrm{~cm}$ at the detector. The detector was $3.8 \mathrm{~m}$ from the container, and container walls were $5 \mathrm{~mm}$. The long separation distance mitigates scatter, but is not representative of many systems in use. The second configuration had the source closer to the container at $2 \mathrm{~m}$. The bremsstrahlung source vertical fan angle was increased to a 30 degree half-angle to cover the container, and the horizontal beam collimation was reduced to deliver a $1.0 \mathrm{~cm}$ beam width at the 
detector. The detector was $0.8 \mathrm{~m}$ from the container and the container walls were $2 \mathrm{~mm}$ thick. These source and detector distances are closer to systems in use, although source collimation may be narrower than commonly used in developed systems [5]. For comparison to the bremsstrahlung beams, 3 and $9 \mathrm{MeV}$ monoenergetic photon sources (energies chosen for best $\mathrm{Z}$ discrimination based on mass attenuation coefficient) were modeled. Each MPS had a $2 \mathrm{mrad}$ full divergence, corresponding to a $\sim 0.6 \mathrm{~cm}$ width at the container center (derived from realistic parameters for a Thomson source). Simulations were run for the MPS and fan beam using the same source and detector locations. While the MPS location is fixed, the direction of its narrowdivergence beam was scanned to aim at a specific pixel, as shown in Figure 7.

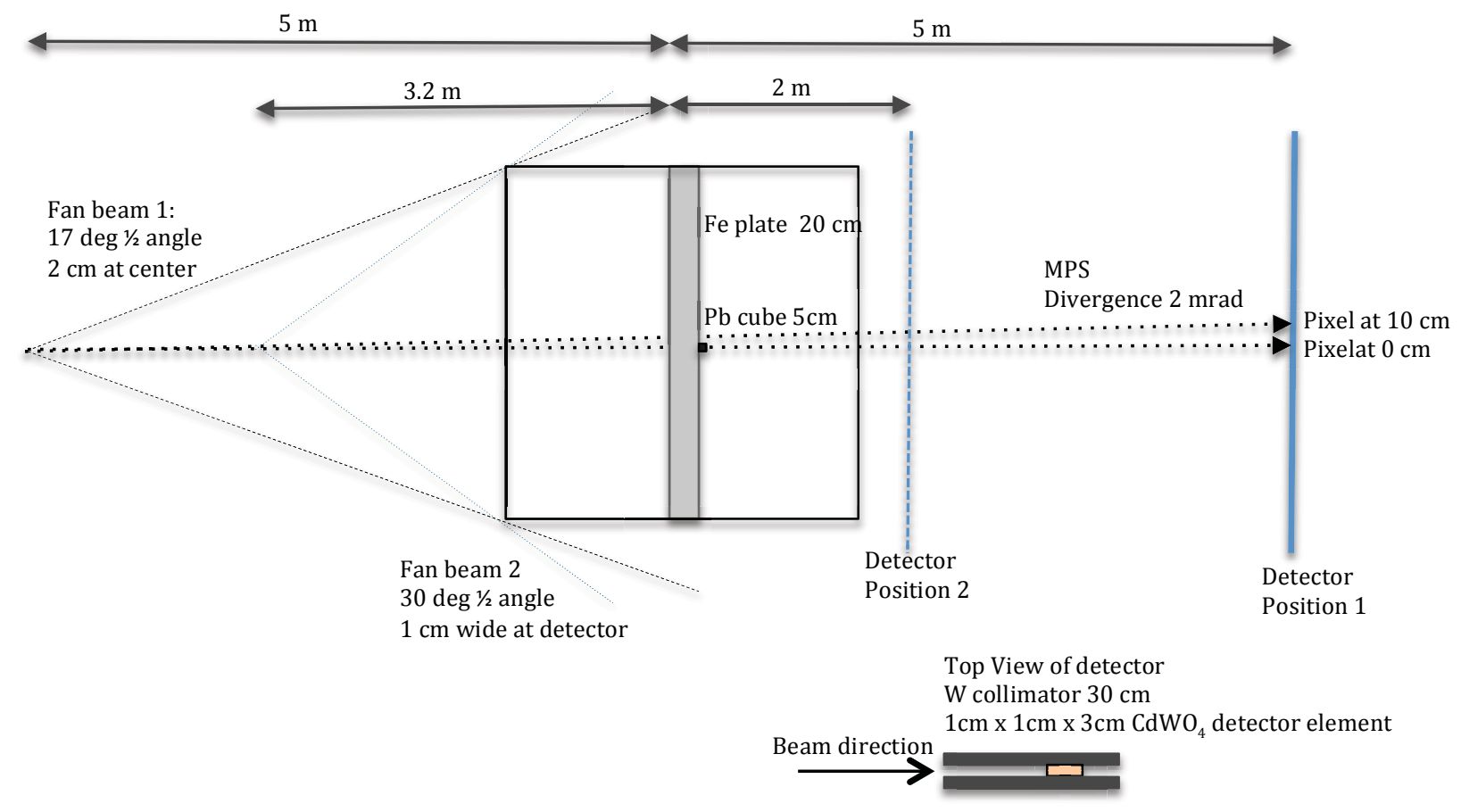

Figure 7. Geometry of the problem: the cargo container. Overall size is $6 \times 2.4 \times 2.4 \mathrm{~m}$. The source is $10 \mathrm{~m}$ from the detector in configuration 1 , and $5.2 \mathrm{~m}$ in configuration 2 . A top view of a detector element inside the collimator is shown in the bottom right corner.

Photon fluences incident on the detectors are larger for the small steel plate than for the large one, as shown in Figure 8. The large steel plate acts as a beam absorber, while in the case of the small steel plate, a large fraction of the fan beam illuminates the $5 \mathrm{~mm}$ steel walls and scatters in the detectors. For the small plate case, the contrast is 0.76 . However, when container walls are removed the contrast increases to 0.82 , essentially equal to the value of 0.83 obtained for the large plate case reported in Table 10. The container walls can be considered as stand-ins for other scattering sources in a container. The strong dependence on the container walls indicates that for the fan beam collimated source (MPS or bremsstrahlung), contrast can be sensitively dependent on surrounding material. This effect becomes more severe the wider the collimator angle, as indicated by the $2.5^{\circ}$ cone results above. This is not the case for a pencil-beam collimated source, where contrast contributions due to scattering are readily isolated (see Figures 3, 4, 5). Finally, in the large plate configuration, the contrast achieved with the MPS and bremsstrahlung beams going through $20 \mathrm{~cm}$ steel is high, as seen in Figure 9. The pencil-beam collimated MPS in this case shows only a small improvement, from 0.83 to 0.88 . Bremsstrahlung simulations through 40 $\mathrm{cm}$ of steel were prohibitive. However the MPS simulations yielded contrast similar to those observed for the thinner plate, albeit with a signal smaller by at least two orders of magnitude. 
While the observed contrast improvements due to use of a pencil-beam MPS in these cases over a very narrow fan beam were modest, the dependence of the fan beam results on scattering motivated further investigation of cases where scattering effects are strong.

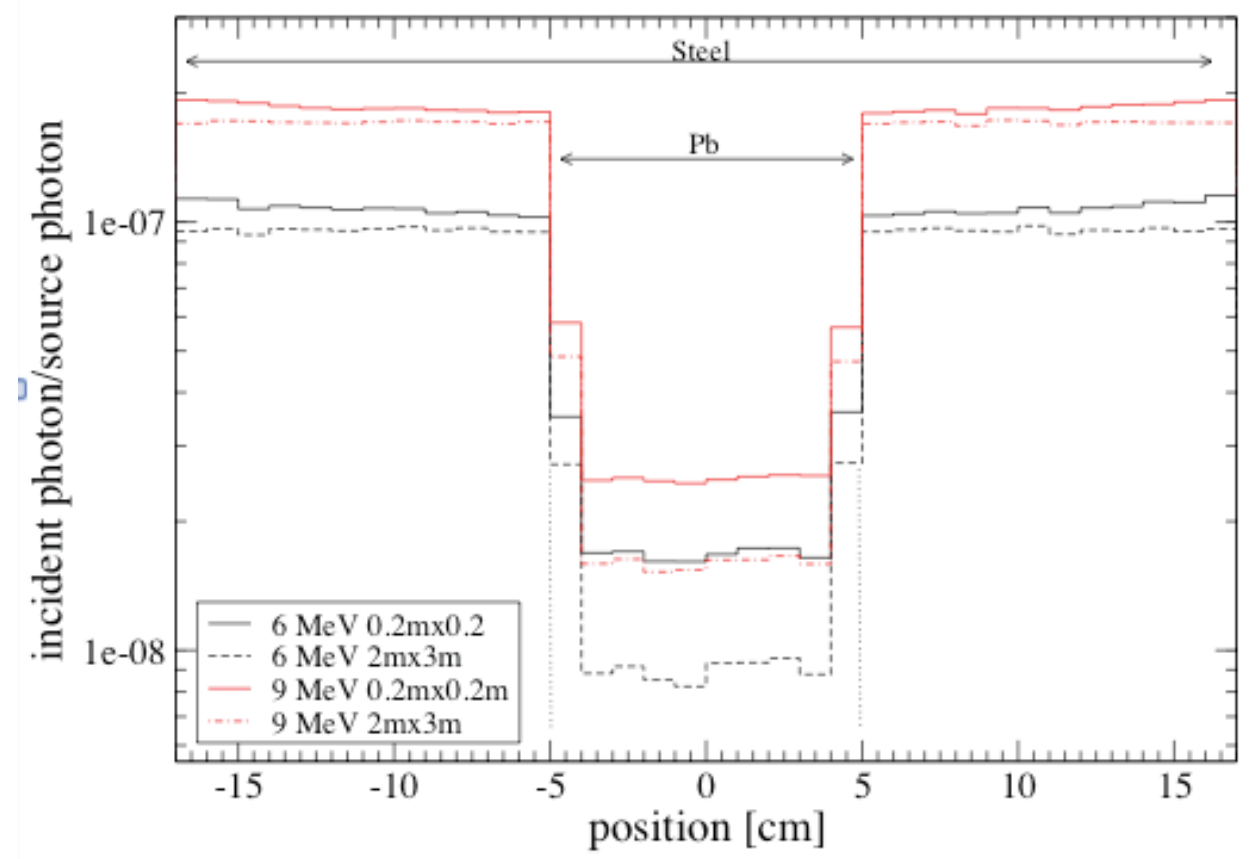

Figure 8. Total photon intensity on the linear array of detectors for $6 \mathrm{MeV}$ (black) and 9 (red) MeV bremsstrahlung beams. The steel plates are $20 \mathrm{~cm}$ thick by $20 \times 20 \mathrm{~cm}$ (full lines) or $300 \times 238 \mathrm{~cm}^{2}$ (dotted lines). The cargo central axis is at the origin; hence, pixels in the center part of the plot are behind $20 \mathrm{~cm}$ of steel and $5 \mathrm{~cm}$ of lead. The detector is located $5 \mathrm{~m}$ from the center of the container and the system has a magnification of 2 .

Table 10: Estimated contrast for a source-to-detector distance of $10 \mathrm{~m}$ (configuration 1) and $5.2 \mathrm{~m}$ (configuration 2). MPS vs. bremsstrahlung beams filtered by $1 \mathrm{~cm} \mathrm{~W}$, incident on a large steel plate and a $5 \times 5 \times 5 \mathrm{~cm} \mathrm{~Pb}$ cube.

\begin{tabular}{|l|l|l|l|l|}
\hline & \multicolumn{4}{|c|}{ Contrast: $20 \mathrm{~cm}$ Fe } \\
\hline Distance & $3 \mathrm{MeV}$ MPS & $9 \mathrm{MeV}$ MPS & $6 \mathrm{MeV}$ Brem & $9 \mathrm{MeV}$ Brem \\
& & & & \\
\hline $10 \mathrm{~m}$ & 0.83 & 0.88 & 0.83 & 0.83 \\
\hline $5.2 \mathrm{~m}$ & 0.79 & 0.84 & 0.81 & 0.81 \\
\hline
\end{tabular}

\begin{tabular}{|l|l|l|l|l|}
\hline & \multicolumn{4}{|c|}{ Contrast: $40 \mathrm{~cm}$ Fe } \\
\hline Distance & $3 \mathrm{MeV}$ MPS & $9 \mathrm{MeV}$ MPS & $6 \mathrm{MeV}$ Brem & $9 \mathrm{MeV}$ Brem \\
& & & & \\
\hline $10 \mathrm{~m}$ & 0.82 & 0.88 & - & - \\
\hline $5.2 \mathrm{~m}$ & 0.80 & 0.84 & & \\
\hline
\end{tabular}




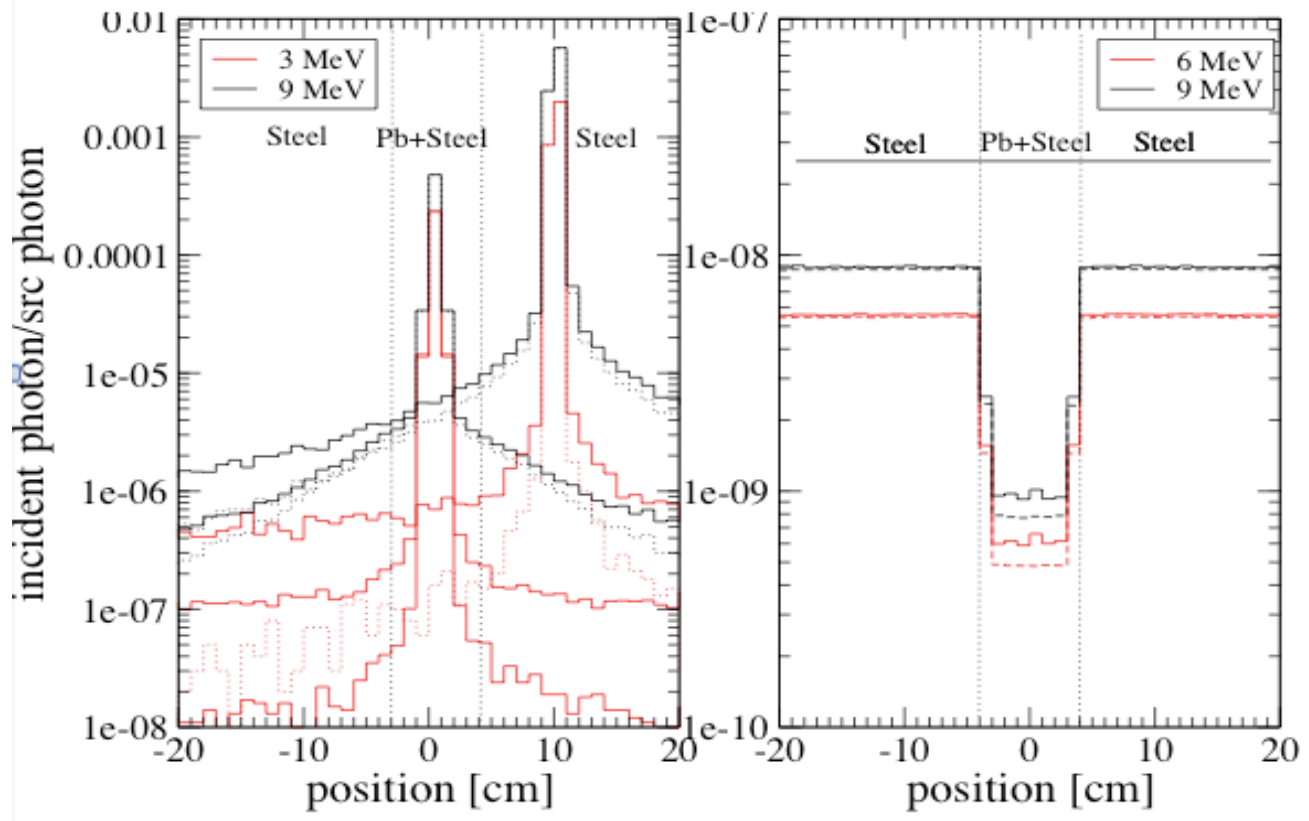

Figure 9. Photon fluence incident on linear array of detectors for 3 and 9 MeV MPS pencil beams (left, showing example pencil beam locations) and 6 and $9 \mathrm{MeV}$ bremsstrahlung fan beams (right, showing full fan line which convolves scattering). The beam penetrates through $20 \mathrm{~cm}$ of steel at position $x=10 \mathrm{~cm}$ and $20 \mathrm{~cm}$ of steel and $5 \mathrm{~cm}$ of lead at position $x=20 \mathrm{~cm}$. Unscattered (dotted line) and total fluences (thick line) are shown.

In order to further investigate the effect of scattering material surrounding a highly attenuating region, we simulated the imaging of a $5 \times 5 \times 5 \mathrm{~cm} \mathrm{~Pb}$ cube behind a small $20 \times 20 \times 20 \mathrm{~cm}$ steel plate with a narrow $9 \mathrm{MeV}$ bremsstrahlung fan beam for the same configuration as described above, only having the remainder of the container filled with water at a density of 0.2 $\mathrm{g} / \mathrm{cm}^{3}$ [69]. The result with a narrowly collimated $9 \mathrm{MeV}$ bremsstrahlung fan beam is shown in Figure 10. The unscattered photon intensity is a factor of $\sim 10$ greater behind the steel plate than behind the steel and $\mathrm{Pb}$, and the unscattered contrast would be $\sim 0.83$. The total photon intensity, on the other hand, , differs by only a factor of 2 due to scatter in the water surrounding the steel and $\mathrm{Pb}$, and the contrast is hence reduced to $\sim 0.3$ by the effects of scattering. This is in contrast to the case without water, which showed only modest contrast degradation. Scanning with an MPS pencil beam would eliminate the contribution from scatter in the water and preserve high contrast. A factor of a few less dose would be required using the pencil to achieve the same image quality achieved with the fan beam. 


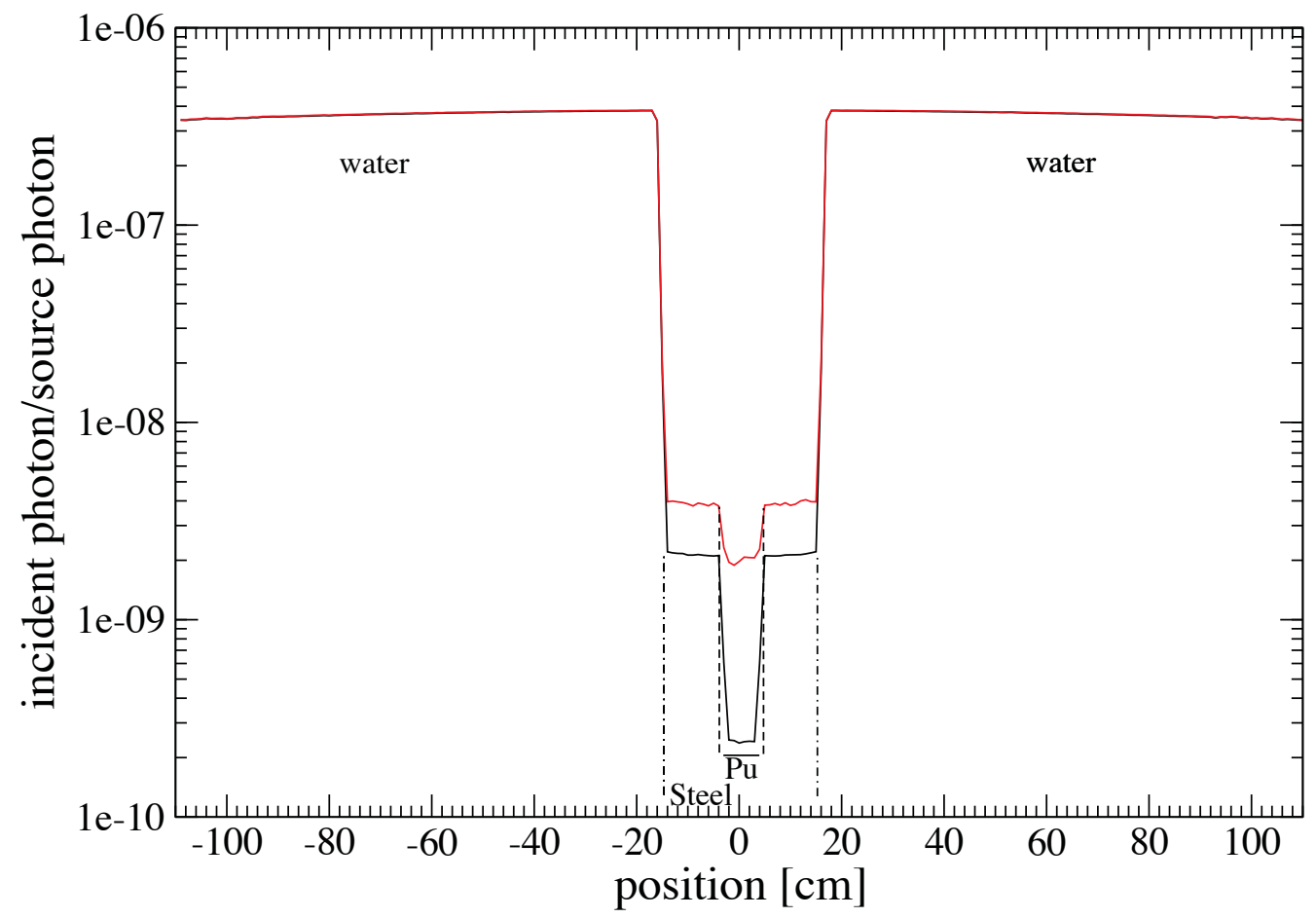

Figure 10. Photon fluence incident on linear array of detectors for a $9 \mathrm{MeV}$ bremsstrahlung source penetrating through a $20 \times 20 \times 20 \mathrm{~cm}$ steel plate and $5 \times 5 \times 5 \mathrm{~cm}$ lead cube surrounded by water. The unscattered (black line) and total fluences (red line) are shown. A pencil beam would eliminate the scattering effect, essentially reproducing the black line.

These cases underscore the fact that contrast variations for a fan beam are strongly dependent on the configuration of the cargo load as well as the structures and components of the radiographic system surrounding the container that will be in the path of the fan beam, rendering the identification of a high-Z material region difficult if it is embedded behind thick material with a $\mathrm{Z}$ similar to steel or surrounded by scattering sources. Both contributions can be greatly reduced by using a narrow-divergence MPS, since for such a beam first-order scatter from the cargo, container and radiographic system does not contribute to the image, and second-order scatter is not significant. Schematic simulations with a single $20-40 \mathrm{~cm}$ thick steel plate indicate that for the configurations without the extra low-Z (water) scatterer, the use of 3 and $9 \mathrm{MeV}$ narrow-angle MPSs only yields a small contrast improvement compared to 6 and $9 \mathrm{MeV}$ narrowly collimated bremsstrahlung fan beams. However based on the documented variety of goods being shipped and of cargo loading configurations, during actual cargo imaging at ports of entry it is expected that the scatter component for a fan beam system can be much larger, depending on cargo and support structure configurations. This will reduce contrast, similar to the scattering effect demonstrated in Figure 10. The simulations show that, as long as transmission is sufficient, a narrow angle MPS (e.g., a Thomson source) is insensitive to this effect, offering a significant advantage for cargo applications. In cases where scattering is severe, such a source may significantly reduce the dose required to form an image. Other MPS candidates, such as nuclear reaction sources, can be considered to be nearly $4 \pi$ in emission. Scenarios with a source illuminating two containers, one on each side of the source, have been proposed. Unlike the narrow angle MPS and fan beam, cargo scatter cannot be reduced by controlling the spatial resolution of the beam in this case. Use of multiple collimators and/or tight collimation of the detectors combined with energy threshold need to be evaluated to help extract information from 
such an imaging system. Such a technique is being actively investigated and evaluated against existing systems [70].

\section{Enhancement of Z Discrimination Using Dual Energies}

The ability to isolate high-Z $(>72)$ objects in radiography of cargos is a key need. This has remained a challenging problem so far. Current systems have to contend with convolution of two broad bremsstrahlung spectra and with scattering due to the fan beam collimation [71]. Assuming a low divergence beam traversing a slab of material and a negligible scatter contribution, the low-Z/high-Z contrast in a dual energy measurement is highest if one energy is around $4 \mathrm{MeV}$ and the other one at the upper energy limit. For a bremsstrahlung source the corresponding endpoint energies are $6 \mathrm{MeV}$ and $9 \mathrm{MeV}$ ( $9 \mathrm{MeV}$ is close to the upper energy limit for cargo screening). The energy range for MPSs (in particular based on Thomson scattering) is not limited by the bremsstrahlung spectrum and hence 8-9 $\mathrm{MeV}$ and 3-4 MeV monoenergetic beams are appropriate for contrast.

Initial evaluation indicated that significantly better $\mathrm{Z}$ discrimination can be achieved with a narrow energy spread beam, measuring transmission at several well defined energies, than with a bremsstrahlung beam that integrates transmission over the full energy range and may experience spectral change when traversing the cargo. The difference in the transmission ratios between two energies for lead vs. steel in simple calculations was roughly three times greater for an MPS versus bremsstrahlung. Narrow energy distribution, narrow beam divergence MPSs with the ability to select and rapidly switch energy over the full range of 1-10 MeV could hence potentially enable the development of improved systems. This motivated quantitative simulation to assess potential benefit.

Simulations to detail the capabilities of an MPS-based system first evaluated effects of photon energy spectrum (neglecting scattering) on $\mathrm{Z}$ resolution, and then considered the effects of scattering on $\mathrm{Z}$ resolution. Two sets of targets were assessed: those defined for the CAARS ATD, and a simple geometry commonly used in the past. The CAARS ATD program defined a set of challenging objects for tests of $\mathrm{Z}$ discrimination. Tested systems, which did not include MPSs, had high false alarm rates and/or inconsistent automatic detection of high-Z objects possibly caused by scatter in some cases [71]. To investigate the potential benefits for $\mathrm{Z}$ discrimination of an MPS dual energy system, and the appropriate energies to use, simulations were conducted using the CAARS objects. Z Additionally, a simple cube-behind-plate target consisting of $\mathrm{Pb}$ cubes hidden behind steel plates was also used for assessment of $\mathrm{Z}$ discrimination and for comparison to past studies.

The production version of the HADES radiography simulation code [72], which is based solely on photon transmission and does not account for scatter, was used to evaluate Z-discrimination differences due to photon source spectrum. Images of several CAARS test objects were simulated for two types of parallel beams: MPS beams ranging from 1 to $10 \mathrm{MeV}$, and 6 and $9 \mathrm{MeV}$ bremsstrahlung beams. Attenuation ratio maps were calculated for energy pairs. Additionally, since HADES does not account for scatter in the object, Monte Carlo simulations were run for one of the objects. The combined simulations illustrate the advantages of dual-energy MPSs for Z discrimination, and the robustness of that advantage to scattering effects.

Figure 11 shows attenuation ratio maps and vertical lineouts for CAARS objects 3 and 4 . Object 3 is made of a yellowcake cylinder inside concrete. Object 4 consists of a $5 \mathrm{~cm}$ radius sphere of DU embedded in the center of a cube of lead and centered in a cube of Lexan placed at the center of a cargo container. The lead and Lexan cubes are $26 \mathrm{~cm}$ and $100 \mathrm{~cm}$ on a side, respectively. The smallest differences were observed for the $9 / 6 \mathrm{MeV}$ bremsstrahlung ratio. The best overall MPS energy combination for $\mathrm{Z}$ discrimination of the CAARS objects was found to be 3 
and $9 \mathrm{MeV}$ or 4 and $8 \mathrm{MeV}$, respectively. Notably, the 9/3 MPS lineout of the attenuation ratios show a $\sim 2 \%$ enhancement at the location of the DU sphere whereas there is no appreciable change in signal for the $9 / 6$ bremsstrahlung beams. This demonstrates the greater sensitivity of MPS dual energy imaging. For CAARS Object \#3, the relative difference in ratio between the yellow cake and concrete regions for the $9 / 3 \mathrm{MeV}$ MPS is a factor of 5 greater than that for the $9 / 6 \mathrm{MeV}$ Bremsstrahlung. What sensitivity can be achieved in an actual measurement will depend on measurement errors, systematic errors introduced by the radiographic scanning system, and photon counting statistics, but it is clear that use of monoenergetic photon beams provides a severalfold increase in contrast to offset such potential issues.
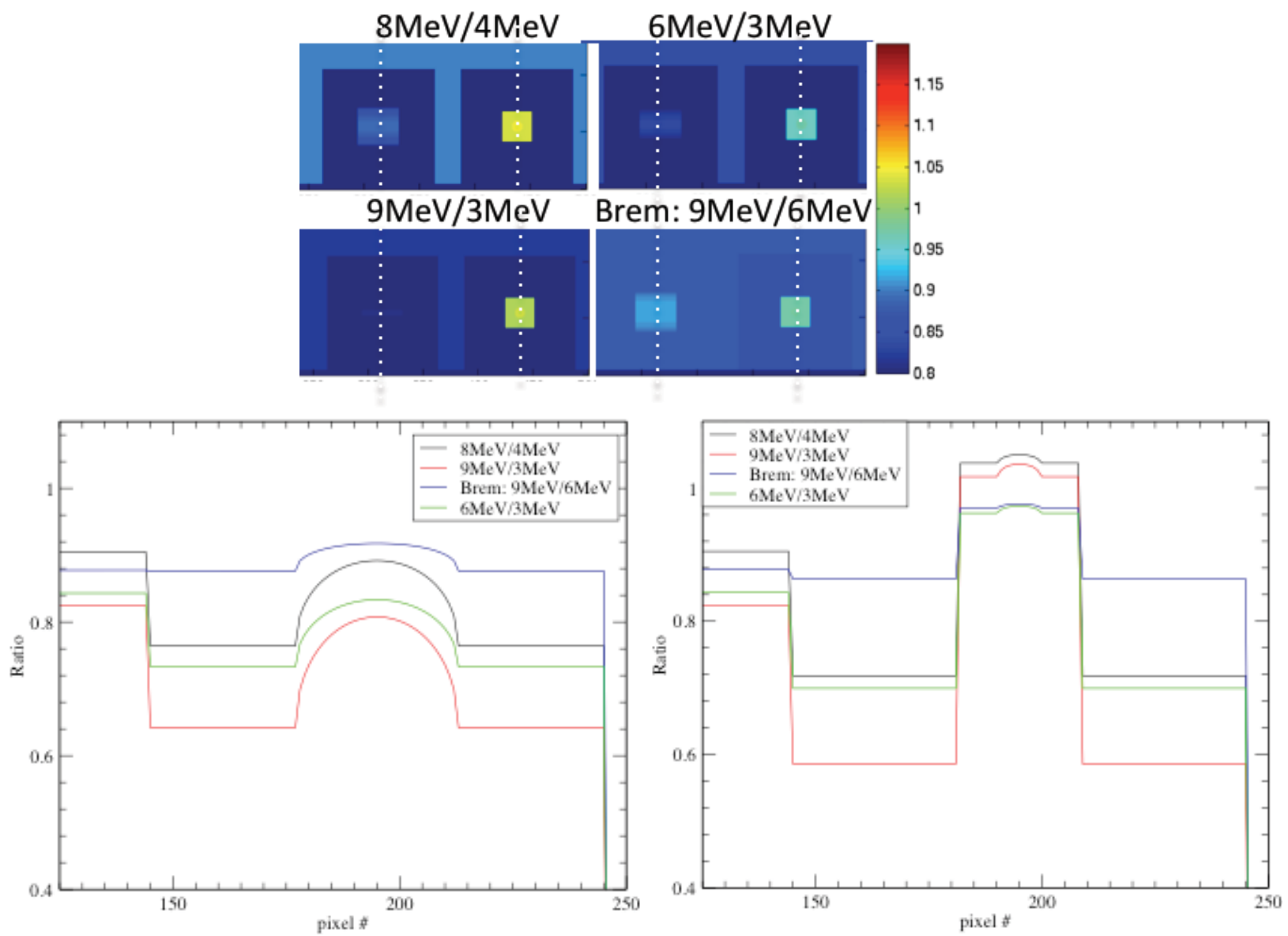

Figure 11. Images of attenuation ratios of MPS and bremsstrahlung beams through CAARS objects \#3 and 4 (top). Images were simulated at each energy with the HADES code. Vertical lineouts through the center of the objects are plotted for object 3 (bottom left) and 4 (bottom right).

To evaluate the effects of scattering on $\mathrm{Z}$ discrimination, Monte Carlo simulations were run for Object 4 in a geometry corresponding to configuration 1 in Figure 7, i.e., the distance between the source and the detector is $10 \mathrm{~m}$, resulting in a magnification of 2 . This case was chosen to determine if the DU and lead region could be differentiated using either a bremsstrahlung fan beam source or a narrow divergence MPS when scatter was included. The narrow divergence MPS was the case evaluated here (Figure 12) because the scattering simulations above showed that such a source has the best capability to mitigate scatter and hence to potentially address this challenging case. 


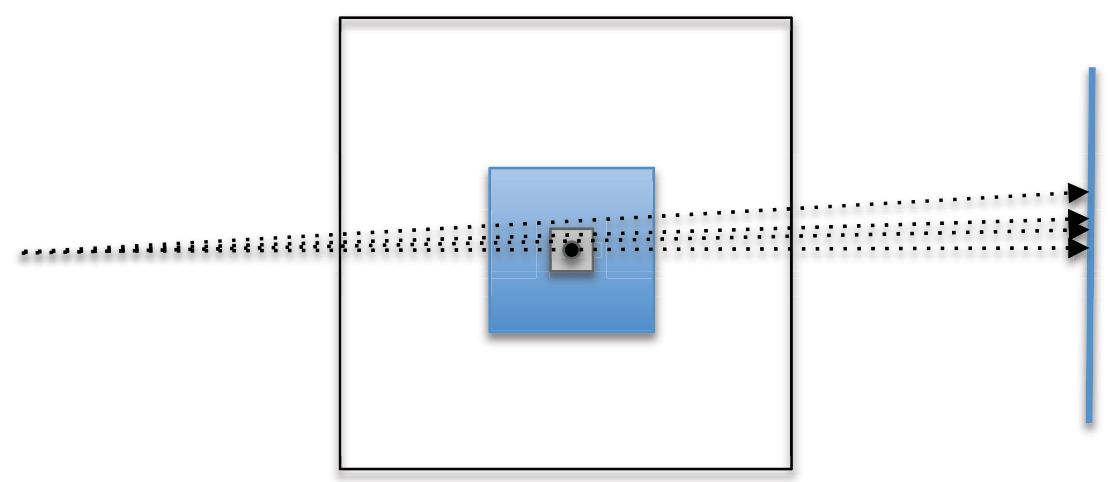

Figure 12. Simulation geometry: the narrow divergence beams are emitted from the same location; each beam is angled to go through a different region of object 4 (blue square).

Both the 6 and $9 \mathrm{MeV}$ bremsstrahlung fan beam simulations show no differentiation between the $\mathrm{U}+\mathrm{Pb}$ and $\mathrm{Pb}$ regions in the center. This is not surprising, given that the contrast without scatter was already very small in this region (above). Scattering in the thick target is significant, causing loss of any signal. The signal does increase by two orders of magnitude in the region behind the Lexan, due to the much higher transmission in this area. The transmitted flux lineouts are plotted in Figure 13.

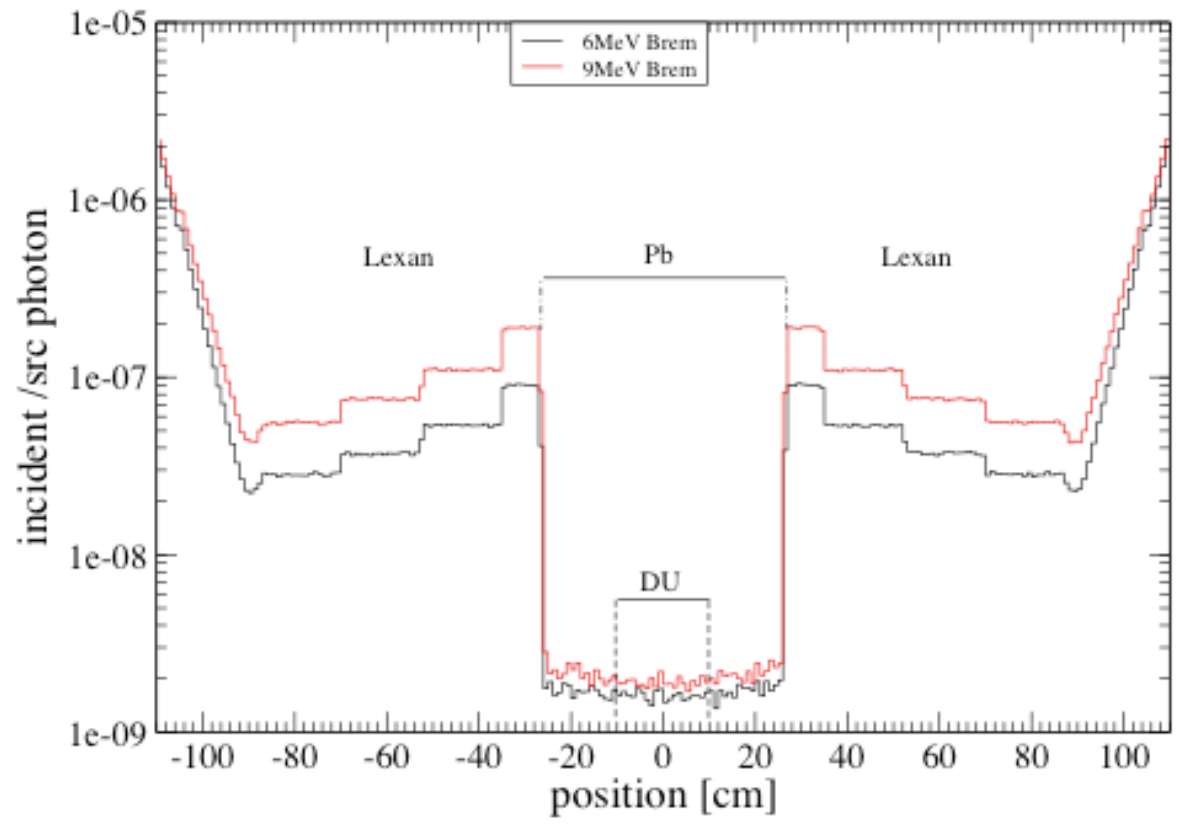

Figure 13. Simulated transmitted intensity including scattering for CAARS Object 4 using 6 and 9 bremsstrahlung fan beams, as detected on a linear array of $1 \times 1 \mathrm{~cm}$ detectors.

Simulations were next conducted for 3 and $9 \mathrm{MeV}$ MPSs with narrow divergence pencil beams, the setup shown in Figure 12. Each line in Figure 14 shows the detected signal for a particular aiming of the pencil beam. The MPS is directed at the center of the object (black), at the Pb region (green) and at the low-Z Lexan region (blue). There is little signal when the beam aims at a pixel located behind the center of the DU sphere since photons are attenuated by $10 \mathrm{~cm}$ of DU and 16 $\mathrm{cm}$ of $\mathrm{Pb}$ (black curves). The signal is significantly stronger for a beam going through the $\mathrm{Pb}$ but not the DU (green curves). The blue curves correspond to the MPS beam aimed at the low Z 
region made of Lexan. While scattering is severe, a transmitted peak is still visible in all cases. The intensity in the illuminated pixel behind the lead is low, of order of a few $1 \times 10^{-8}$ photons/source photon. Assuming the maximum beam intensity of $1 \times 10^{8}$ photons per pulse, a signal of a few counts per shot could be generated during each shot. Multiple high intensity shots could be aimed at the same pixel with a $\mathrm{kHz}$ repetition rate source to obtain reasonable signal-to-noise ratio. The intensity behind the DU is even lower, on the order of $10^{-9}$, and scattering is severe. Many shots would be required to obtain statistics, and further detector collimation is likely important to extract a usable signal. Nonetheless, a difference is clearly visible in this simulation between the lead and DU regions, which was not the case for the fan beam (Figure 13). This indicates the power of a MPS pencil beam to access very challenging targets. The intensity per source photon in a pixel behind the low-Z Lexan is 5 orders of magnitude larger than for the high-Z regions. The ability to modulate MPS intensity on a per-shot basis would therefore be important; it would allow limiting the dose by optimizing the beam intensity on the next pixel, based on knowledge of intensities in the neighboring pixels or on an initial shot at low flux.

A separate study based on absorption cross-sections ratios at low and high energies was conducted using a geometry consisting of a lead cube behind a steel plate. This is a lower-scatter configuration than the CAARS objects and provides a comparison of $\mathrm{Z}$ discrimination in a configuration used in the cargo radiography evaluations discussed in the previous section. The ability to estimate $\mathrm{Z}$ from total transmitted intensities was compared for 3 and $9 \mathrm{MeV}$ MPS pencil beams and for 6 and $9 \mathrm{MeV}$ bremsstrahlung narrow fan beams using the configuration of a $\mathrm{Pb}$ cube behind a $20 \mathrm{~cm}$ thick steel plate as seen in Figure 7 with the detector in position 2 . Transmitted intensities were obtained for two beam paths, either through steel or through steel and lead, and the ratios of the total transmitted intensities were taken for the two beam energies. The resulting ratios are listed in Table 11. Quite strikingly, the ratios for the bremsstrahlung beams are indistinguishable within the statistical uncertainties $(\sim 3 \%)$ of the simulation, whereas the MPS ratios differ by about 30\%, indicating an improvement of approximately tenfold.

It should be noted that separate work by Martz et al. published during the course of this study also compared material discrimination contrast obtained with two dual-energy systems. A 3.5 and $9 \mathrm{MeV}$ MPS was compared against a 6 and $9 \mathrm{MeV}$ bremsstrahlung system, for plates made of low-Z or high-Z materials hidden behind a $20 \mathrm{~cm}$ Fe plate. [68] In that study the plate thicknesses were chosen to provide the same low energy transmission as a plate of $5 \mathrm{~mm}$ of uranium. When compared to $\mathrm{U}$ cross-section ratios, low-Z contrast for MPS was found to be greater by at least a factor of three compared to bremsstrahlung beams, while W was indistinguishable from $\mathrm{U}$.

For the various configurations assessed in this study and by Martz et al., results clearly indicate that the sensitivity to $\mathrm{Z}$ is rather weak for a $9 / 6 \mathrm{MeV}$ bremsstrahlung system. Hence even in a low-scatter case, $\mathrm{Z}$ discrimination is unlikely to be reliable, given statistical and systematic uncertainties. Dual energy MPS ratios on the other hand are sensitive to Z. While optimal MPS performance is obtained with energy spread at the $10 \%$ level, there is little loss of performance at $20 \%$ energy spread. Furthermore, even MPSs with larger energy spreads, up to the 50\% level, could provide significant advantages over bremsstrahlung sources. In addition, scatter degradation is not significant when using pencil beams. 

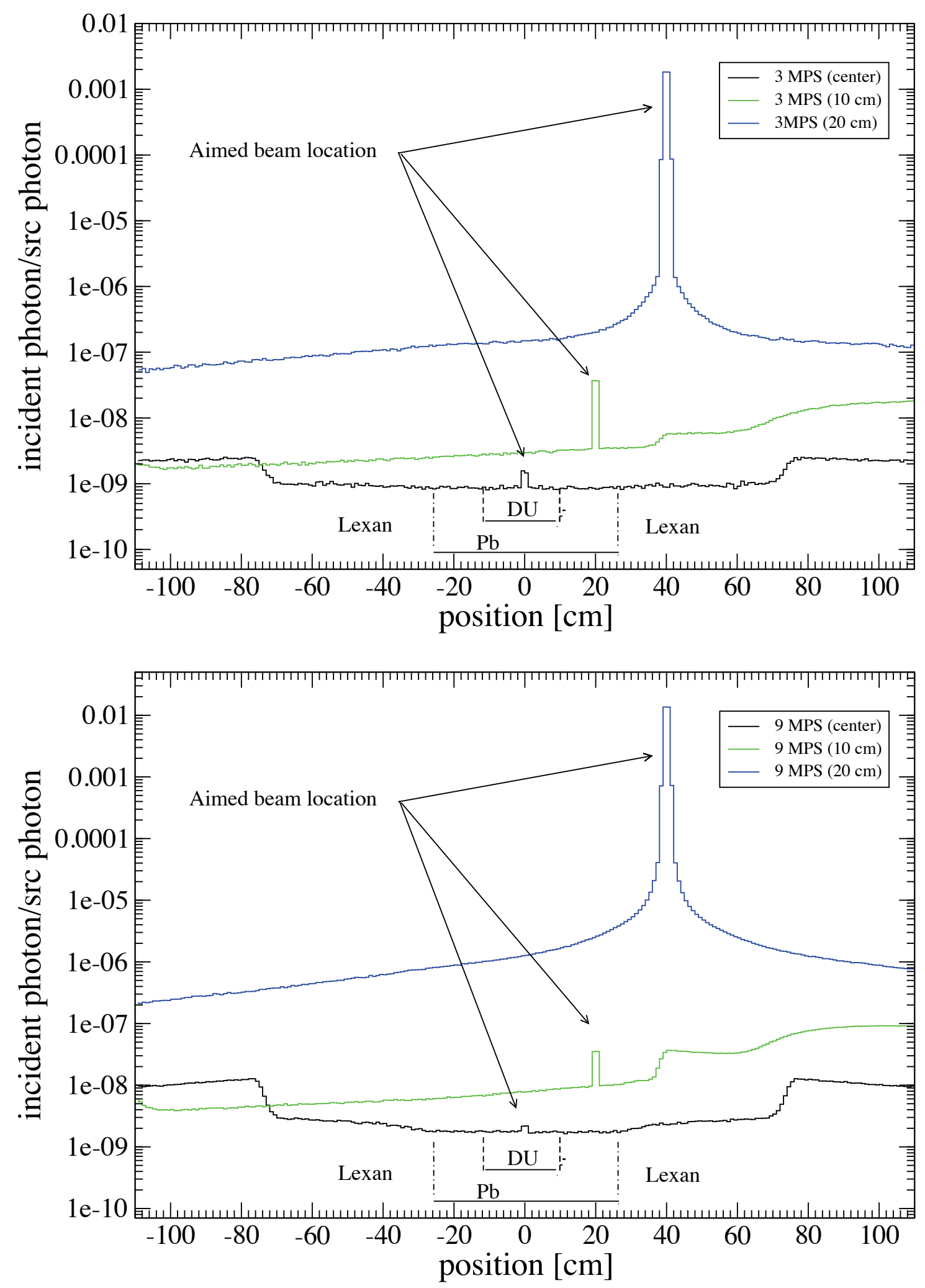

Figure 14. Simulated transmitted intensity including scattering for CAARS Object 4 using 3 MeV MPS (top) and $9 \mathrm{MeV}$ MPS (bottom) beams, as detected on a linear array of $1 \times 1 \mathrm{~cm}$ detectors. The MPS is directed at the center of the object (black), at the $\mathrm{Pb}$ region (green) and at the low- $Z$ Lexan region (blue). 
Table 11: Ratio of total counts for MPS and bremsstrahlung beams hardened with $1 \mathrm{~cm}$ of W through $20 \mathrm{~cm}$ of steel and $5 \mathrm{~cm}$ of lead. Statistical uncertainties for the bremsstrahlung results are $\sim 3 \%$.

\begin{tabular}{|c|c|c|}
\hline Beam \& Energy ratio & Steel & Steel+Pb \\
\hline Brems $9 \mathrm{MeV} / 6 \mathrm{MeV}$ & 1.57 & 1.61 \\
\hline MPS $9 \mathrm{MeV} / 3 \mathrm{MeV}$ & 2.87 & 2.04 \\
\hline
\end{tabular}

As discussed in our previous report [1], for monoenergetic beams and relatively simple geometries such as the one used in the simulations described here (a Pb cube behind a thick steel plate), the mass attenuation coefficients $\mu$ at the two energies, which are used for Zdetermination, can be calculated from measured transmission $\left(\mathrm{I} / \mathrm{I}_{0}\right)$ ratios in a straightforward manner. No a priori knowledge of the $\mathrm{Z}$, density or thickness of the materials is needed for such geometries. This may be helpful in threat detection in some situations. However, in practice, detection may require additional information be exploited in cases of irregular shapes and/or in more cluttered cargo.

An alternative $\mathrm{Z}$ discrimination and 3D imaging method, called EZ-3D, has been developed by Passport Systems [61]. This secondary bremsstrahlung-based imaging method is based on scanning with a narrow beam and gaining spatial information by measuring photons at $130^{\circ}$ to the beam axis with an array of collimated NaI detectors. Compared to transmission imaging using the dual energy method that relies on a 2D projection of a 3D object, EZ-3D has two advantages. First, it is a 3D measurement where voxelization of the cargo is set by the beam collimation and by the orientation and field of view of the detector array with respect to the beam. Second, it has a much stronger Z-dependence of $\sim Z^{3.5}$ than the dual energy method. Replacing the Rhodotron generated bremsstrahlung beam with a narrower angular divergence, higher average photon energy MPS pencil beam could potentially significantly increase the EZ-3D performance. EZ-3D requires photon energy discrimination to determine the ratio of total photon flux to the flux of photons with energies above $511 \mathrm{keV}$. However, a detection system with energy resolving detectors such as Passport's would not be suitable for a Thomson source (which is the known MPS capable of generating the required narrow divergence photon beam) because the photons are delivered in a short pulse. A Cherenkov detector with a threshold just above $511 \mathrm{keV}$ could be a possible solution. If such detector with good detection efficiency could be developed, EZ-3D would be an even more powerful approach than dual energy for Z-discrimination using an MPS.

The simulations conducted confirm the expectation that significantly better $\mathrm{Z}$ discrimination can be achieved with a narrow energy spread beam, measuring transmission at several well defined energies, than with a bremsstrahlung beam that integrates transmission over the full energy range and may experience spectral change (beam hardening) when traversing the cargo. Narrow-energy-distribution, narrow-beam-divergence MPSs with the ability to select and rapidly switch energy over the full range of 1-10 MeV could therefore potentially enable the development of improved systems. These capabilities are characteristic of MPSs based on Thomson scattering. Energy switching could be important for Z-determination using the dual energy method. Resolving object overlap in highly cluttered cargos may further require at least two views or an EZ-3D type method, which is not addressed in detail here. 


\section{Raster Scanning and Dose Adaptation}

Existing broad energy-spread sources have cone beam emission geometry and require collimation to generate a fan beam used for one-dimensional scanning along the length of a container. The same methodology would be appropriate for nuclear reaction based MPSs. The small beam size and narrow divergence of Thomson sources preclude the fan beam approach, but these sources are ideal for raster scanning, assuming acceptable scan times can be achieved.

Raster scanning provides two important advantages: it can improve image quality as discussed above by reducing scattering contributions, and it enables 2D dose adaptation for each resolution element if the number of photons per shot can be controlled on a shot-by-shot basis. A simple scheme for dose adaptation across a cargo container may be to deliver one initial low-dose shot and then to follow it up with one or two shots at higher intensities. A faster technique may be to control the dose to the next pixel based on the knowledge of the transmitted signal for the previous pixel. As the raster scan proceeds to build up the image more spatial information may become available for better estimating the numbers of photons needed for the next pixel. Sharp changes in intensity value from low $\mathrm{Z}$ to high $\mathrm{Z}$ pixels may require more than one shot.

For raster scanning with a narrow-divergence photon beam, the dimensions of the container, the required scanning speed, and the achievable beam sizes define the repetition rate. The goal of scanning a container in $30 \mathrm{~s}$ is certainly ambitious but could be done in principle, with a $\sim 1 \mathrm{~cm}^{2}$ beam and 1 shot/location at $10 \mathrm{kHz}$. For dose modulation and dual energy measurement 2 or 3 shots per location may be required. Assuming a $1 \mathrm{~cm}$ beam diameter and a $20 \mathrm{kHz}$ repetition rate, a $40 \mathrm{ft}$ container could be scanned in one minute. By taking advantage of spot by spot dose modulation, an additional severalfold dose reduction could result from scanning the container with a pencil beam. Shorter scanning times or operation at lower repetition rates would be possible with larger beam diameters or adaptive beam diameters, using larger beams where highest spatial resolution or scatter rejection is not needed.

\section{II.1.4.2. Alarm Resolution and SNM Identification}

If a dense high- $Z$ object has been found and localized in a radiographic scan and determined to be suspicious, the object or location may need to be inspected in a secondary scan to either determine with high confidence that SNM is not present and to clear the alarm or to confirm the presence of nuclear materials. Active interrogation methods have been developed for this purpose including photofission and NRF. Secondary screening for SNM typically uses photofission, and use of energies below $10 \mathrm{MeV}$ is a priority. Initial calculations indicated that dose required to detect fissile material could be reduced by more than a factor of 10 because the entire MPS beam overlaps the photofission resonance, yielding much more efficient excitation compared to bremsstrahlung sources at $\sim 10 \mathrm{MeV}$ energies (where only a small fraction of photons are useful). Beyond photofission, highly specific NRF measurements are enabled at MPS energy spreads $\leq 2 \%$. NRF measurement times and doses could be lower by orders of magnitude compared to a bremsstrahlung source. Simulations were conducted to quantitatively assess capability for both signatures including realistic targets objects, beam transport and detection. In this report, photofission simulations are discussed in detail in Section II.2, Detection of Hidden SNM, and NRF simulations are discussed in detail in Section II.3, Treaty/Dismantlement Verification. Here, we apply the detailed results of those sections to assess their potential for alarm resolution and SNM identification (i.e., secondary or final screening) in cargo applications. 


\section{Photofission}

Active interrogation to induce detectable photofission signatures has been extensively studied, as discussed in the initial report $[7,73,74,75]$. Specifically, Passport Systems Inc. has recently demonstrated, using a bremsstrahlung source, that in cargo screening, prompt photofission neutrons are a strong indicator of fissionable material [61]. Passport's cargo screening system uses prompt neutron monitoring during the primary scan. In the analysis presented here we compare MPS use to conventional bremsstrahlung sources, assuming that in cargo screening the maximum photon energy is limited to less than $10 \mathrm{MeV}$ to comply with regulations for cargo radiation exposure. A monoenergetic photon source is attractive in this scenario because, in contrast to a bremsstrahlung source, all MPS photons have energies well above the fission threshold, resulting in much higher photofission efficiency.

To quantify MPS benefits for photofission screening, a test configuration was analyzed consisting of an HEU cube with a side length of $4.6 \mathrm{~cm}$ (i.e. close to $100 \mathrm{~cm}^{3}$ in volume and $2 \mathrm{~kg}$ in weight) positioned in the center of a cube with either $10,20 \mathrm{~cm}$ or $30 \mathrm{~cm}$ thick steel walls, which in turn was centered inside a cargo container. The photon source (i.e., the beam starting location) was $1.25 \mathrm{~m}$ from the outer wall of the container. Fission rates were calculated with MCNP for a $9 \mathrm{MeV}$ MPS and for bremsstrahlung source with a $9 \mathrm{MeV}$ endpoint energy and a $1 \mathrm{~cm} \mathrm{~W}$ beam hardening filter. For this comparison both beams were collimated to an angular divergence of $3.5 \mathrm{mrad}$ so that all photons would hit the HEU target. The fission rates per source photon are listed in Table 12 for steel wall thicknesses of $20 \mathrm{~cm}$ and $30 \mathrm{~cm}$. The number of fissions per source photon is $\sim 160 \times$ higher for the MPS than for the bremsstrahlung source due to a combination of less attenuation in the steel and higher photofission cross sections. For comparing the dose per fission imparted by the beams to an object in their path, one must take into account the different dose per photon for both beams. As seen in Table 9, the dose per photon to a water cylinder is $3.09 \times$ higher for the MPS than for the bremsstrahlung source, reflecting the higher average energy of the MPS photons. Thus, for the simulated cases, the MPS delivers a factor of 53 lower dose per fission than the bremsstrahlung beam. Such dose reduction would significantly benefit the use of photofission in the field.

Table 12: Fission rates per source photon in $2 \mathrm{~kg}$ HEU in steel boxes for MPS and bremsstrahlung beams.

\begin{tabular}{|l|l|l|}
\hline Wall thickness of steel box & $20 \mathrm{~cm}$ & $30 \mathrm{~cm}$ \\
\hline MPS 9 MeV & $5.18 \times 10^{-5}$ & $5.05 \times 10^{-6}$ \\
\hline Bremsstrahlung 9 MeV, $1 \mathrm{~cm}$ W filter & $3.19 \times 10^{-7}$ & $3.07 \times 10^{-8}$ \\
\hline Fission-rate ratio MPS/bremsstrahlung & 162 & 165 \\
\hline
\end{tabular}

Detailed simulations of photofission signal rates are described in section II.2, Detection of Hidden SNM. For energies below $10 \mathrm{MeV}$, the prompt neutron signature is strongest. The results can be extrapolated to the case considered here, as the configuration is similar, i.e., the same shielding thicknesses of $20 \mathrm{~cm}$ and $30 \mathrm{~cm}$ of steel. Although the HEU masses considered are very different, in both cases (the $2 \mathrm{~kg}$ HEU cube and the larger cylinder used as configuration A in Section II.2), the full photon beam intersects with the HEU object. For use of the prompt neutron signal the maximum allowed photon energy was set at $10 \mathrm{MeV}$ to avoid potential signature interference (see Section II.2) for single sided inspection, and the same limit applies to cargo container screening. A $9 \mathrm{MeV}$ MPS beam with a 10\% energy spread would be suitable. For the geometry and beam parameters given in Section II.2, the prompt neutron count rate was calculated to be $\sim 0.033$ counts per beam pulse $\left(6.9 \times 10^{8}\right.$ photons/pulse) for the $20 \mathrm{~cm}$ steel wall case. This result can be extrapolated to the cargo inspection case considered here. The case 
considered here has a $10 \times$ shorter HEU to detector distance $(2 \mathrm{~m})$ and a $4 \times$ reduced detector area $\left(1 \mathrm{~m}^{2}\right.$ instead of $\left.4 \mathrm{~m}^{2}\right)$, and hence the detected prompt neutron rate is increased $25 \times$. Correcting the fission rate for the differences in the beam energy spectrum $(9 \mathrm{MeV}$ with $10 \%$ energy spread vs. 6.7 MeV with $40 \%$ energy spread), and assuming $1 \times 10^{8} \mathrm{ph} /$ pulse on target, decreases the number of prompt neutrons by a factor of 1.7. Applying these factors yields a prompt neutron rate of 0.5 counts/pulse in the cargo screening case for $2 \mathrm{~kg}$ of HEU inside a steel cube with $20 \mathrm{~cm}$ thick walls. In a primary cargo scan with the parameters derived in the radiography section above, a $1 \mathrm{~cm}$ diameter beam and a single pulse per spot $\left(\sim 1 \mathrm{~cm}^{2}\right)$, the HEU cube would be hit by $\sim 20$ pulses, and 10 neutrons would be detected on average. While this is close to the statistical limit of detectability, even a few neutrons would likely cause an alarm because the neutron background from cosmic radiation is negligible during the short prompt neutron collection time, which is on the order of 1 us after each pulse. Increasing the detector area to a few $\mathrm{m}^{2}$ would increase statistics. This indicates that even during radiography scan with a MPS, SNM could be detected via photofission. Material presence or absence could then be easily confirmed by dwelling on the spot for, say, 1000 beam pulses.

For a steel wall thickness of $10 \mathrm{~cm}$, the prompt neutron rate would be $\sim 80$ counts/pulse or 1600 for a scan, and the HEU easily detected. For a steel wall thickness of $30 \mathrm{~cm}$ the prompt neutron rate would be $\sim 0.02$ counts/pulse, and several thousand pulses, i.e., a measurement time of several seconds at a $1 \mathrm{kHz}$ repetition rate, would be needed for detection. As indicated in Section II.2, composite shielding (e.g., borated polyethylene or "B-poly") and lead) of similar thickness can be penetrated using similar beam parameters.

The delayed gamma or neutron signals are generally weaker than the prompt neutron signal. The delayed gamma signature can be detected by pulsing the source on a macro scale, e.g., $0.5 \mathrm{~s}$ on and $0.5 \mathrm{~s}$ off, as described in section II.2. The detected net delayed gamma yields of section II. 2 can be extrapolated to the cargo screening case ( $2 \mathrm{~kg}$ HEU inside steel box with $20 \mathrm{~cm}$ thick walls) considered here. Assuming a $9 \mathrm{MeV}$ MPS beam, $1 \times 10^{8} \mathrm{ph} /$ pulse on target, $1 \mathrm{kHz}$ repetition rate, and a total detector size of $1 \mathrm{~m}^{2}$ at $2 \mathrm{~m}$ distance, it would take $\sim 1$ minute to exceed the critical decision level, which is much longer than for the prompt neutron signature. However, for very thick hydrogenous cargo with high neutron attenuation, the delayed gamma signal may exceed the prompt neutron counts and be the preferred signature.

A Thomson scattering source is particularly well suited for resolving an alarm caused by an object in an identified location because the low-divergence beam produces a high photon flux over a small area. In the analyzed example, a Thomson source operating at $9 \mathrm{MeV}$ and delivering $1 \cdot 10^{8}$ photons/shot on target at a $1 \mathrm{kHz}$ repetition rate would generate, when interrogating $2 \mathrm{~kg}$ of HEU behind $20 \mathrm{~cm}$ of steel, 500 prompt neutrons and 20 gamma-rays per $1 \mathrm{~m}^{2}$ detector area per second. This would enable detection with a high probability within a short interrogation time. Using higher repetition rate and/or longer inspection times, $2 \mathrm{~kg}$ of HEU could be detectable behind more than $30 \mathrm{~cm}$ of steel. Fissionable materials could be distinguished from fissile materials in several ways after an alarm has been triggered: passive gamma/neutron spectroscopy, differences in the time dependence of the delayed gamma emission, and possibly polarized photofission. Detection of the HEU via photofission could also be done with photons from a nuclear reaction based source. Because such sources emit into $4 \pi$, a very high source intensity is required induce a sufficient number of fissions. If the nuclear reaction source were at a distance of $2 \mathrm{~m}$ from the $2 \mathrm{~kg}$ HEU considered above, the solid angle subtended by the HEU would only be a $\sim 4 \times 10^{-5}$ fraction of $4 \pi$. For example, use of the $15.1 \mathrm{MeV}$ photons from the ${ }^{11} \mathrm{~B}(\mathrm{~d}, \mathrm{n} \gamma)^{12} \mathrm{C}$ has been suggested (this energy is higher than currently used for cargo). Taking into account that the fission yield is $\sim 6 \mathrm{x}$ higher at this energy than for $9 \mathrm{MeV}$ photons, the photon yield of the $4 \pi$ source would still need to be a few thousand times higher than that of a Thomson 
source to give the same delayed signature strength. Source photon energies above $10 \mathrm{MeV}$ are too high to exploit the prompt neutron signature, as further discussed in section II.2

\section{Nuclear Resonance Fluorescence}

NRF has been extensively studied and long been advocated for use in cargo screening because of its unique capability to identify specific isotopes and measure isotopic content. This specificity can potentially be utilized to detect and identify SNM, explosives and other contraband $[13,76]$. In the context of cargo screening, $\mathrm{NRF}$ is now seen as especially attractive for measuring the concentrations of light elements such as carbon and oxygen to provide cargo-screening systems with additional capabilities for detecting and identifying contraband. Key advantages of NRF over fission based approaches for SNM detection are the abilities to distinguish fissile material from fissionable material, and to perform isotopic assay. In the assessment of NRF for cargo screening, we considered NRF only for SNM detection, because contraband detection is outside the scope of this study. However, the scaling between bremsstrahlung and MPS performance is likely to be similar in both cases.

The main challenges of NRF are generally low NRF count rates due to small integrated resonance cross sections, and often a high background from scattered photons. Measurements with a bremsstrahlung source suffer from its broad energy spectrum. Only a small fraction of the photons are at resonance energy and contribute to the signal, while all others contribute to the background in the spectra and increase the radiation dose to the cargo. MPSs offer dramatic advantages for NRF measurements [20]. A key beam parameter for NRF is the spectral density, i.e., the number of photons per unit energy and time, at the resonance energy. For example, a Thomson scattering source generating $2 \times 10^{7} \mathrm{ph} /$ pulse with a $2 \%$ energy spread and operating at a $10 \mathrm{kHz}$ repetition rate could provide a spectral density of $5 \times 10^{6} \mathrm{ph} / \mathrm{eV} / \mathrm{second}$, which is roughly a factor of 100 higher than that of Passport's bremsstrahlung source operating with an end point energy of $3 \mathrm{MeV}$ and a $2.5 \mathrm{~mA}$ beam current. The higher NRF count rate in combination with a dramatically reduced scatter background can be expected to result in a greatly enhanced detection sensitivity together with orders of magnitude reduction in dose to the cargo [20].

For an initial estimate of the detection capability of an MPS-based NRF system, a simple test case was modeled using the MCNP radiation transport code. A small, $2 \mathrm{~cm}$ radius, $650 \mathrm{~g}$ HEU sphere is located in the center of a cargo container $(2.4 \mathrm{~m} \times 2.4 \mathrm{~m}$ cross section, $4 \mathrm{~mm}$ thick steel walls) that is filled with water at an average density of $0.6 \mathrm{~g} / \mathrm{cm}^{3}$. The MPS is assumed to deliver $3 \times 10^{7} \mathrm{ph} /$ pulse with a beam divergence of $0.2 \mathrm{mrad}$ onto the HEU sphere. Two different energy spread values, $2 \%$ and $0.2 \%$, were considered. The measurement times needed to achieve a 6 sigma confidence for detection of the HEU sphere were calculated for various configurations and beam parameters. The values in Table 13 assume the use of 24 HPGe detectors or a calorimetric detector around the witness foil and operation at $1 \mathrm{kHz}$ repetition rate.

Table 13: Measurement times for reaching 6-sigma confidence for detecting a 650 g HEU sphere when operating at $1 \mathrm{kHz}$ repetition rate.

\begin{tabular}{|c|c|c|}
\hline Measurement Method & $\begin{array}{c}\text { Energy spread }(\Delta \mathrm{E} / \mathrm{E}) \\
\text { FWHM }(\%)\end{array}$ & Measurement Time \\
\hline Backscatter & $0.2 \%$ & $100 \mathrm{sec}$ \\
\cline { 2 - 3 } 24 HPGe detectors & $2 \%$ & $17 \mathrm{~min}$ \\
\hline Transmission & $0.2 \%$ & $30 \mathrm{sec}$ \\
\cline { 2 - 3 } 24 HPGe detectors & $2 \%$ & $5 \mathrm{~min}$ \\
\hline Transmission & $0.2 \%$ & $30 \mathrm{sec}$ \\
\cline { 2 - 3 } Calorimetric detector & $2 \%$ & $55 \mathrm{~min}$ \\
\hline
\end{tabular}


When using HPGe detectors, the measurement times scale as expected with the spectral density of the beam, which accounts for the tenfold increase with $\Delta \mathrm{E} / \mathrm{E}$ increasing from $0.2 \%$ to $2 \%$. Identification of this small test object is possible in 5 minutes at $2 \%$ energy spread and in 30 seconds at $0.2 \%$ energy spread. The time needed for the transmission measurement with the calorimetric detector increases with the square of the energy spread because the signal to noise ratio decreases in addition to the $\mathrm{NRF}$ count rate with reduction in spectral density. Thus, a calorimetric detector requires a small energy spread, on the order of $0.2 \%$ or less. The NRF count rate scales with the MPS repetition rate so that for a $10 \mathrm{kHz}$ rate the measurement times are onetenth of those given in Table 13.

A second set of simulations was performed to assess NRF for the 2 kg HEU (93\% U-235) object (cube with side length of $4.6 \mathrm{~cm}$, density of $18.6 \mathrm{~g} / \mathrm{cm}^{3}$ ) used in the assessments of cargo radiography and photofission. As shown in Figure 15, the HEU is centered inside a steel box with $20 \mathrm{~cm}$ inside wall length and $10 \mathrm{~cm}$ thick walls. The steel box itself is centered within a cargo container modeled as a box with $5 \mathrm{~mm}$ thick Fe walls and $240 \mathrm{~cm}$ side length. Simulated were both backscatter and transmission measurements. The backscatter detector is located $145 \mathrm{~cm}$ from the center of the cargo at an angle of 164 degrees relative to the beam centroid direction. For the transmission measurement the U-235 Witness Foil or WF (also known as the scatter target) is $1 \mathrm{~cm}$ thick and $2 \mathrm{~cm}$ in diameter and positioned $330 \mathrm{~cm}$ down-beam from the back end of the cargo container. This distance is unimportant as long as the WF diameter is larger than the beam diameter. The transmission detector is positioned $100 \mathrm{~cm}$ from the WF at an angle of 135 degrees relative to the beam centroid direction.

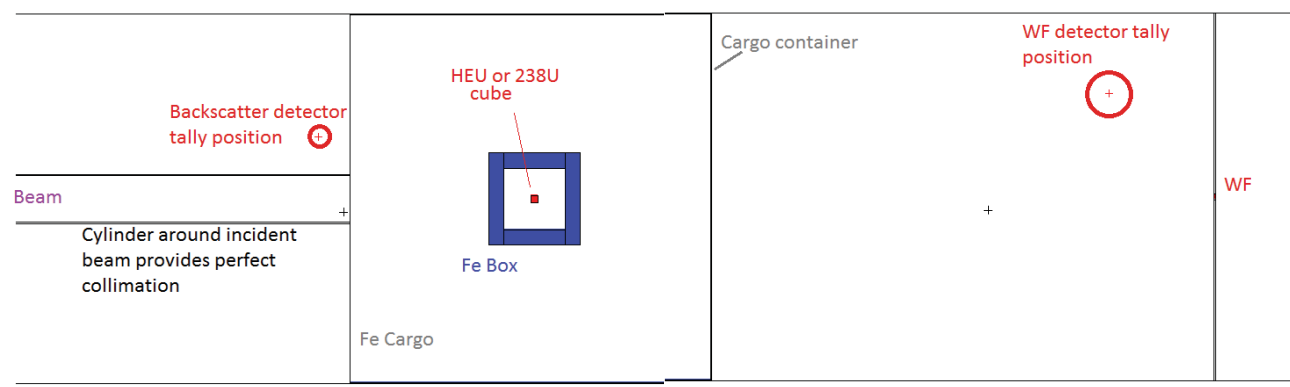

Figure 15. Simulated setup for determining NRF signal rates and measurement times. The backscatter detector is located $145 \mathrm{~cm}$ from the center of the cargo at an angle of 164 degrees with respect to the beam axis and perfectly shielded from scatter from the container wall. For the transmission measurement the scatter target is positioned $330 \mathrm{~cm}$ down-beam from the back end of the cargo container. The transmission detector is positioned $100 \mathrm{~cm}$ from the scatter target at an angle of $135^{\circ}$ relative to the beam axis.

Simulations were conducted for Resonant (Res) and Non-Resonant (NR) photons separately for determining signal and background rates using a beam with an $8 \mathrm{eV}$ energy spread, the width of the Doppler-broadened resonance, centered on the U-235 resonance at $1.7336 \mathrm{MeV}$. In the resonant simulation, NRF and other forms of scattered photons were tallied at the transmission detector and the backscatter detector positions. In the non-resonant simulations, photonuclear physics were turned off to provide the elastic scatter background for the NRF signal-to-noise ratio calculation and the flux due to the non-resonant component of source photon scattering. In the third simulation the HEU target in the cargo container was replaced with pure U-238, which has identical attenuation properties for non-resonant photons, but the simulation allows the scatter target in the transmission setup to undergo NRF. An integrated cross section of $36 \mathrm{eV} \cdot \mathrm{b}$ [8] was used in the simulations for the U-235 NRF resonance; it should be noted that in a second experiment Wang et al. measured $22 \mathrm{eV} \cdot \mathrm{b}$ [77]. The results presented here would need to be scaled to apply the lower value. 
Detector response simulations were performed separately. It is assumed that the photon flux is spatially uniform over the front surface of the detector (area $60 \mathrm{~cm}^{2}$ ). Simulations of energy deposition probabilities were conducted for an unshielded HPGe detector, and for the same detector positioned behind low energy photon filters of $0.5,0.75$ and 1.0 inches of $\mathrm{Pb}$ to reduce undesired low-energy photons. Detector full energy deposition probabilities are used to convert MCNP flux tallies to full-energy counting probabilities. Energy-dependent incident detector flux tally spectra are convolved with the detector response to ascertain total count rate expectations.

The resonant and non-resonant simulation results are combined to determine expected system performances for beams with 1.5 and $15 \mathrm{keV} \mathrm{rms}$ wide energy spreads corresponding to $0.2 \%$ and $2 \% \Delta \mathrm{E} / \mathrm{E}$ FWHM [17]. The method assumes that the photon physics does not change over this energy range and is the same as that of the non-resonant 8-eV-wide beam, so that the proportions of non-resonance and resonance photons can be scaled by the relative fraction of each within the beam distribution and convolved with detector responses. This directly gives a total-spectrum count rate. The NRF-signal strength relative to background is established by comparing the scaled NRF strength to the elastic (coherent) scatter strength produced in the nonresonant simulations across the energy resolution of the detector system (assumed here to be 3 $\mathrm{keV}$ for HPGe detectors). Calculated flux distributions to which detector response functions were applied are indicated in Figure 16 for the resonant simulation (blue), the non-resonant simulation (red). Also shown are calculations of the background for the NRF peak resulting from nonresonant scatter. In the case of a $0.2 \% \Delta \mathrm{E} / \mathrm{E}$ beam, the non-resonant flux (red) was scaled by the fraction of a Gaussian distribution that is within $\pm 4 \mathrm{eV}$ of the centroid. The resulting non-resonant background is shown as cyan. Lastly, to represent the non-resonant flux due to a $2 \% \Delta \mathrm{E} / \mathrm{E}$ beam, the down-scattered portion of the simulated flux was scaled, while the simulated elastic scatter flux was additionally distributed across a profile representative of the beam, as shown in black. These simulations were conducted with $5 \mathrm{keV}$ energy spacing, which somewhat under-predicts the intensity of the NRF peak, relative to HPGe detector resolution. The resonant signal is visible above the nonresonant signal in both cases.
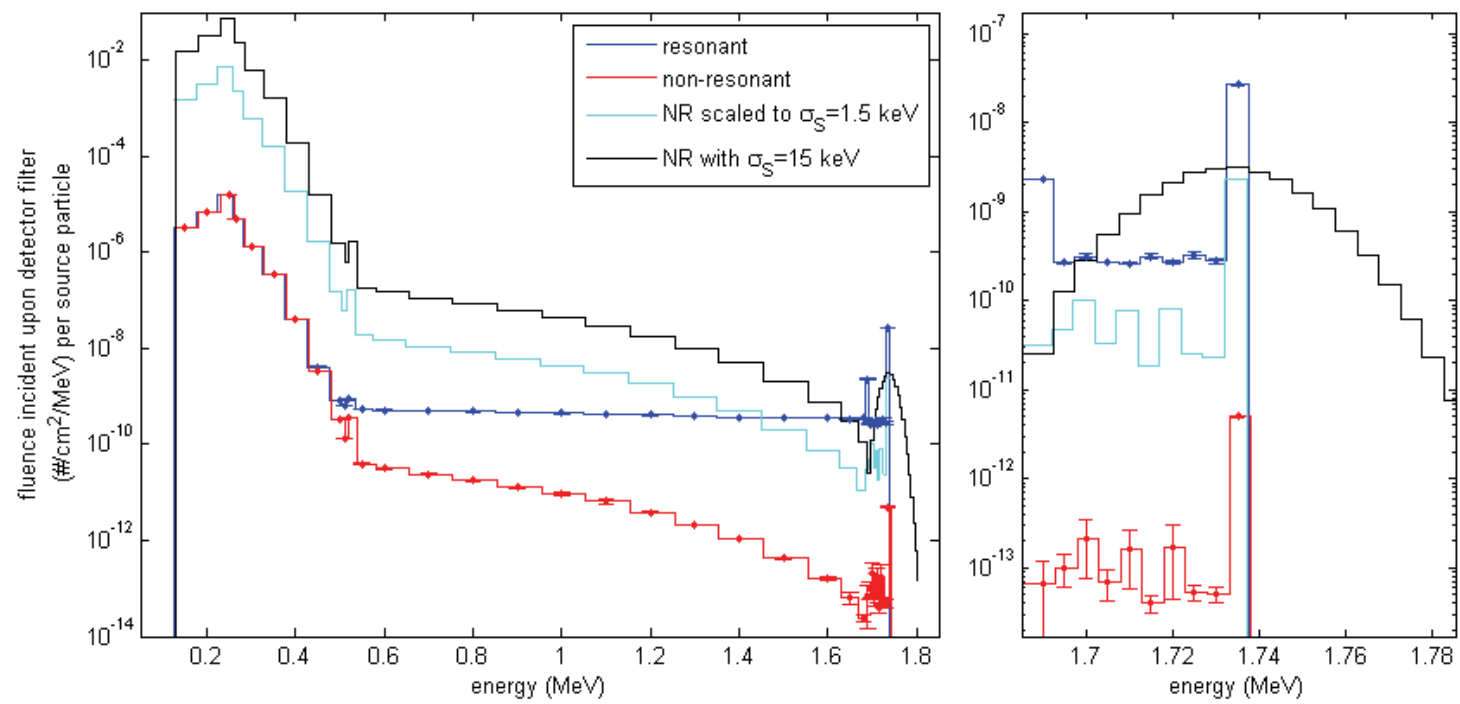

Figure 16. (on left) Simulated spectra incident upon the backscatter detector for the configuration indicated in Figure 15. The resonant (blue) and non-resonant (red) spectra are directly obtained from MCNP simulations of photon sources with widths of $8 \mathrm{eV}$ incident upon a HEU target inside shielding and a cargo container with and without NRF physics, respectively. The simulation's statistical errors are indicated, although often smaller than the widths of the lines. The non-resonant scatter produces background for the NRF peak, and these effects are approximated for the $0.2 \%$ and $2.0 \%$ beams as cyan and black, respectively. (on right) Same data, zoomed in on the beam-energy region. 
The backscatter geometry NRF count probability, total count probability and the signal-tonoise ratio are listed in Table 14 for 1.5 and $15 \mathrm{keV} \mathrm{rms}$ width beams and for HPGe detectors behind $0.5,0.75$ and 1 inch of $\mathrm{Pb}$. For the 0.5 " $\mathrm{Pb}$ filter, the count rate was high enough that it is the limiting factor. Hence, for this case the beam intensity per shot (source photons per shot) was reduced from the nominal maximum of $3 \times 10^{7}$. The estimated time to make a measurement to confirm presence or absence of U-235 at a level of 6 sigma is based on the number of needed NRF counts for a given signal-to-background ratio $\xi$, which is calculated as $36 / \operatorname{sqrt}(1+2 / \xi)$, i.e., for large $\xi, 36 \mathrm{NRF}$ counts correspond to a 6-sigma measurement. The shortest measurement times in Table 14 were obtained for the 0.75 " filter cases. For the cases considered the measurement times scale with the energy spread of the beam. The times given Table 14 are for a single HPGe detector and a $1 \mathrm{kHz}$ source repetition rate. Assuming the use of 24 detectors as in Table 13 and a source repetition rate of $10 \mathrm{kHz}$, the measurement times reduce to $4 \mathrm{~s}$ and $40 \mathrm{~s}$ for photon beam energy spreads of $0.2 \%$ and $2 \%$, respectively. Measurement times scale with the attenuation of the beam in cargo and shielding and the attenuation of the resonant photons from the HEU into the detector.

Table 14: Simulation results for backscatter NRF measurements on a $2 \mathrm{kG}$ HEU object in a $10 \mathrm{~cm}$ thick steel box, centered in a container. Calculated measurement times are for one detector and for a MPS with $\leq$ $3 \times 10^{7}$ photons per shot at a $1 \mathrm{kHz}$ repetition rate.

\begin{tabular}{|c|c|c|c|c|c|c|c|c|c|c|}
\hline 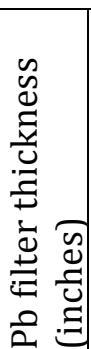 & 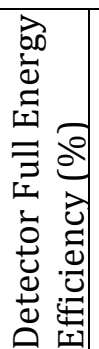 & 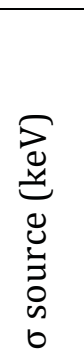 & 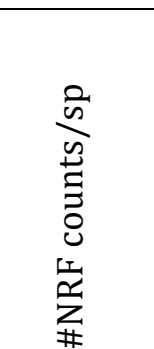 & 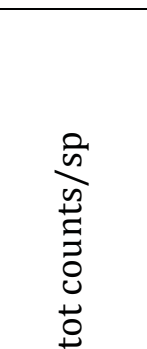 & 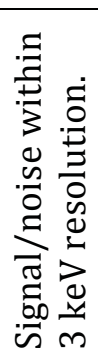 & 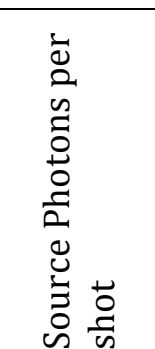 & $\begin{array}{l}\frac{\overrightarrow{0}}{0} \\
\frac{1}{n} \\
\underline{n} \\
\vdots \\
\vdots \\
0\end{array}$ & 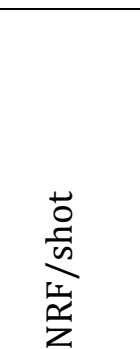 & 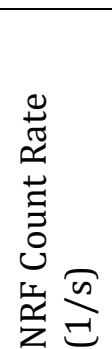 & 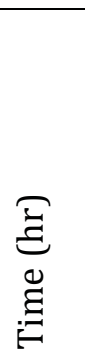 \\
\hline 0.5 & 12.5 & 1.5 & $2.1 \mathrm{E}-12$ & $3.65 \mathrm{E}-8$ & 8.3 & $5.5 \mathrm{E}+06$ & 0.2 & $1.15 \mathrm{E}-5$ & 0.0115 & 0.97 \\
\hline 0.5 & 12.5 & 15.0 & $2.1 \mathrm{E}-13$ & $3.64 \mathrm{E}-8$ & 1.5 & $5.5 E+06$ & 0.2 & $1.15 \mathrm{E}-6$ & 0.0012 & 9.8 \\
\hline 0.75 & 8.7 & 1.5 & $1.47 \mathrm{E}-12$ & $3.36 \mathrm{E}-9$ & 8.3 & $3.0 \mathrm{E}+07$ & 0.10 & $4.41 \mathrm{E}-5$ & 0.0441 & 0.25 \\
\hline 0.75 & 8.7 & 15.0 & $1.47 \mathrm{E}-13$ & $3.33 \mathrm{E}-9$ & 1.5 & $3.0 \mathrm{E}+07$ & 0.10 & $4.41 \mathrm{E}-6$ & 0.0044 & 2.6 \\
\hline 1 & 6.2 & 1.5 & $1.05 \mathrm{E}-12$ & $5.26 \mathrm{E}-10$ & 8.3 & $3.0 \mathrm{E}+07$ & 0.02 & $3.15 \mathrm{E}-5$ & 0.0315 & 0.35 \\
\hline 1 & 6.2 & 15.0 & $1.05 \mathrm{E}-13$ & $5.03 \mathrm{E}-10$ & 1.5 & $3.0 \mathrm{E}+07$ & 0.02 & $3.15 E-6$ & 0.0031 & 3.6 \\
\hline
\end{tabular}

For the simulation of transmission measurements the resonant and non-resonant simulation results were combined to determine expected system performances for beams with 1.5 and 15 $\mathrm{keV}$ rms energy spreads in a similar way as in the backscatter simulations. The simulated spectra are shown in Figure 17. In the case under consideration here, $2 \mathrm{~kg}$ of HEU inside a box with $10 \mathrm{~cm}$ thick walls, the attenuation of resonant photons is too strong to measure the quantity of U-235 within the target area in a reasonable time. However, the objective in cargo screening is simply the detection of HEU if present. Thus the measurement time of interest is the time it takes to collect the number of NRF counts that are needed to determine with the required statistical probability the absence of resonant absorption [15]. Assuming one HPGe detector (as in the backscatter simulations), $3 \times 10^{7}$ photons/pulse, and $1 \mathrm{kHz}$ source repetition rate, the 6-sigma measurement times for $0.2 \%$ and $2 \% \Delta \mathrm{E} / \mathrm{E}$ FWHM beams are $5.3 \mathrm{hr}$ and $155 \mathrm{hr}$, respectively. Scaling this to 24 detectors and a $10 \mathrm{kHz}$ repetition rate as for the backscatter measurement, the measurement times are $80 \mathrm{~s}$ and $2300 \mathrm{~s}$, much larger than for the backscatter method. For a $1 \mathrm{~cm}$ thick HEU plate (instead of $4.6 \mathrm{~cm}$ ) the corresponding estimated measurement times drop to $3 \mathrm{~s}$ and $80 \mathrm{~s}$ showing that the transmission method becomes competitive with the backscatter 
technique for thin HEU objects. The transmission method also benefits more rapidly as MPS energy spread reduces, and may also be particularly attractive if MPS energy spreads below $0.2 \%$ become realistic. In such cases, it has been proposed that calorimetric detectors could be used, simplifying measurement [10].
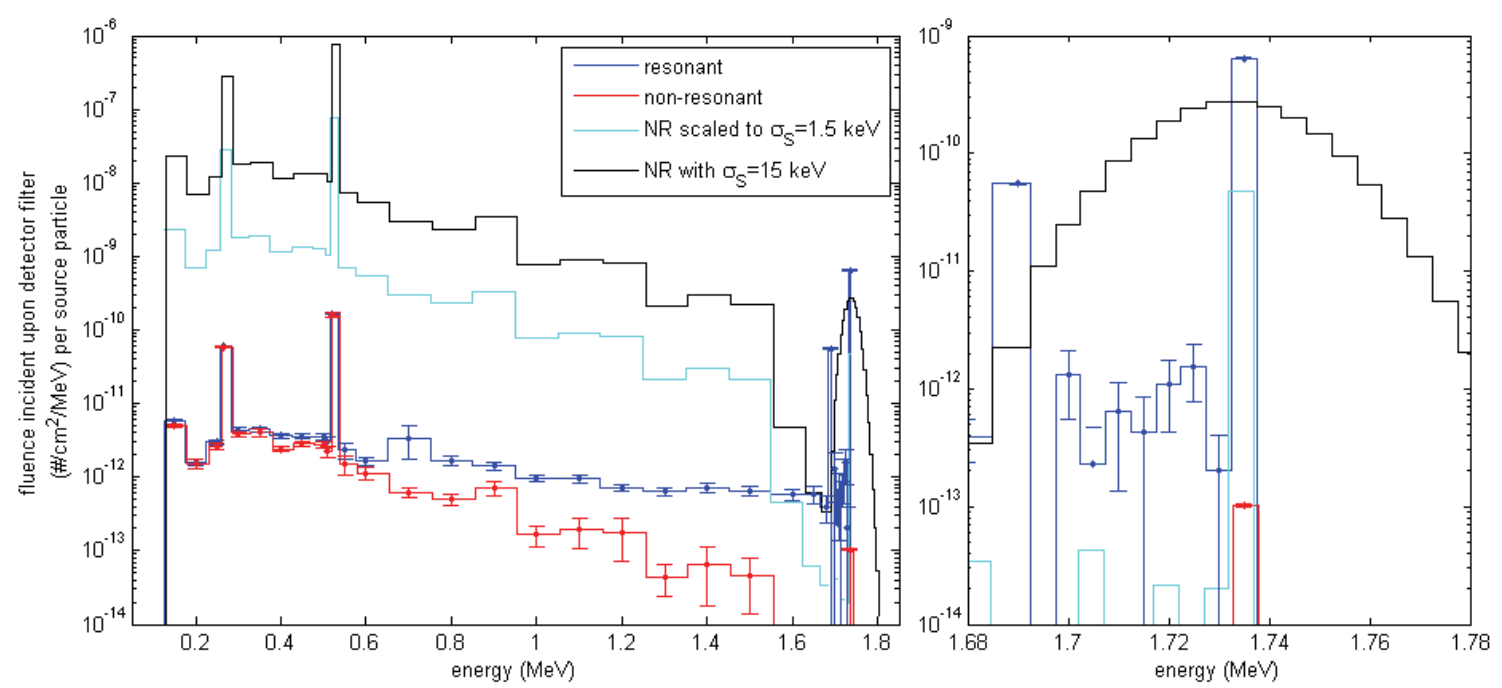

Figure 17. On left: Simulated spectra incident upon the transmission detector for the transmission configuration indicated in Figure 15. The resonant (blue) and non-resonant (red) spectra are directly obtained from MCNP simulations of photon sources with widths of $8 \mathrm{eV}$ incident upon a U-238 target inside shielding and a cargo container with and without NRF physics, respectively. The simulation's statistical errors are indicated, although often smaller than the widths of the lines. The non-resonant scatter produces background for the NRF peak, and these effects are approximated for the $0.2 \%$ and $2.0 \%$ beams as cyan and black, respectively. On right: Same data, zoomed in on the beam-energy region.

Energy spreads of $2 \%$ and repetition rates of $10 \mathrm{kHz}$ are realistic goals for Thomson sources and would make it possible to detect $2 \mathrm{~kg} H E U$ inside $10 \mathrm{~cm}$ thick steel shielding, or within containers filled with water, in tens of seconds in a backscatter measurement with HPGe detectors. Further in the future, Thomson sources with an energy spread of a fraction of a percent may become available; the results indicate such a source would greatly increase capability.

\section{II.1.5. Impact of and Requirements for MPS Systems}

\section{Radiography}

Significant potential benefit was indicated for radiography applications using monoenergetic photon beams at energies ranging from 3-9 MeV and with typical fluxes in the range of $10^{10}$ photons per second for initial capabilities to $10^{12}$ photons per second for high performance applications. Energy spreads of approximately 10-20\% are desirable. Pulsed sources should have repetition rates in the $\mathrm{kHz}$ range to enable initial capabilities (corresponding to $10^{7}$ to $10^{8}$ photons per shot), while repetition rates in the $10-50 \mathrm{kHz}$ range would enable high performance. Several MPS capabilities are important, each of which delivers benefits, which compound. For the configurations evaluated, replacing bremsstrahlung with a monoenergetic source having similar emission angle and pulse structure enables reductions in radiography dose by factors of 3-4× due to the narrow energy spread alone. All comparisons reported here assume that the MPS or bremsstrahlung source itself is well-shielded and does not contribute to the dose. Contrast in $\mathrm{Z}$ is also increased severalfold for the assessed configurations by use of dual-energy MPS ratios. This 
can enable effective discrimination in cases that are not currently accessible, and/or reduce the dose required to achieve discrimination in cases that are currently accessible by severalfold. Sources that produce beams with small divergence in particular could be of great value to cargo imaging applications by producing a small diameter beam at the target (i.e., a pencil beam). This configuration mitigates contrast degradation due to scatter and hence reduces the required dose. The additional reduction in dose, both to stowaways and (due to scatter from cargo) to surroundings, could be a factor of a few on top of the reduction due to the monoenergetic energy spectrum depending on cargo and system design. Small divergence is more effective in mitigating scattering than control of the photon energy spread. Hence MPSs with narrower angle emission than bremsstrahlung fans will provide the strongest advantage. Correspondingly, MPS systems with broader emission angles than bremsstrahlung fans may not improve dose or image quality in cases where scattering is strong. By taking advantage of spot-by-spot dose modulation, an additional severalfold dose reduction could result from scanning the container with a pencil beam. In contrast to scanning with a fan beam, the dose to empty or weakly attenuating regions above and below highly attenuating material could in many cases be reduced by at least an order of magnitude.

In pencil beam scanning, the spatial resolution can be defined by the beam diameter and not by the size of the detector element. Hence the pencil beam also removes effects of scattering within the detector, which is often significant [5], further compounding the contrast advantage and dose reduction to be obtained. This also raises the possibility that the imaging detector could be much simpler with larger, higher-detection-efficiency elements, which could further reduce dose. In-detector scatter would not be an issue as signals from neighboring elements could be added. Evaluation of this effect was beyond the scope of the current study.

The divergence requirement for use of a pencil beam is set by the desired scanning resolution, since the beam divergence and the source-to-cargo distance define beam size at the container wall. For container radiography applications, a beam size of $1 \mathrm{~cm}$ at the container wall can be achieved at a distance of $5 \mathrm{~m}$ using a beam divergence of $2 \mathrm{mrad}$, or at $2.5 \mathrm{~m}$ using $4 \mathrm{mrad}$. Using bremsstrahlung or nuclear reaction sources, such collimator angles would discard almost all of the available photons. Thomson sources, on the other hand, intrinsically generate beams with $\sim \operatorname{mrad}$ divergence at energies of a few $\mathrm{MeV}$ and energy spreads of $10-30 \%$.

For Screening and Interdiction of Shielded SNM, systems in use or under consideration include small buildings (such as the Passport system at the Port of Boston). As MPSs are currently being developed to fit smaller form factors, such an installation may be a logical first step for field applications. Systems at or below the size of a small truck, with correspondingly modest electrical requirements (at the $60 \mathrm{kWe}$ level), would also be highly desirable.

Combining the effects of monoenergetic transmission, increased $\mathrm{Z}$ contrast, scattering mitigation, and dose adaptation, the simulations indicate that a narrow-divergence MPS could enable superior resolution combined with typical dose reductions of one to two orders of magnitude (target and configuration dependent). Experimental validations of MPS advantages for radiography and $\mathrm{Z}$ determination, conducted by other groups during the span of this project $[34,44]$, have confirmed that simulations accurately predict MPS advantages for the cases tested by the respective experiments (which were somewhat less dense than those simulated here). This increases confidence in the predicted MPS advantages reported here for screening relevant cases.

\section{Alarm Resolution}

Monoenergetic photon sources strongly benefit secondary screening and alarm resolution by increasing the fraction of the source photons that overlap the cross-section of interest. Photofission excitation is less efficient in cargo applications than in single sided applications 
because of the limitation in a cargo context to photon energies less than $10 \mathrm{MeV}$. Nonetheless, photofission signatures are stronger than NRF for realistic MPS parameters, and for photons below $10 \mathrm{MeV}$ the prompt neutron signature is strongest. Additionally, photofission places less stringent demands on the MPS, with energy spreads of $10 \%$ being desirable and (as described in Section II.2, Detection of Hidden SNM) energy spreads up to $40 \%$ being usable. A source capable of generating such a beam near $9 \mathrm{MeV}$ is important, prioritizing energy adjustability.

For both photofission and NRF, narrow source angular divergence is needed to overlap the beam with the object of interest. For the $\sim 2 \mathrm{~kg}$ objects considered here, a beam divergence of $<$ $10 \mathrm{mrad}$ is ideal. With a source at $9 \mathrm{MeV}$ producing $\sim 10^{8}$ photons/shot into $3 \mathrm{mrad}$ opening angle, photofission-based detection of $2 \mathrm{~kg}$ HEU mass behind $10 \mathrm{~cm}$ steel shielding could be accomplished during a radiography scan with no change in rate. A few thousand pulses (seconds at $1 \mathrm{kHz}$ ) could identify the same quantity behind $30 \mathrm{~cm}$ of steel. NRF is more suitable where composition analysis is needed, either isotopic composition of SNM or contraband detection. It places more stringent demands on the MPS. At energy spreads of $2 \%$ and repetition rates of 10 $\mathrm{kHz}, 2 \mathrm{~kg}$ of HEU could be detected inside $10 \mathrm{~cm}$ thick steel shielding, or within containers filled with water, in tens of seconds. Sources with an energy spread of a fraction of a percent would greatly increase NRF capability. 


\section{II.2. Detection of Hidden SNM}

\section{II.2.1. Introduction}

This application section considers a constrained detection scenario; i.e., it assumes that a general search location has been pre-determined and the volume to be probed is constrained to a few cubic meters. Inspection scenario examples include a car, small truck, small shed, or a location inside a house. While potentially similar in several aspects, the current application is considered categorically separate from fixed-site, commercial cargo screening, since, in the present case, physical access limitations and other characteristics of a particular scenario may exclude the use of traditional radiography or transmission NRF (e.g., access may be available only from one side). The objective in this single-sided inspection application is to confirm either the presence or absence of nuclear material. For clearing an object, sufficient information about the amount of shielding surrounding the nuclear material must be available to firmly conclude that a lack of observed fission signatures is not due to shielding but proves the absence of SNM. Allowable inspection times may be longer than those defined for cargo screening, since interruption of commerce may be less of a concern, and source and detector distances relative to the target may be extended and varied; measurement times in the range of minutes, and distances from a few meters to tens of meters, are reasonable to expect.

Photofission was identified as the technique using MPSs that could enable a capability for detection of hidden SNM. The most compelling advantage of an MPS-based system, when compared with bremsstrahlung-based systems, is the potential for a marked increase in the induced fission rate per unit dose on target. The continuous energy distribution of a bremsstrahlung beam results in a large fraction of the beam's photons having energies that lie outside the giant dipole resonance. In contrast, a selectable-energy, narrow-bandwidth photon source can produce an inspecting beam in which essentially all incident photons have energies that can readily induce fission. Similarly, the spatial beam profiles of some MPSs are more narrowly divergent than that of a traditional bremsstrahlung source. This permits significantly higher photon density incident on the target SNM and hence higher signal. The beam from a bremsstrahlung source can be heavily collimated to produce a small angular divergence, comparable with a MPS. However, by clipping away most of the beam, such an effort would severely reduce the number of fission-inducing photons on target per original source photon, resulting in a lower photofission rate.

This section briefly reviews the current status of hidden SNM detection via photofission, followed by a high-level summary of the decision criteria and observable signals. The results of an initial assessment are briefly discussed before higher-fidelity calculations and modeling results to evaluate photofission techniques are presented. Detailed simulations were performed, quantifying capability for various target and shielding scenarios. It is worth noting that depending on the concept of operations, it may be possible to perform Compton backscattering, or similar techniques, in single-sided cases to narrow the inspection area. For example, a dense object identified inside a vehicle would then be scanned. A pulsed Thomson source may enable such backscatter imaging that, possibly in combination with other methods, can be used to determine or put a limit on the extent of shielding. A more thorough assessment of backscatter imaging was beyond the scope of this project.

\section{II.2.2. Background and Current Status}

Substantial research has been reported in the literature demonstrating the utility of photofission-based active inspection methodologies for detecting the presence of nuclear material using bremsstrahlung sources. Procter et al. demonstrated the ability to differentiate 
between depleted uranium and several non-fissionable targets, even when shielded by wood, water, or lead [75]. Their methodology, which combines delayed gamma-ray and neutron measurements, focused on $\sim 2 \mathrm{~m}^{3}$ container volumes, with promising results for scaling to larger inspection volumes. Increased backgrounds and possible interfering signals were identified as potential issues for the technique's applicability to implementation outside the laboratory environment [75]. Carrel et al. presented experimental measurements using high-energy delayed gamma rays from photofission [78]. While the focus of that work was characterizing nuclear waste packages, the techniques described could inform other applications where shielding or matrix materials are likely to be encountered. Stevenson et al. describes a tiered inspection process in which radiography measurements are leveraged to localize an area of interest inside a container [79]. Their technology is intended for commercial cargo screening and the localization via radiography may be unavailable in many general search scenarios. Nevertheless, the demonstration of both the delayed gamma ray and the prompt fission neutron signatures is directly applicable to the current effort.

While much of the reported research relies on bremsstrahlung-based inspection beams to generate the fission signatures of interest, methods using monoenergetic photon sources have also been reported $[80,20,81]$, although such methods are less common than their bremsstrahlung counterparts. General signatures and dose tradeoffs for MPSs and comparison to bremsstrahlung sources indicate that MPSs should operate in the 10-20 MeV range. Goldberg et al. present a concept for a dual-use accelerator, from which monoenergetic photons are produced via proton and deuteron-induced reactions [80]. The source described is intended to support screening of massive cargo, and the photon energies being sought could be used to induce photofission where nuclear material is present. Nevertheless, the article focuses on the source itself and does not include further discussion regarding signatures or detection. Similarly, the FIGAR0 technique relies on 6-7 MeV gamma rays resulting from proton reactions on fluorine [81]. Photon energies in that range would induce photofission and other photoneutron reactions of interest. It is unclear at this stage whether such a source would be able to provide an acceptable level of flux on a target area to be useful in a realistic search application. Use of a tunable-energy MPS allows detailed characterization and use of photofission signatures. The HIGS fixed facility Thomson source has been used for such studies including investigation of possible photofission resonances for discrimination between types of SNM [15].

\section{Signatures and Criteria}

In the context of a general search application, the criterion for detection was chosen to be the observation of photofission signals, above natural and/or induced backgrounds, from which the presence (or absence) of nuclear material could be verified. More specifically, a decision of "Detected" or "Not Detected" is made here based on whether the net detected yield surpassed a critical decision level as defined by L.A. Currie for a false positive probability of $1 \%$ [82]. For this work, we assumed, as explained below, that source beam parameters can be chosen such that any source-induced background is negligible. Currie's critical decision level then simplifies to include a lone term, which accounts for the presence of passive background. Values for passive gamma ray and neutron backgrounds were taken from Ref. [7].

The signatures of interest for this application are based on the neutron and gamma-ray emissions inherent to fission reactions:

- Delayed Neutrons. Ideally, a fission signature based on delayed neutrons would include all neutrons that are emitted following $\beta$ decay of excited fission fragments. However, source-induced prompt radiation may interact in the environment and continue to impinge on the detectors for some time following the source pulse. Previous research has 
shown that a careful selection of a "delayed region" essentially eliminates any sourceinduced neutron backgrounds from contributing to the delayed neutron signal [7]. For the current effort, total delayed neutron detection rates were adjusted by assuming the delayed region excluded the time period of 0-13.8 $\mathrm{ms}$ following an inspection pulse. Depending on the inspection system and environment for a given scenario, the specific delayed region will likely vary; however, the above value was selected for the current calculations to be consistent with recent research presented in the literature [7].

- Delayed Gamma Rays. Gamma rays emitted following $\beta$ decay of excited fission fragments can also provide a unique signature for the presence of nuclear material $[83,84]$. As with delayed neutrons, techniques relying on delayed gamma rays must account for sourceinduced prompt signals that interact in the environment and contribute to the detected signals at times following the source pulse. Again, a "delayed region" is defined to minimize contributions of prompt signals to the delayed gamma-ray signature. In the current work, gamma-ray emission rates were adjusted by excluding the time period of 0$30.3 \mathrm{~ms}$ following an inspection pulse. A delay of $30.3 \mathrm{~ms}$ was chosen for consistency and qualitative comparisons with recent research [7]. In practice, the specific value would be based on the employed inspection system and surrounding environment. In contrast to delayed neutrons, low-energy gamma rays are probable from many different reactions even when relatively low inspection source energies are used, and are emitted on timescales similar to those characteristic of fission-produced delayed gamma rays. To mitigate this contribution, the delayed gamma-ray signature is typically defined as highenergy ( $>\sim 3 \mathrm{MeV}$ ) gamma rays detected within the delayed time region. Previous work has shown that, by keeping inspection photon energies below $\sim 20 \mathrm{MeV}$, almost all of the source-induced, delayed gamma-ray background can be mitigated [7].

- Prompt Neutrons. The prompt neutron signature relies on neutrons emitted from fission fragments shortly after scission has occurred $\left(10^{-15} \mathrm{~s}\right)$ [74]. The large amount of energy released during fission allows for prompt neutron energies to exceed $\sim 10 \mathrm{MeV}$, regardless of the energy of the neutron or photon that induced fission. Prompt neutrons are also significantly more abundant during fission than are delayed neutrons, although potential interferences to this signature occur at much lower inspection beam energies. To compensate for such interferences, photon energies are typically limited to $10-12 \mathrm{MeV}$, allowing for an energy threshold to be set on the detected signal. The threshold value depends on both the inspection source energy and the neutron separation energies for isotopes comprising natural and/or commonly encountered materials. Neglecting beryllium, ${ }^{207} \mathrm{~Pb}$ has one of the lowest $(\gamma, \mathrm{n})$ thresholds of commonly encountered isotopes at $6.7 \mathrm{MeV}$. Hence, assuming that the maximum incident photon energy is $10 \mathrm{MeV}$, setting a threshold value of $3.3 \mathrm{MeV}$ minimizes contributions of non-fissionable materials to the prompt neutron signature. At photon energies above $8.84 \mathrm{MeV}$ neutrons from the breakup of deuterium have energies above the $3.3 \mathrm{MeV}$ threshold, but they usually are not a significant source of interference because of the low natural abundance of deuterium.

\section{II.2.3. Potential Capabilities and Advantages of MPS-based System - Initial Assessment}

The initial assessment considered a general search scenario: a $26.75 \mathrm{~kg}$ (containing $25.0 \mathrm{~kg}$ 235U) HEU sphere located in the center of a container whose walls are composed of increasing thicknesses of water, steel, or a composite containing borated polyethylene and lead. Wood was also considered as a potential container wall material. Because the results were consistent with the water case, the wood-based calculations are not explicitly included here. Time to detection at fixed distance, and the distance at which detection can be accomplished in fixed time, were 
evaluated for each shielding setup. These initial calculations are not precise, but allowed evaluation of parameter ranges that were used to set up detailed calculations of cases where detection is realistic.

For preliminary assessments, simplified calculations were performed for the uranium sphere, centrally located, inside air-filled shielding boxes that each had interior dimensions of $4 \times 4 \times 4$ feet. The walls of the boxes were 1, 6, or 12-inch thicknesses of water or steel, or a composite consisting of 4 inches of borated polyethylene and 2 inches of lead. To further simplify the comparisons between a bremsstrahlung source and a narrow-bandwidth source, each was initially assumed to produce $2 \times 10^{8}$ photons per pulse, which is representative of realistic systems, and to have a repetition rate of $15 \mathrm{~Hz}$. In reality, repetition rates in the range of one to tens of $\mathrm{kHz}$ are reasonable to expect for planned MPSs. In that case, the time between pulses would be insufficient to make use of the delayed neutron or delayed gamma-ray signatures; interlaced operation (many pulses during an irradiation period, followed by a counting period with the source shut off) would be required for an inspection to take advantage of the delayed signatures. Optimization of the interlaced operation parameters was not included in the initial assessment, but was part of the detailed simulation study described in the next section. For the bremsstrahlung beam case, a $19 \mathrm{MeV}$ endpoint energy was selected as a rough optimum for detection. While induced fission rates will correspondingly increase with bremsstrahlung endpoint energies, endpoints above $\sim 20 \mathrm{MeV}$ are expected to produce non-negligible sourceinduced backgrounds [7]. The MPS calculations were performed for a $30 \%$ energy spread (FWHM), centered at $14 \mathrm{MeV}$, which is the energy where overlap with the resonance is optimized. These parameters were adjusted for the prompt neutron signature assessments, using a 20\% beam energy spread centered at $9.75 \mathrm{MeV}$.

Recognizing that maximum source energy and counting windows can be readily selected such that any source-induced background can be neglected, total fission rate as a function of dose on target was chosen as an initial metric to illustrate the potential advantage of employing a MPSbased system versus a conventional bremsstrahlung source. With a distance from the source location to the center of the target sphere set at $4 \mathrm{~m}$, fission rates as a function of dose on target were calculated for the three wall thicknesses of each material. For comparisons between the two source types, the starting beam diameter was the same for both sources $(3.8 \mathrm{~cm})$. As noted above, this beam diameter should not be interpreted as a high-precision approximation of either source, but rather was chosen to allow for direct comparison of the two sources' induced fission production as a function of dose at the target location. At a $4 \mathrm{~m}$ distance the target fully subtends either beam; hence, the number of target atoms in the beam was also consistent between the sources.

In the case of 1-inch water shielding, the calculations suggested that the MPS beam would cause $\sim 3.9 \times$ more induced fission reactions per rem on target, compared to the bremsstrahlung beam. For the 6 -inch water shielding, the MPS generated $\sim 3.5 \times$ more fission reactions per rem on target than the bremsstrahlung beam; 12 inches of water shielding lowered this to $\sim 3.1 \times$. Replacing the water shielding with wood produced very similar results. The ratio of MPS to the bremsstrahlung source for induced fission per rem on target was slightly lower for the same thicknesses of steel shielding. Nevertheless, the lowest value of that ratio was still 2.4. The ratio calculated for the composite case was 3.5, similar to the 6-inch water and 1-inch steel cases. Specific values for each of the cases are shown in Table 15. As noted above, MPSs with intense, narrowly collimated beams may allow additional dose reduction if the beam diameter on target is comparable to or smaller than the size of the SNM. For distances considered here, this corresponds to divergence at the milliradian level. 
Table 15: Ratio of MPS vs. bremsstrahlung induced fissions/rem

\begin{tabular}{|c|c|c|}
\hline Material & Thickness (inches) & MPS/Bremsstrahlung \\
\hline \multirow{3}{*}{ Water } & 1 & 3.9 \\
\hline & 6 & 3.5 \\
\hline & 12 & 3.1 \\
\hline \multirow{3}{*}{ Steel } & 1 & 3.6 \\
\hline & 6 & 2.8 \\
\hline & 12 & 2.4 \\
\hline Composite & $\begin{array}{c}\text { (Borated Poly) } \\
2 \text { (Lead) }\end{array}$ & 3.5 \\
\hline
\end{tabular}

Potential capabilities of a MPS-based system were examined for each signature using the HEU sphere and shielding boxes described above. The operational parameters of the MPS were consistent with those described above for the photofission signatures, and critical decision levels, $\mathrm{L}_{c}$, were established for a false positive probability of $1 \%(\alpha=0.01)$. These $\mathrm{L}_{c}$ 's were based on Ref. [82] and assumed that the background is well-defined such that the standard deviation of the net yield is dominated by the standard deviation of the gross yield (signal plus background). In the examined scenarios, the detectors were located with the source, at an initial distance of $20 \mathrm{~m}$ from the target. The calculations were focused on the measurement time required for the detected yield to exceed the corresponding critical decision level, with a $600 \mathrm{~s}$ maximum time allowed. Approximate distances at which the object may be detected in a set measurement time of $600 \mathrm{~s}$ were also calculated for each case.

Passive neutron and background yields were taken from Ref. [7], total detector surface area was set at $4 \mathrm{~m}^{2}$, and the intrinsic detection efficiencies were assumed to be $4 \%$ for delayed neutrons and 30\% for prompt neutrons and delayed gamma-rays $\left(\mathrm{E}_{\gamma}>3 \mathrm{MeV}\right)$. Delayed neutron and gamma-ray yields were based on the number of induced fission reactions as calculated from MCNP6 simulations. Delayed neutrons and high-energy delayed gamma rays were assumed to be emitted at a rate of 0.014 and 0.14 per fission reaction, respectively [84,85]. The number of delayed neutrons leaving the shielding and available for detection was calculated based on an average energy of $450 \mathrm{keV}$; the same calculation for the delayed gamma-rays was based on a 4 $\mathrm{MeV}$ average energy. The number of prompt neutrons $\left(\mathrm{E}_{\mathrm{n}} \geq 3.3 \mathrm{MeV}\right)$ available for detection relied on the LLNL fission model [86].

Tables 16-18 highlight the results of the calculations for each of the signatures, assuming a beam repetition rate of $15 \mathrm{~Hz}$. Along with the shield material and wall thickness, results are given for the total measurement time required for the detected yield to surpass the critical decision level, given a source/detector to target distance of $20 \mathrm{~m}$. In several cases, calculations suggested the corresponding yields would fail to reach $\mathrm{L}_{\mathrm{c}}$ within $600 \mathrm{~s}$; hence, the distance at which the detected yields would be expected to exceed the decision level for a $600 \mathrm{~s}$ measurement time are also included. It should be noted that these are approximate values provided as a preliminary assessment and for comparison. The follow-on simulation study (see next section) provided higher fidelity.

The calculated results suggest the object of interest would be detectable, via one or more of the three signatures, at a $20 \mathrm{~m}$ source/detector distance within $600 \mathrm{~s}$ for each of the shield materials with the exception of the 12 -inch steel case. For the later, all three signatures failed to produce a yield above the respective $L_{c}$ for a measurement time $\leq 600 \mathrm{~s}$, with a $15 \mathrm{~Hz}$ source repetition rate. At 12 inches of steel, the incident photon beam is attenuated such that the number of induced fission reactions was too low to produce a sufficient yield. However, by decreasing the source/detector to target distance to 12 meters, the delayed neutron signature could be used. The 
prompt neutron signatures could be used to $\sim 2 \mathrm{~m}$, while the distance required for the delayed gamma-ray signature with 12-inch steel shielding was calculated to be very short - less than $1 \mathrm{~m}$. The maximum distance $(152 \mathrm{~m})$ was calculated for the prompt neutron signature, with the object shielded by 1 inch of water. As the water thickness is increased, the potential distances for the prompt neutron signature fall below the corresponding distances for the delayed gamma-ray signature in these initial results, owing to increasing neutron moderation in the water shielding. It is important to note that a very conservative background assumption was made for these prompt neutron estimates, i.e., the background was integrated over the full measurement time. As discussed in the simulation study section below, the prompt neutrons are counted for only very short time periods after each micro-pulse so that the signal-to-background ratio can be greatly improved by gating the detector accordingly. This can dramatically increase the sensitivity of the prompt neutron signature making it the strongest of all three signatures.

Table 16: Time required for detected delayed neutron yield to exceed the critical decision level and approximate maximum distances at which the delayed neutron yield would exceed $\mathrm{L}_{\mathrm{c}}$ for a $600 \mathrm{~s}$ measurement.

\begin{tabular}{|c|c|c|c|}
\hline $\begin{array}{c}\text { Shield } \\
\text { Material }\end{array}$ & Thickness (inches) & $\begin{array}{c}\text { Time Required (s) @ } \\
\mathbf{2 0 ~} \mathbf{~ m}\end{array}$ & $\begin{array}{c}\text { Approximate Distance } \\
\text { (m) to Detect in 600 s }\end{array}$ \\
\hline \multirow{3}{*}{ Water } & 1 & $<1$ & 98 \\
\cline { 2 - 4 } & 6 & 2 & 52 \\
\cline { 2 - 4 } & 12 & $>600$ & 10 \\
\hline \multirow{3}{*}{ Steel } & 1 & $<1$ & 88 \\
\cline { 2 - 4 } & 6 & 7 & 44 \\
\cline { 2 - 4 } & 12 & $>600$ & 12 \\
\hline \multirow{2}{*}{ Composite } & $\begin{array}{c}4 \text { (Borated Poly) } \\
2 \text { (Lead) }\end{array}$ & $>600$ & 7 \\
\hline
\end{tabular}

Table 17: Time required for detected delayed gamma-ray yield to exceed the critical decision level and approximate maximum distances at which the delayed gamma-ray yield would exceed $\mathrm{L}_{\mathrm{c}}$ for a $600 \mathrm{~s}$ measurement.

\begin{tabular}{|c|c|c|c|}
\hline $\begin{array}{c}\text { Shield } \\
\text { Material }\end{array}$ & Thickness (in) & $\begin{array}{c}\text { Measurement Time } \\
\text { Required (s) @ 20 } \mathbf{~ m}\end{array}$ & $\begin{array}{c}\text { Approximate Distance } \\
\text { (m) to Detect in 600 s }\end{array}$ \\
\hline \multirow{3}{*}{ Water } & 1 & $<1$ & 131 \\
\cline { 2 - 4 } & 6 & $<1$ & 121 \\
\cline { 2 - 4 } & 12 & $<1$ & 105 \\
\hline \multirow{3}{*}{ Steel } & 1 & $<1$ & 110 \\
\cline { 2 - 4 } & 6 & 269 & 24 \\
\hline \multirow{2}{*}{ Composite } & $\begin{array}{c}\text { (B) } \\
\text { 2 (Lead) }\end{array}$ & $>600$ & $<1$ \\
\hline
\end{tabular}


Table 18: Time required for detected prompt neutron yield to exceed the critical decision level and approximate maximum distances at which the prompt neutron yield would exceed $\mathrm{L}_{\mathrm{c}}$ for a $600 \mathrm{~s}$ measurement.

\begin{tabular}{|c|c|c|c|}
\hline $\begin{array}{c}\text { Shield } \\
\text { Material }\end{array}$ & Thickness (in) & $\begin{array}{c}\text { Measurement Time } \\
\text { Required (s) @ 20 } \mathbf{~ m}\end{array}$ & $\begin{array}{c}\text { Approximate Distance } \\
(\mathbf{m}) \text { to Detect in 600 } \mathbf{~}\end{array}$ \\
\hline \multirow{3}{*}{ Water } & 1 & $<1$ & 152 \\
\cline { 2 - 4 } & 6 & $<1$ & 105 \\
\cline { 2 - 4 } & 12 & $<1$ & 63 \\
\hline \multirow{3}{*}{ Steel } & 1 & $<1$ & 138 \\
\cline { 2 - 4 } & 6 & 8 & 44 \\
\hline \multirow{2}{*}{ Composite } & $\begin{array}{c}12 \\
\text { (Borated Poly) } \\
2 \text { (Lead) }\end{array}$ & $>600$ & 2 \\
\hline
\end{tabular}

It is important to note that the above calculations used a source repetition rate of $15 \mathrm{~Hz}$, and assumed the beam was incident on the object for the entirety of an inspection. In reality, it is expected that an inspection would scan a container or vessel with much greater surface area, or volume, than the object of interest. Further, repetition rates $10^{3}-10^{4}$ times higher are anticipated in the near term (few years) for planned MPSs. To begin to address this, additional calculations were performed for the same object and containers used above, but considering a total inspection time of $600 \mathrm{~s}$ to inspect the entire container. Based on the cross-sectional area of the object, $\sim 151$ individual scans of $4 \mathrm{~s}$ each could be performed in a $600 \mathrm{~s}$ inspection time, for which at least one would have the beam incident on the object of interest (using an initial beam divergence of 3.5 mrad). For this assessment, interlaced operation was approximated with a macropulse repetition rate of $0.5 \mathrm{~Hz}$; each macropulse was $1 \mathrm{~s}$ long and consisted of micropulses with a repetition rate of $10 \mathrm{kHz}$. The distance from the source and detectors to the center of the inspection object was set at $20 \mathrm{~m}$.

The results of this initial assessment indicated that detection is realistic through 12 inches of shielding composed of any of the evaluated materials at distances up to approximately $12 \mathrm{~m}$ using a $\sim 20 \%$ energy spread and $\sim 3 \times 10^{12}$ photons/second delivered into a few-mrad divergence angle. The narrow divergence angle motivates use of a Thomson-type source, which would likely need to operate at a repetition rate of $\sim 10 \mathrm{kHz}$ to deliver sufficient flux. This assessment excluded further evaluation of detection at very long standoff (100 m or greater). Based on the initial results, the more detailed and higher fidelity simulation study reported on in the following section was conducted to more accurately evaluate capability and MPS parameter requirements.

\section{II.2.4. Assessment of MPS Performance: Simulations}

\section{Problem Definition and Setup}

The simulation study for this application area assessed the ability of an MPS-based system to confirm the presence of nuclear material and the information this data can give for determination of absence. Based on initial calculations, the material is housed inside a container of meter-scale size and shielded with varying thicknesses of water, steel, or composite material (lead and borated polyethylene). Assessing the thickness of shielding through which signals would be observed were material present gives information on the size of object within which a determination of absence could be made. 
A representative geometric layout of the simulations is shown in Figure 18. The inspection objects were placed in the center of a $7.7 \mathrm{~m}^{3}$ box ( $2 \mathrm{~mm}$ stainless steel walls), with a source-totarget distance of $20 \mathrm{~m}$. The detectors were assumed to be co-located with the source and had a total surface area of $4 \mathrm{~m}^{2}$. Since the focus of the study was potential advantages and capabilities afforded by an MPS-based system, rather than on the performance or development of detector technologies, subsequent calculations assumed that the intrinsic efficiency of the detectors was $30 \%$ for prompt neutrons and high-energy delayed gamma-rays, and $4 \%$ for delayed neutrons.

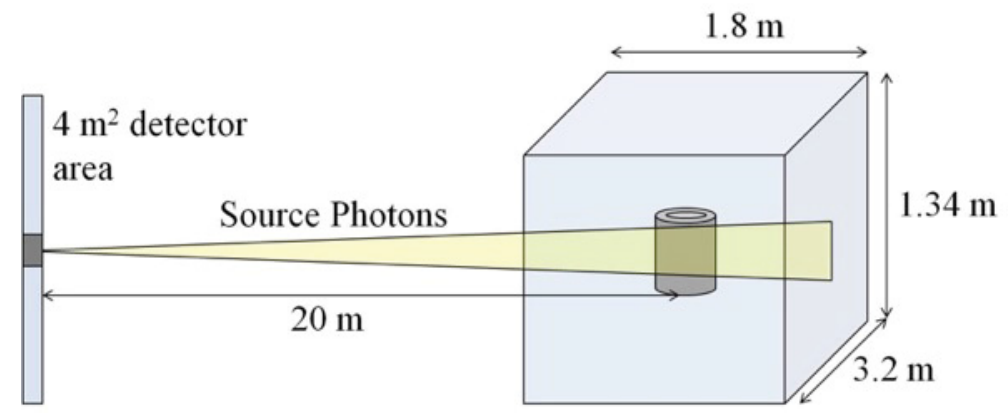

Figure 18. Representative setup used for the simulations.

The inspection object was based on HEU storage castings used at Y-12 that have been presented in the literature [87]. These HEU annuli comprise $\sim 18 \mathrm{~kg}$ of $93.2 \%$ enriched uranium, with an outer diameter of 5 inches. For the simulations, the inner diameter was set to 3 inches; the height was set at $11.3 \mathrm{~cm}$ to match the reported mass of the annuli. Three inspection object configurations were considered:

- Configuration A: single cylinder, axis orthogonal to the beam (Figure 19A)

- Configuration B: single cylinder, axis centered with the beam (Figure 19B)

- Configuration C: two cylinders, axes parallel to the beam (Figure 19C)

The ability to detect the presence of fissionable material was then assessed using these inspection object configurations and the following shielding conditions:

- No shielding present

- Stainless steel

○ $5,10,20$ or $30 \mathrm{~cm}$ of steel shielding

- Water

○ $5,10,20$ or $30 \mathrm{~cm}$ of water shielding

- Composite

○ $5 \mathrm{~cm}$ of $\mathrm{Pb}+25 \mathrm{~cm}$ borated polyethylene shielding

○ $10 \mathrm{~cm}$ of $\mathrm{Pb}+20 \mathrm{~cm}$ borated polyethylene shielding

- $20 \mathrm{~cm}$ of $\mathrm{Pb}+10 \mathrm{~cm}$ borated polyethylene shielding

Detailed simulations were conducted using the objects and shielding setup defined above. Appropriate MPS beam parameters for effective stimulation of prompt and delayed signatures were first evaluated. Thresholds for the evaluated signatures were evaluated in greater detail. Simulations were then conducted using high-fidelity descriptions of the object(s) of interest, containers/shielding, and the environment. 


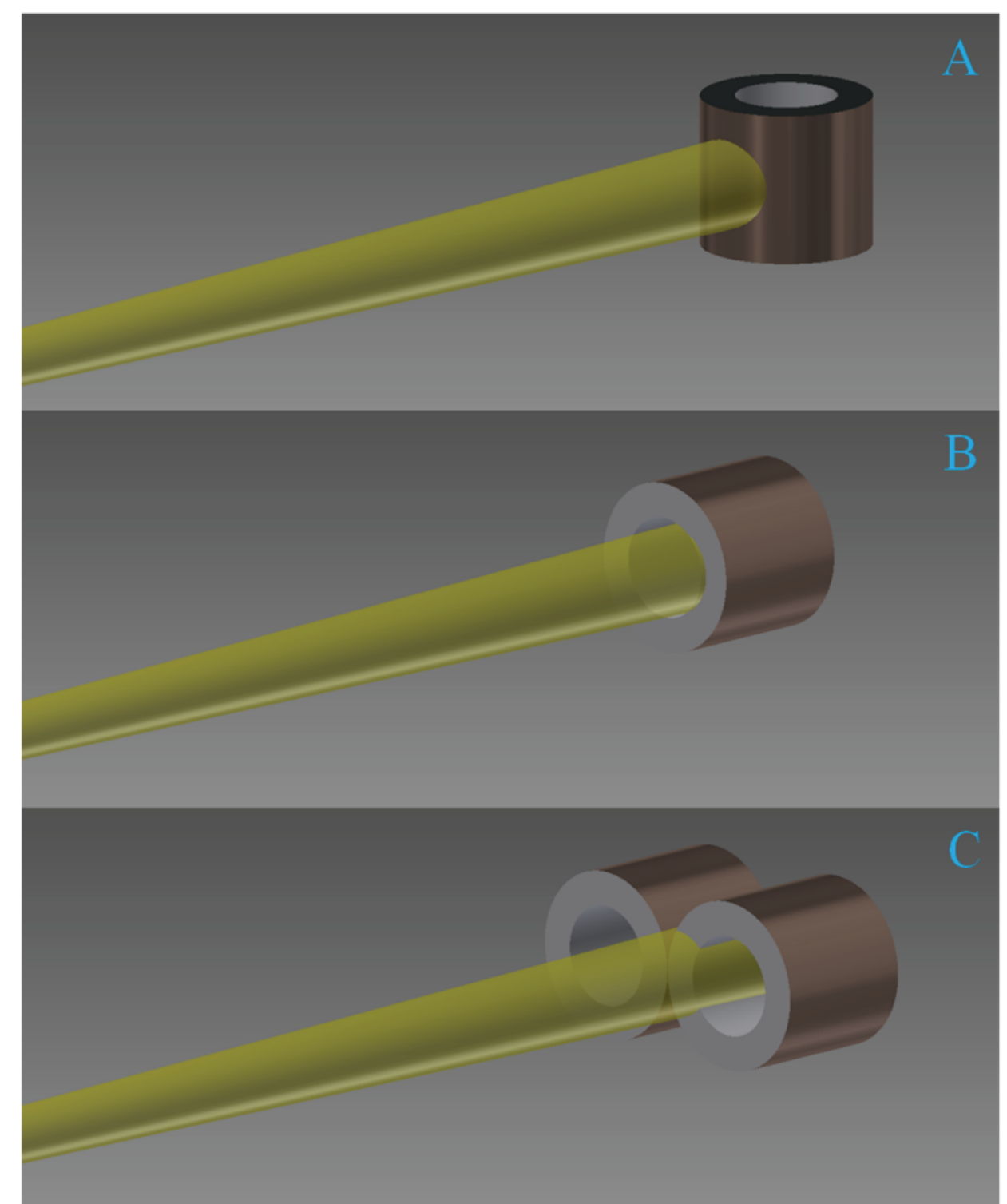

Figure 19. Inspection object configurations considered for the simulations. Each cylinder is $11.3 \mathrm{~cm}$ in height, with inner and outer diameters of 3 and 5 inches, respectively. Each consists of $\sim 18 \mathrm{~kg}$ of 93.2\% enriched uranium, and is based on the HEU storage castings presented in [87].

\section{MPS Parameter Optimization to Maximize Fission Signatures}

In order to fully assess an MPS-based system's potential capability, the optimal beam parameters were first investigated. Because this application depends on concentration of flux on a relatively small object (the whole box subtends only $10 \mathrm{mSR}$ ), a narrow-divergence source, such as a Thomson source, is a priority. For such a source, the dependence of the fission signatures on photon beam energy and energy spread were characterized including realistic tradeoffs between photon source energy spread and yield. Temporal pulsing structure was then evaluated using software developed to tally induced fission as a function of time.

For a Thomson-type MPS, the number of source photons emitted per shot was assumed to increase linearly with increasing width of the energy distribution as is realistic [88]. This 
substantially affects optimization. Narrow energy spread results in having few photons available. On the other hand, broad energy spread may mean that photons in the high-energy tail of the distribution have energies high enough to be problematic. The appropriate energy spread and energy are a compromise between these limits. The energy distribution was approximated by a Gaussian distribution down to the $\sim 2 \%$ level, at which point the photon flux was assumed to be zero, which emulates the physical cutoff due to the maximum electron energy:

$$
\begin{gathered}
\Phi(E)=0 \text { if } \frac{(E-b)^{2}}{2 c^{2}} \geq 4 \\
\Phi(E)=\frac{1}{c \sqrt{2 \pi}} e^{\frac{-(E-b)^{2}}{2 c^{2}}} \text { otherwise. }
\end{gathered}
$$

In these equations, $\mathrm{b}$ is the beam's centroid energy, and $\mathrm{c}=\mathrm{FWHM} / 2.3548$. The values used here are based on an extrapolation from a linear fit to anticipated photon numbers. Then, with these considerations, the expected fission production in an arbitrary target can be calculated for a range of beam centroid energies and energy widths. The total fission production rate per source pulse is calculated from:

$$
P=\int_{E_{i}}^{E_{f}} N(c) \frac{d \Phi(E)}{d E} \sigma(E) d E,
$$

where $N(c)$ is the number of photons per pulse, $d \Phi(E) / d E$ is the photon flux in the target, and $\sigma(\mathrm{E})$ is the photofission cross section.

Previous research available in the literature (e.g., [7]) has shown that, for incident photon energies below $\sim 20 \mathrm{MeV}$, the measured yield of high-energy delayed gamma rays or delayed neutrons from non-fissionable targets is consistent with passive background levels. An exception for high-energy gamma rays was observed from a beryllium target; however, beryllium is potentially a material of interest and, therefore, would not necessarily constitute a false positive. Hence, when optimizing the beam's centroid and percent energy spread, the calculations considered only those energy distributions that were below $20 \mathrm{MeV}$. Figure 20 presents the induced photofission yield versus beam centroid energy and percent energy spread. These results suggest an incident beam energy of $13.1 \pm 25 \% \mathrm{MeV}\left(8.5 \times 10^{8}\right.$ photons per source pulse $)$ as optimal for producing fission and generating the high-energy delayed gamma-ray and delayed neutron signatures in an arbitrary ${ }^{235} \mathrm{U}$ target without generating problematic non-negligible source-induced background.

For generating the prompt neutron signature described above, the beam parameters that were found to be optimal for maximizing the delayed signatures would produce excessive prompt neutrons above threshold from non-fissionable materials having low neutron separation energies. For example, as part of the selected scenarios that were simulated for this application, composite shielding comprised of varying thicknesses of lead and borated polyethylene was considered. For these cases, the relatively low $(\gamma, n)$ thresholds of the naturally occurring lead isotopes allow for a significant yield of neutrons above $3.3 \mathrm{MeV}$. Hence, when optimizing the beam parameters for the prompt neutron signature, the maximum allowed incident photon energy was set at $10 \mathrm{MeV}$. Figure 21 shows the induced photofission yield versus beam centroid energy and percent energy spread. In this case, the calculations suggest an incident beam energy of $6.7 \pm 20.5 \% \mathrm{MeV}$ $\left(6.9 \times 10^{8}\right.$ photons per source pulse $)$ is optimal for producing the prompt neutron signature while also minimizing potential signature interferences. 


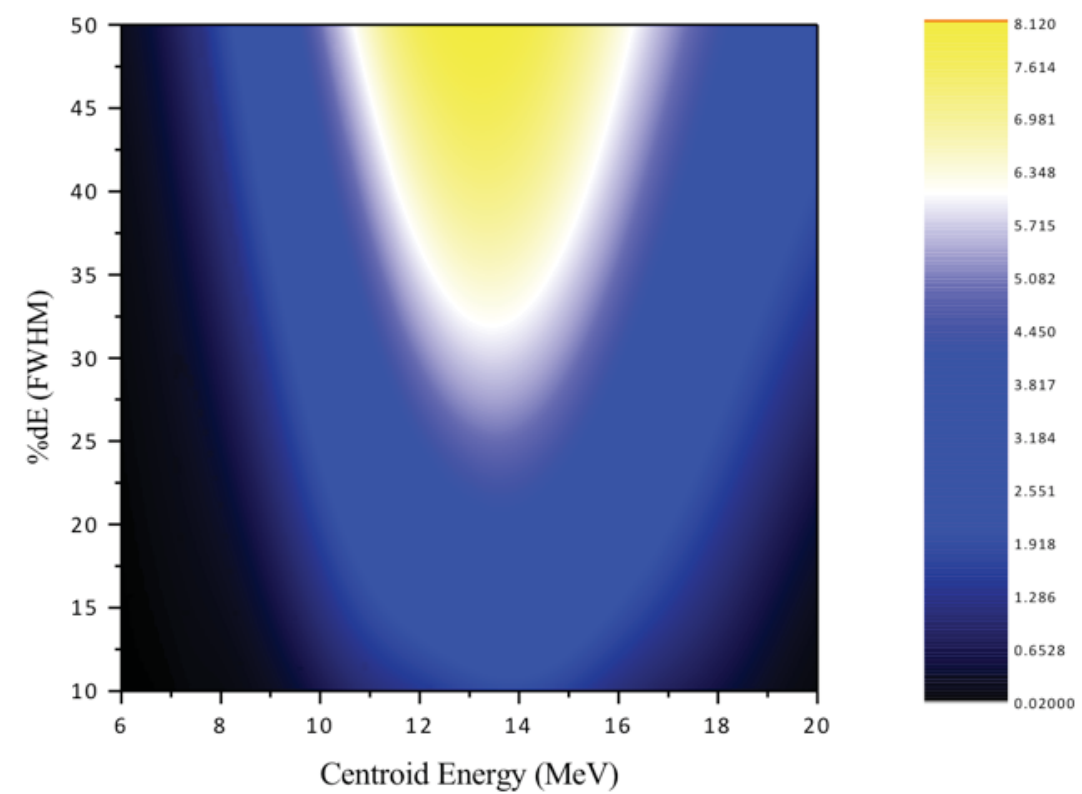

Figure 20. Relative induced photofission yield versus beam centroid energy and energy spread up to $20 \mathrm{MeV}$ maximum photon energy.

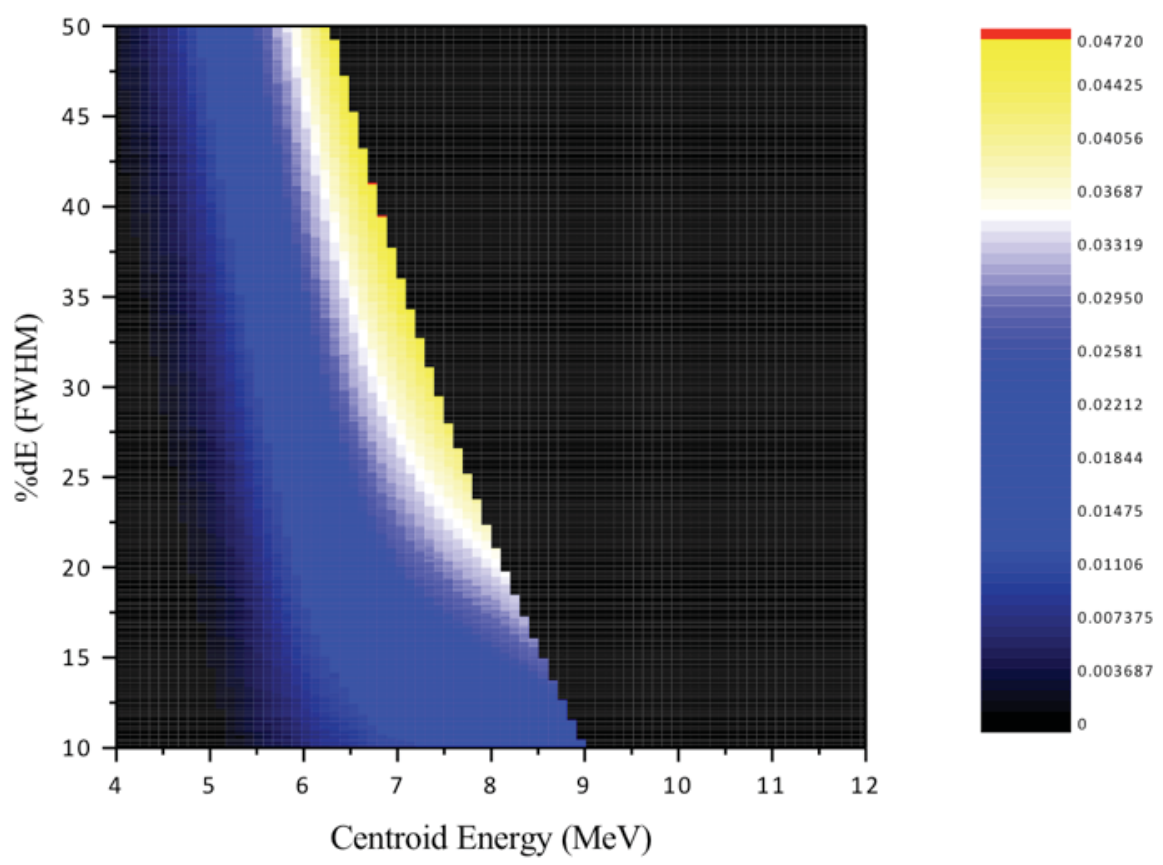

Figure 21. Relative induced photofission yield versus beam centroid energy and energy spread, up to 9 MeV maximum photon energy.

The final aspect of source parameter optimization was an assessment of the beam pulsing structure. For the target-to-detector distances considered here $(20 \mathrm{~m})$, pulsing the source with a repetition rate of $1 \mathrm{kHz}$ provides sufficient time $(1 \mathrm{~ms})$ for prompt neutrons produced during a given source pulse to impinge upon the detectors prior to the next successive source pulse. However, the need to exclude prompt regions when relying on delayed gamma-ray or neutron signatures requires modifications to the beam pulsing structure. In other words, the delayed signatures would not be reliable with a source operated continuously at $1 \mathrm{kHz}$. For these signatures, an interlaced pulsing mode (i.e., pulse at $1 \mathrm{kHz}$ during an "On" period, then completely 
shut off during an "Off" period) is needed. An example of an interlaced pulsing structure is shown in Figure 22. The Off period occurs during the second phase of each macropulse, and represents the time during which gamma rays or delayed neutrons would be detected (excluding a short prompt region).

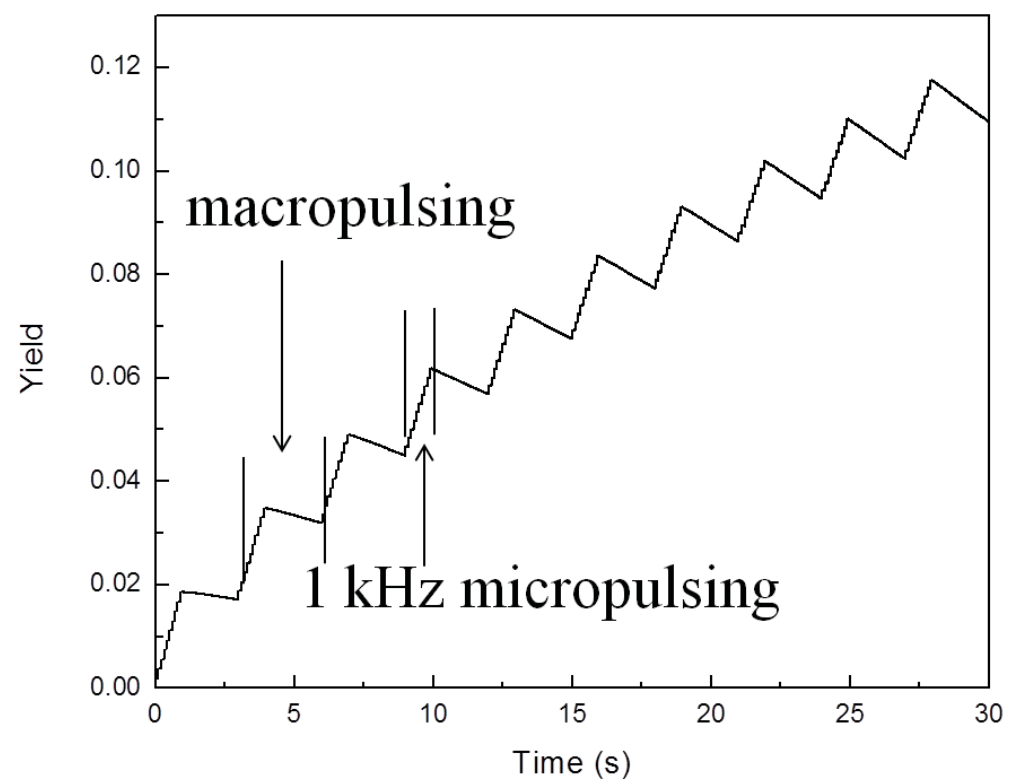

Figure 22. Example of an interlaced pulsing structure. The micropulsing occurs during the On period; the source would be shut off during the second phase of each macropulse.

Previous research, including the initial study conducted for the current project, has shown that the delayed gamma-ray signature is generally stronger than the delayed neutron signature in terms of indicating the presence of fissionable material. As a result, we focused on the gamma-ray emission from fission fragments in order to optimize the pulsing structure. To initiate the optimization process, a simple MCNP6 model was developed wherein a single, instantaneous pulse from the inspection source is assumed, and the delayed gamma rays resulting from ${ }^{235} \mathrm{U}$ induced fission are tallied as a function of time. Rather than relying on the simulation software's gamma-ray emission capabilities, a custom software package was developed to produce the discrete gamma-rays generated from the fission fragments, and to also convolve the timedependent emissions from a single source pulse with an interlaced pulsing structure. This same software package was also relied upon to produce similar time- and energy-dependent delayed neutron distributions; however, only the gamma-ray aspect was utilized while optimizing the pulsing structure. Hence, details specific to the process of generating the gamma-ray distributions are discussed here; the delayed neutron distributions are discussed later.

With the developed software package, an independent fission fragment mass distribution is imported, and a total number of time bins, as well as the total decay time to consider, are selected. The software then generates a corresponding Origen-S file, which is subsequently used to produce a time-dependent distribution of isotopic abundances. From the Origen-S output, the total number of decays for each isotope during each time step is approximated by multiplying the isotopes' activities by the width of the time step. It should be noted that this method assumes that the duration of each time bin is small compared with the half-lives of the respective isotopes, as the chosen Origen-S output has units of curies. With the total number of decays, the software searches the Evaluated Nuclear Structure Data Files (ENSDF) and extracts the discrete gammaray emissions for each isotope at each time step. As an example, consider the isotope ${ }^{84} \mathrm{As}$, which 
is produced with an abundance of 0.0023 atoms per fission (235U, ENDF/B-VII). After two 333.3 ms time steps, Origen-S gives the activity of ${ }^{84} \mathrm{As}$ as $9.19 \times 10^{-15} \mathrm{Ci}$. The software pulls each of the gamma rays associated with ${ }^{84} \mathrm{As}$ decay and adds them, in the correct abundance based on the activity, to an MCNP6-formatted source file. An excerpt from a source file is shown in Figure 23, with the ${ }^{84}$ As gamma rays highlighted.

$\begin{array}{ll}3.0247 & 1.2365807 \mathrm{E}-06 \\ 3.071 & 1.73520195 \mathrm{E}-06 \\ \text { SI } 4 & \text { SP4 } \\ \text { L } & \text { D } \\ 3.6688 & 2.09507566666667 \mathrm{E}-07 \\ 3.03946 & 2.51598298 \mathrm{E}-06 \\ 3.1694 & 6.94890537333333 \mathrm{E}-07 \\ 3.4746 & 8.26680122 \mathrm{E}-07 \\ 3.748 & 6.46967052 \mathrm{E}-07 \\ 4.1279 & 8.14699250666667 \mathrm{E}-07 \\ 4.2809 & 7.1885228 \mathrm{E}-07 \\ 4.9457 & 1.61741763 \mathrm{E}-06 \\ 5.0868 & 8.26680122 \mathrm{E}-07 \\ 5.1499 & 1.06629754866667 \mathrm{E}-06 \\ 3.0454 & 2.6262045 \mathrm{E}-11 \\ 3.2353 & 2.1009636 \mathrm{E}-11 \\ 3.3658 & 3.04639722 \mathrm{E}-11 \\ 3.9275 & 7.14327624 \mathrm{E}-11\end{array}$

Figure 23. MCNP6 source file excerpt showing the ${ }^{84} \mathrm{As}$ gamma rays and their abundances, per fission, $667 \mathrm{~ms}$ after fission.

This process is completed for every fission fragment (and their subsequent daughters) at each time step. Once completed, the source file is included in a final simulation, which tracks the gross gamma-ray flux incident on the modeled detectors at each time step. The result (see Figure 24) is a time-dependent, gross gamma-ray yield distribution, from which all gamma rays with energies below $3 \mathrm{MeV}$ have been excluded.

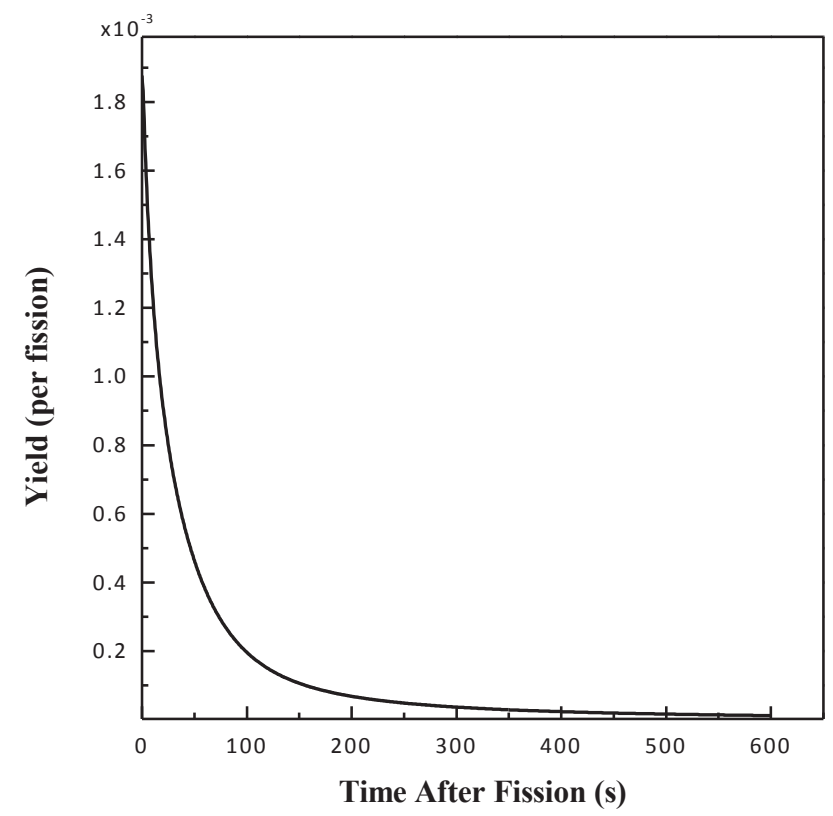

Figure 24. Time-dependent, gross gamma-ray yield ( $E_{\gamma} \geq 3 \mathrm{MeV}$ ) per fission incident on an arbitrary detector surface. 
As a final step, the software package takes the yield distribution and convolves it with an interlaced pulsing structure for various combinations of $\mathrm{On}$ and Off times. A representative result is shown below in Figure 25, which gives the gross gamma-ray yield from fission fragments versus On time (macro pulse width) and Off time (count time between macro pulses). From these calculations, the optimal ratio, regardless of the On time, appears to generally follow a 1:1 On to Off ratio. We also note that these calculations assume a $30.3 \mathrm{~ms}$ prompt region. Depending on the environment for a given scenario, the prompt region may have to be extended much further in time, ultimately resulting in optimal On and Off times that deviate from a 1:1 ratio. Nevertheless, such a deviation would likely be location and environment specific. Thus, the 1:1 ratio shown to be optimal for the prompt regions considered here was used here.

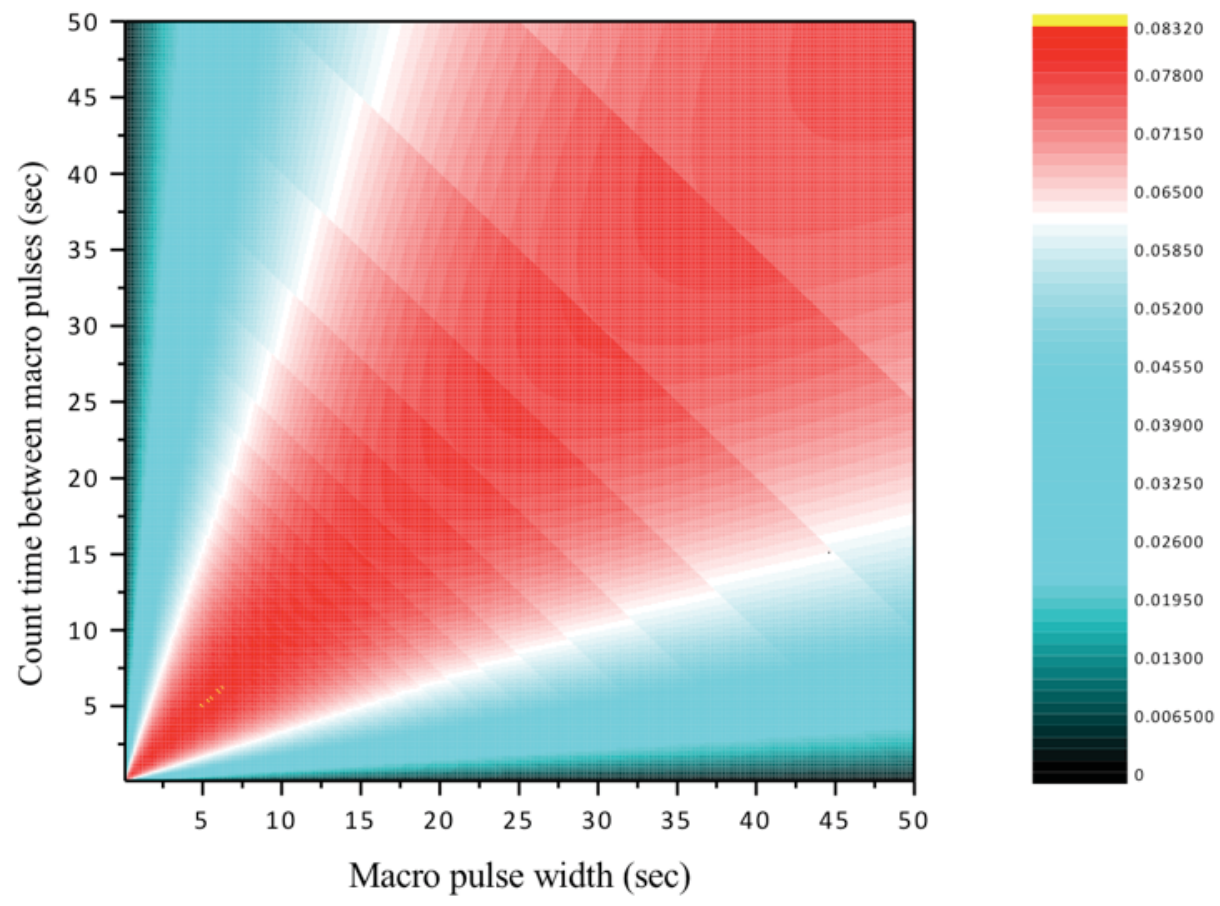

Figure 25. Relative gross delayed gamma-ray yield from fission fragments versus macro pulse width (On time) and count time between pulses (Off time). These calculations assumed a $1 \mathrm{kHz}$ micropulsing rate and a $300 \mathrm{~s}$ total measurement time.

For the delayed neutron yields, convolving the time-dependent gross yields incident on the detector was completed analogously with the delayed gamma rays. The time- and energydependent distributions for delayed neutron emission were not taken from the fragment distributions and ENSDF database. Rather, a six group delayed neutron scheme was assumed, with the corresponding data from ENDF/B-VII. The six group scheme is based on an average of $\sim 270$ known delayed neutron precursors, which are combined into one of six groups based on their half-lives. Each of the six groups then has a corresponding decay constant, group abundance, and associated energy distribution. The energy distribution of each group is shown below in Figure 26. The data shown in the figure are normalized such that the total yield for each individual group is set to unity. 


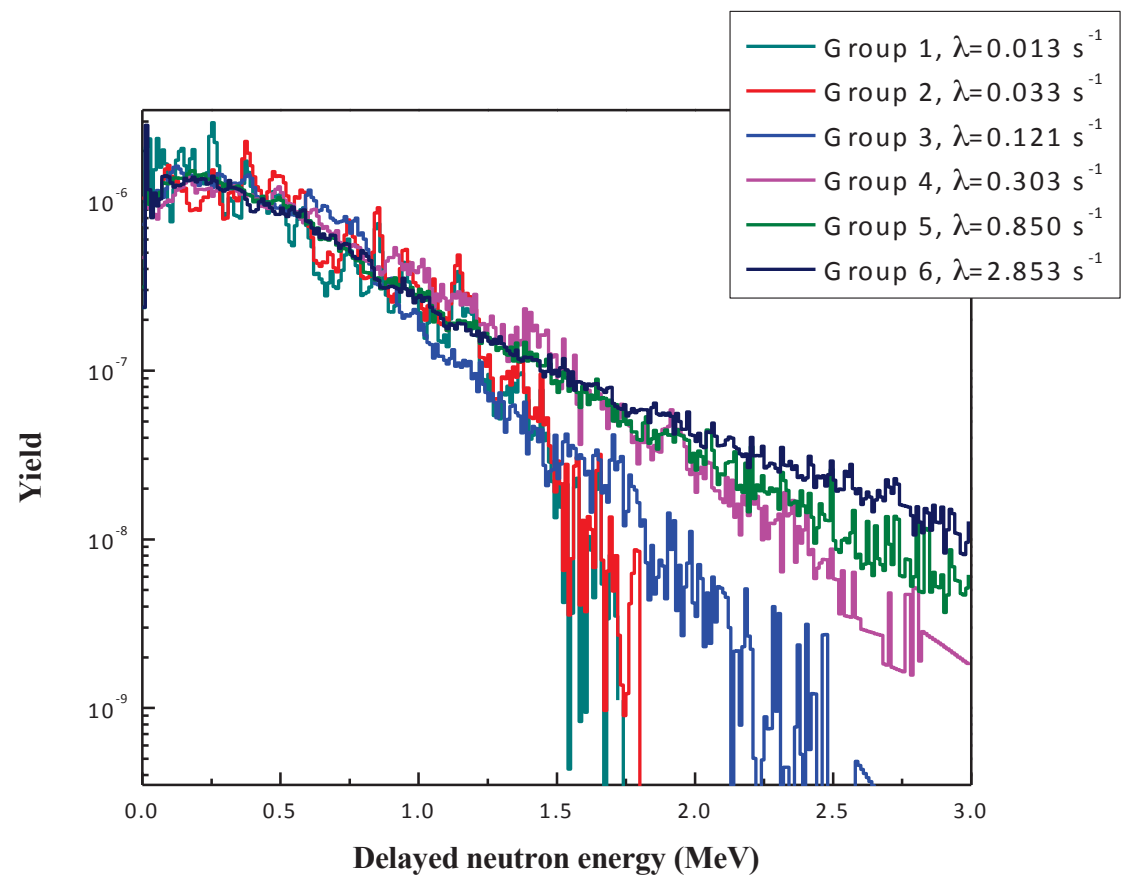

Figure 26. Energy distributions for each of the six delayed neutron groups. The distributions are normalized so that the sum of yields for each group equal 1.

In contrast to the delayed gamma rays and delayed neutrons, an interlaced pulsing structure would not be required for prompt neutrons, assuming maximum source repetition rates of $\sim 1$ $\mathrm{kHz}$ and distances similar to those considered here (tens of meters). Hence, for assessing the prompt neutron signature, it was assumed that the source would be pulsed continuously for the entire inspection period. Prompt neutron emission rates, per fission, and their respective energy distributions relied on the LLNL fission model [89].

The MPS parameter simulations established appropriate beam settings to be used for each signature to maximize potential signal. The result indicates that significantly broader energy spread is acceptable than was used in initial calculations. Correspondingly, this means that appropriate photon dose can be delivered at kHz-class repetition rates instead of the $15 \mathrm{kHz}$ indicated by initial assessments.

\section{Simulation Results for Detection of Hidden SNM}

The simulations assessed each inspection object configuration and set of shielding conditions, using the optimized MPS beam parameters described above. Initial simulations were completed, during which the inspection beam was modeled and transported to calculate the total number of fission reactions that were induced (photofission and secondary, neutron-induced fission), as well as to track the location of fission sites within each inspection object. An example is given in Figure 27, which shows a cross section of an inspection object and the induced fission sites for an unshielded object in Configuration A. As can be seen in the figure, the majority of the fission reactions occur in the first few centimeters of the object, on the side facing the inspection source. In an effort to more precisely mimic any self-shielding effects, the locations of the fission sites were then used as the source locations in subsequent simulations, for which the prompt neutrons, delayed gamma rays, and delayed neutrons were transported to the detectors as a function of time after the inspection beam pulse. These simulations were conducted for each object and shielding configuration to evaluate detection. 


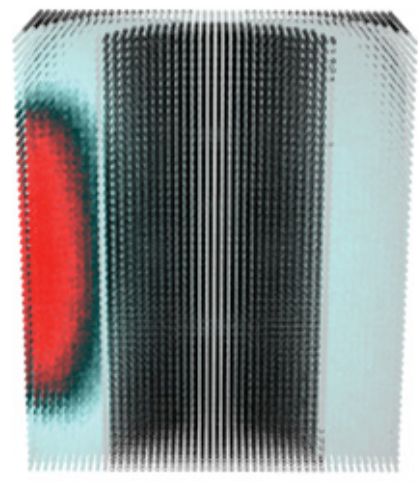

Figure 27. Cross-section of inspection object showing induced-fission sites with Configuration A.

The first set of simulations established the appropriate macropulse repetition rate. Figure 28 shows the simulation results for Configuration A shielded with varying thicknesses of steel; the detected net yield for delayed gamma rays is plotted as a function of macropulse repetition rate. For these simulations, the On and Off times were set to a 1:1 ratio as determined above, micropulse repetition rates were $1 \mathrm{kHz}$, and the total measurement time was assumed to be limited to $300 \mathrm{~s}$. Due to the characteristic decay of fission fragments, the maximum net yields decrease as the macropulse repetition rate increases. As an example, the detected net yield for 1 $\mathrm{Hz}$ macropulsing is well above the critical decision level; however, as the macropulse rate increases the net yield steadily decreases, and it falls below the decision level for repetition rates greater than $\sim 13 \mathrm{~Hz}$.

Although the specific macropulse repetition rate for which an individual case falls below the critical decision levels varies, the general trend of decreasing net yield as a function of increasing macropulse repetition rate was generally consistent. The final results were then calculated by setting the macropulse repetition rate to $1 \mathrm{~Hz}(0.5 \mathrm{~s} 0 \mathrm{n}, 0.5 \mathrm{~s}$ Off). While Figure 28 indicates that the net yield are moderately higher at lower macropulse repetition rates, a $1 \mathrm{~Hz}$ rate allows for short minimum measurement times. Conversely, the signals increase with micropulse repetition rate and with photon yield per shot, which governs total photon flux on target. Simulations presented here are for a $1 \mathrm{kHz}$ rate, and for $8.5 \times 10^{9}$ and $6.9 \times 10^{9}$ photons/pulse for delayed and prompt signatures respectively (as derived above). The results may be scaled to higher (or lower) rates that may be possible. 


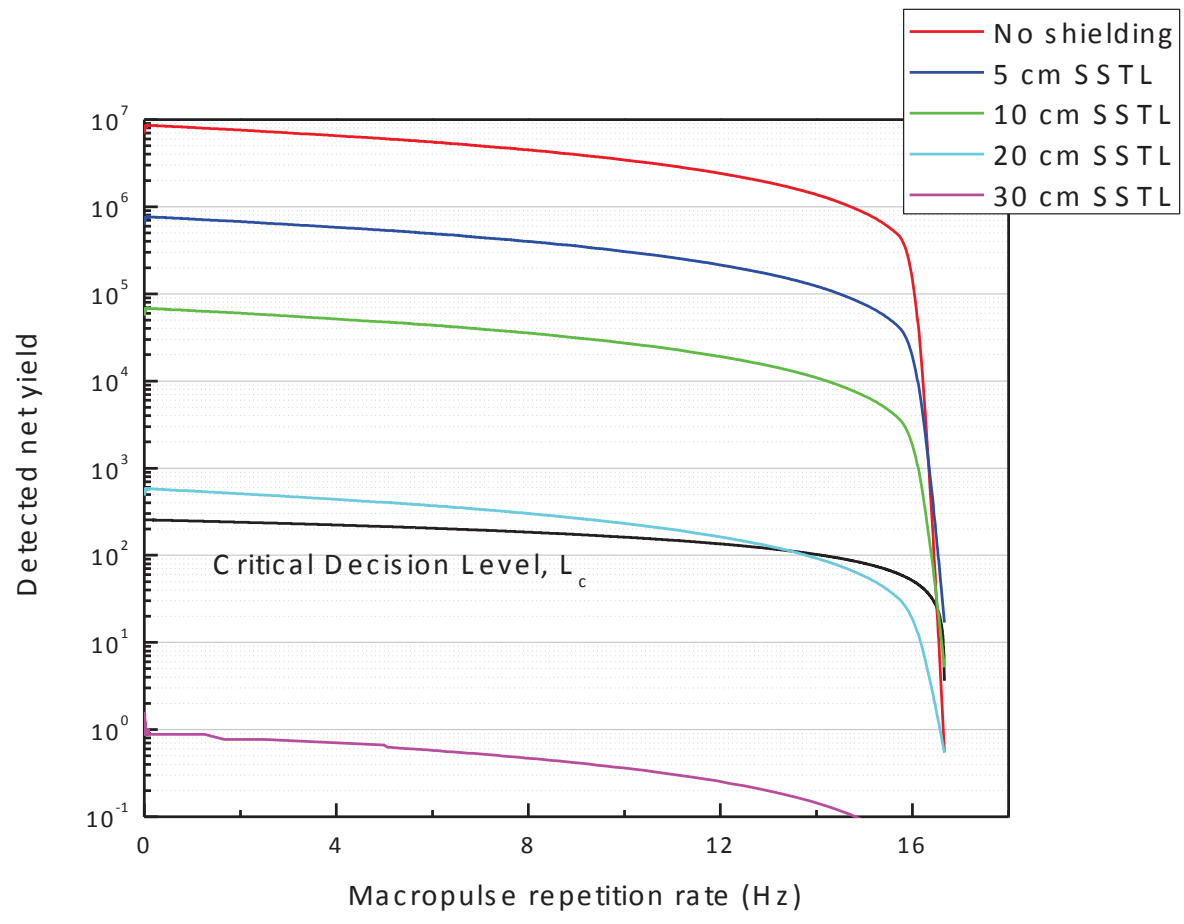

Figure 28. Detected net yield for the delayed gamma-ray signal, with target configuration A and steel shielding, as a function of macropulse repetition rate. On and Off periods were set to a 1:1 ratio. The total time allowed for a measurement was assumed to be 300 seconds.

The detected net yields for the delayed gamma ray, delayed neutron, and prompt neutron signals are given for target configuration $\mathrm{A}$, as a function of shielding thickness for steel (Figure 29), water (Figure 30), and composite (Figure 31).
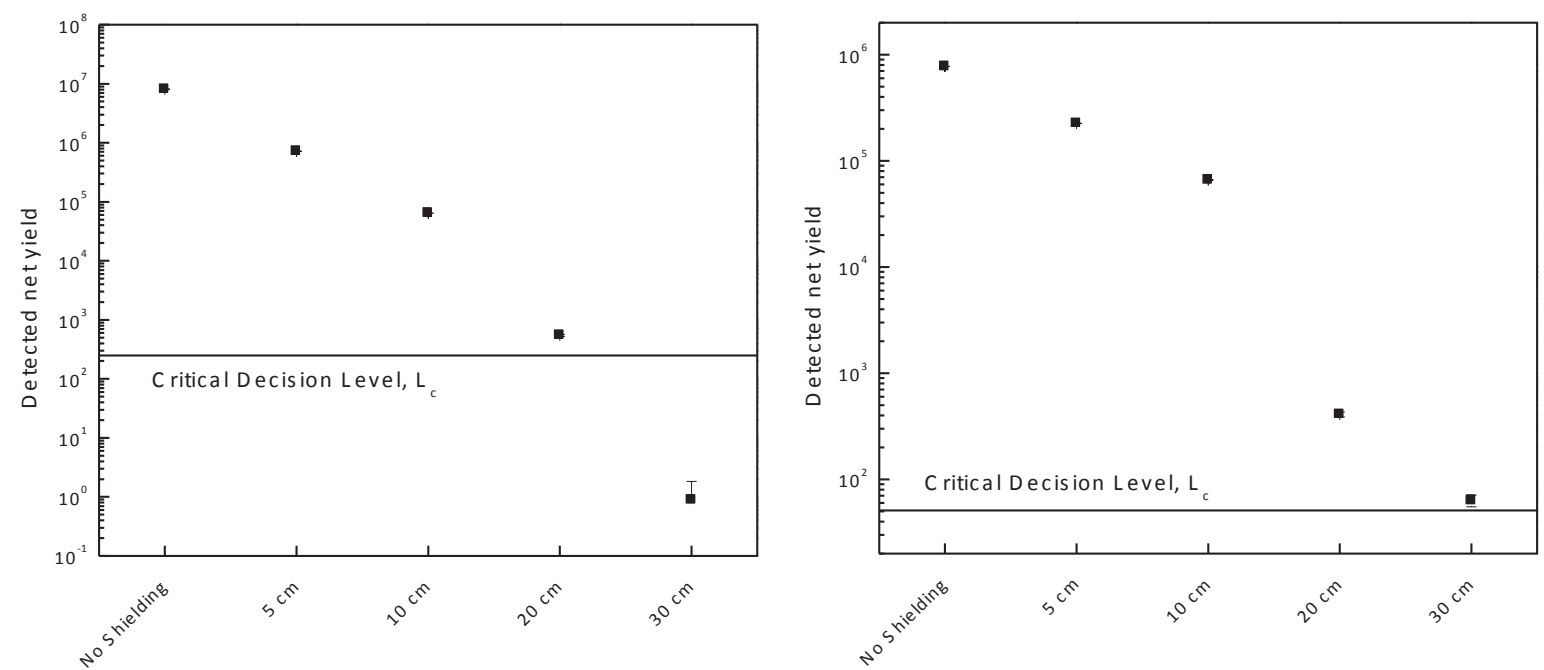

Figure 29a. Detected net yield for the delayed gamma-ray signal (left), and delayed neutron signal (right) with target configuration $A$, as a function of steel shielding thickness. 


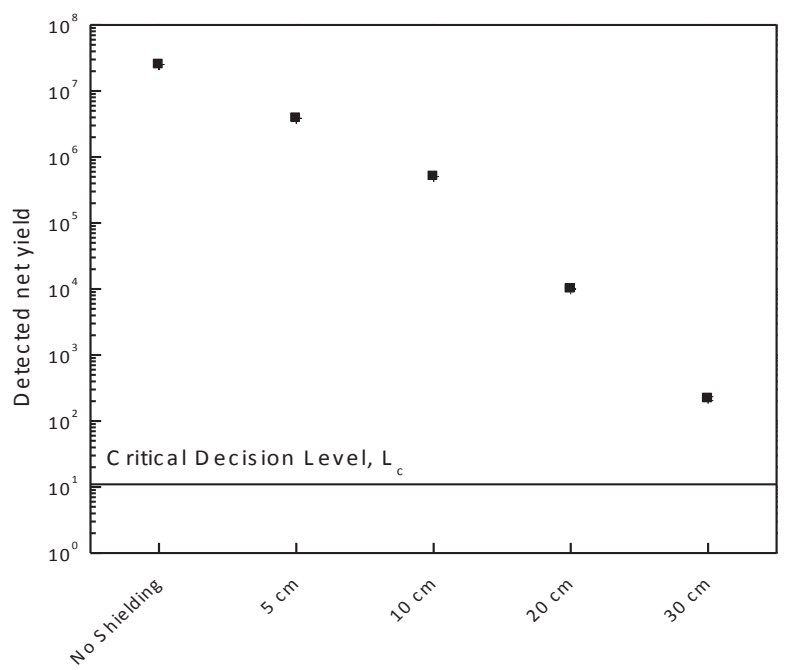

Figure 29b. Detected net yield for the prompt neutron signal with target configuration A, as a function of steel shielding thickness.
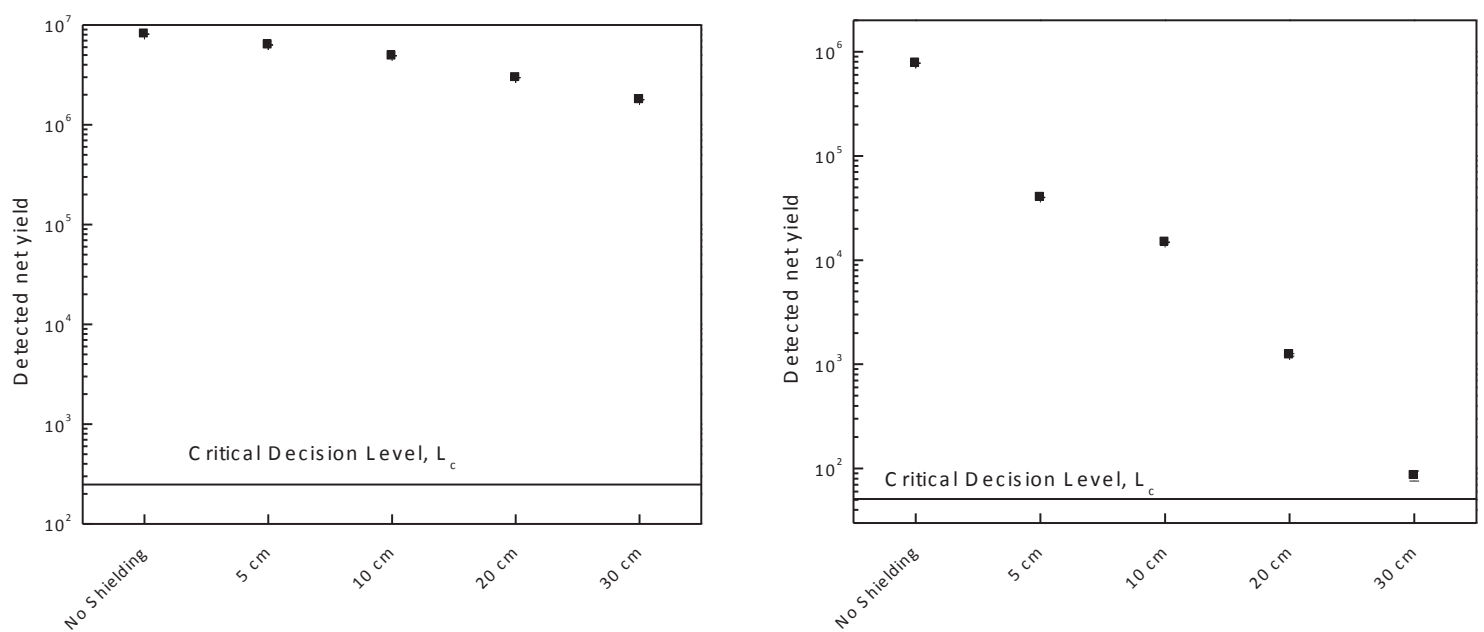

Figure 30a. Detected net yield for the delayed gamma-ray signal (left), and delayed neutron signal (right) with target configuration $A$, as a function of water shielding thickness.

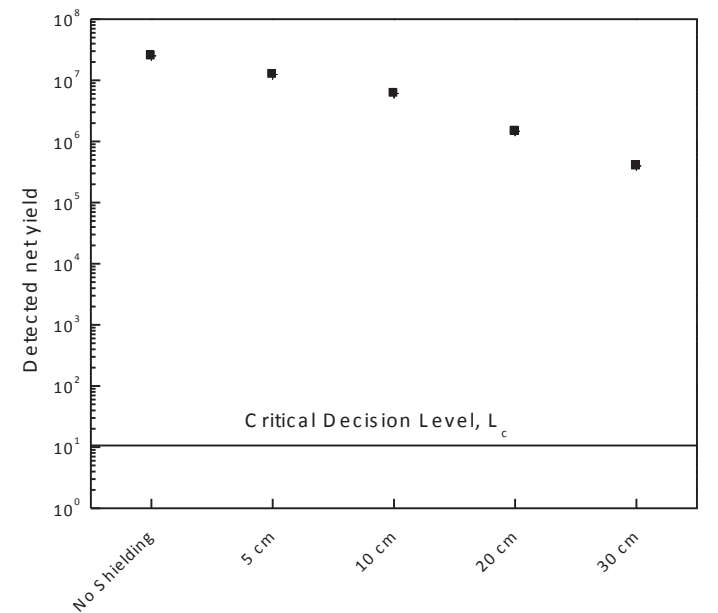

Figure 30b. Detected net yield for the prompt neutron signal with target configuration $A$, as a function of water shielding thickness. 

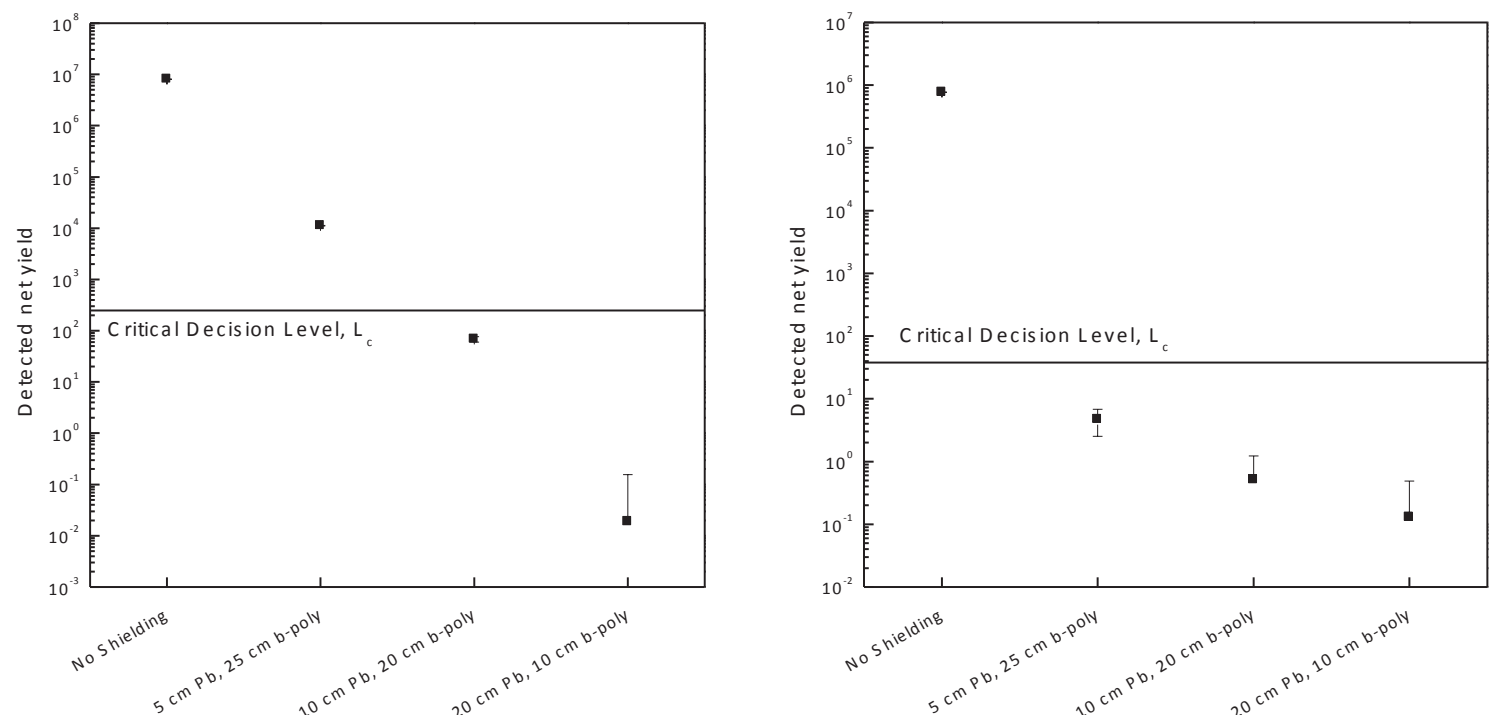

Figure 31a. Detected net yield for the delayed gamma-ray signal (left), and delayed neutron signal (right) with target configuration A, as a function of composite shielding thickness.

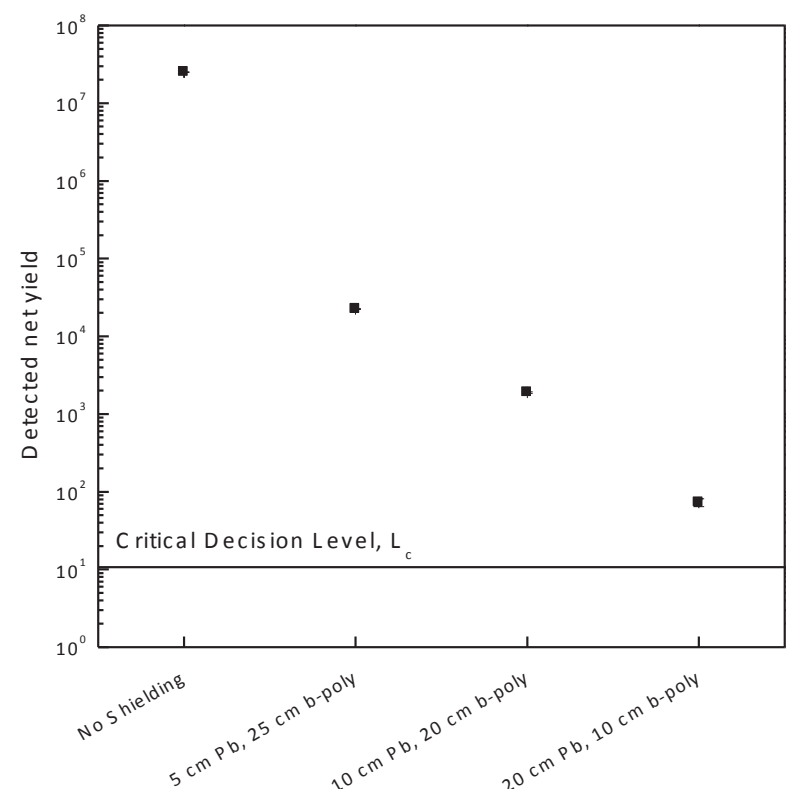

Figure $31 \mathrm{~b}$. Detected net yield for the prompt neutron signal with target configuration $A$, as a function of composite shielding thickness.

For the low-Z material (water) the delayed gamma signal is strong, being $\sim 4$ orders of magnitude above the critical decision level at the maximum simulated $30 \mathrm{~cm}$ thickness. However, for higher- $\mathrm{Z}$ shielding (steel and $\mathrm{Pb}$ in the composite shielding) the delayed gamma signal is strongly attenuated and drops quickly under the threshold. The prompt neutron signal is also $\sim 4$ orders of magnitude above the critical decision threshold for the water shielding but, in contrast to the gamma signal, also stays above the critical decision threshold for all simulated thicknesses of the steel and the composite shielding. This is due to the penetrating capabilities of higher energy neutrons and, importantly, also due to the low critical decision threshold. The neutron background determining the threshold for the prompt signal is low because only neutron events that happen during the prompt counting periods contribute. The prompt neutrons are collected 
within $\sim 1$ microsecond of each beam pulse so that at a repetition rate of $1 \mathrm{kHz}$, only the number of background neutrons arriving in the detector in $1 / 1000$ of the 300 second total measurement time contribute. For this reason the sensitivity of the prompt neutron signal is dramatically higher than in the initial estimate. The delayed neutron signal is in general weaker than the prompt neutron signal, but still produces usable signatures in the water and steel cases simulated, and in the thin composite cases.

Tables 19, 20, and 21 present the results of the simulations for Configurations A, B, and C, respectively. With Configuration $A$, each of the three signatures would correctly indicate the presence of fissionable material through at least $30 \mathrm{~cm}$ of water shielding. Although the delayed gamma ray signature would produce false negatives for steel shielding thickness near $30 \mathrm{~cm}$, the net yields for delayed and prompt neutrons remain above the critical decision level for at least 30 $\mathrm{cm}$ of steel shielding. For the composite shielding cases, only the prompt neutron signature remained above the decision level for all three lead + borated polyethylene cases, also up to 30 $\mathrm{cm}$ thick. Because the primary goal of this study was to indicate what thickness of an unknown material could be penetrated, thicker water or other shielding was not simulated even in cases where the signal levels at $30 \mathrm{~cm}$ were substantially above the decision level.

Table 19: Configuration A, $1 \mathrm{~Hz}$ macropulse repetition rate, $300 \mathrm{~s}$ total measurement time.

\begin{tabular}{|c|c|c|c|c|}
\hline \multirow{3}{*}{ Shield Material } & Thickness $\mathbf{( c m )}$ & $\begin{array}{c}\text { Delayed } \boldsymbol{\gamma} \text {-rays }> \\
\mathbf{L}_{\mathbf{c}}\end{array}$ & $\begin{array}{c}\text { Delayed Neutrons } \\
>\mathbf{L}_{\mathbf{c}}\end{array}$ & $\begin{array}{c}\text { Prompt Neutrons } \\
>\mathbf{L}_{\mathbf{c}}\end{array}$ \\
\hline \multirow{3}{*}{ No Shielding } & N/A & Yes & Yes & Yes \\
\hline \multirow{4}{*}{ Water } & 5 & Yes & Yes & Yes \\
\cline { 2 - 5 } & 10 & Yes & Yes & Yes \\
\cline { 2 - 5 } & 20 & Yes & Yes & Yes \\
\cline { 2 - 5 } & 30 & Yes & Yes & Yes \\
\cline { 2 - 5 } & 5 & Yes & Yes & Yes \\
\cline { 2 - 5 } & 10 & Yes & Yes & Yes \\
\cline { 2 - 5 } & 20 & No & Yes & Yes \\
\hline \multirow{3}{*}{$\begin{array}{c}\text { Composite } \\
\text { (Lead/Borated } \\
\text { Poly) }\end{array}$} & 30 & Yes & Yes & Yes \\
\cline { 2 - 5 } & $10 / 20$ & No & No & Yes \\
\cline { 2 - 5 }
\end{tabular}


For Configuration B, each of the three signatures would correctly indicate the presence of fissionable material through at least $20 \mathrm{~cm}$ of water shielding, with the lone false negative for water shielding occurring with the delayed neutron signature and $30 \mathrm{~cm}$ of water shielding. Each of the delayed signatures produced a false negative for $30 \mathrm{~cm}$ of steel shielding, although the delayed and prompt neutrons were both successful for at least $20 \mathrm{~cm}$ of steel shielding. Due to the relatively small target area subtended by the beam with Configuration $B$, as well as the substantial beam attenuation through the lead shielding, only the delayed gamma-ray and prompt neutron signatures resulted in net yields above the critical decision level for composite shielding with $5 \mathrm{~cm}$ of lead and $25 \mathrm{~cm}$ borated polyethylene. The prompt neutron signature was the only successful one for composite shielding with $10 \mathrm{~cm}$ of lead and $20 \mathrm{~cm}$ borated polyethylene. All other cases resulted in false negatives.

Table 20: Configuration $\mathrm{B}, 1 \mathrm{~Hz}$ macropulse repetition rate, $300 \mathrm{~s}$ total measurement time.

\begin{tabular}{|c|c|c|c|c|}
\hline Shield Material & Thickness (cm) & $\begin{array}{c}\text { Delayed } \gamma \text {-rays }> \\
L_{c}\end{array}$ & $\begin{array}{c}\text { Delayed Neutrons } \\
\qquad>L_{c}\end{array}$ & $\begin{array}{c}\text { Prompt Neutrons } \\
>L_{c}\end{array}$ \\
\hline No Shielding & $\mathrm{N} / \mathrm{A}$ & Yes & Yes & Yes \\
\hline \multirow{4}{*}{ Water } & 5 & Yes & Yes & Yes \\
\hline & 10 & Yes & Yes & Yes \\
\hline & 20 & Yes & Yes & Yes \\
\hline & 30 & Yes & $\mathrm{No}$ & Yes \\
\hline \multirow{4}{*}{ Steel } & 5 & Yes & Yes & Yes \\
\hline & 10 & Yes & Yes & Yes \\
\hline & 20 & No & Yes & Yes \\
\hline & 30 & No & No & No \\
\hline \multirow{3}{*}{$\begin{array}{c}\text { Composite } \\
\text { (Lead/Borated } \\
\text { Poly) }\end{array}$} & $5 / 25$ & Yes & No & Yes \\
\hline & $10 / 20$ & No & No & Yes \\
\hline & $20 / 10$ & No & No & No \\
\hline
\end{tabular}

With Configuration $\mathrm{C}$, all three signatures had net yields greater than the respective decision levels for all water shielding thickness and at least $20 \mathrm{~cm}$ of steel shielding. Only the prompt neutron signature was successful for the $30 \mathrm{~cm}$ of steel shielding. Similarly, both delayed signatures were unsuccessful when at least $20 \mathrm{~cm}$ of lead shielding was present. The delayed neutron signature produced false negatives for all thicknesses of composite shielding, while the delayed gamma-ray signature was successful for only the $5 \mathrm{~cm}$ lead $+25 \mathrm{~cm}$ borated polyethylene case. The prompt neutron signature was the only one successful for all composite shieldings. 
Table 21: Configuration C, $1 \mathrm{~Hz}$ macropulse repetition rate, $300 \mathrm{~s}$ total measurement time.

\begin{tabular}{|c|c|c|c|c|}
\hline \multirow{2}{*}{ Shield Material } & Thickness $\mathbf{( c m )}$ & $\begin{array}{c}\text { Delayed } \boldsymbol{\gamma} \text {-rays }> \\
\mathbf{L}_{\mathbf{c}}\end{array}$ & $\begin{array}{c}\text { Delayed Neutrons } \\
>\mathbf{L}_{\mathbf{c}}\end{array}$ & $\begin{array}{c}\text { Prompt Neutrons } \\
>\mathbf{L}_{\mathbf{c}}\end{array}$ \\
\hline \multirow{3}{*}{ No Shielding } & N/A & Yes & Yes & Yes \\
\hline \multirow{4}{*}{ Water } & 5 & Yes & Yes & Yes \\
\cline { 2 - 5 } & 10 & Yes & Yes & Yes \\
\cline { 2 - 5 } & 20 & Yes & Yes & Yes \\
\hline \multirow{4}{*}{ Steel } & 30 & Yes & Yes & Yes \\
\cline { 2 - 5 } & 5 & Yes & Yes & Yes \\
\cline { 2 - 5 } & 10 & Yes & Yes & Yes \\
\hline \multirow{2}{*}{$\begin{array}{c}\text { Composite } \\
\text { (Lead/Borated } \\
\text { Poly) }\end{array}$} & 20 & No & No & Yes \\
\cline { 2 - 5 } & $10 / 20$ & Yes & No & Yes \\
\cline { 2 - 5 } & $20 / 10$ & No & No & Yes \\
\hline
\end{tabular}

\section{II.2.5. Impact of and Requirements for MPS Systems}

Positively identifying the presence of fissionable material was shown to be realistic at distances of $\sim 20 \mathrm{~m}$, through shielding thickness up to $30 \mathrm{~cm}$, using an MPS delivering in the range of $10^{12}$ photons/second into a narrow divergence beam (few-mrad opening angle). This could represent an important capability. Operating an MPS at $13.1 \pm 25 \% \mathrm{MeV}\left(8.5 \times 10^{8}\right.$ photons per source pulse) for delayed signatures and $6.7 \pm 20.5 \% \mathrm{MeV}\left(6.9 \times 10^{8}\right.$ photons per source pulse $)$ for prompt neutrons maximizes fission production, taking into account realistic trade-offs of energy spread and yield, without generating problematic levels of source-induced background or signature interferences. For the shielding materials considered, prompt neutrons provide the most sensitive fission signature using an MPS. A readily mobile MPS platform would likely be required for this application. The definition of mobility will vary by application: for some a truckmounted system may be acceptable while others will require a smaller source.

A wider parameter range can be evaluated by appropriate scaling from the specific set of beam parameters, geometry and shielding configurations simulated. The detected gamma and neutron rates scale linearly with the source intensity, i.e., with the repetition rate and the number of photons per pulse. For example operating the source at $10 \mathrm{kHz}$ instead of the assumed $1 \mathrm{kHz}$ would increase gamma and neutron signal rates by a factor of 10 . This may allow penetration of an additional $\sim 10 \mathrm{~cm}$ of steel, or equivalent attenuation depth of other materials. Furthermore, the detected yields scale with the detector size and inversely with the square of the detector to SNM distance, and this could offer another factor of 10 in signal for distances at the $6 \mathrm{~m}$ scale (vs. the assessed $20 \mathrm{~m}$ ). Again, this may allow penetration of an additional $\sim 10 \mathrm{~cm}$ of steel, or equivalent attenuation depth of other materials. To first order the source to object distance does not impact the signal rate as long as the photon beam is sufficiently narrow that the full beam hits the nuclear material. As the simulation results indicate, narrow beam divergence and high photon flux provide limited standoff capability with the decrease in signal rate with detector to target distance being the limiting factor. The object of interest also plays a role, but this effect is not anticipated to change the fundamental conclusions.

The results inform the size of a shielded object within which absence could be determined, and indicate that such a measurement could be realistic in objects at the $\sim 1 \mathrm{~m}^{3}$ size range using 
the source identified. A narrow-angle MPS consistent with parameters anticipated from Thomson source development (i.e., few-mrad divergence) is evaluated as important for enabling this measurement.

Even with a narrow-divergence source, localization and coarse size determination of a shielded object of interest is crucial to allow determination of absence. An appropriate backscatter measurement may expedite measurements by identifying high-Z or high-density areas, consequently localizing a region of interest. Such localization would potentially allow the inspected volume to be increased for the same maximum inspection time, and further allow an inspection system to verify the absence of material rather than simply to detect the presence of material. An in-depth analysis regarding integrating Compton backscatter imaging or similar techniques was outside the scope of the current project, but backscatter work has been conducted previously and appears to be a workable solution. Further work to understand its potential with pulsed, narrow angle MPSs would appear warranted. In some application areas, other techniques such as building radar, or even visual identification, may also be of use for localization though these would not provide the $\mathrm{Z}$ information available from backscatter.

The requirements for use of an MPS-based system for an SNM search application may not be as stringently defined as for other applications. For instance, the acceptable measurement time is likely to be scenario-dependent based on the environment, location, and/or operational considerations. If dose at the target location, or on the environment surrounding the target, is not of primary concern, then producing the highest rate of source photons within a limited energy range would minimize the time required to detect a material of interest. It is to be expected, however, that dose downstream of the source location is likely to be an important consideration. An MPS delivers significant advantage in dose, with direct dose reductions in the range of 2.4-4x due to more efficient excitation of fission. The narrow, mrad divergence of a Thomson source could offer additional dose reduction (configuration dependent) by targeting dose to the object of interest, and would reduce scatter dose to surroundings. 


\section{II.3. Treaty/Dismantlement Verification}

\section{II.3.1. Introduction}

Existing treaties verify delivery systems or count warheads on a delivery system, but do not involve measurements on individual nuclear warheads. As nuclear stockpiles reduce, it is anticipated that the need to verify nuclear warheads will increase to maintain confidence in the dismantlement process. The Office of Nuclear Verification is funding research and development efforts to prepare for this eventuality

Verification of nuclear warheads and their dismantlement is a high priority for treaty verification that presents unique challenges to any measurement approach. The technique should not damage the warhead nor reveal any sensitive information, must be safe for personnel, be verified to function exactly as designed, and perform the intended measurements. Conventional wisdom assumes that the equipment is controlled by the host, who will likely have unmonitored access to the equipment. This latter constraint places a significant burden on the ability to authenticate the equipment. These requirements drive the development toward simple technologies, which may present a challenge for deployment of a MPS in a treaty regime.

Verification of monitored dismantlement can be divided into three distinct objectives:

1. Warhead confirmation: confirming that an item presented for inspection is a warhead.

2. Dismantlement confirmation: confirming that the warhead has been dismantled. The conventional definition of dismantlement is the separation of fissile material (FM) from high explosives (HE).

3. Detection of material diversion: the detection of small amounts of HE or FM in containers that are not supposed to hold those materials.

Compared to other applications (e.g., cargo, detection), Treaty Verification scenarios typically involve less shielding of the object of interest and tolerate longer measurement times. Both detection of materials, which requires less statistical precision, and characterization of materials, which requires more statistical precision, are of interest. There are a variety of constraints on a measurement system for treaty verification. The constraints are due largely to safety and information security concerns. For a more thorough discussion, see references [90,91].

Initial assessment based on scaling of previous measurements and simple slab geometries indicated that the potential impact of MPS-enabled techniques for treaty verification measurements could be strong. Potential scenarios include enabling warhead confirmation as well as enabling a unique identifier for warheads and components. Initial assessment indicated potential benefit for both photofission and NRF methods, and derived general MPS parameter ranges. These ranges were used as input to detailed modeling.

Higher-fidelity calculations and modeling were conducted to evaluate each technique for treaty verification. This section begins with a high-level summary of each of the techniques and potential signatures. The signature assessment simulations indicate that NRF is the most suitable signature. The subsequent subsections present detailed simulations quantifying benefit for several potential usage scenarios. Required intensities are derived, and scalings of performance versus source energy spread are discussed. The nominal MPS simulated is assumed to be operating at $10 \mathrm{kHz}$ with a 2\% FWHM energy distribution unless otherwise noted, based on initial calculations. Radiography techniques relevant to treaty verification may be developed and verified first in support of stockpile stewardship, which was therefore included in the assessment as a separate section, II.4. 


\section{II.3.2 Treaty/Dismantlement Verification Problem Definition and Signatures}

Our approach to assessing the potential impact of MPSs on Treaty/Dismantlement objectives is based on the time-to-detection for specific scenarios. The details of the scenario depend on the objective. Both photofission and NRF signatures were evaluated for representative objects.

To evaluate material characteristics, which may be of interest for warhead confirmation, the scenarios utilized observation of specific materials for different Idaho National Laboratory (INL) Inspection Objects (IOs). [89] The INL IOs offer a range of material characteristics to test the capability of our experimental setup. If we can successfully determine the challenging characteristics of the INL inspection objects we have a greater confidence that the system will also work for actual or purported weapons and/or weapon components. For dismantlement confirmation, the challenge is the detection of small amounts of FM with larger amounts of HE and smaller amounts of HE with larger amounts of FM. For diversion detection, we additionally consider the possibility of additional shielding that would make the observation of the clandestine material more challenging. In all cases, we assumed a probability of detection of $99 \%$ and a probability of false alarms of $1 \%$ in determining the time-to-detection.

We initially considered three different physics process that could be observed using an MPS for treaty verification: radiography/imaging, photofission and nuclear resonance fluorescence (NRF). An MPS may provide multiple advantages for conducting radiography or tomography measurements. Monoenergetic photons, the resulting reduction in scattering, and beams with narrow angle and/or small emission spot size may be combined to significantly improve on bremsstrahlung-based approaches currently deployed. MPS-based radiography for stockpile stewardship has the potential provide micron-scale spatial resolution. This capability may have eventual utility for weapons fingerprinting in the treaty/dismantlement context. This approach may require management of classified information but would provide very specific information. One potential development pathway is to initially develop an MPS-based imaging system in support of stockpile stewardship, and then once the nuclear enterprise has experience with the approach it can consider adaptations in support of treaty verification applications. For this reason, a detailed discussion of radiography is presented in the stockpile stewardship section. For the present analysis of treaty verification, we assume that only crude transmission radiography measurements are performed to isolate regions of interest for more detailed interrogation using photofission or NRF. The concept behind photofission was to use the different energy dependence of the photofission cross sections for ${ }^{235} \mathrm{U},{ }^{238} \mathrm{U}$ and ${ }^{239} \mathrm{Pu}$ to differentiate these materials. We show below that this approach is not viable for treaty verification. The final physics process considered was NRF, which provides an isotope-specific signature for many isotopes. Beyond the discussion below on photofission, the remainder of this section is focused on evaluating the potential of MPS-based NRF measurements, as well as a comparison to current capabilities using bremsstrahlung-based photon sources.

Photofission measurements are sensitive to fissile material in the test object, but do not provide a direct signal for identification of high explosives (HE). The original concept for utilizing photofission in treaty verification was to use the differences in photofission cross section between ${ }^{235 \mathrm{U}},{ }^{238} \mathrm{U}$ and ${ }^{239} \mathrm{Pu}$ at multiple energies to differentiate between these materials. Each of these materials has a significant photofission cross section, so that the observation of fission neutrons alone is not adequate to separate the FM from ${ }^{238 U}$. The energy dependence of the observable neutron yields from these materials is shown in Figure 32, which suggests that measurements over different energy regions might be combined in a manner to provide sensitivity to the three different isotopes. Such measurements would be very challenging, if not impossible, with a bremsstrahlung source because of the averaging over a wide range of photon energies, effectively smearing out the isotopic differences. 

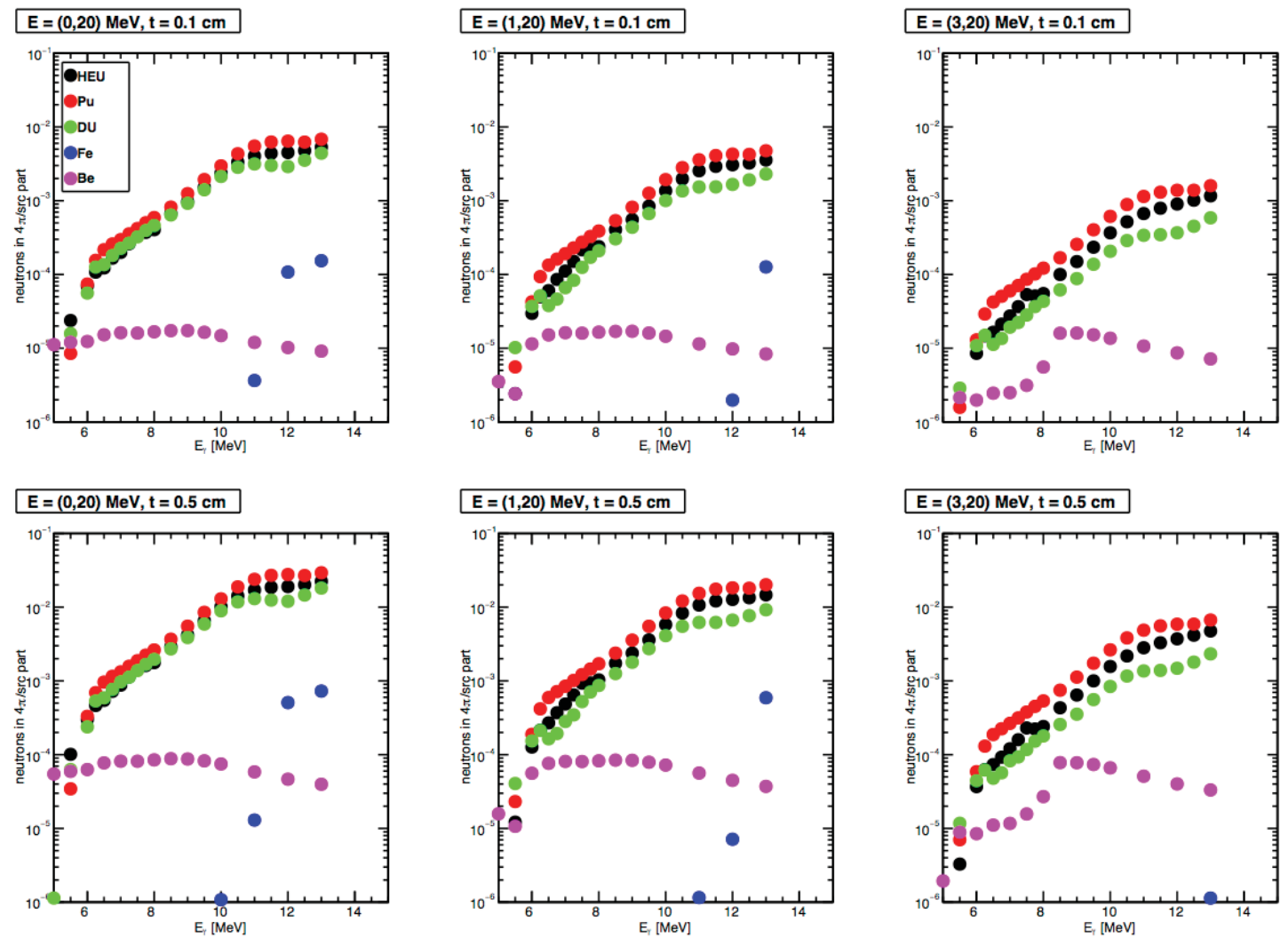

Figure 32. Neutron yields for various target materials as a function of interrogating photon beam energy from an MCNP simulation. The top row is for materials $1 \mathrm{~mm}$ thick, while the bottom row is for materials $5 \mathrm{~mm}$ thick. The left column is for all energy neutrons. The center column is for neutrons with energy greater than 1 $\mathrm{MeV}$, which will be sensitive to fission neutrons and photo-neutrons for some materials. The left column is for neutrons with energy greater than $3 \mathrm{MeV}$, which is sensitive mostly to fission neutrons.

The challenge with this approach for material verification is that the order of materials is unknown. Suppose that the treaty accountable item (TAI) contains both depleted uranium (DU) and weapons grade plutonium (WGPu), but in unknown amounts and in unknown order with respect to the beam. Since the order of the materials is unknown, the approach could be viable if the neutron yield for both orientations, Pu first-DU second and DU first-Pu second, is the same. Table 22 shows the neutron yield over all solid angles for various configurations, which differ for different material orders. For instance, the neutron yield for $1 \mathrm{~mm}$ of Pu upstream of $1 \mathrm{~mm}$ of DU downstream is roughly 4 times the neutron yield for the materials in the opposite order. The

Table 22: Neutron yields from $6.5 \mathrm{MeV}$ photons incident on different target configurations from MCNP simulations.

\begin{tabular}{|c|c|c|}
\hline & \multicolumn{2}{|c|}{$\begin{array}{c}\text { Neutron Yield } \\
\text { (neutrons/src particle) }\end{array}$} \\
\hline & $1 \mathrm{~mm}$ & $5 \mathrm{~mm}$ \\
\hline Pu First, Du Second & $4.3 \cdot 10^{-5}$ & $1.9 \cdot 10^{-4}$ \\
\hline DU Second, Pu First & $1.1 \cdot 10^{-5}$ & $4.7 \cdot 10^{-5}$ \\
\hline
\end{tabular}

differences observed in neutron yields for different material ordering are due to the attenuation of the photon beam by the first material when it reaches the second material. Furthermore, the neutron yield of $1 \mathrm{~mm}$ Pu upstream of 1 $\mathrm{mm}$ of DU downstream is very similar to $5 \mathrm{~mm}$ DU upstream and $5 \mathrm{~mm} \mathrm{Pu}$ downstream. One possible means for photofission to still be viable would be 
if the angular distribution differed for the ordering of the materials; however, the angular distribution was found to be the same within 1-3\% statistical uncertainties of the simulations. The final possible approach for photofission to be viable for treaty verification would be to conduct more than three measurements as a means to provide more information to help separate the thickness of types of material (i.e., to trace out the curves shown in Figures 33 and 34 with high energy resolution). The propagation of uncertainty for three or more different energy measurements presented a challenging scenario to resolve material thicknesses, so that adding more measurements is likely to only increase that challenge. For these reasons, photofission was viewed as not a feasible approach at this time for treaty verification because of the difficulty of separating DU from FM. Photofission may still be a viable approach for detection and or characterization of a single material, or if the order of the materials is known.

Further simulations were performed with MCNPX to examine isotopic discrimination of $235 \mathrm{U}$, ${ }^{238} \mathrm{U}$ and ${ }^{239} \mathrm{Pu}$ single materials using multi-energy MPS probing of photofission cross sections over a wider energy range up to $19 \mathrm{MeV}$. Total fission rates were calculated for $1 \mathrm{~kg}$ spheres of either HEU, DU or ${ }^{239} \mathrm{Pu}$ at 14 different monoenergetic beam energies of photons. [92] The fission rate values are plotted as function of the photon beam energy in Figure 33. The shape of the curve between 10.5 and $13.5 \mathrm{MeV}$, and the magnitude of the curve above $13.5 \mathrm{MeV}$, show discrepancies between fissile and non-fissile materials that, in principle, could be used to distinguish between the isotopes of interest.

Several shielded cases were tested along with the bare case to include problems of beam suppression and neutron reflection. Even in the most challenging case, shown in Figure 34, features in the fission rate curve were observed that were similar to those seen in the unshielded case. However, experimental observables are fission neutron and photon rates and not the fission rates shown in Figures 33 and 34. In the energy range considered here, the $(\gamma, n)$ cross sections are comparable to the fission cross sections thus impacting the observed signature. Furthermore, at energies around $12 \mathrm{MeV}$ and above, the $(\gamma, 2 \mathrm{n})$ channel is open, further diluting the observable differences in fission cross sections. Therefore, while these calculations illustrate differences in the energy dependence of the photofission rates of these three materials that could be exploited for single materials, application-specific simulations that include all contributions to observable signatures would be needed to assess this method if a desired use case were identified.

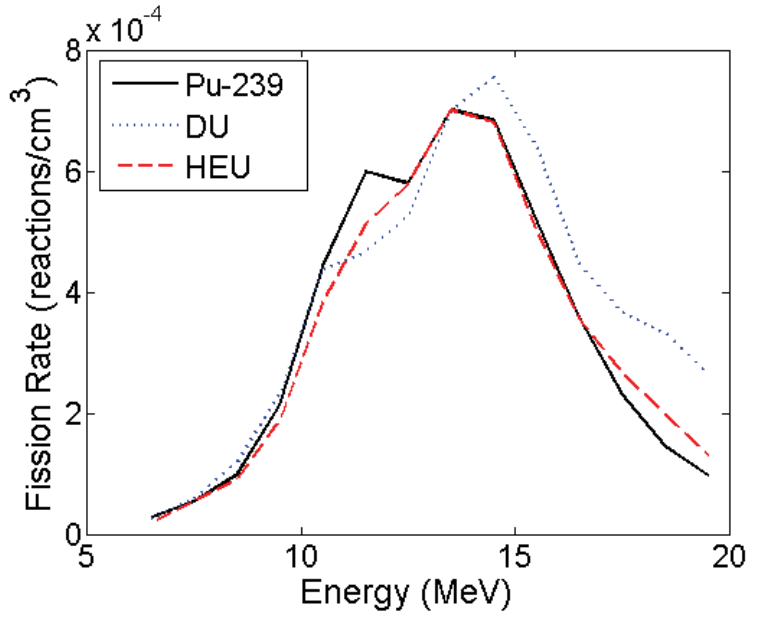

Figure 33. Scaled fission rates in bare 1 $\mathrm{kg}$ spheres of material.

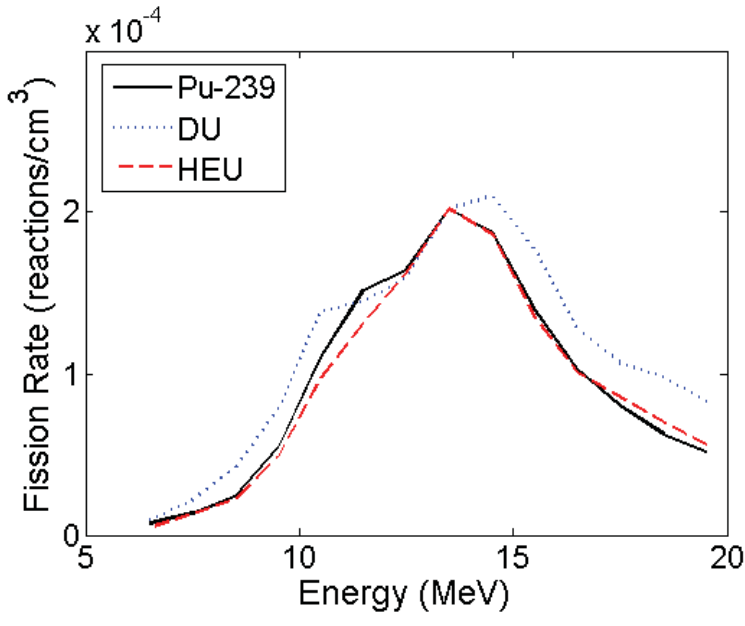

Figure 34. Scaled fission rates in $3 \mathrm{~cm}$ lead and $5 \mathrm{~cm}$ polyethylene shielded $1 \mathrm{~kg}$ spheres of material. 
NRF measurements provide isotope-specific information, and could play a significant role in warhead confirmation and dismantlement confirmation. It is possible to identify and characterize individual isotopes, enabling NRF techniques to be used for measurements related to both fissile materials and HE. The principal advantages of an MPS source compared to a bremsstrahlung source are lower dose and faster measurements. NRF transmission measurements using MPSs can further be designed to limit the sensitivity to specific isotopes, providing a physical information barrier. For instance, a measurement can be designed to be sensitive to ${ }^{239} \mathrm{Pu}$ but not ${ }^{240} \mathrm{Pu}$ (important because the ${ }^{240} \mathrm{Pu} /{ }^{239} \mathrm{Pu}$ ratio is sensitive to a likely treaty partner), thereby reducing the need for an electronic information barrier to protect sensitive information. $[17,21]$ In addition, the narrow energy response of MPSs restricts the potential observables that are available, providing additional information protection. NRF techniques, either scattering or transmission, could be used to measure the amount of carbon, nitrogen and oxygen in the sample as a means to detect HE. A MPS would be particularly beneficial compared to a bremsstrahlung source for measuring the $\mathrm{NRF}$ lines of oxygen, which are around $7 \mathrm{MeV}$. Bremsstrahlung-based NRF measurements are generally detector-rate limited, which becomes increasingly problematic as the end-point energy increases and thus the fraction of events contributing to the signal in the detector decreases.

$\mathrm{NRF}$ techniques present two measurement challenges. First, the rates from NRF processes are generally low, requiring either many detectors or a high beam flux to provide an adequate counting rate for making measurements in a reasonable period of time. For comparison, a bremsstrahlung photon source designed for detecting the $1733-\mathrm{keV}$ line of $235 \mathrm{U}$ using a $2.1 \mathrm{MeV}$ end-point energy can provide on the order of 20 photons $/ \mathrm{eV} / \mu \mathrm{C} / \mathrm{mSr}$, where the typical beam current can range from 1 to $100 \mu \mathrm{A}$. The second challenge is that NRF measurements can be limited by the maximum instantaneous detector rate; this concern is particularly true for bremsstrahlung-based measurements, which typically address the counting-rate challenge by using a continuous-wave beam ${ }^{2}$. The detector rate issue will be less problematic for a MPS, since there is less low energy background dominating the detector rate.

The primary differences between the evaluations of the different treaty objectives are the geometries modeled. To examine the effect on measurement response to the presence of different materials, INL IO\#5, IO\#8 and IO\#10 were evaluated, as they contain both high density and low density materials in the form of HEU, WGPu, and polyethylene. To examine potential capabilities in the context of dismantlement confirmation, separate geometries were evaluated for confirmation of presence and absence measurements. For presence, no additional shielding of the inspected material was added to the model.

For absence measurements additional high-Z material (1.6 cm of lead shielding on all sides) was added to provide a worst-case analysis. HMX was not included in the model for absence of SNM because it would have little impact on the results, i.e., the HE would only modestly attenuate the signal, and the background would be heavily dominated by the Pb shielding. The thickness of $1.6 \mathrm{~cm}$ was chosen to attenuate the passive gamma-ray signature of ${ }^{239} \mathrm{Pu}$ down to $1 \%$. In the case for absence of HE, the HE was placed next to $0.375 \mathrm{~cm}$ of PuAl, which is the material and thickness of Pu-bearing shell in the IO\#8. For diversion detection, the models for absence of SNM were used to detect diversion of SNM, whereas for HE diversion, a $1.6 \mathrm{~cm} \mathrm{~Pb}$ shield was placed around the $\mathrm{HE}$ to provide a worst-case analysis for detection of diversion of $\mathrm{HE}$.

${ }^{2}$ A continuous-wave, or CW, beam is one in which the beam of particles is always on, as opposed to being pulsed, possibly with a long time between relative short pulses. 
Detailed results that apply the NRF signature calculations to quantify capability for each use scenario are presented in the following sections. For treaty verification, one hour is the maximum measurement time that could be considered reasonable, although shorter times would be better. There will be a strong preference to have only one detector, but it is not unreasonable to consider up to four detectors. All of the results shown below are for a single detector.

\section{II.3.3. Simulation Study Setup}

Radiation transport modeling was conducting using the GEANT4 framework $[93,94]$. Both scattering geometries (detecting photons directly scattered from the target) and transmission geometries (detecting photons scattered from a witness foil after passing through the target) [13] were considered for all treaty verification modeling. A single $80 \%$ relative efficiency high-purity germanium (HPGe) detector was used. For scattering, the detector was placed $100 \mathrm{~cm}$ away from the center of the target. For transmission, the detector was placed at $25 \mathrm{~cm}$ away from the center of a witness foil placed downstream of the target and separated from the target by a collimating tungsten shield that allowed the primary beam to pass through the shield but eliminated scattered photons generated in the target. The witness foil was always $1 \mathrm{~cm}$ thick, which provides a best-case evaluation for transmission geometries because of the large efficiency for scattering resonant photons off the witness foil. The MPS was assumed to be operating with a repetition rate of $10 \mathrm{kHz}$ and a $2 \%$ FWHM energy distribution. For MPS beams, a $1 \mathrm{~cm}$ thick lead filter was also placed in front of the detector. A $2.5 \mathrm{~cm}$ thick filter was used for bremsstrahlung beams; the bremsstrahlung beam produces considerably more low energy background events than a MPS beam, so the thicker filter is advantageous.

For transmission measurements, comparisons were made between materials with and without the isotope of interest in the target but maintaining the same overall radiographic transmission. In a transmission measurement, the ratio of the rate of the observed resonant photons from the witness foil with respect to the transmitted beam current is observed. In this modeling, the transmitted beam through the witness foil was not recorded. To provide a directly comparable normalization, the radiographic thickness of the target was held constant while the presence of the resonant isotopes was turned off and on. For instance, transmission measurements on HEU looking for the $1733 \mathrm{keV}$ resonance of ${ }^{235} \mathrm{U}$ were compared against similar geometry, but substituting DU for HEU in the target. This issue does not impact scattering measurements.

For NRF transmission measurements, there is a range of material thickness over which the measurement will perform well. If the material is too thin, then there is not enough resonant absorption in the material to create adequate difference to observe, so high counting statistics are required to differentiate two very similar numbers. As a result, absence measurements using NRF should be performed using scattering techniques. On the other hand, if the material is too thick, whether resonant or non-resonant material, there is not enough flux transmitted through the sample to the monitoring foil, so the detection rates will be slow. Scattering measurements would be vulnerable to shielding, whereas transmission measurements are only sensitive to shielding to the point that it attenuates the transmitted beam (such attenuation would be detected by the technique).

The modeling was handled in two stages to improve computational efficiency. First, the scattered photons of interest, either off the target or off the witness foil depending on the geometry, were recorded after passing through the lead filter for all azimuthal angles and polar angles between 120 and 140 degrees. The number of photons recorded was then scaled to 
account for the solid angle and intrinsic detector efficiency at the resonant energy to determine the observed signal rate.

The intrinsic detector efficiency was determined through a separate GEANT4-based simulation of the detector. An intrinsic total efficiency of $70 \%$ was used to estimate the detector rate. This value was estimated by weighing the total efficiency as a function of energy; the total efficiency was roughly constant for all energies.

In addition to the NRF signal rate, the total detector rate was estimated in the simulations. The number of photons per shot for the MPS measurements was limited so that the probability of pileup was $10 \%$. Pileup occurs when two photons from uncorrelated scattering events are observed by the detector at the same time. When pileup occurs, the information from an NRF event is lost because the event is shifted above the NRF peak. Following Poisson statistics, this requirement limits the mean total detector rate to 0.53 events per shot. The photons per shot was determined based on this mean total detector rate.

The maximum beam current of bremsstrahlung measurements is critical for setting the detector rate. While the number of photons per energy per solid angle per micro-coulomb of beam current was determined through radiation transport modeling using Geant4, the level of beam current was set by empirical experience from measurements on approximately $1 \mathrm{~mm}$ of HEU. The empirical observations on maximum beam current were scaled to account for differences in detector size and detector distance from the target. Maximum electron beam currents for bremsstrahlung measurements of $40 \mu \mathrm{A}$ and $160 \mu \mathrm{A}$ were selected for scattering and transmission measurements, respectively. These currents will provide a $10-20 \%$ pileup probability.

The criterion for detection was determined through the following hypothesis-testing approach. Similar but different approaches were used for the scattering and transmission geometries. For scattering, the required number of counts was determined by

$$
n_{\text {scat }}=\left(\frac{\gamma}{\gamma-1}\right)\left[A_{D}+\frac{1}{\sqrt{\gamma}} A_{F A}\right]^{2},
$$

where $\gamma=\frac{S}{N}+1, \mathrm{~S} / \mathrm{N}$ is the signal-to-noise (SNR) of the NRF signature, and $A_{D}$ and $A_{F A}$ are the number of sigmas required to achieve the desired probability of detection and probability of false alarm, respectively. The number of counts required for a transmission measurement when the material of interest is in the target is given by

$$
n_{\text {tran }}=\beta\left(\frac{1}{1-\beta}\right)^{2}\left[\sqrt{1+\alpha} A_{F A}+\sqrt{\alpha+\beta} A_{D}\right]^{2}
$$

where $\alpha=N_{0} / S_{0}$ is the inverse of the SNR when the material of interest is not in the target, and $\beta=S_{1} / S_{0}$ is the ratio of the resonance signals when the material of interest is in the target to when it is not in the target.

It was necessary to estimate the signal-to-noise ratio for a variety of measurements. The estimates were based on three empirical observations. First, that the signal-to-noise ratio (SNR) of the 1733-keV resonance had a SNR of 1 for bremsstrahlung measurements of $2 \mathrm{~mm}$ of HEU [8]. Second, that measurements at HIGS suggest that a 4.3\% FWHM quasi-monoenergetic photon beam gave a SNR ratio roughly 2.7 times larger on DU than a similar measurement using a bremsstrahlung photon beam, see Figure 35. Correcting for the 2\% FWHM MPS beam under evaluation, the SNR for ${ }^{235 \mathrm{U}}$ was then assumed to be 5.8. Third, the strength of the background, as measured in counts per mole per $\mathrm{cm}^{2}$ per source intensity, scales as the cube of the atomic charge number, i.e. $Z^{3}$ [11]. The SNR for a specific geometry was then determined based on these observations, coupled with a calculation of $C_{\text {thick, }}$, which is a measure of the NRF signal strength as 
described in [8]. One of the consequences of the $Z$ scaling of the continuum background is that the addition of low-Z material, such as HE, has minimal impact on the SNR when SNM is present.

\section{NRF DU}

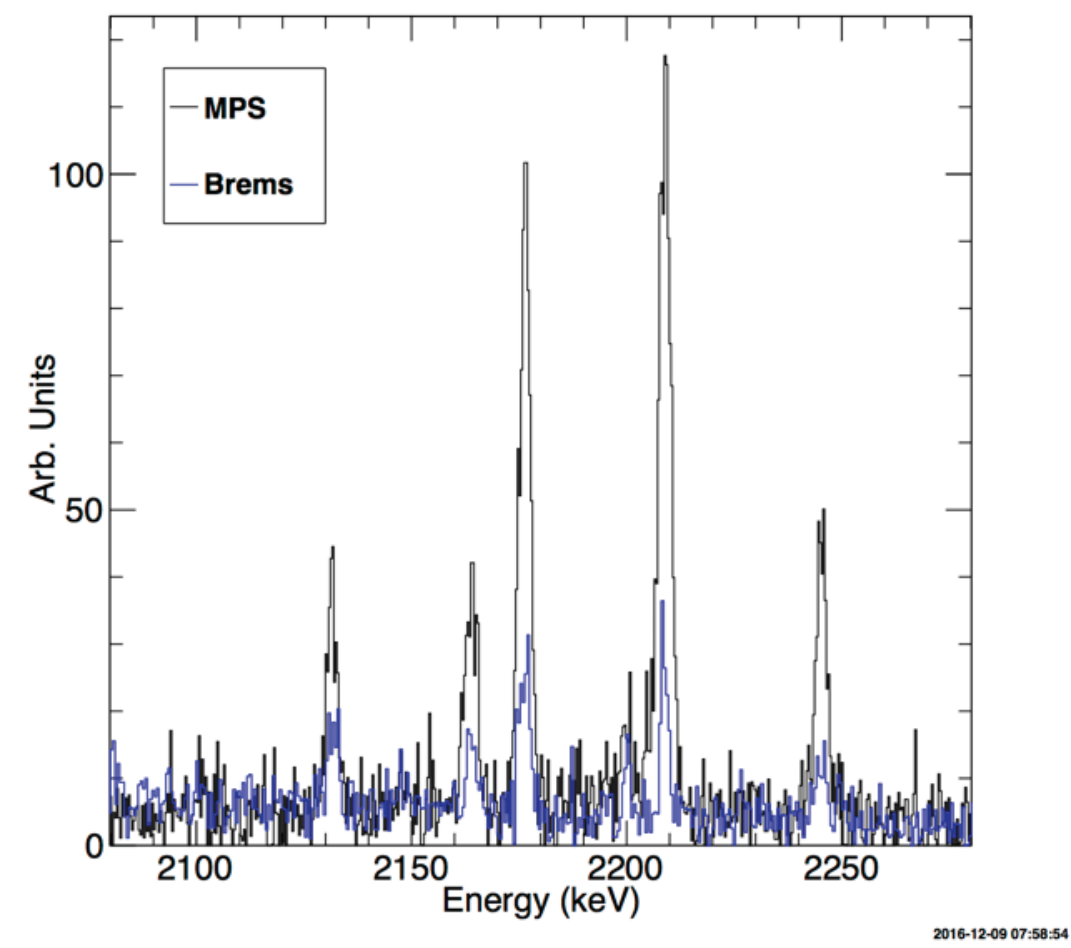

Figure 35. Comparison of NRF response from ${ }^{238} \mathrm{U}$ for $4.3 \%$ FWHM monoenergetic beam at HIGS (MPS) and 2.8 $\mathrm{MeV}$ end-point energy bremsstrahlung source (Brems).

The SNR impacts the number of photons required to declare detection, and the impact decreases significantly once the SNR is greater than 1. For instance, for an NRF scattering measurement with $99 \%$ probability of detection and 1\% probability of false alarm, a SNR of 0.1 requires 228 events to declare detection, an SNR of 1 requires 31 events, and a SNR of 10 requires only 11 events. As a result, the time-to-detection is not too sensitive to the SNR so long as the SNR $>1$.

A summary of the relevant NRF resonance properties is shown in Table 23. The table includes the resonant energy, the integrated cross section, and the bremsstrahlung end-point energy used in the simulations for that resonance. The maximum photons/shot required for scattering measurements is $3 \cdot 10^{5}$, for IO\# 8 .

There are two other critical assumptions made for these calculations. First, it was assumed that the material of interest had been localized, so that the photon beam was always hitting the material of interest in the target. This localization could be accomplished in a number of waysfor instance, through a fast series of transmission measurements. The second assumption was that the beam was scanned over the area of interest. This second assumption is not critical from a time-to-detection perspective, since it is assumed that the material geometry is the same over the scanned area, but is important from a system design perspective in that rastering or scanning the beam over the appropriate area is required. 
Table 23: Properties of NRF resonances. $I_{0}$ is the integrated NRF cross section.

\begin{tabular}{|c|c|c|c|c|}
\hline & $235 \mathrm{U}$ & ${ }^{239} \mathrm{Pu}$ & ${ }^{12} \mathrm{C}$ & ${ }^{14} \mathrm{~N}$ \\
\hline$E_{\text {res }}[\mathrm{MeV}]$ & 1.733 & 2.143 & 4.485 & 4.915 \\
\hline$I_{0}[\mathrm{ev} \mathrm{b}]$ & 29.8 & 13 & 10.5 & 4.4 \\
\hline$E_{\text {brems }}[\mathrm{MeV}]$ & 2.1 & 2.5 & 5.1 & N/A \\
\hline
\end{tabular}

\section{II.3.4. Warhead Confirmation}

\section{II.3.4.1. Background}

One of the most challenging issues in Treaty Verification is the characterization of the full weapon. In order to receive credit for warhead dismantlement, it is necessary to first confirm that the initial object was a nuclear warhead. The detailed definition of the warhead will likely vary with the treaty, as specifics are likely to depend on the type of warhead. A possible definition for a warhead might be:

- Contains at least some threshold of high explosives (HE).

- Contains at least some threshold of fissile material (FM).

- The HE surrounds the FM.

The mass thresholds are likely to be set well below the mass of the actual item to ensure a very high probability of detection to avoid determining the actual mass through a statistical sampling of many warheads. For this study, only the presence of HE and FM were evaluated. The determination of geometric properties of a TAI, which may significantly improve confidence, is often viewed as too intrusive, leading to the risk of releasing classified design information. For that reason, it was not evaluated here.

Measurements on warheads are significantly constrained for safety reasons. Some of the basic concerns that will impact equipment development are:

- Proximity of equipment to warhead

- Ability to continuously observe warhead

- Limitation on flammable materials in the room

- Limitation on batteries in the room

- Limitations on orientation of the warhead

This list is but a small part of the design constraints that must be addressed $[86,91]$.

There is no accepted approach for conducting warhead confirmation in a treaty verification regime. The R\&D system most technically capable of warhead confirmation, the ORNL Nuclear Materials Identification System (NMIS), $[95,96]$ is likely to be too complex and too intrusive to be accepted for use in a treaty verification regime. NMIS uses a combination of neutron transmission radiography and neutron-induced fission mapping to image the object.

There are a number of other techniques that might be useful, but are not fully developed. Passive coincidence or multiplicity counting can determine plutonium mass $[97,98,99]$, but the sensitivity to the HE is unknown. Passive gamma spectroscopy can detect the presence of FM[9], and possibly HE through downscattering, but it is difficult to extrapolate from a single measurement to the mass of FM. Of the possible active interrogation approaches, neutron-based methods are most developed [100]. 
At this time, there is no known radiological or active interrogation technique for differentiating real HE from mock HE, which has the same density and elemental ratios, but is not explosive. Hence, when we refer to "detection" of HE, we refer to detection of a material with the elemental composition of HE.

\section{II.3.4.2. Approach}

Our approach to evaluate the feasibility of MPS-based sources to confirm that a TAI is a warhead is to conduct radiation transport modeling studies, examining the Idaho National Laboratory Inspection Objects (INL IOs). [89] The INL Inspection Objects offer a range of material characteristics to test the capabilities of our measurement setup. If we can successfully determine the challenging characteristics of the INL inspection objects, we may have a greater confidence that the system will also work for actual or purported weapons and/or weapon components. Three different IOs were studied: IO \#5, IO\# 8 and IO\# 10. A summary of the geometries for these IOs is shown in Table 24. Only one material was studied for each geometry: IO \#5 Pu, IO \#8 HEU, IO \#10 polyethylene. The carbon in the polyethylene was used as a surrogate for nitrogen in HE for warhead confirmation. The NRF response from carbon will be approximately twice as strong as that for nitrogen. However, given the short detection times determined, this factor of two is of little practical importance.

Table 24: Summary of geometries for INL IOs used in this study. The layers are numbered in increasing order, going from the outside to the inside of the IO. All thicknesses are in $\mathrm{cm}$.

\begin{tabular}{|c|cc|cc|cc|}
\hline Layer & \multicolumn{2}{|c|}{ IO\# 8 } & \multicolumn{2}{c|}{ IO \#5 } & \multicolumn{2}{c|}{ IO \#10 } \\
& Material & Thickness & Material & Thickness & Material & Thickness \\
\hline 1 & Al & 0.500 & Al & 0.500 & Al & 0.500 \\
2 & Air & 2.500 & LiH & 2.000 & Air & 0.625 \\
3 & DU & 0.250 & Air & 0.500 & Poly & 2.125 \\
4 & Air & 0.250 & DU & 0.375 & DU & 0.250 \\
5 & PuAl & 0.375 & Air & 0.125 & PuAl & 0.375 \\
6 & Air & 0.625 & HEU & 1.000 & Air & 0.625 \\
\hline
\end{tabular}

\section{II.3.4.3. Results}

A summary of the results for the time-to-detection in warhead confirmation for MPS and bremsstrahlung-based measurements, using both scattering and transmission techniques, is shown in Table 25. All times are for use of a single detector. All of the MPS-based scattering measurements require less than one hour, the maximum time considered for a treaty verification measurement. The MPS-based transmission measurements for HEU and HE require less than an hour, but the measurement for WGPu requires almost three hours because of the relatively small amount of WGPu in the beam. The bremsstrahlung measurements for SNM require considerably more time; however the bremsstrahlung measurements for HE require only a few minutes. Furthermore, scattering measurements are faster than transmission measurements for Pu and HE, but not for HEU. That is because there is significantly more HEU in the IO\#5 than Pu in IO\#8, so that the attenuation of the resonant photons in the target is significant enough to quickly distinguish whether the HEU is present. Provided the appropriate measurement is selected, the advantage of MPS-based techniques is roughly two orders of magnitude faster detection for FM, while there is not a significant advantage for carbon. 
Table 25: Time-to-detection for warhead confirmation, and critical parameters.

\begin{tabular}{|c|c|c|c|}
\hline IO & \#5 & \#8 & \#10 \\
\hline Isotope & $235 \mathrm{U}$ & ${ }^{239} \mathrm{Pu}$ & ${ }^{12} \mathrm{C}$ \\
\hline$E_{R}[\mathrm{MeV}]$ & 1.733 & 2.143 & 4.438 \\
\hline $\mathrm{SNR}_{\mathrm{MPS}}$ & 5.8 & 2.5 & 3.6 \\
\hline$\beta$ & 0.099 & 0.84 & 0.48 \\
\hline \multicolumn{4}{|c|}{ Time-to-Detection [min] } \\
\hline MPS-scattering & 8 & 13 & 1 \\
\hline MPS-transmission & 3 & 160 & 2 \\
\hline Brems-scattering & 640 & 940 & 3 \\
\hline Brems-transmission & 690 & 530 & 2 \\
\hline
\end{tabular}

\section{II.3.5. Warhead Dismantlement Confirmation}

\section{II.3.4.1. Background}

Confirmation of warhead dismantlement will likely depend on the treaty. One basic definition of "dismantlement" is the separation of HE and FM. To confirm dismantlement, it is necessary to determine that, for containers that are declared to contain dismantled SNM, no HE is present, while for containers that contain HE, no SNM is present. As a result, for each container it is necessary to perform both a presence-confirmation measurement and an absence-confirmation measurement.

It is important to recognize that the measurement for absence of a material is not the logical inverse of detecting its presence, if for no other reason than the threshold of detection for the two processes will likely be different. Beyond the threshold difference, it is also possible that techniques to determine absence may be significantly different from those to determine presence.

Shielding presents a problem for absence measurements. Adequate shielding will be able to defeat any passive or active interrogation approach designed to detect the absence of a material. In other words, it will be possible to hide material inside shielding such that a measurement approach is fooled into a determination that the material is not present. Such thick shielding can be detected by a transmission measurement and flagged as being too thick to penetrate. We will assume that shielding will be limited to $1 \mathrm{~cm}$ of lead in the warhead dismantlement confirmation measurements for detecting absence of materials. There will likely be a practical limit on the amount of shielding one can place in a container to hide material, due to difficulties in handling heavy containers. For example, $1 \mathrm{~cm}$ of lead lined inside a 55 gallon drum would weight approximately $240 \mathrm{~kg}$. Thicker shielding is addressed in the other sections of this report on cargo screening and detection.

When a presence measurement occurs in dismantlement, the monitor is verifying that the material the host has declared is inside the container. The host will want a presence measurement to pass, so we do not assume that there is any additional shielding added for presence measurements.

There is no accepted approach for conducting warhead dismantlement confirmation in a treaty verification regime. For presence and absence of $F M$, viable R\&D concepts include bremsstrahlung-based radiography, passive gamma spectroscopy measurements, passive neutron counters, and neutron-induced fission. For presence and absence of HE, the neutron 
capture and inelastic neutron scattering are likely the most sensitive for detecting the elements in explosives [101].

\section{II.3.5.2. Approach}

Several different geometries were modeled to evaluate the sensitive to confirming presence and absence of materials. For presence measurements, bare plates of the declared material are evaluated; there is no additional shielding or other material present. The thickness of the plates is varied to understand the time-to-detection as a function of material thickness. For absence measurement additional high-Z material was added. For the absence of SNM, $1.6 \mathrm{~cm}$ of lead shielding on all sides was added. HMX was not included in the model for absence of SNM because it would have little impact on the results, i.e., the HE would only modestly attenuate the signal and the background would be heavily dominated by the Pb shielding. In the case of absence of HE, the $\mathrm{HE}$ was placed next to $0.375 \mathrm{~cm}$ of PuAl, which is the material and thickness of Pu-bearing shell in the IO\#8. The criterion for absence measurements is defined in terms of the time to detect a small amount of shielded material.

\section{II.3.5.3. Results}

The time to confirm the presence of SNM for the dismantlement confirmation objective is shown in Figure 36. Results for both NRF scattering and transmission approaches are shown, as well a result for HEU scattering using a bremsstrahlung beam for comparison. NRF scattering using an MPS can confirm the presence of SNM down to $0.1 \mathrm{~mm}$ thickness in less than 4 minutes. Transmission measurements take considerably longer. Even at $10 \mathrm{~mm}$ thickness, the time to confirm the presence of WGPu using transmission is longer than 90 minutes, so these points are not visible in the selected vertical scale. Thus, scattering measurements prove to be more sensitive. The time to confirmation of HEU using NRF scattering for a bremsstrahlung beam is also shown. This result is fairly typical, with the time to confirmation being an order of magnitude larger than with an MPS-based approach. The maximum MPS photons/shot required for scattering is $1 \times 10^{6}$, for $0.1 \mathrm{~cm}$ WGPu. The results for scattering off SNM using an MPS show the curious result of reduced times to detection as the materials get thinner. The behavior is created by the limitation on photons/shot, which stems from limits on the detector rates. The thinner the material, the higher the photons/shot that can be used to maintain the same level of pileup, which reduces the time to detection. 


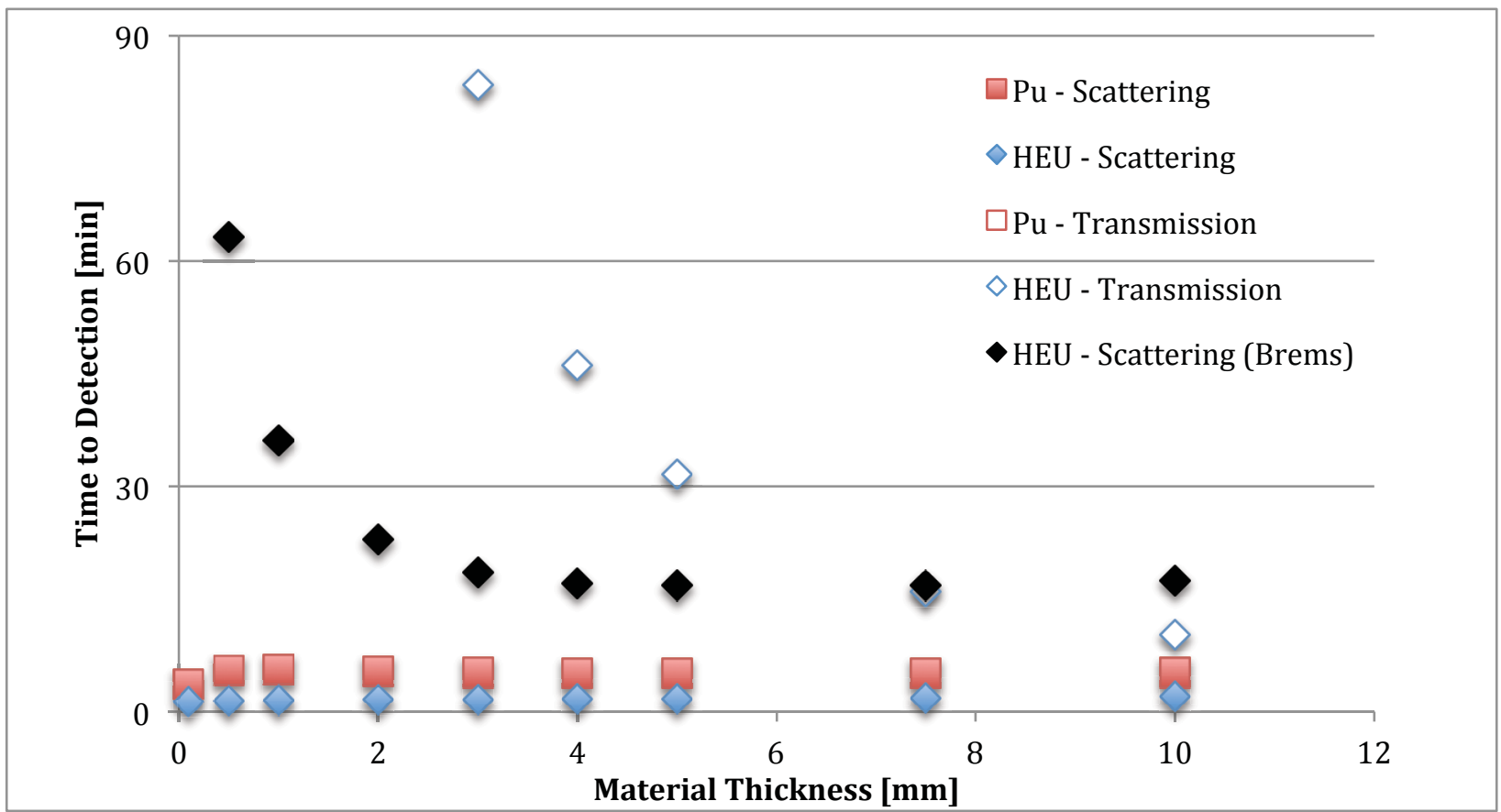

Figure 36. Time to confirm presence of SNM for dismantlement confirmation. None of the Pu transmission time-to-detection confirmations took less than 90 minutes.

The times to confirm the presence of HMX for the dismantlement confirmation objective are shown in Figure 37. Only results for scattering are shown; the sensitivity in the transmission measurements was very poor, leading to time-to-detection greater than 1,000 minutes. Again, scattering outperforms transmission, and provides the ability to confirm the presence of HMX down to $0.5 \mathrm{~cm}$ thick in less than 10 minutes. The maximum MPS photons/shot required for scattering is $1 \times 10^{6}$, for $0.5 \mathrm{~cm} \mathrm{HMX}$.

The time to detect hidden SNM during confirmation-of-absence measurements in the challenging geometry is shown in Figure 38. Again, scattering measurements outperform transmission measurements. Interpolating the curves, $1.3 \mathrm{~mm}$ of HEU and $3.4 \mathrm{~mm}$ of WGPu can be detected in an hour. Transmission measurements begin to outperform scattering measurements for HEU measurement for HEU thicknesses of $7 \mathrm{~mm}$ or greater. The time-todetection for Pu using transmission was greater than 90 minutes for all thicknesses studied; at $10 \mathrm{~mm} \mathrm{WGPu}$, the time-to-detection was 140 minutes. The maximum photons/shot required is $1 \cdot 10^{5}$, for $0.1 \mathrm{~cm}$ HEU. The differences between Figure 36 and Figure 38 indicate the impact of the lead shielding; without the lead shielding, the ability to detect smaller amounts of SNM would follow the results from Figure 36. 


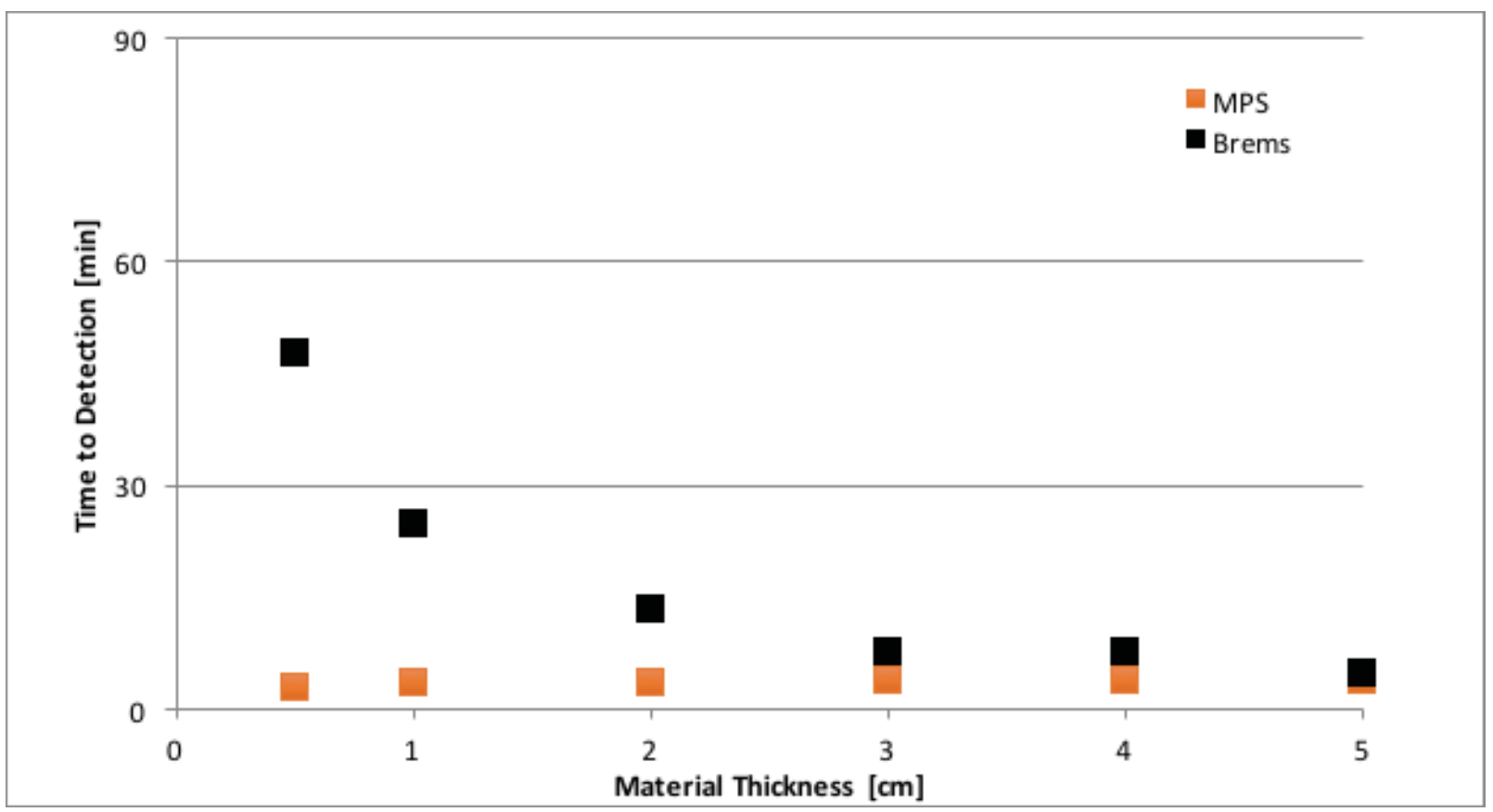

Figure 37. Time to confirm presence of HMX using NRF scattering for dismantlement confirmation. Note that the transmission response was very poor, leading to large (greater than 1000 minutes) times to detection.

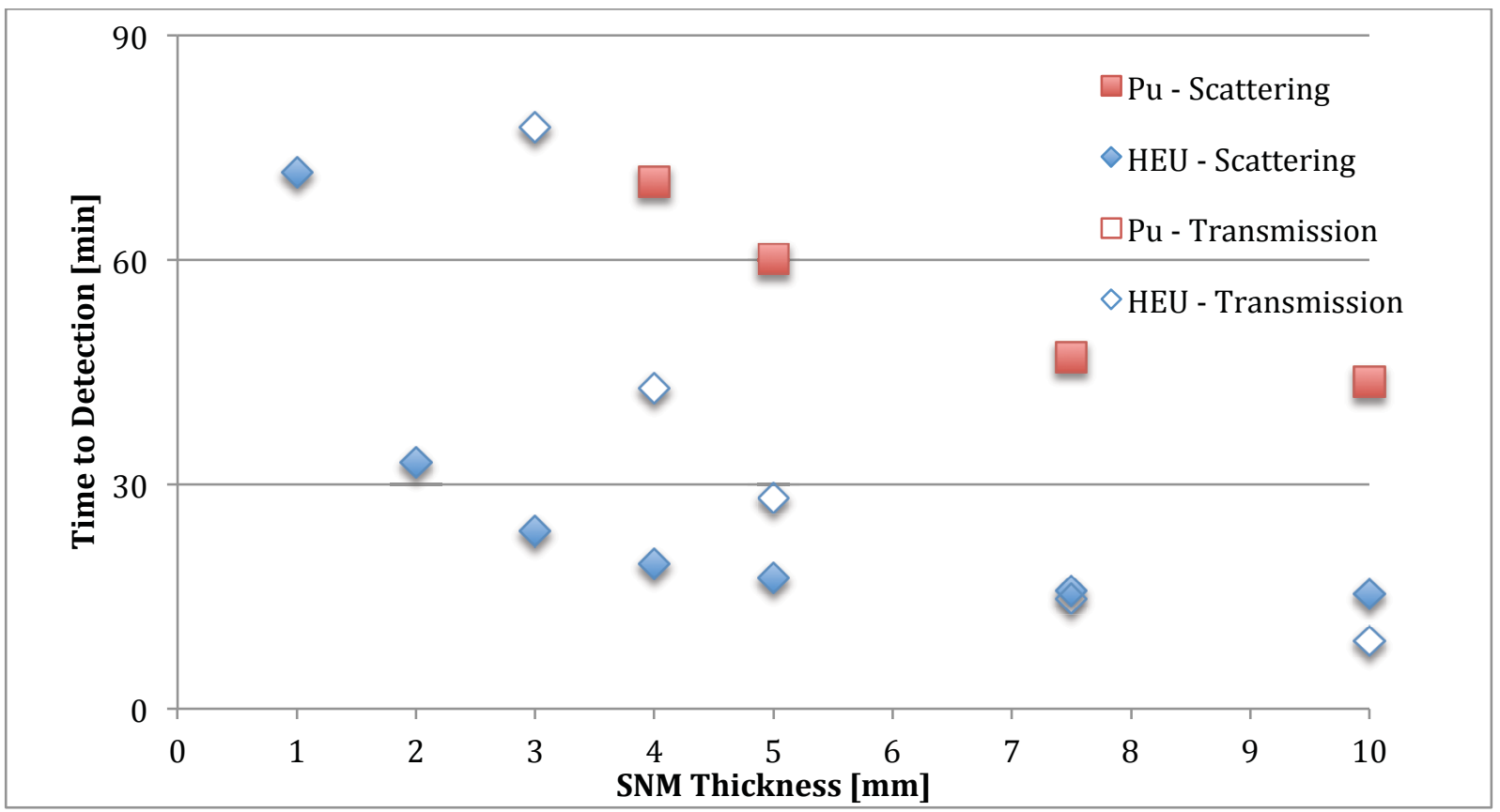

Figure 38. Time to detect SNM for absence measurements. Pu transmission measurements up to $10 \mathrm{~mm} \mathrm{Pu}$ take more than 90 minutes.

The time to detect hidden HMX during confirmation of absence measurements is shown in Figure 39. Only results for scattering are shown, as the transmission results were greater than 1000 minutes. Note that the observation of ${ }^{14} \mathrm{~N}$ in HMX is used as the indicator for HE detection. If other nitrogen-bearing materials are present in the warhead, additional measurements looking at other isotopes (e.g., of carbon and oxygen) might help to reduce the probability of false positives. The maximum MPS photons/shot required is $6 \times 10^{4}$, for $1-\mathrm{cm}$ HMX. 
The time to detect hidden HMX during confirmation of absence measurements is shown in Figure 39. Only results for scattering are shown, as the transmission results were greater than 1000 minutes. Note that the observation of ${ }^{14} \mathrm{~N}$ in HMX is used as the indicator for HE detection. If other nitrogen-bearing materials are present in the warhead, additional measurements looking at other isotopes (e.g., of carbon and oxygen) might help to reduce the probability of false positives. The maximum MPS photons/shot required is $6 \times 10^{4}$, for 1 -cm HMX.

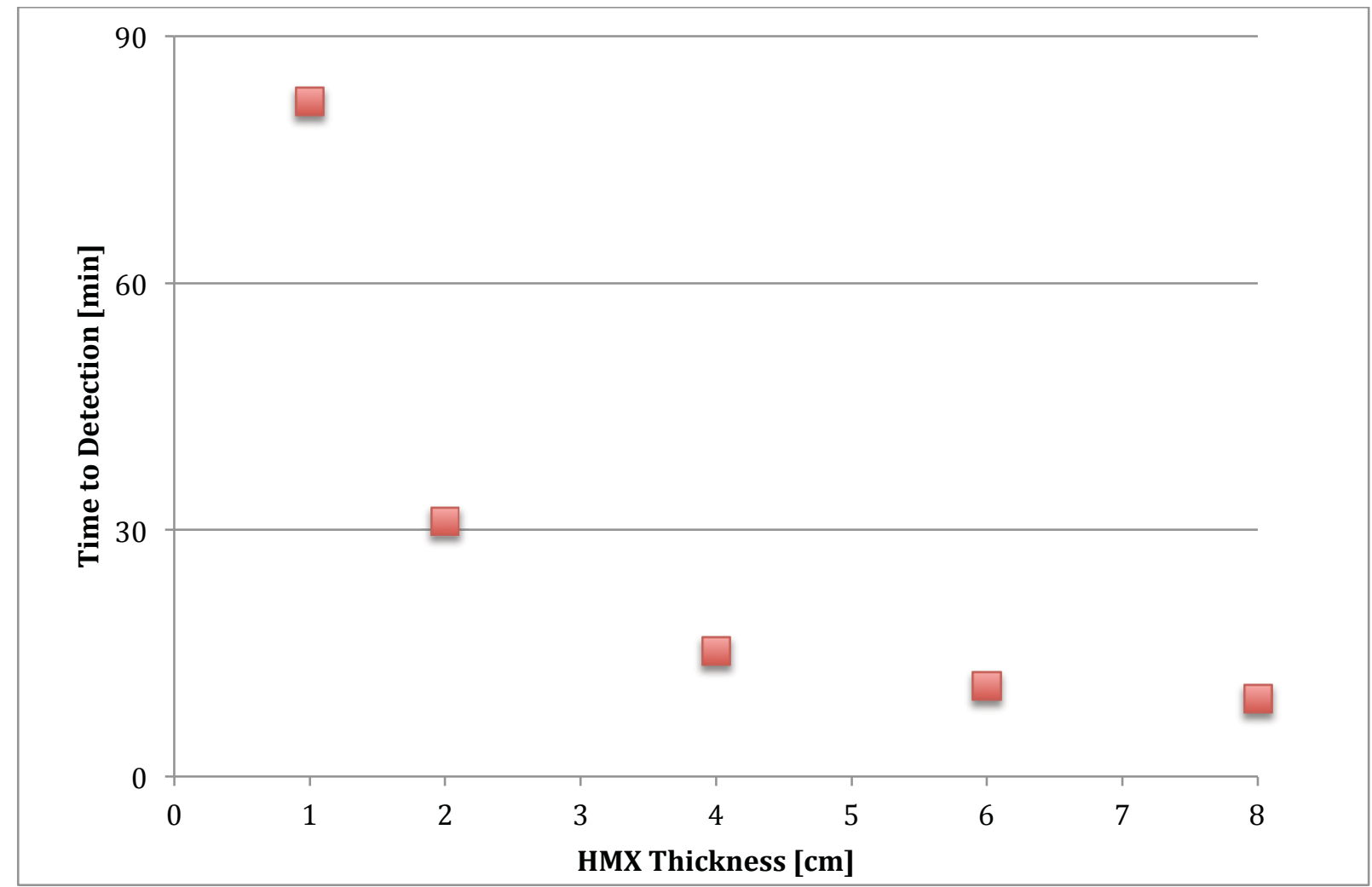

Figure 39. Time to detect HMX for absence measurements using NRF scattering. The time to detect for transmission measurements is not shown because all results exceeded 1000 minutes.

\section{II.3.6. Materials Diversion Detection}

\section{II.3.6.1. Background}

The basic process for dismantlement is that one warhead, people, tools and empty containers enter a room, and over multiple days the warhead is disassembled and placed into various containers. Anything leaving the room is a possible vector for material diversion. The focus of diversion is on FM, but there may be interest in also detecting HE diversion. Diversion by people carrying material can be monitored with portal monitors. The containers and tools may be subject to inspection. Some of the containers should contain only HE, and some should contain only FM, while others may contain various non-treaty-limited items and tools (some tools may be considered sensitive). Possible measurement approaches are:

- Absence of HE: NRF scattering or transmission measurement to detect lack of nitrogen in the sample.

- Absence of FM: photofission, NRF scattering or transmission. 


\section{II.3.6.2. Approach}

The geometries considered for diversion detection consisted of $1.6 \mathrm{~cm}$ of $\mathrm{Pb}$ shielding surrounding the material of interest. This geometry has already been evaluated for SNM detection; see Figure 38. The geometry for HE detection in a diversion scenario has not been previously evaluated.

\section{II.3.6.3. Results}

Figure 38 shows the equivalent results for SNM detection in the diversion detection scenario. NRF scattering can confirm the presence of SNM down to $0.5 \mathrm{~mm}$ thickness in a little more than an hour using a single detector. Transmission measurements take considerably longer; even at 10 $\mathrm{mm}$ thick, the time to detect WGPu is longer than 90 minutes. Thus, scattering measurements prove to be more sensitive.

The time to detect HE in the diversion detection case is shown in Figure 40. HMX was used for the HE. Only results for NRF scattering are shown, because based on experience with dismantlement confirmation, the transmission approach is not sensitive enough to warrant consideration. Results for both MPS-based and bremsstrahlung-based photon sources are considered. Thicknesses down to $1 \mathrm{~cm}$ can be detected in less than 35 minutes using NRF scattering techniques with an MPS-based source; a bremsstrahlung-based source would require approximately an order of magnitude longer. The maximum MPS photons/shot required is $1 \times 10^{5}$, for $1 \mathrm{~cm}$ HMX thickness.

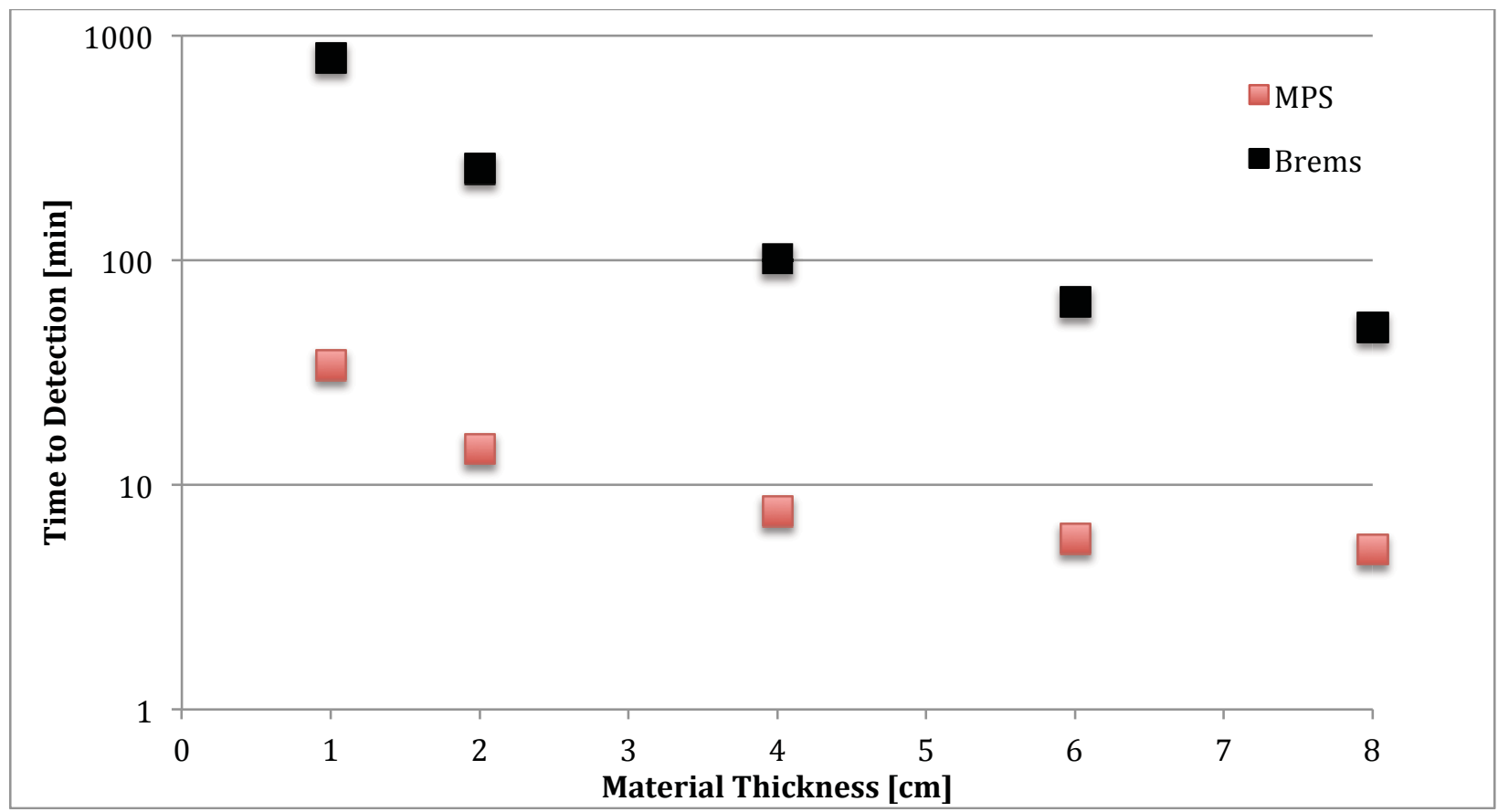

Figure 40. Time to detect HE in diversion detection scenario. Only results for scattering, both MPS and bremsstrahlung, are shown

\section{II.3.7. Impact of and Requirements for MPS Systems}

The potential impact of MPS for treaty verification measurements could be very significant. An MPS could enable warhead confirmation, for which there is currently no known solution. In 
addition, a system suitable for warhead confirmation could likely be used for dismantlement confirmation, which would reduce the number of instruments required for treaty verification. In addition, a suitable MPS system may enable a physical information barrier to restrict knowledge to specific isotopes (e.g., it could be sensitive to ${ }^{239} \mathrm{Pu}$ but not ${ }^{240} \mathrm{Pu}$ ). As described in the next section, an MPS could also enable a unique identifier for warheads and components through highresolution radiography.

Material characteristics and their effect on measurement setup in the context of treaty verification objectives of warhead confirmation, dismantlement confirmation and diversion detection have been evaluated considering both NRF scattering and transmission techniques, using both MPS-based and bremsstrahlung-based photon sources. A summary of the results is presented in Table 26.

Table 26: Summary of results for Treaty Verification results. For Warhead Confirmation, the time to detect the material is listed, whereas for the other tests the minimum thickness of material that can be detected with a single $80 \%$ relative efficiency HPGe detector in one hour is shown. Thicknesses denoted with an asterisk are the smallest thicknesses evaluated and are not limited by the measurement time.

\begin{tabular}{|l|l|l|l|l|l|}
\hline Objective & Test & Geometry & $\begin{array}{l}\text { Time/ } \\
\text { Thickness }\end{array}$ & $\begin{array}{l}\text { NRF } \\
\text { Technique }\end{array}$ & $\gamma /$ shot \\
\hline \multirow{4}{*}{$\begin{array}{l}\text { Warhead } \\
\text { Confirmation }\end{array}$} & HEU Detection & IO\#5 & $3 \mathrm{~min}$ & Transmission & $2 \cdot 10^{6}$ \\
\cline { 2 - 6 } & Pu Detection & IO\#8 & $13 \mathrm{~min}$ & Scattering & $3 \cdot 10^{5}$ \\
\cline { 2 - 6 } & HE Detection & IO\#10 & $1 \mathrm{~min}$ & Scattering & $3 \cdot 10^{4}$ \\
\hline \multirow{4}{*}{$\begin{array}{l}\text { Dismantlement } \\
\text { Confirmation }\end{array}$} & Presence HEU & Bare & $0.1 \mathrm{~mm}^{*}$ & Scattering & $1 \cdot 10^{6}$ \\
\cline { 2 - 6 } & Presence Pu & Bare & $0.1 \mathrm{~mm}^{*}$ & Scattering & $1 \cdot 10^{6}$ \\
\cline { 2 - 6 } & Presence HE & Bare & $0.5 \mathrm{~cm}^{*}$ & Scattering & $1 \cdot 10^{6}$ \\
\cline { 2 - 6 } & Absence HEU & $1.6 \mathrm{~cm} \mathrm{~Pb} \mathrm{Shield}$ & $1.4 \mathrm{~mm}$ & Scattering & $9 \cdot 10^{4}$ \\
\cline { 2 - 6 } & Absence Pu & $1.6 \mathrm{~cm} \mathrm{~Pb} \mathrm{Shield}$ & $5 \mathrm{~mm}$ & Scattering & $5 \cdot 10^{4}$ \\
\cline { 2 - 6 } & Absence HE & $3.75 \mathrm{~cm}$ PuAl & $1.5 \mathrm{~cm}$ & Scattering & $6 \cdot 10^{4}$ \\
\hline \multirow{4}{*}{$\begin{array}{l}\text { Diversion } \\
\text { Detection }\end{array}$} & Absence HEU & $1.6 \mathrm{~cm}$ Pb Shield & $1.5 \mathrm{~mm}$ & Scattering & $9 \cdot 10^{4}$ \\
\cline { 2 - 6 } & Absence WGPu & $1.6 \mathrm{~cm}$ Pb Shield & $5.0 \mathrm{~mm}$ & Scattering & $5 \cdot 10^{4}$ \\
\cline { 2 - 6 } & Absence HE & $1.6 \mathrm{~cm}$ Pb Shield & $1 \mathrm{~cm} *$ & Scattering & $1 \cdot 10^{5}$ \\
\hline
\end{tabular}

Several high-level conclusions can be made. MPS-based photon sources provide performance an order of magnitude better (if not more) than bremsstrahlung-based photon sources. Measurements relevant to warhead confirmation would take minutes, and in an hour, detection of mm-scale HE or sub-mm scale FM thicknesses could be accomplished, relevant to dismantlement. In shielded cases for diversion detection, the absence of few-mm-scale thicknesses of FM, or cmscale HE, is verifiable. NRF scattering generally performs better than NRF transmission; the one exception is the test case for the confirmation of HEU in IO\#5, which has relatively thick HEU. The shielding in the absence measurements makes these measurements more demanding; the assumed shielding is likely a worst-case scenario. It is important to note that the time to detection/confirmation is based on a single $80 \%$ relative efficiency HPGe; times could be reduced using additional detectors.

The primary limitation to reducing the time-to-detection/confirmation is the total rate on the detector; if the total rate is too high, pileup will occur, obscuring the signal. Evaluation of different filtering options in front of the detector may improve that situation. Alternatively, if one could 
develop a fast threshold counting detector, it may be possible to significantly reduce the times to detection/confirmation. The photon source is assumed to be operating at $10 \mathrm{kHz}$ with a $2 \%$ FWHM energy distribution, and the photon intensity is modest, at less than $2 \times 10^{6} \gamma /$ shot. Because of the limitation on photon intensity that stems from the need to limit detector rate, the time to detection will be approximately proportional to the width of the energy distribution. For example, a 1\% FWHM would reduce the times to detection by a factor of 2 .

Constraints on dose may be a challenge for NRF measurements of active warheads. The application dose limit was estimated based on a study examining devices in space. According to Johnston, there is little reported damage for $1 \mathrm{krad}$ of dose on silicon for electronics. [102] The $\mathrm{NRF}$ scattering measurement may impart more than this dose by roughly an order of magnitude. Dose requirements may be loosened or removed if the warhead is to be dismantled. If lower dose is a priority, decreasing the energy spread would reduce the dose rate and/or enable lower repetition rates. There are numerous operational constraints that will have to be addressed by any measurement system for treaty verification to address safety and information concerns. If after further detailed examination an MPS approach is viable for treaty verification measurements, it would be worthwhile to conduct a high-level review of the possible impact those constraints might have on a deployed MPS-based measurement system.

The size and transportability of the equipment will be limited by a couple of factors. The size of the equipment will be limited by both the available floor space in the room in which measurements will be performed and the size of the door to access the room. We assume that the equipment will need to be moved into and out of the measurement room on a roughly monthly basis, so that the room is available for other activities. Setup and teardown of the equipment should take less than four hours, though this is not a hard limit. Given the lack of definitive constraints on the dimensions of the room and door, the envisioned 20-foot container size for some candidate MPSs is likely close enough that appropriate size and transportability could be engineered into a system. The system size requirements of measurements on non-HE dismantled materials may be relaxed compared to those for warhead confirmation. For safety reasons, the location of measurements on HE is limited to specific rooms. Measurements on FM and other nonHE materials can in principle be conducted at other locations, which may relax some of the system size requirements. 


\section{II.4. High Resolution Imaging for Stockpile Stewardship}

\section{II.4.1. Introduction}

One of the objectives of stockpile stewardship is evaluating whether the active nuclear weapon inventory remains both effective and safe. To conduct these evaluations, non-destructive analyses are conducted on assembled warheads and components. Technologies for stockpile stewardship may be related to technologies for treaty verification. Any measurement that the US is likely to do as part of a treaty verification will almost certainly have been extensively tested on its own warheads prior to treaty negotiations $[2,103]$. Stockpile stewardship is a natural avenue to gain that experience. The U.S. has an existing capability for conducting 3D imaging of its nuclear warheads. The Confined Large Optical Scintillator Screen and Imaging System (CoLOSSIS), located at Pantex, was designed to conduct 3D computed tomography on warhead and components with the goal of observing features on the scale of 50 to $75 \mu \mathrm{m}$ [104]. In practice, it takes a week to collect the 1500 views that are then combined to form the 3D image. An MPS may be able to improve on this existing system in three ways: 1) achieve higher spatial resolution, 2) reduce measurement time which could increase throughput of inspections, and 3) reduce dose to the warhead. The first two improvements are significant drivers for investigating how an MPSbased system may improve on the existing bremsstrahlung-based capability. Two critical issues must be addressed in this evaluation: the relative intensities of the two systems and the differences in imaging.

An MPS-based photon source for stockpile stewardship may have a few advantages over the existing capability beyond the total photon flux. First, the narrow beam aspect of a Thomson MPSbased photon source may enable the reduction of scattering contributions in the image. For thick materials, scattering can be a significant contribution to the exposure for imaging. While bremsstrahlung beams are sometimes collimated to mimic a narrow angle source, this severely restricts flux. If a bremsstrahlung beam were collimated to match the $0.56 \mathrm{~mm}^{2}$ beam from the MPS discussed here, its flux would be reduced a thousandfold, increasing scan time. Another potential MPS advantage is the elimination of sagging correction for thick materials. The continuous energy spectrum of the bremsstrahlung beam requires a correction to properly reconstruct the 3D image due to the difference in attenuation for the different energies. A nearly monoenergetic photon beam would significantly reduce, if not eliminate, the need for that correction. Third, the small emission spot size of Thomson MPSs beam may enable higher spatial resolution. These possible enhancements motivate a more detailed evaluation of the potential benefit of MPS-based photon sources for stockpile stewardship. Simulations were conducted to evaluate each of these major MPS characteristics and their contributions to potential improvements in imaging.

\section{II.4.2. Problem Definition: Imaging Intensity Requirements}

For imaging measurements, the first challenge is providing enough photons to achieve the necessary performance. Flux in excess of the current capability can be used to improve performance - for instance, either reducing measurement time or increasing spatial resolution.

The major components of CoLOSSIS [104] are the linear accelerator, collimators, the imaging system, and the hardware and software to calculate the image. The linear accelerator provides a 9-MeV endpoint energy bremsstrahlung photon beam, which is collimated and directed onto the target $6.5 \mathrm{~m}$ away. The collimated beam delivers about 50 rads per minute at the target with a square cross sectional area of about $24 \mathrm{~cm}$ per side. The x-rays passing through the target strike a scintillator, which appears to be about $50 \mathrm{~cm}$ downstream of the target, and which converts the $\mathrm{x}$ - 
rays to green light. While the engineering of the imaging system is critical to the performance of CoLOSSIS, for the purpose of this evaluation we will assume similar detector performance for an MPS-based system so that it is not necessary to discuss the existing imaging system.

A comparison to current CoLOSSIS capabilities [104] indicates where a Thomson-based MPS [30] could offer performance advantages. With the given information on CoLOSSIS, we can estimate the photon flux at the target. If one assumes that the average energy of the $9-\mathrm{MeV}$ bremsstrahlung beam is $3 \mathrm{MeV}$, then the fluence-to-dose scale for photons is approximately $10^{-11}$ $\mathrm{Sv} \mathrm{cm}^{2}$ [105]. This implies that $50 \mathrm{rad}$ per minute, which is equivalent to $0.5 \mathrm{~Sv}$ for photons, corresponds to $8.3 \cdot 10^{8}$ photons $/ \mathrm{cm}^{2} / \mathrm{sec}$. If the imaging camera covers the full 24 -cm-per-side square of the beam at the target and the average exposure time is 60 seconds, then the flux is $5.0 \cdot 10^{10}$ photons $/ \mathrm{cm}^{2} /$ minute.

A high-repetition rate, high yield MPS-based photon source can provide similar photon flux density. The narrow-beam nature of the MPS-based source requires a slightly different approach to evaluating the time to achieve the same flux as CoLOSSIS. With a Thomson-based MPS there is a trade-off between energy spread and angle. For example, a $4 \mathrm{MeV}$ MPS photon beam with a $2 \%$ energy spread, $2 \mu \mathrm{m}$ beam emission spot, and $0.42 \mathrm{mrad}$ divergence at $100 \mathrm{~cm}$ will cover an area of $0.56 \mathrm{~mm}^{2}$. For $10^{7}$ photons/shot, at a $200 \mathrm{kHz}$ repetition rate, it will take 28 shots to achieve the same exposure per unit area as a bremsstrahlung beam. As a result, it will take 21 seconds to achieve the same exposure across the entire $24-\mathrm{cm}$ square area, allowing for a $30 \%$ overlap in views. In other words, the described MPS photon source can provide three times the photon fluence at $4 \mathrm{MeV}$ in the same amount of time as the current CoLOSSIS system. Note that the energy spread used here is very narrow, hence the number of photons per shot is conservative relative to what is likely required for radiographic measurements. Other pairs of yield and repetition rate could also be considered at similar performance (e.g., $10^{8}$ photons/shot at $20 \mathrm{kHz}$ ). Even for a lower-repetition-rate MPS that does not deliver such high flux, the other advantages discussed below may be compelling.

\section{II.4.3 Simulation Study Setup}

A monoenergetic photon source may have favorable properties for imaging, and in particular for quantitative imaging techniques such as tomography, which is used for stockpile stewardship. The comparison above indicated that the MPS should be capable of equivalent or even greater usable flux than a 9-MeV linac system currently available. Here, we describe models in MCNP of both a $9-\mathrm{MeV}$ linac system with a bremsstrahlung spectrum, and a potential laser-plasma based Thomson monoenergetic photon source (MPS).

We performed a resolution test to determine whether the smaller emission spot of the MPS is likely to offer the enhanced image resolution predicted by geometric optics. A penetration test was also conducted to estimate relative amounts of beam hardening and scatter between the two sources; these effects interfere with quantitative measurements such as tomographic reconstructions. We find that the MPS offers greatly enhanced spatial resolution compared to the linac, that it eliminates beam hardening, and that (due to the small opening angle) it significantly reduced scatter. All these findings indicate that the MPS should offer higher resolution tomography measurements with reduced artifacts.

Radiation transport modeling was conducted to evaluate the emission spot and penetration tests. An MCNP model was developed to represent high resolution imaging with a linac source; see Figure 41. For the purpose of comparing the potential advantage of bremsstrahlung-based sources and MPSs for high-resolution imaging, a perfect detector has been assumed; the focus is 
on the nature of the photons reaching the detector. Although developing an efficient highresolution detector is a large topic in itself, here we focus on limitations to imaging due to the source only. We model flux using a radiography tally with a length scale chosen to easily represent the object of interest.

The linac system is modeled on a Varian $9 \mathrm{MeV}$ Linatron. The linac has a variable focal spot size; for high-resolution imaging we assume $1 \mathrm{~mm}$ focal spot, although this would require a reduction in the overall flux from the peak rate. This is represented by an area source $1 \mathrm{~mm}$ in diameter from which particles are thrown. A large standoff distance based on approximate CoLOSSIS geometry, $6.5 \mathrm{~m}$ from source to test object, is needed to reduce the geometric blurring from the mm-scale linac spot size. Three tungsten collimators along the beam path limit the contributions due to scatter. A nominal detector is placed $90 \mathrm{~cm}$ behind the test object.
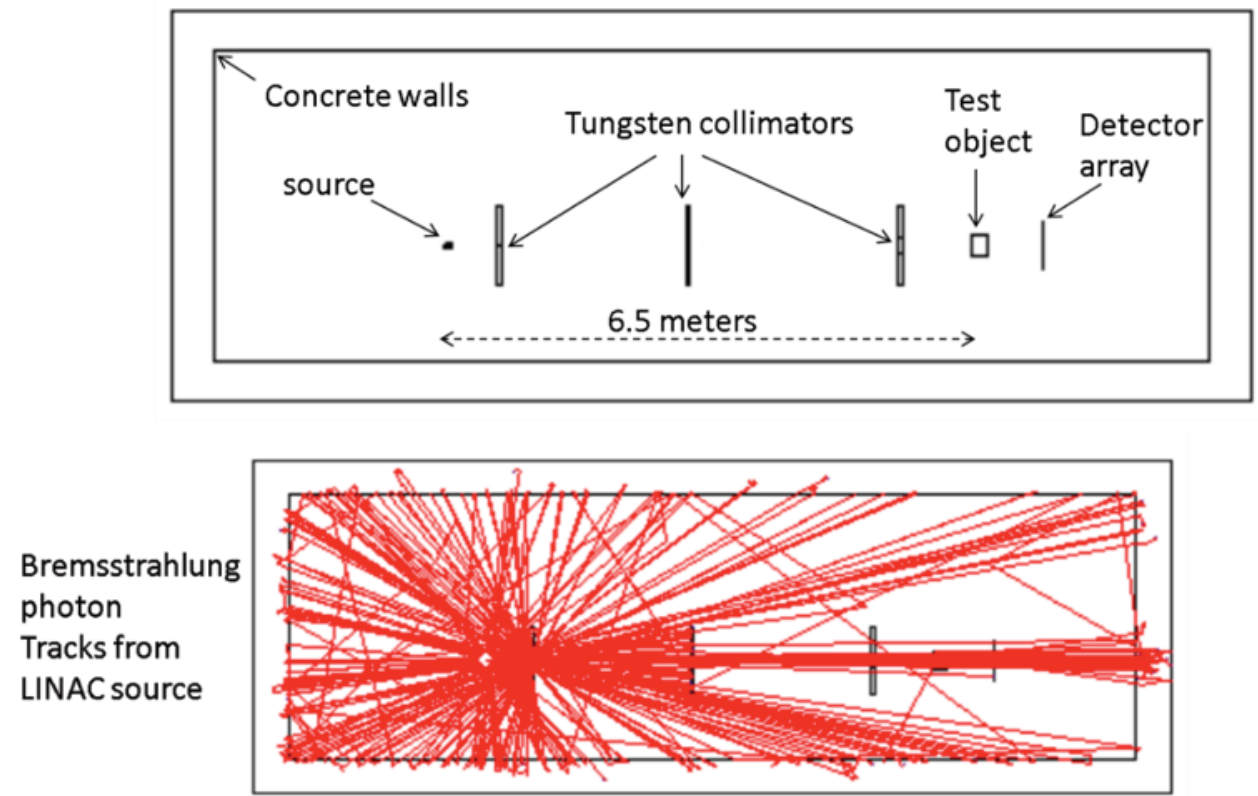

Figure 41. Modeled geometry (top) and example of bremsstrahlung photon tracks from the linac source (bottom).

The MPS source has a distinctive energy-angle dependence, as shown in Figure 42, and a $1 \mu \mathrm{m}$ diameter emission spot [30]. Note that while much smaller than that of the linac, this emission spot size is conservatively large for laser-plasma based Thomson sources. Plasma based beam sizes down to $0.1 \mu \mathrm{m}$ have been measured. The MPS properties were represented in the MCNP model by using a custom SDEF card. No cutoff was used in either energy or radius, although only the central portion of the beam was used in testing. In order to compare the effects of source characteristics on imaging, the MPS was modeled using the same geometry as the linac. The angular divergence of the MPS beam is very small, such that only a few $\mathrm{mm}$ are illuminated even at a distance of $6.5 \mathrm{~m}$. In order to image a large field of view, the beam must be rastered. For the purposes of this investigation, we assume that this could be done. 


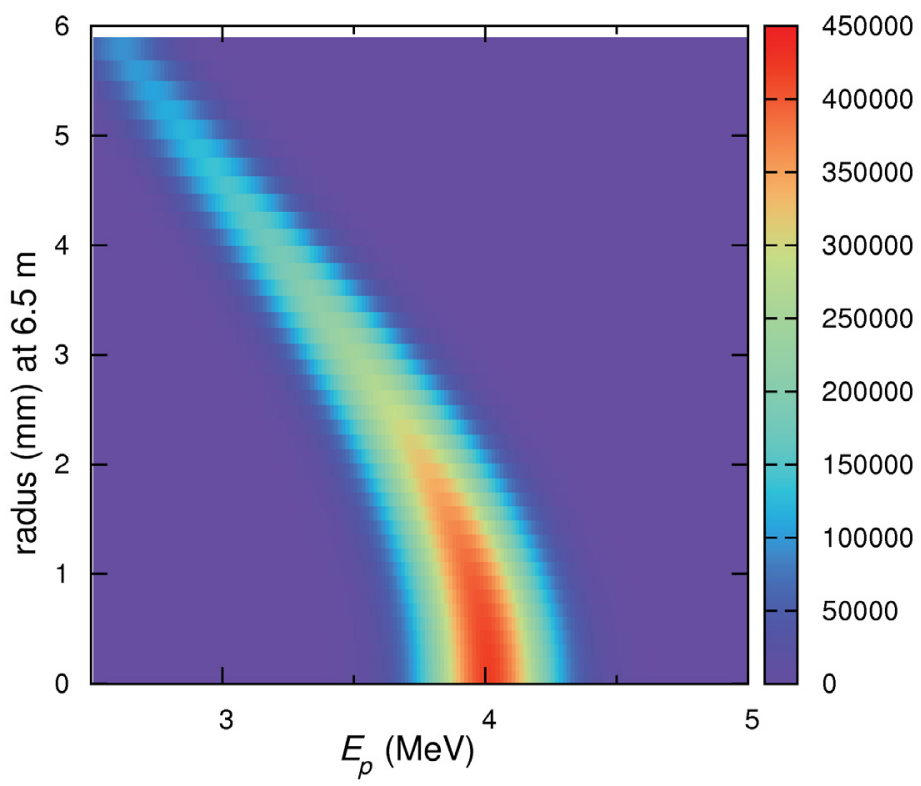

Figure 42. Energy-radius distribution of the Thomson scattered source $6.5 \mathrm{~m}$ downstream, showing the angular dependence of the spectrum.

\section{II.4.4 Simulation Results: Resolution Test}

According to geometric optics, the $1 \mu \mathrm{m}$ effective spot size of the MPS should offer the possibility of greatly enhanced spatial resolution as compared to a linac, which typically has a focal spot size of a few $\mathrm{mm}$ (as little as $1 \mathrm{~mm}$ is achievable, but with decreased flux). However, given the extreme geometry, it is unclear whether this can be expected to hold in the presence of scattering in a real measurement. In a typical imaging setup, resolution can be limited by either the source or the detector. We assume that the detector resolution is arbitrarily fine, and focus on the limitations posed by the source alone.

ANSI N42.46 offers a set of standards for cargo radiography, which is an example of a highenergy imaging application [106]. In this standard, spatial resolution is measured using a set of three steel bars, of width $d$, spacing $d$, and thickness $d$, where $d$ is varied from several cm to mm's. For this application, we are interested in resolution well below $1 \mathrm{~mm}$. For bar thicknesses below $1 \mathrm{~mm}$, the attenuation is negligible, so we fix thickness at $1 \mathrm{~mm}$ and use tungsten as the material. As seen in Figure 43, $200 \mu \mathrm{m}$ features are clearly resolved with a $1 \mathrm{~mm}$ focal spot on the $9 \mathrm{MV}$ linac, but $100 \mu \mathrm{m}$ is more challenging. Anything below about $60 \mu \mathrm{m}$ is not resolved. Using the same geometry with the MPS, as shown in Figure 44, a $1 \mu \mathrm{m}$ feature size is clearly resolved. In this case the $6.5 \mathrm{~m}$ standoff distance is unnecessary, but was used for consistency. By reducing standoff, a MPS source in this case could enable a more compact setup in addition to higher resolution. 


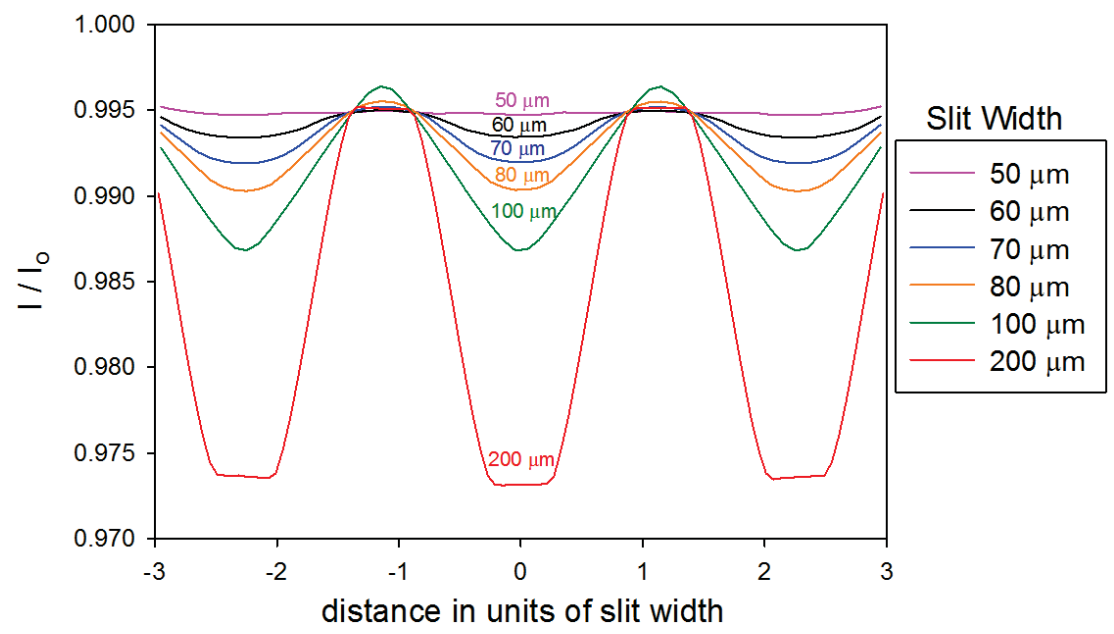

Figure 43. Normalized intensity profile for the linac system across a 3-bar resolution test object with varying dimension d. Bar depth is $1 \mathrm{~mm}$ in all cases.

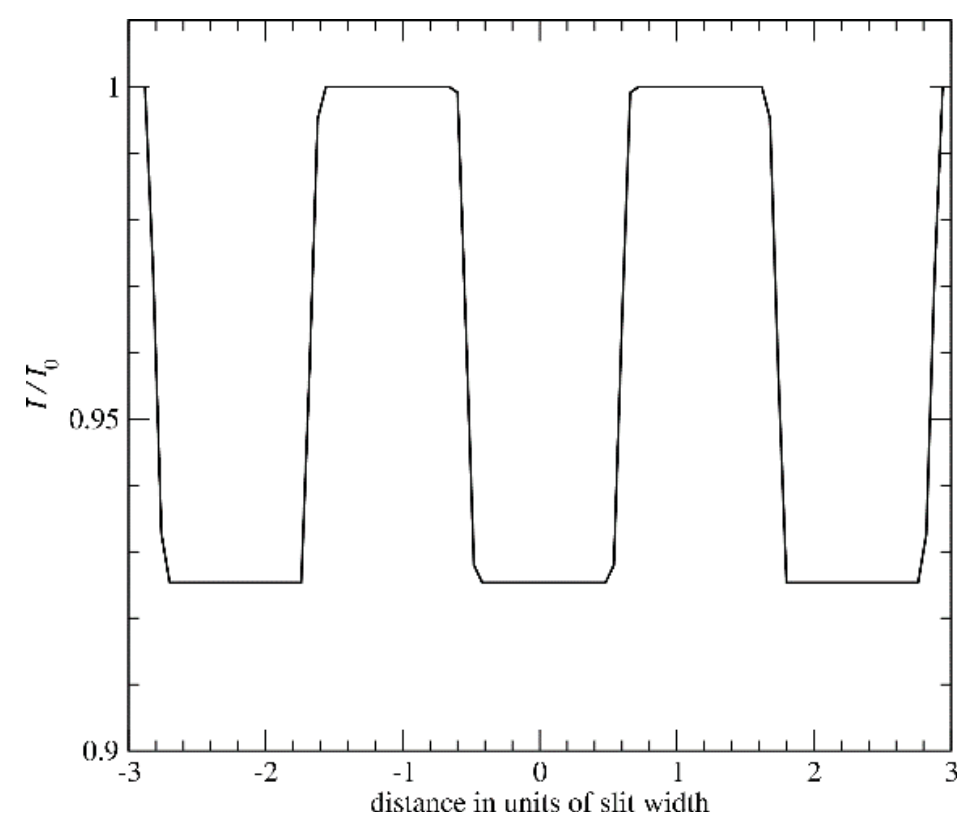

Figure 44. Normalized intensity profile for the MPS system across a 3-bar resolution test object with slit width $1 \mu \mathrm{m}$ and depth $1 \mathrm{~mm}$.

\section{II.4.5 Simulation Results: Penetration test}

For the penetration test, we examined the relative intensity as a function of thickness behind a solid tungsten block that covers approximately half the field of view. This is an imperfect measure of scatter, as the amount of scatter depends on the details of the environment. However, by holding the environment constant while varying the source definition, we are able to compare the performance of the two sources. In medical imaging, scatter of a system is typically defined as the limiting value as the radius of a thick object is reduced to zero [107].

Figure 45 shows relative intensity as a function of thickness under several conditions. For reference, the solid line indicates $\mathrm{e}^{-\mu x}$ for tungsten at $4 \mathrm{MeV}$. The bremsstrahlung beam, shown in 
blue circles, initially drops off faster in intensity and then flattens out at around $4 \mathrm{~cm}$. Two different effects contribute to the nonlinear behavior: beam hardening, as lower energy portions of the spectrum are preferentially attenuated, and scatter, which contributes additional intensity. To distinguish these two effects, we also plot the transmitted intensity for the primary beam only, shown as red circles. The initial faster falloff persists, as the softer components of the spectrum are more heavily attenuated. It should be noted that for qualitative imaging, this effect can be beneficial: small, less attenuating features are more easily observed while still maintaining penetration for larger objects. However, this effect interferes with quantitative techniques, such as tomography. The nonlinearity in the primary-only results is much less than in the total, indicating that scatter plays a large role in the nonlinear behavior as the tungsten thickness increases.

For comparison, we also plot the transmitted intensity for the MPS, in green circles. The MPS is highly linear down to approximately $10 \mathrm{~cm}$ of tungsten, and then begins to flatten out, presumably due to scatter. The MPS does not suffer from beam hardening due to its much narrower spectrum, and because of the small beam divergence illuminating very little outside the object scatter effects are considerably reduced. Note that no collimator or cutoff is assumed on the MPS beam at large angles/lower energies. Such a collimator might further improve performance.

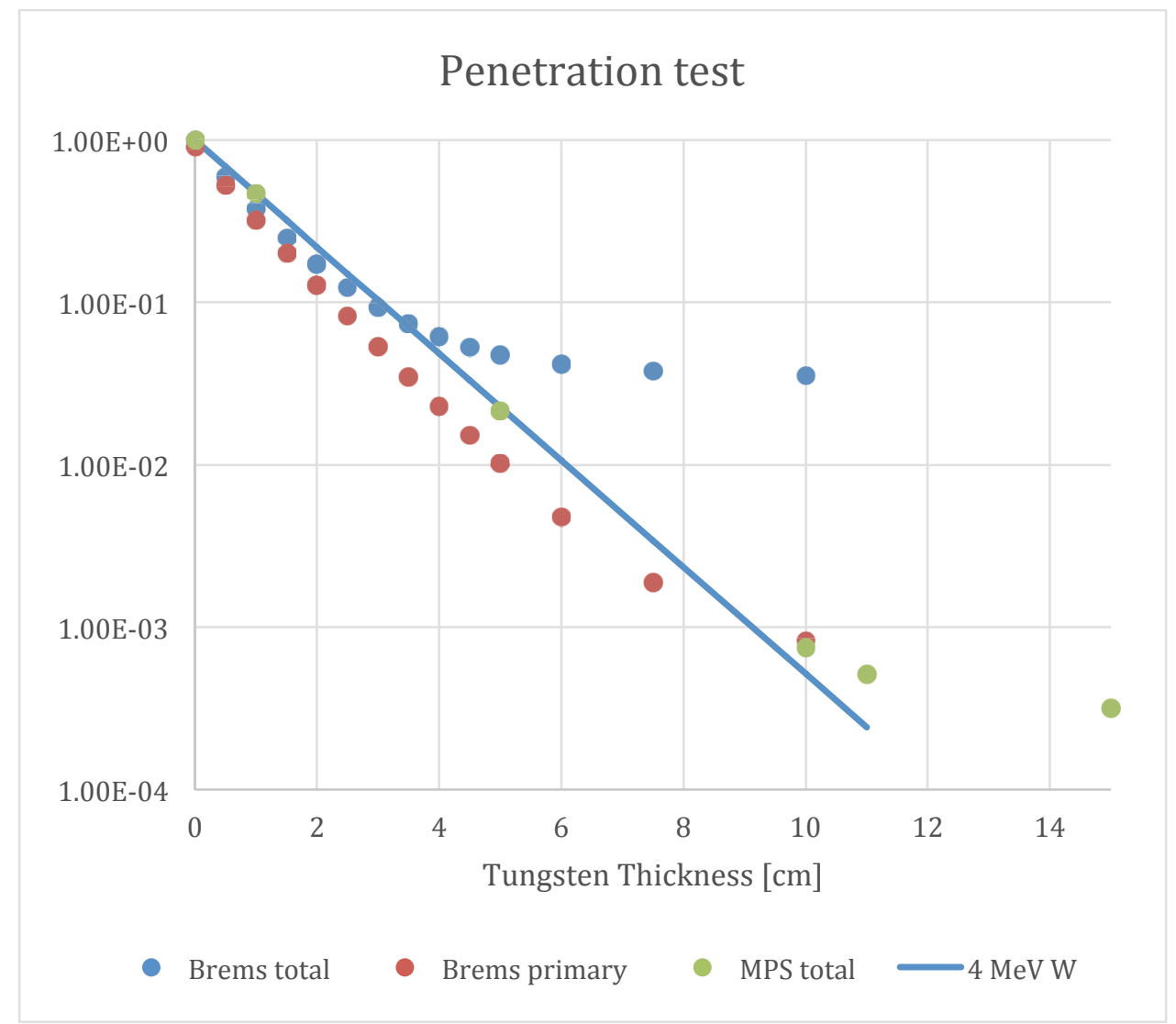

Figure 45. Transmitted intensity as a function of tungsten thickness in $\mathrm{cm}$. 


\section{II.4.6 Impact on Application and MPS Requirements}

We have seen that with the current geometry, the MPS would readily support micron-scale resolution. There is no indication that factors other than geometric spot size overwhelm the benefits of the small spot size, although very thin objects may suffer due to low contrast in the high-energy beam. A $6.5-\mathrm{m}$ standoff is not needed for the MPS from the viewpoint of spatial resolution.

Both beam hardening and scatter produce artifacts in tomography, which relies on an assumption of logarithmic attenuation. In the case of beam hardening, artifacts known as "cupping" are produced, where the attenuation in the middle of an object appears to be lower than the outer regions [108]. This can be corrected via dual-energy imaging, or with knowledge about the object material, but in general will interfere with high-quality quantitative tomography reconstructions.

Artifacts due to scatter are similar to beam hardening-inner and outer regions of an object appear to have different attenuation due to the relative contribution of scatter to the projections. Without correction, scatter-to-primary ratios (SPRs) as low as 0.05 to 0.10 can impair reconstruction [107]. This is a serious issue at high energies, and in fact the SPR for the linac system is around 0.10 even before an object is present. Various methods are possible to reduce or mitigate scatter, either by physically reducing it or by estimating its magnitude via modeling, but it is notable that the low-divergence beam geometry of the MPS is advantageous in reducing these artifacts.

The small spot size of the MPS might be most efficiently used for high-resolution imaging in a magnified geometry, where the object is near the source and the detector further away. A steered beam with low divergence may be used for a magnified geometry, although some oversampling may be necessary at the front of the object in order to ensure coverage at the back. A diverging beam could be beneficial for magnified imaging, at the expense of increased scatter and increased spectral bandwidth. This would require further study to evaluate.

While the resolution tests suggest that an MPS can achieve $1 \mu \mathrm{m}$ resolution, there are other constraints that will limit the spatial resolution. The resolution evaluation was done with an ideal detector, so detector resolution is one such constraint. This concern could be relaxed if a magnified geometry were used.

Another constraint is the transmitted flux delivered to the detector. Assuming the same number of photons per pixel is required for an MPS-based imaging system as the current CoLOSSIS, the improvement in resolution may be limited by the relative intensities of the two systems. For instance, the $2 \%$ energy width $200 \mathrm{kHz}$ MPS-based system, which could provide roughly 3 times the photons per unit area per unit of time as CoLOSSIS, would enable the reduction of the spatial resolution by roughly a factor of almost 2 compared to CoLOSSIS for equal exposure time. This estimate is conservative, as the reduced scatter and hardening effects may enable the resolution of even smaller features in equal time. Use of longer exposure time to increase flux delivered per pixel would allow the MPS-based source to reach its full potential resolution, giving the potential to image micron-scale features.

The initial calculations and modeling for imaging of nuclear warheads as part of stockpile stewardship program using MPS-based systems suggest that there could be significant improvements compared to the existing bremsstrahlung-based solution. These improvements could be leveraged to improve some combination of spatial resolution and measurement throughput, depending on what would be most beneficial. If the focus is on resolving smaller spatial features, an MPS-based system has the potential to reduce the size of observable features by a factor of 2 or more in equal exposure time, and potentially down to micron-scale resolution 
with longer exposure time compared to CoLOSSIS. The primary conclusion from this effort is that it would be worthwhile exploring the feasibility of this approach with higher fidelity modeling.

High resolution imaging for stockpile stewardship may provide a valuable tool for treaty and dismantlement verification involving nuclear warheads. Discussions with stakeholders in these communities identified a strong interest to "fingerprint" individual weapons by deriving an intrinsic unique identifier (I-UID). High-resolution imaging may provide that I-UID. For instance, the rotation of various screw/bolt heads may serve as the non-sensitive fingerprint to form an IUID. It would be necessary to demonstrate that imaging can be performed on non-sensitive components of a warhead without revealing any sensitive information. If an MPS-based system were already used in stockpile stewardship efforts, familiarity with the technique and experience using it on the stockpile would ease the transition for use in treaty verification. The driver for using an MPS-based system for this application is the higher flux, better imaging resolution, and lower imaging artifacts, which could enable shorter measurements, typically an hour at most, required by treaty verification.

For stockpile stewardship, installation in a facility of comparable scale to COLOSSIS is likely acceptable. Hence size is not a stringent constraint for that application. For treaty measurements, similar operational constraints apply to those outlined in the conclusion of section II.3. The example calculations reported here used a $4 \mathrm{MeV}$ MPS photon beam with a $2 \%$ energy spread, 2 $\mu \mathrm{m}$ beam emission spot, $0.42 \mathrm{mrad}$ divergence at $100 \mathrm{~cm}$ will cover an area of $0.56 \mathrm{~mm}^{2}$ delivering $10^{7}$ photons/shot, at $200 \mathrm{kHz}$ repetition rate. However, this energy spread is much narrower than is required for radiography (see also Section II.1), and correspondingly the number of photons per shot is conservative. Other pairs of yield and repetition rate could also be considered and could give similar performance: for example, $10^{8}$ photons/shot at $20 \mathrm{kHz}$. 


\section{II.5. Nuclear Safeguards Applications}

\section{II.5.1. Introduction}

Of principal interest in nuclear safeguards is the timely detection of the diversion of nuclear material, which relies heavily on material accountancy. Future MPS-based systems could address several accountancy challenges employing radiography and NRF techniques. Because the safeguards applications considered here vary in nature and different methodologies apply, MPSbased system capabilities and photon source requirements are discussed separately for each of the initially identified potential MPS applications in safeguards. These include:

- Spent fuel dry-storage cask content verification.

- Assay of spent fuel assemblies.

- Materials characterization in accident scenarios.

- Material accountancy and Pu monitoring in pyroprocessing.

- Verification of $\mathrm{UF}_{6}$ cylinder enrichment.

- Verification of fresh fuel in transport containers.

Dry storage cask content verification had been identified in the initial assessment [1] as a priority for further simulation studies because it is currently an active area of research and of interest in safeguards and an MPS-based system could provide new capabilities. Detailed transmission calculations and radiation transport simulations were conducted to establish feasibility of performing scans through casks of different designs that would reveal partial loading or missing assemblies. Simulations and results are described in Section II.5.2.

Other safeguards applications were considered briefly. Assay of spent fuel assemblies, materials characterization in accident scenarios, and material accountancy and Pu monitoring in pyroprocessing, are all addressed by transmission NRF enabled by a MPS producing an intense narrow energy-spread photon beam. Initial assessments described in Sections II.5.3, 5.4, and 5.5 indicate that the impact of a future MPS that meets the challenging requirements of these applications could be significant. However, none of these three applications were identified as high priority for international safeguards. They were thus identified as applications to consider at a later time, and no detailed simulation studies were performed. For enrichment verification of $\mathrm{UF}_{6}$ cylinders and verification of fresh fuel in transport, either no capability improvement could be identified or it seemed unlikely that MPSs could have an impact on these applications. Only initial calculations were conducted, which are described in sections II.5.6 and II.5.7.

\section{II.5.2. Spent Nuclear Fuel Dry Storage Cask Content Verification}

\section{II.5.2.1. Background and Current Status}

Dry cask storage has proven to be effective for safely storing spent nuclear fuel (SNF) for relatively long periods of time until a permanent solution is available, and many countries have adopted dry storage systems as an option to store their SNF. Whereas the CANDU dry storage system has re-verification tubes in its design, none of the dry storage cask systems for light-water reactor (LWR) spent fuel have re-verification features. The "fingerprinting" technique has been considered to re-establish the continuity-of-knowledge (CoK) in the event of loss of CoK on the safeguarded fuel, but the effectiveness of the fingerprinting method is questionable, as the radiation signatures from the innermost assemblies do not reach outside of the storage surfaces. The IAEA is actively pursuing a method that allows re-verification of LWR spent fuel stored in dry casks without the need for a reference baseline measurement or fingerprinting [109]. We 
understand "re-verification" to mean the verification that all SNF assemblies are present.

This is a challenging problem because dry storage casks are large cylinders that can hold 24 or more pressurized water reactor (PWR) assemblies or 68 boiling water reactor (BWR) assemblies. The casks' walls and lids are made of thick steel and additional neutron shielding or of relatively thin steel and a thick concrete overpack. Two examples of dry storage casks are shown in Figure 46. These are the TN-68 cask (capacity: 68 BWR assemblies) [110] and the Holtec International HI-STORM 100 (capacity: 24 PWR assemblies. Another example of a dry storage cask is the MC-10 cask (capacity: 24 PWR assemblies, overall length: $495 \mathrm{~cm}$, overall diameter: $274 \mathrm{~cm}$, lid thickness: $\sim 34 \mathrm{~cm}$, wall thickness: $\sim 25 \mathrm{~cm}$, bottom thickness: $\sim 25 \mathrm{~cm}$ ) that is used in some of the analysis reported here.

At present, the canisters are mostly stored upright in rows with several meters in between casks, which would provide room for larger equipment. In other storage facilities (for example, Susquehanna Dry Cask Storage) dry casks are stored horizontally in above-ground concrete bunkers and would likely need to be pulled out to gain access for measurements. These casks would need to be pulled out to gain access for measurements. Re-verification is expected to be infrequent, so rather long measurement times-hours or possibly longer-may be acceptable.
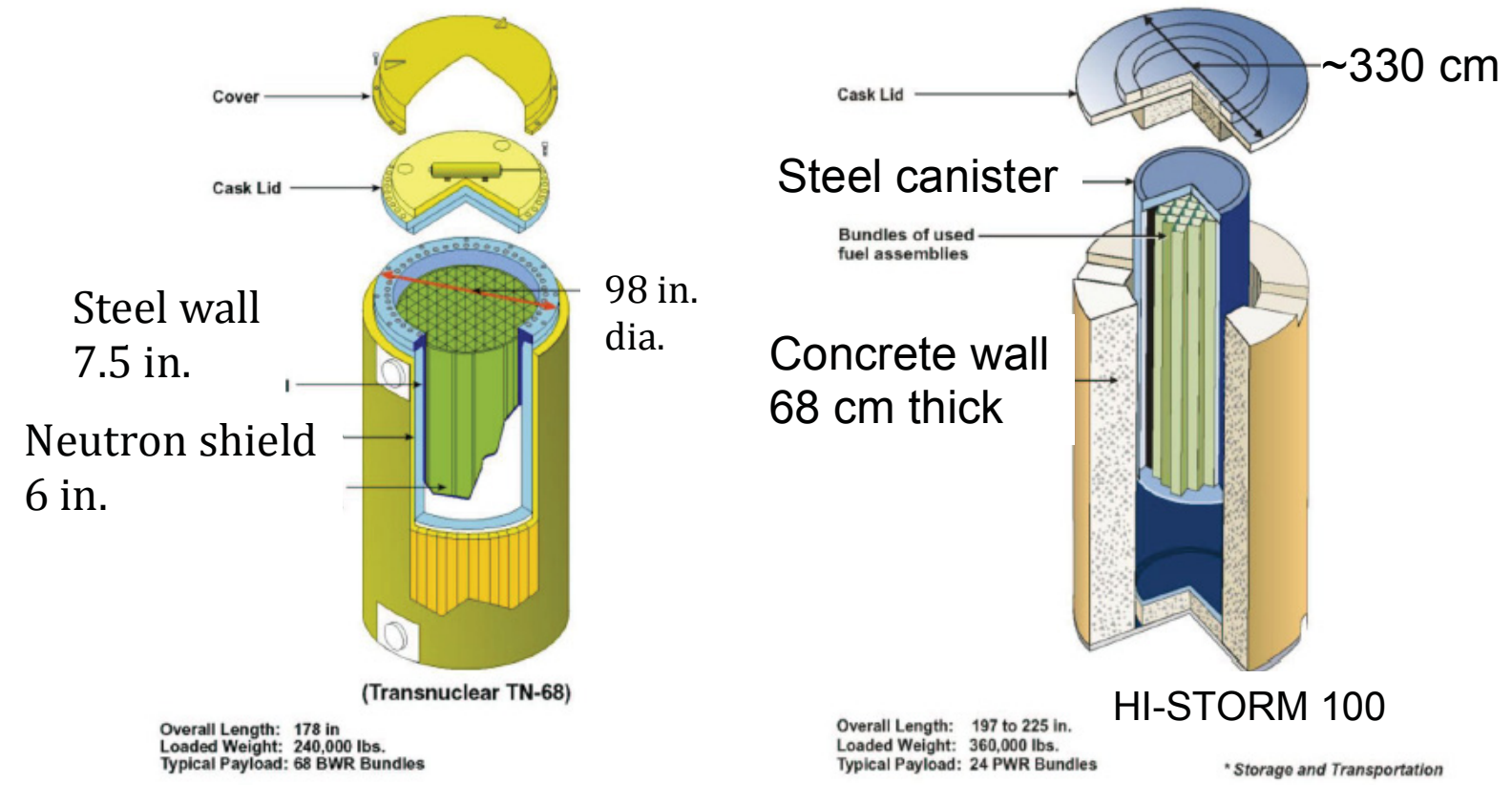

Figure 46. Two dry storage cask types. On the left is a Transnuclear TN 68 cask with a thick steel wall and a neutron shield; on the right is a HI-STORM 100 cask consisting of a steel cylinder in a concrete overpack.

Currently, methods are under development to enable re-verification. A Compton Dry-Cask Imaging Scanner (CDCIS) was developed at INL that scans the gamma emission across the top of the cask [111]. The system's ability to detect empty vs. full storage positions in the cask was demonstrated on a Westinghouse MC-10 cask. However, the scanner was not able to distinguish empty vs. full positions for casks in Doel (Belgium), which was likely due to the presence of a thick steel shield over the lid of the cask that introduced too much scattering. [111] A cosmic ray muon imaging system is under development and has been tested at INL's dry cask test bed on a MC-10 cask. [112]. The preliminary results indicate that missing fuel bundles can be detected after 100 hours of exposure. The report states that the detection system could be improved for 
better performance. An MPS-based technique was evaluated to determine potential utility, and comparison between methods should continue as each technique develops further.

\section{II.5.2.2. Potential Capabilities and Advantages of MPS-based System}

The thick walls of dry storage casks and the additional attenuation and scatter in the stored assemblies make radiographic measurements for the verification of the canister content very challenging. Conventional radiography with a broad-beam bremsstrahlung source cannot be used because the very large scatter contribution makes it impossible to achieve sufficient contrast. The scatter contribution can be greatly reduced or eliminated, and thus the contrast drastically improved, in transmission radiography with a small-diameter ("pencil") photon beam. This offers the possibility to scan the cask with a high-flux pencil beam and measure the transmitted photon flux. Along a line through an unoccupied storage position the transmission would be significantly increased and the absence of an assembly would be detected.

Scanning a spent fuel cask by measuring the transmission lengthwise through the cask, i.e., with the beam parallel to the fuel rods, is the simplest approach for determining if an assembly position is filled or empty. Transmission through an empty slot would be significantly higher than through an assembly as the fuel rods absorb and scatter the beam. With a fill factor on the order of $50 \%$ for an intact fuel assembly, the transmitted photon flux averaged over the assembly cross section would be two times higher for the empty slot. With a beam diameter close to that of a fuel pin, i.e., $1 \mathrm{~cm}$ or smaller, individual pins could be resolved with very high contrast, and scanning across a target position could not only reveal the presence or absence of an assembly but also detect missing pins.

A lengthwise transmission measurement is a good match for horizontal casks, but for upright casks the detector would need to be placed underneath and the MPS moved across the top for performing transmission measurements. Because the casks can weigh 100 tons and are hard to move, a lengthwise measurement on upright casks may not be practical, and transverse measurements have to be considered. The presence of fuel bundles in the cask can in principle be verified by a limited number of transmission measurements that probe all assembly positions. Because the pins in an assembly are arranged on a lattice and the assemblies in the cask are typically lined up in rows and columns, with narrow gaps in between, a more detailed assessment of transverse scanning is required to find best scanning techniques and determine capabilities.

Bremsstrahlung beams can be tightly collimated but the photon flux generated with available electron linacs is insufficient for penetrating a cask filled with fuel bundles. A high power $(9 \mathrm{MeV}$, $10 \mathrm{~mA}$ ) Rhodotron accelerator could produce a time-averaged bremsstrahlung photon flux in a few mrad divergence beam that is comparable to the flux of a Thomson source, but discrimination against the radioactive background would likely not be possible. The typical cw operation of a Rhodotron would preclude detector gating such as could be used with a Thomson source and the broad energy spectrum with the highest photon flux at background energies would make energy discrimination very challenging. In addition, a Rhodotron is a large and heavy accelerator that would make transport to and positioning next to a dry-storage cask impractical.

A Thomson MPS offers several advantages that could enable transmission measurements for detecting and locating empty vs. full assembly storage positions in a dry storage cask. Most importantly, such sources produce low divergence photon beams and are expected to deliver a high flux on target, suitable cm-scale photon beam spot sizes, and high repetition rates. Our preliminary analysis [1] indicated that photon transmission measurements with high intensity beams of 6-8 MeV photons and with a small divergence of several mrad could verify the content of a spent fuel dry-storage cask. Based on analytical calculations unscattered photon transmission 
probabilities lengthwise through a cask of $10^{-7}$ to $10^{-10}$ were estimated for a range of lid and bottom thicknesses. This indicates that longitudinal measurements could be performed with beam intensities on the order of $1 \times 10^{11} \mathrm{ph} / \mathrm{s}$. These general parameters were used to set up detailed simulations that included realistic models of fuel casks and backgrounds, and of photon beam propagation.

The simulation study section below presents higher-fidelity calculations and modeling results to evaluate "re-verification", i.e., to verify that all SNF assemblies are present. The section begins with a high-level summary of the decision criteria and observable signals. Subsections quantify capability for various cask orientations, types and scan possibilities.

\section{II.5.2.3. Assessment of MPS Performance: Simulations}

Detailed transmission calculations and radiation transport simulations were conducted to establish feasibility of performing scans through casks of different designs that would reveal partial loading or a missing assembly. Backgrounds, which constrain detection performance, were first analyzed. Calculations were then conducted for transverse and longitudinal scanning. The simulations were used to identify suitable transmission measurement techniques, determine contrast and penetration, and understand how best to quantitatively determine verification capabilities across a range of different cask designs. This includes the possibility that the regular arrangement of assemblies and pins in the cask can be exploited to determine the assembly basket occupancy more efficiently. The results evaluate the ability to show that the absence or presence of an assembly can be detected and determine performance parameters. The calculations were more extensive for analyzing the transverse transmission approach because of the strong beam attenuation by the assemblies and the inhomogeneity and structure of the spent fuel assemblies. In order to more accurately estimate transmission probabilities for various beam positions through cask and assemblies and to include scattering effects, detailed MCNP simulations were performed.

\section{Simulation Setup: Estimate of Spent Fuel Gamma-ray Background}

The spent nuclear fuel gamma-ray background seen on the outside of the cask by the transmission detector could impact measurement sensitivity and photon flux requirements. The background was estimated using the codes ORIGEN [113] for calculating fuel depletion and MCNP [114] for modeling the radiation transport. The simulation was done for PWR fuel bundles from the Diablo Canyon Power Plant with a cooling time of 3 years [115]. Because 3 years is the earliest that spent fuel can be placed in a dry storage cask, this is the worst-case background intensity. In practice, the loss of continuity of knowledge and need for re-verification is more likely to occur for older casks that have stored fuel for many years; due to the longer spent fuel cooling times, the background for such casks is greatly reduced.

For the background simulation the cask was assumed to be filled with a homogenized mixture of 24 assemblies. Photons with energies from $0.25 \mathrm{MeV}$ to $3.5 \mathrm{MeV}$ (the highest energy photons emitted from spent fuel) were transported through the cask and the flux on the $5 \mathrm{~cm} \times 5 \mathrm{~cm}$ face of the detector, located at the $22.86 \mathrm{~cm}$ steel bottom of the MC-10 cask, was calculated. Figure 47 shows the simulated energy spectrum at the detector, and the configuration is shown in Figure 48. The energy-integrated background rate is $\sim 2,400$ counts per second. The pulse structure of a Thomson source, with its sub-picosecond duration beam pulses at repetition rates in the $\mathrm{kHz}$ range, enables rejection of the passive spent fuel background by gating the photon transmission detector with the beam, i.e., by rejecting counts between beam pulses. Assuming a gate width of 4 ns, conservative for a fast scintillation detector, and a $10 \mathrm{kHz}$ pulse repetition rate, the gated background rate is $\sim 0.1$ counts per second. Because this rate is well below the lowest 
transmission signal one would expect for either longitudinal or transverse scans (see estimates below), the radioactive background from the spent fuel is not expected to impede transmission measurements.

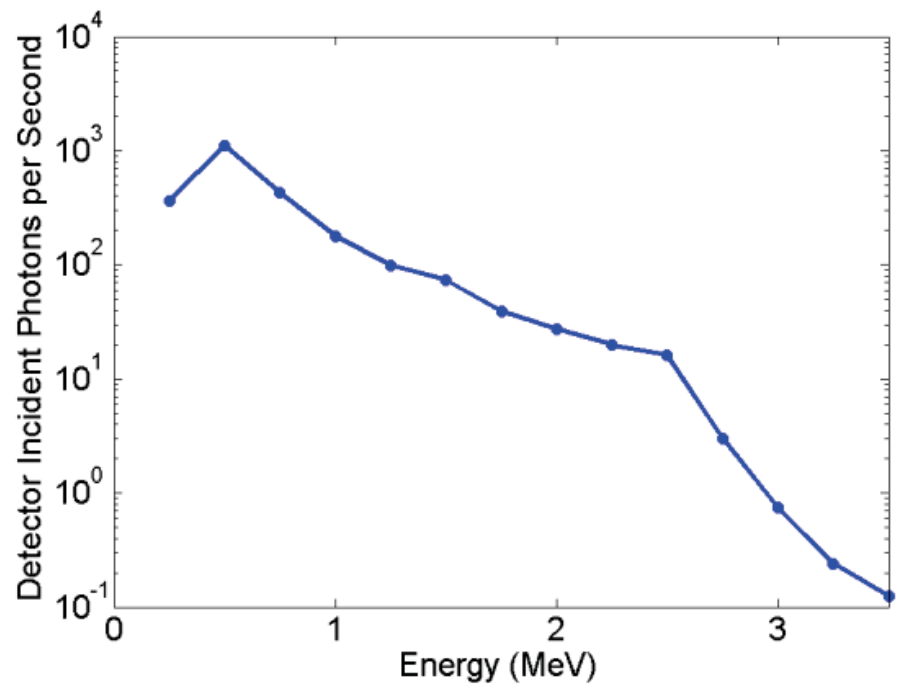

Figure 47. Simulated spent fuel background spectrum outside cask.
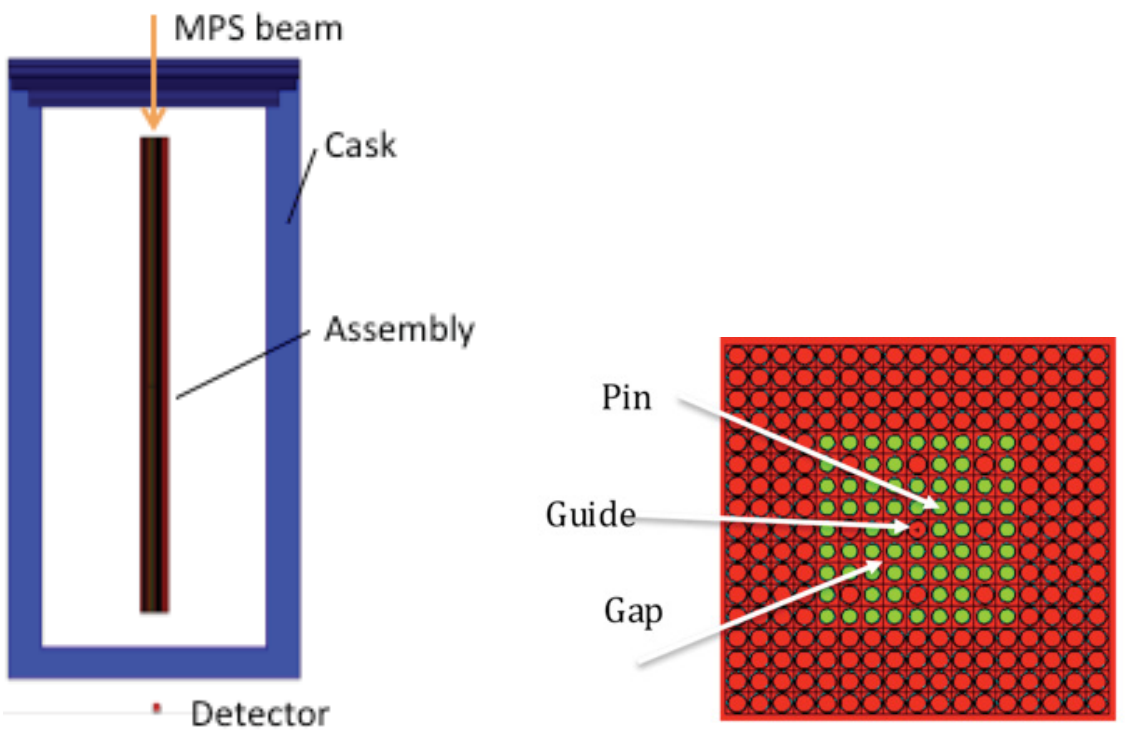

Figure 48. Geometry for MCNP simulation of the photon beam transport through cask with steel lid and bottom (MC-10). The beams enter from the top and are positioned on the assembly as shown on the right. The green dots indicate the pins included in the simulation. The $5 \mathrm{~cm}$ cube detector is positioned underneath the cask.

In addition to detector gating, an energy threshold could be applied at low transmission rates for which single photon counting is possible. An energy threshold of $3 \mathrm{MeV}$ would eliminate the background since almost all gammas from the radioactive decay of the spent fuel have energies below that threshold, as seen in Figure 47. However, operating in single photon counting mode 
and applying an energy threshold does not seem necessary. Cask background does not appear to be a significant restriction on measurements for pulsed, narrow emission angle MPSs such as Thomson sources.

\section{Results: Longitudinal Transmission Scan}

MCNP simulations were performed to evaluate longitudinal cask measurements. The simulations take into account beam divergence and assembly geometry, and include photon scattering. In a lengthwise transmission measurement the beam needs to penetrate the lid and bottom of the cask and the beam attenuation depends on their combined thickness. The lid thickness varies significantly across different cask types, and with the combined lid and bottom, thickness ranges from $\sim 60 \mathrm{~cm}$ to $\sim 100 \mathrm{~cm}$ of steel. Un-scattered photon transmission probabilities for an empty storage position range accordingly from $\sim 1 \times 10^{-6}$ to $1 \times 10^{-10}$. Transmission values for casks with a concrete overpack also fall within this range. Calculations established that the transmission is maximized for $\sim 8 \mathrm{MeV}$ photons. The simulations were performed for a simplified MC-10 cask and a $17 \times 17$ PWR assembly, but results can be extrapolated to casks with different lids and bottom plates by simply taking the change in attenuation into account.

An $8 \mathrm{MeV}, 30 \%$ FWHM photon beam with a spatial divergence of $3.5 \mathrm{mrad}$, representative of candidate Thomson photon sources (tuned for high photon yield instead of narrow energy spread), was transported through the simplified MC-10 cask [116] which had a combined thickness of $\sim 60 \mathrm{~cm}$ of steel in the lid and base attenuating the beam. The transmission was simulated for a beam through an empty slot (no assembly) and through a 17×17 PWR assembly [117] with representative pins [116]. The transmission through the assembly was calculated for three different beam locations corresponding to the beam being centered in the gap between pins, in a guide tube, and on a pin as indicated in Figure 48. For the chosen divergence of $3.5 \mathrm{mrad}$, the beam diameter of $\sim 2 \mathrm{~cm}$ at the far end of the assembly is smaller than the assembly width but larger than a single pin. In the simulated geometry that was shown in Figure 48, the transmitted photons are detected by a $5 \mathrm{~cm}$ cube detector that is positioned in line with the beam and underneath the cask. The number of photons on the front surface of the detector was tallied. The detector response was not simulated.

Figure 49 shows the energy spectra of the photons reaching the detector for the four simulated cases. The spectra are dominated by the broad peak around the beam energy, i.e., by the unscattered photons. The spectrum for the beam centered on a pin is not visible in this figure because the transmission probability is three orders of magnitude less than in the other cases. The probabilities for a photon being transmitted are listed in Table 27. Without an assembly in the beam's path, the transmission probability is simply determined by the thickness of lid and bottom. With an assembly in place and the beam centered on a gap or a guide tube the transmission is reduced by about a factor of two. If the beam is centered on a pin, the transmission is more than $1000 \times$ lower because the beam photons cannot transmit lengthwise through the pin. For beams centered in between these positions the transmission probabilities are in between the extreme values. Because the transmission differs by at least a factor of two, empty assembly slots can easily be identified. If the beam were scanned across an assembly in sufficiently small steps, a single missing pin could be detected. 


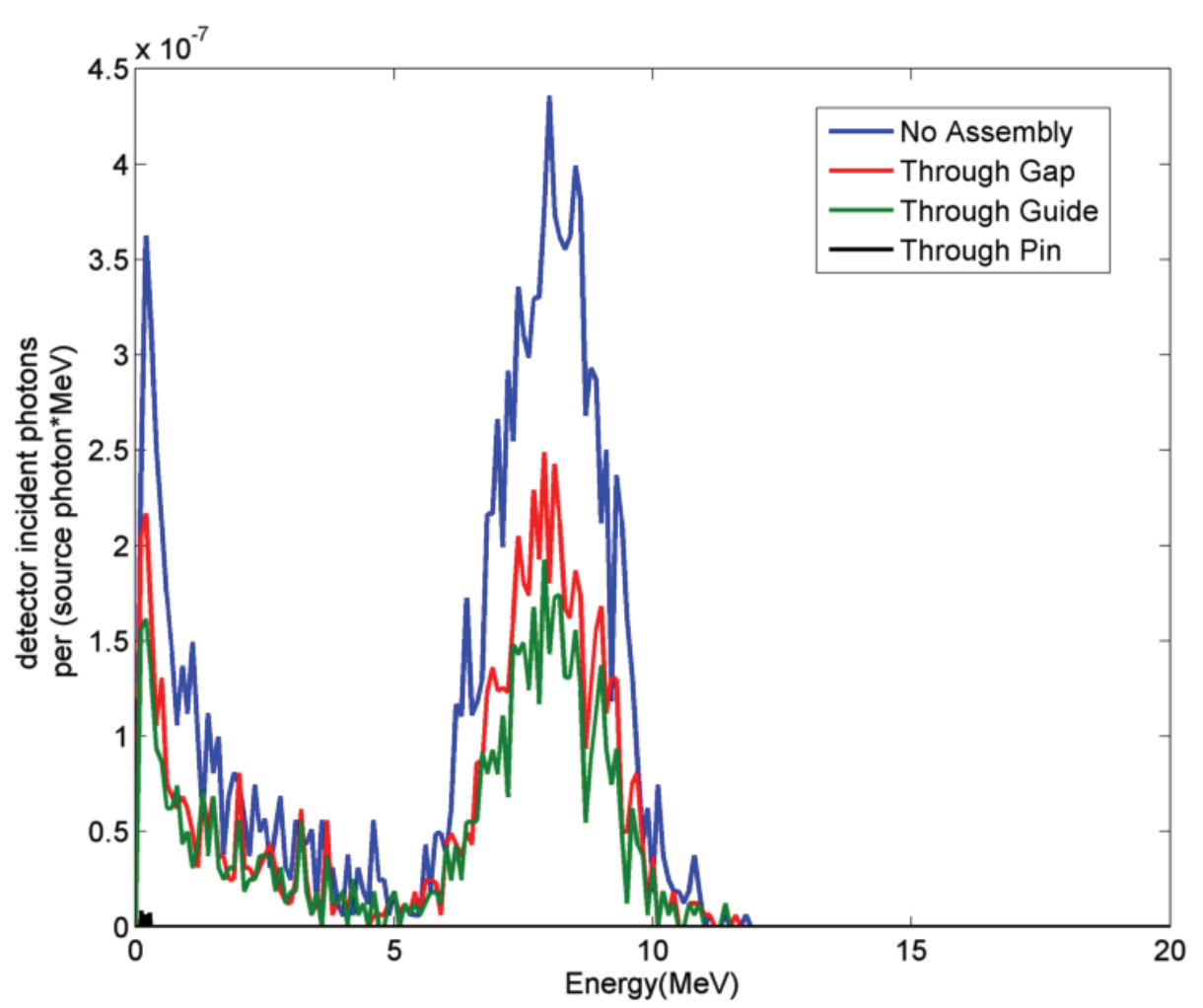

Figure 49. Transmitted photon spectra for the four simulated cases. The dashed black line, which represents the photon spectra for the beam centered on a pin, is not distinguishable from the $\mathrm{x}$ axis because the transmission probability is three orders of magnitude less than in the other cases.

Table 27: Number of photons per source photon entering the detector for longitudinal measurements through an MC-10 cask.

\begin{tabular}{|c|c|c|c|}
\hline No assembly & Through gap & Through guide & Through pin \\
\hline $1.4 \times 10^{-6}$ & $7.6 \times 10^{-7}$ & $5.7 \times 10^{-7}$ & $4.5 \times 10^{-10}$ \\
\hline
\end{tabular}

\section{Results: Transverse Transmission Scan}

While probing the assembly occupancy of a cask with longitudinal beams is the simplest approach, it may not always be practical. Most casks are standing upright, making it difficult to place source and detector above or underneath the cask. Moving the cask to an orientation where longitudinal scanning can be accomplished may be undesirable. In these cases scanning with a transverse beam would be necessary. Various methods for accomplishing this with a nearmonoenergetic, narrow-divergence beam were modeled using analytic calculations. The attenuation along lines through cask and assemblies was calculated based on mass attenuation coefficients [118] for the given geometry and materials. These analytical calculations did not include scattering or the effects of beam divergence. The analytical results were then validated against MCNP simulations, which do include scattering, for representative cases. This allowed analysis of a broad range of cases, while mitigating the required computation time. 
Various scanning configurations and methods were investigated using analytic calculations for a simplified MC-10 all-steel cask and for HI-STORM casks (Figure 46) that have a concrete overpack. Figure 50 shows the cross section of the MC-10 cask with a steel wall of $25.4 \mathrm{~cm}$ and loaded with twenty-four $17 \times 17$ PWR assemblies. The labeled assemblies were removed individually for different scans, so that discrepancies in the transmission map could be observed. Calculated was the attenuation through the cask for 55 beam chords to build up a transmission profile. The beams were scanned across the cask in several different patterns. Either the cask was scanned with parallel beams at a fixed angle in respect to the assemblies or with beams that originated from one spot (fixed source location) and traversed the cask at changing angles. Note that the later is more straightforward for MPS operations. Two examples are illustrated in Figure 51 , corresponding to source translation or rotation. The calculated transmission probability profiles are shown in Figure 52.

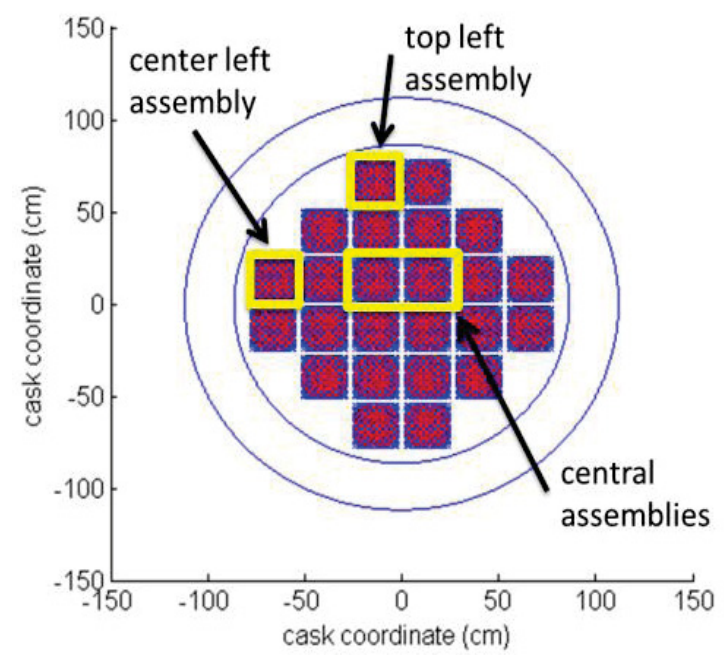

Figure 50. Simplified MC-10 steel cask model (blue outline) with 24 PWR assemblies, each $17 \times 17$ pins. The labeled assemblies (outlined in yellow) were removed individually to determine differences in attenuation for a missing assembly.
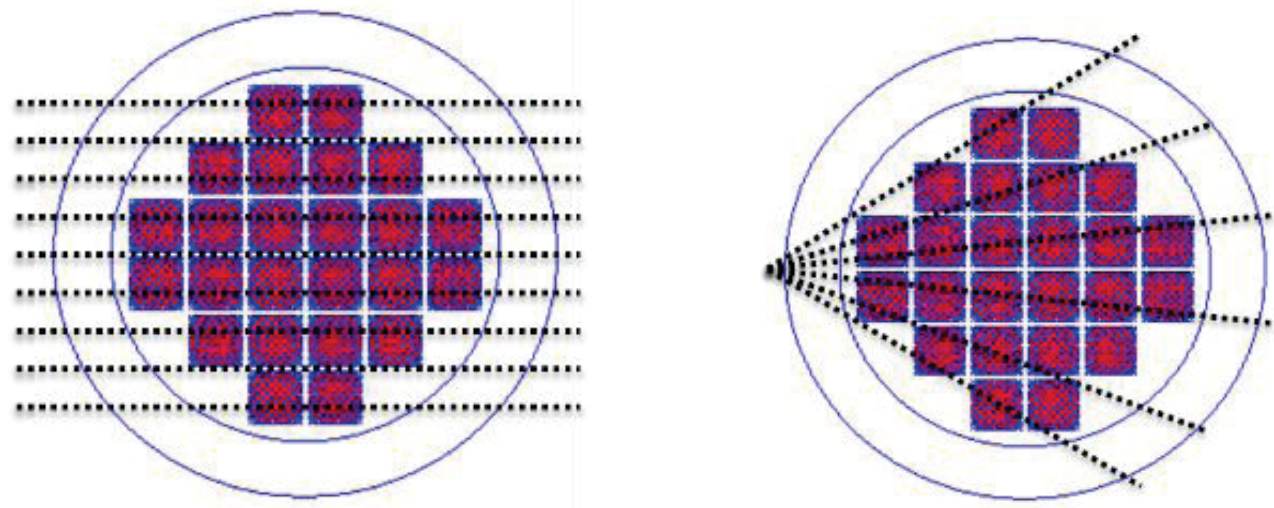

Figure 51. "Parallel" scan (left) and "fan" scan (right); the number of illustrated beams is reduced for clarity. 

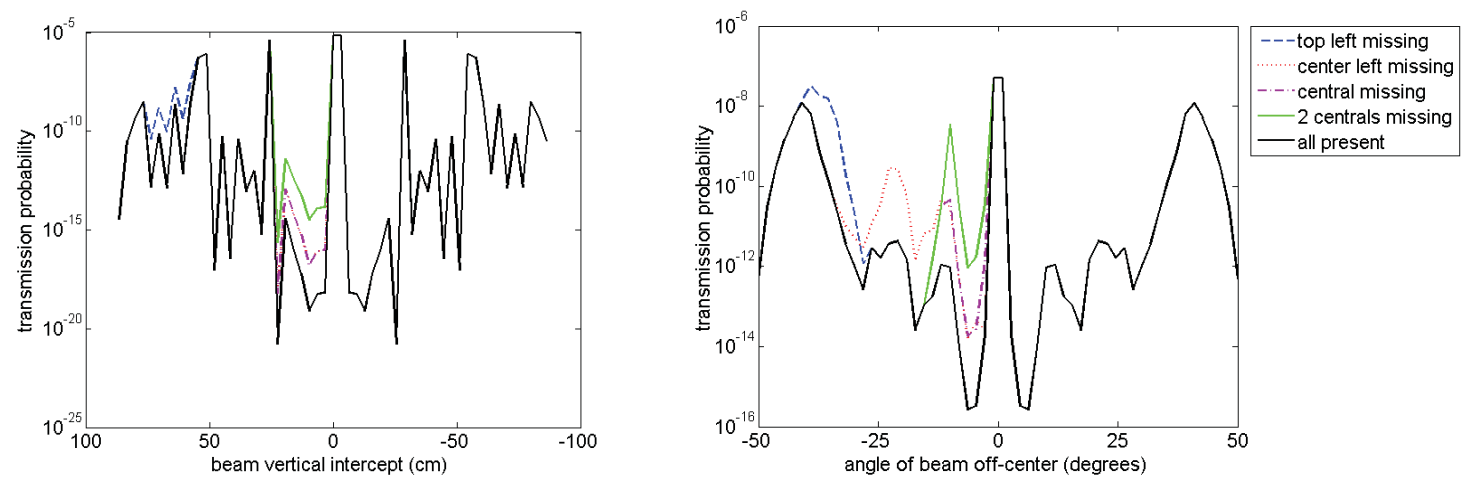

Figure 52. "Parallel" (left) and "Fan" (right) scan transmission probability maps. The black lines show the transmission probabilities with all assemblies in place. The colored lines give the transmission values with specific assemblies removed as indicated in the legend.

For the "parallel" scan, the transmission varies over many orders of magnitude, depending on how the beam lines up with the rows of pins in the assemblies. With removal of an assembly, the transmission increases roughly an order of magnitude but stays well below $1 \times 10^{-12}$ (which is considered the lower limit for transmission measurement, as explained below) for the central part of the cask. A rotated parallel scan for which the beams do not align with the rows of assemblies and pins gives better results, but for certain configurations the transmission through the center of a cask can still be very low. Generally, better results are achieved with a "fan" scan such as illustrated in Figure 52 right. Over a range of source positions and "fan" beam orientations, alignment of the beam with rows of fuel pins can be avoided, and the transmission through cask can hence be kept high enough that assemblies can be measured.

In order to determine source positions and fan beam orientations that give the best results, calculations were performed for "fan" scan orientations in 5 degree increments up to a 45 degrees as illustrated in Figure 53. Transmission probabilities were calculated for the fully loaded cask and for cases with some assemblies removed as indicated in Figure 54. Figures 55 through 58 show the transmission profiles for several source orientations. For source positions between 225 and 205 degrees the transmission probability increases by an order of magnitude in the case of a missing assembly to above the measurable probability of $10^{-12}$ across the cask. This indicates that the measurement would be sensitive to a missing single assembly in any location. 



Figure 53. Analyzed source orientations for "fan" scans of an MC-10 cask.

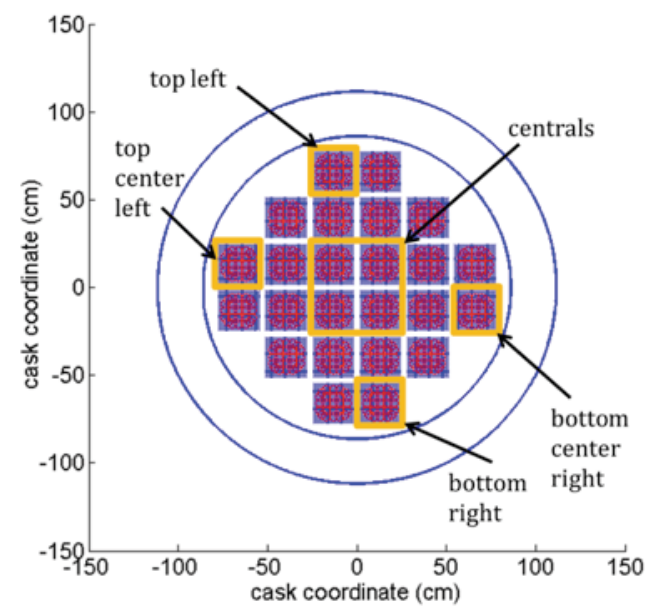

Figure 54. Assemblies marked in yellow were removed as indicated in the transmission profile graphs below. 


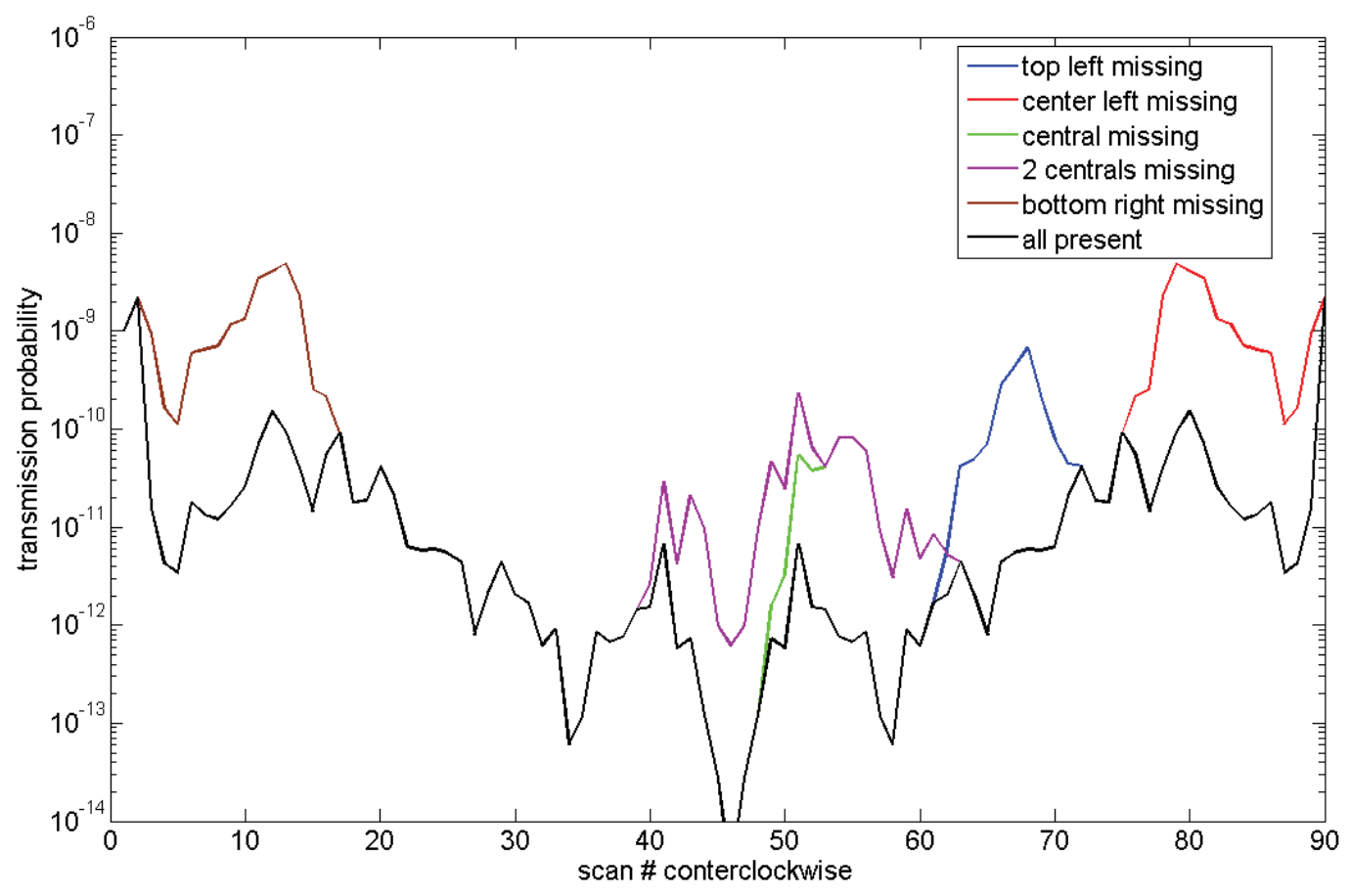

Figure 55. Transmission profiles for "fan" scans of a MC-10 cask with the source at $225^{\circ}$. The black lines show the transmission probabilities with all assemblies in place. The colored lines give the transmission values with specific assemblies removed as indicated in the legend.

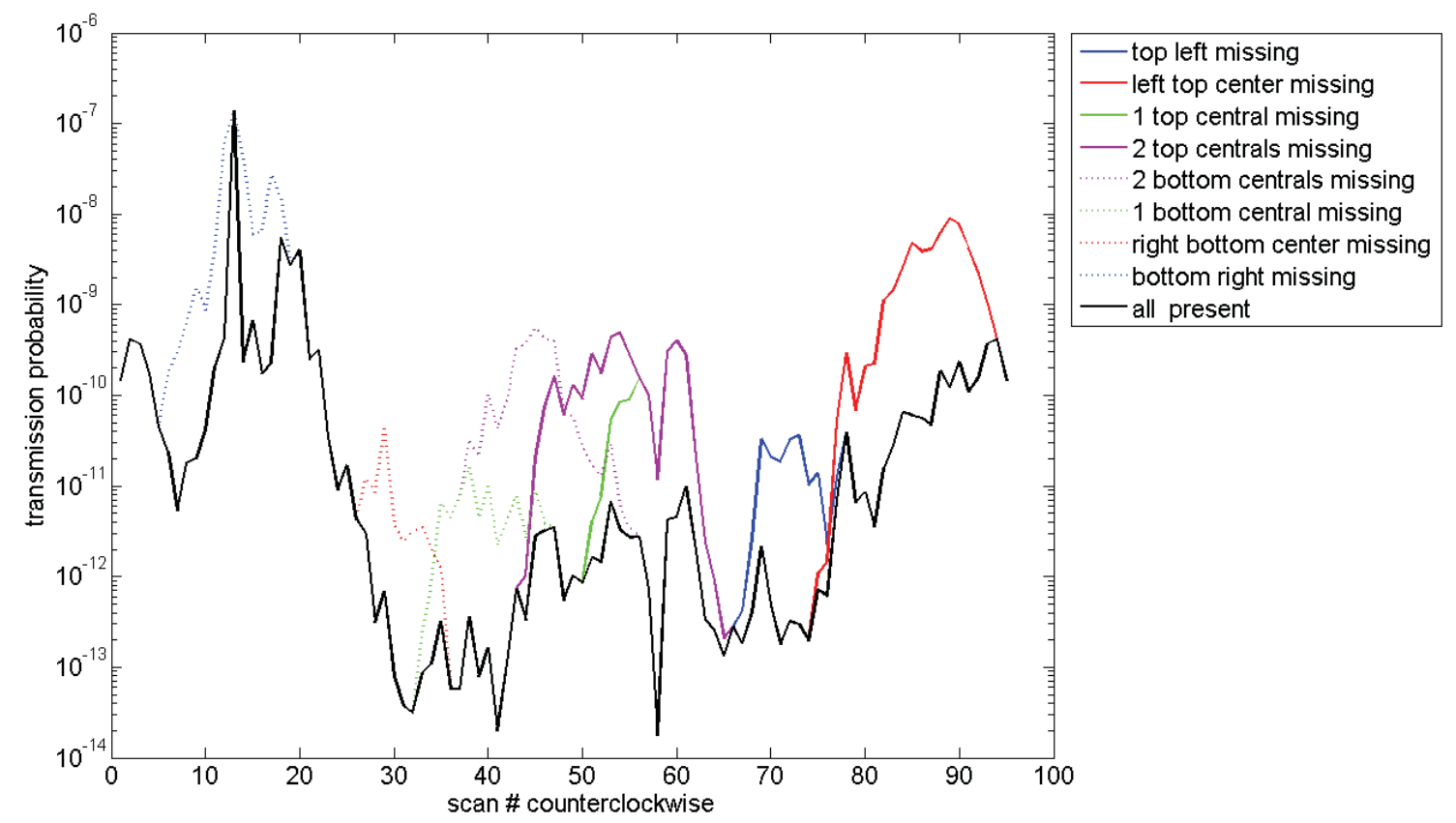

Figure 56. Transmission profiles for "fan" scans of a MC-10 cask with the source at $215^{\circ}$. The black lines show the transmission probabilities with all assemblies in place. The colored lines give the transmission values with specific assemblies removed as indicated in the legend. 


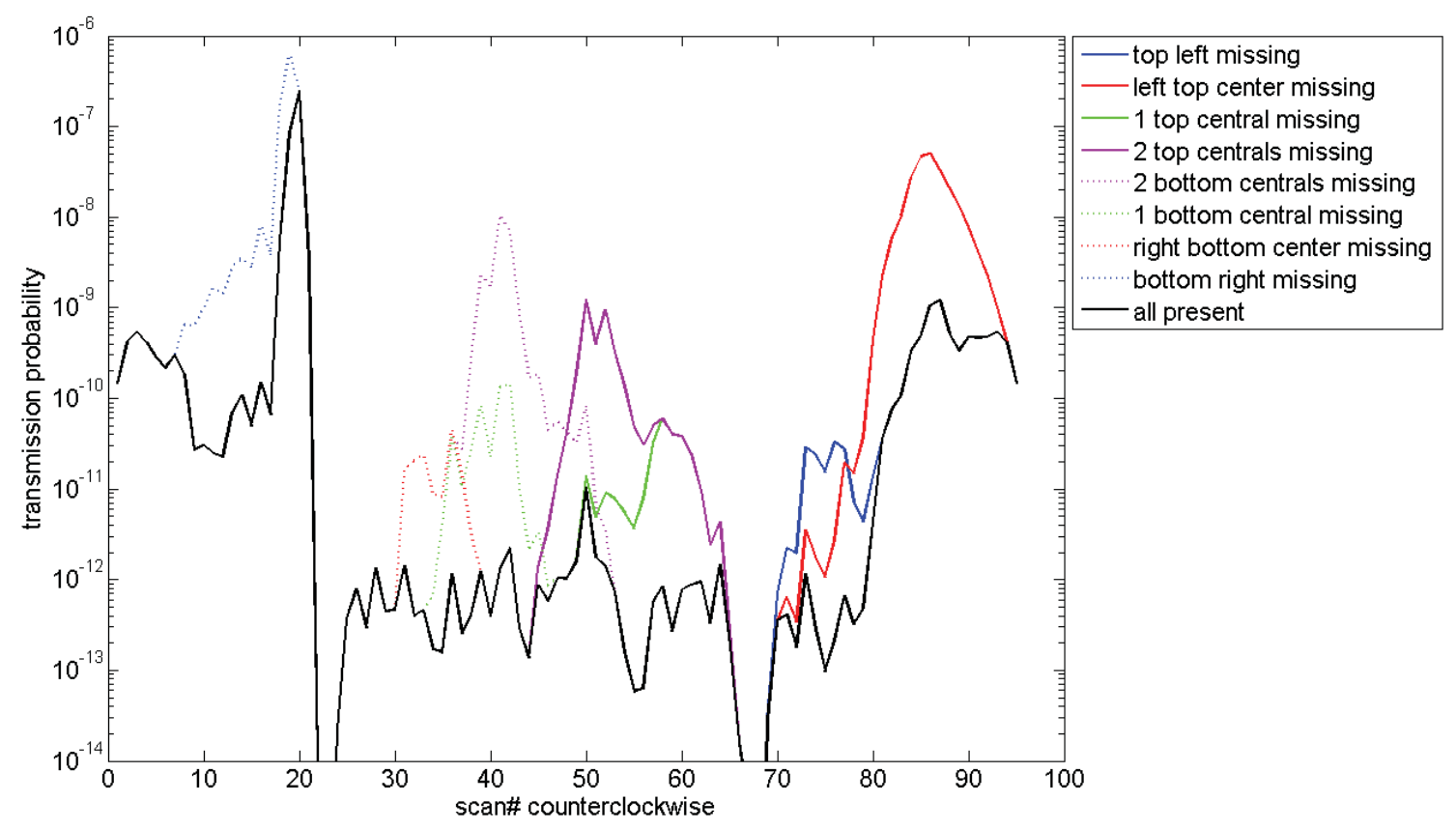

Figure 57. Transmission profiles for "fan" scans of a MC-10 cask with the source at $205^{\circ}$. The black lines show the transmission probabilities with all assemblies in place. The colored lines give the transmission values with specific assemblies removed as indicated in the legend.

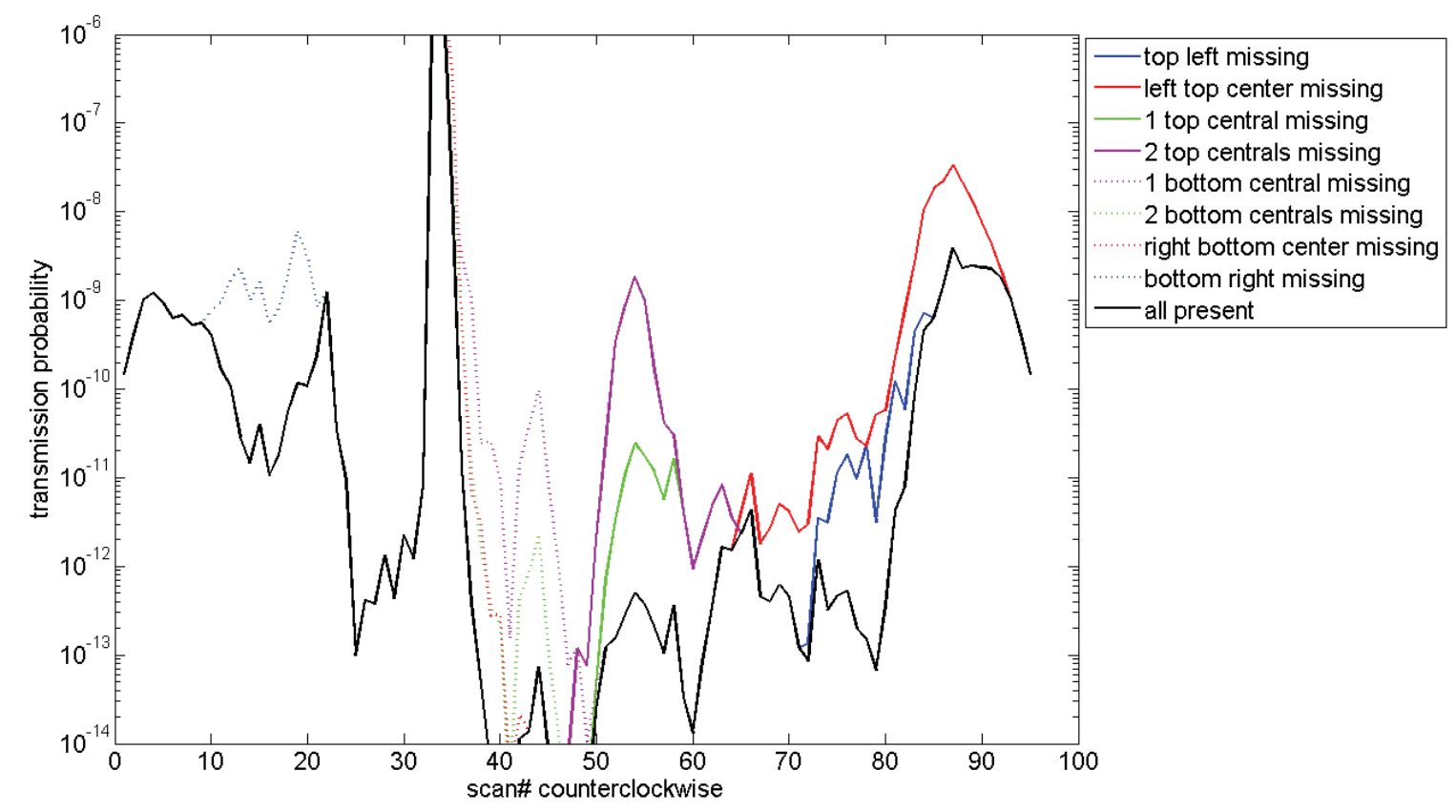

Figure 58. Transmission profiles for a "fan" scans of a MC-10 cask with the source at $195^{\circ}$. The black lines show the transmission probabilities with all assemblies in place. The colored lines give the transmission values with specific assemblies removed as indicated in the legend. 
In addition to the analysis of transverse scans of a MC-10 cask, calculations were performed for a model HI-STORM 100 cask with two different loadings of 32 PWR assemblies and 68 BWR assemblies as shown in Figure 59. This is a different type of cask in which the assemblies are stored in a steel cylinder with relatively thin walls that is then placed into a concrete overpack. The transmission profiles for 205 degree "fan" scans (Figures 60 and 61) indicate, similarly to the transmission profiles for the MC-10 cask, that the transmission probabilities increase by more than an order of magnitude for the MPC-32 loading and by a factor of a few for the MPC-68 loading if an assembly is missing. In both cases the transmission probability is higher than 10-12 when an assembly is missing, and measurements could be performed in acceptable times as discussed below. As in the case of the MC-10 cask, the measurement would be sensitive to a missing single assembly in any location.
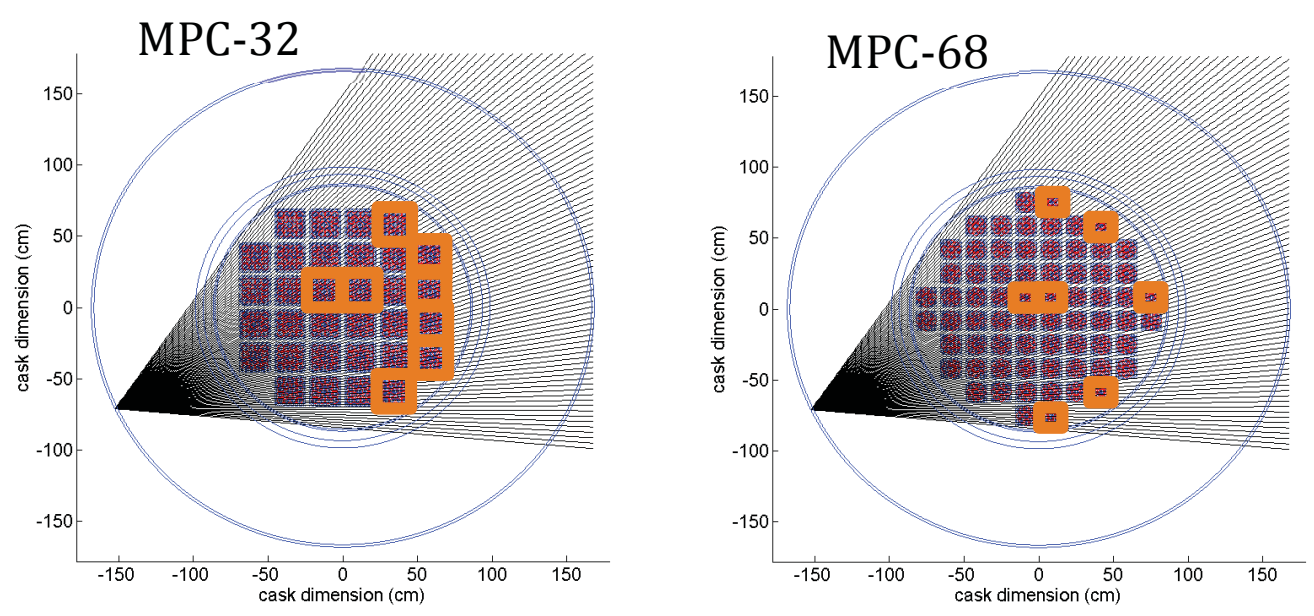

Figure 59. Geometry of transmission profile calculations for HI-STORM MCP-32 (left) and HI-STORM MCP-68 (right) casks with the source for the "fan" scan at $205^{\circ}$. 


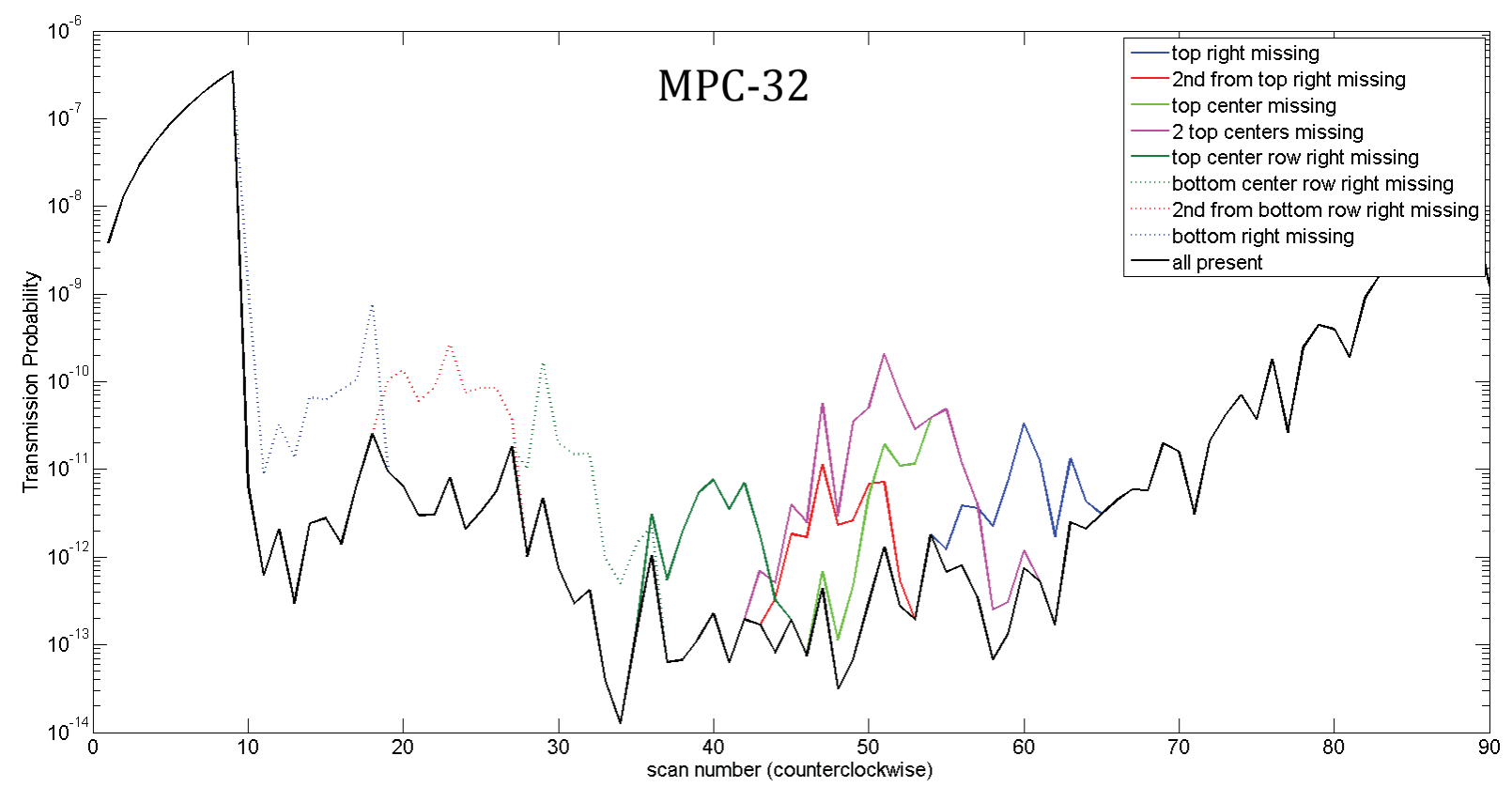

Figure 60. Transmission profiles for $205^{\circ}$ "fan" scans of a HI-STORM MPC-32 cask. The black lines show the transmission probabilities with all assemblies in place. The colored lines give the transmission values with specific assemblies removed as indicated in the legend.

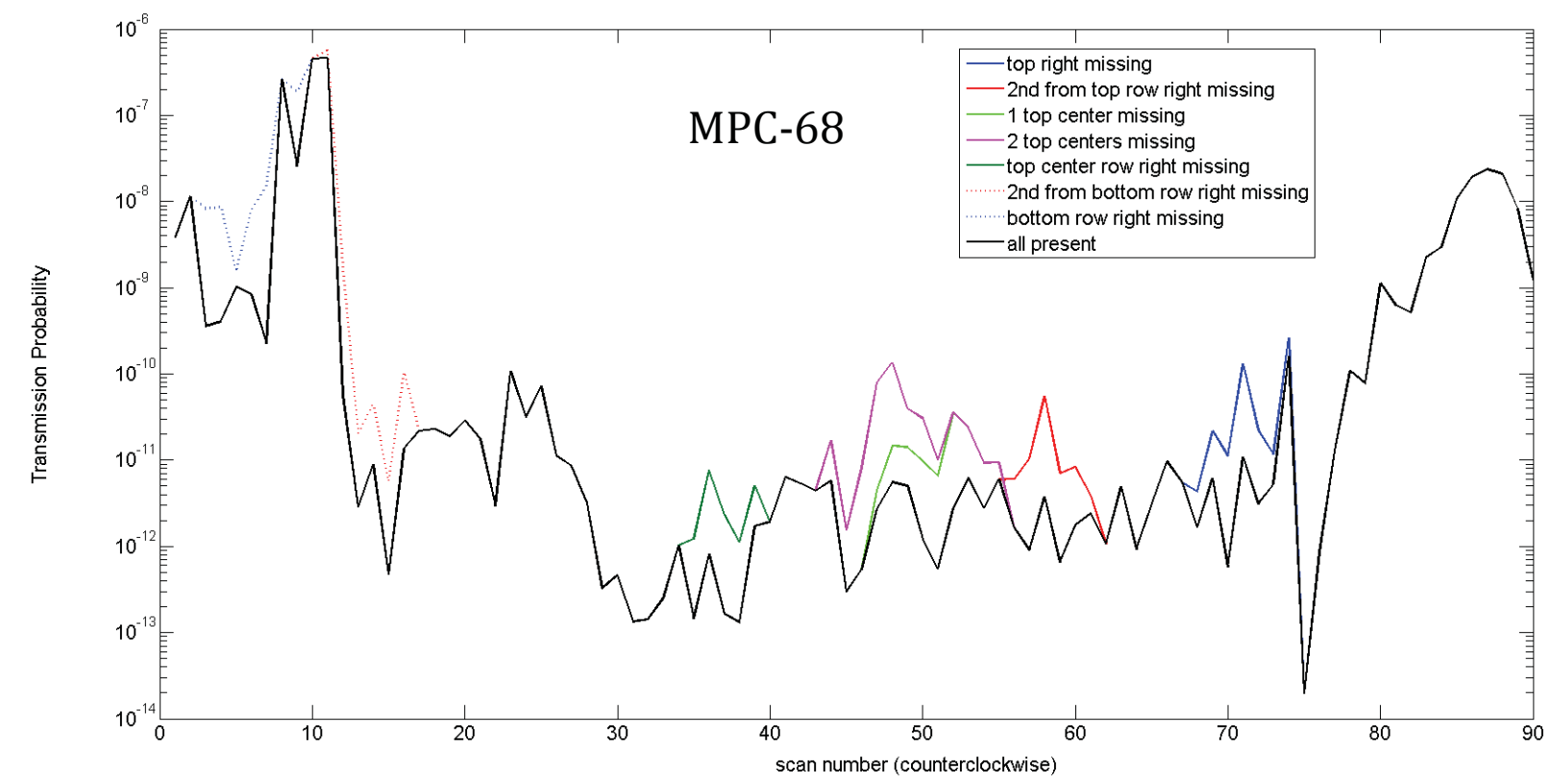

Figure 61. Transmission profiles for $205^{\circ}$ "fan" scans of a HI-STORM MPC-68 cask. The black lines show the transmission probabilities with all assemblies in place. The colored lines give the transmission values with specific assemblies removed as indicated in the legend. 
The analytical results shown in the figures above were validated by MCNP radiation transport calculations for the representative "fan" scan of a HI-STORM MPC-24 cask shown in Figure 62. In the MCNP calculation an $8 \mathrm{MeV}$ beam with 30\% energy spread and $1.75 \mathrm{mrad}$ half-angle was transported through the locations shown. A flux-estimating point detector was placed in the beamline about $5 \mathrm{~cm}$ outside the wall of the cask. This type of detector, along with use of particle splitting and limiting the geometry of the cask to only $60 \mathrm{~cm}$ in height, allows for reasonable computation time for such a highly attenuating model. The reduction in cask height is justified by the very low probability that a photon would scatter far into the cask and then scatter back to the detector. Signal photons are of primary interest.

For most lines through the cask, the analytical values agree well with the MCNP calculated ones. Several analytical data points are slightly above the MCNP simulations, i.e., the transmission is slightly underestimated in the analytical calculation, and only for one beam is the analytical transmission probability larger than the MCNP value. For the purpose of the analysis presented here, the analytical estimates are therefore sufficiently accurate. The simulations confirm the ability of a scanned, narrow-divergence MPS beam to verify occupancy in the assessed dry storage casks. 

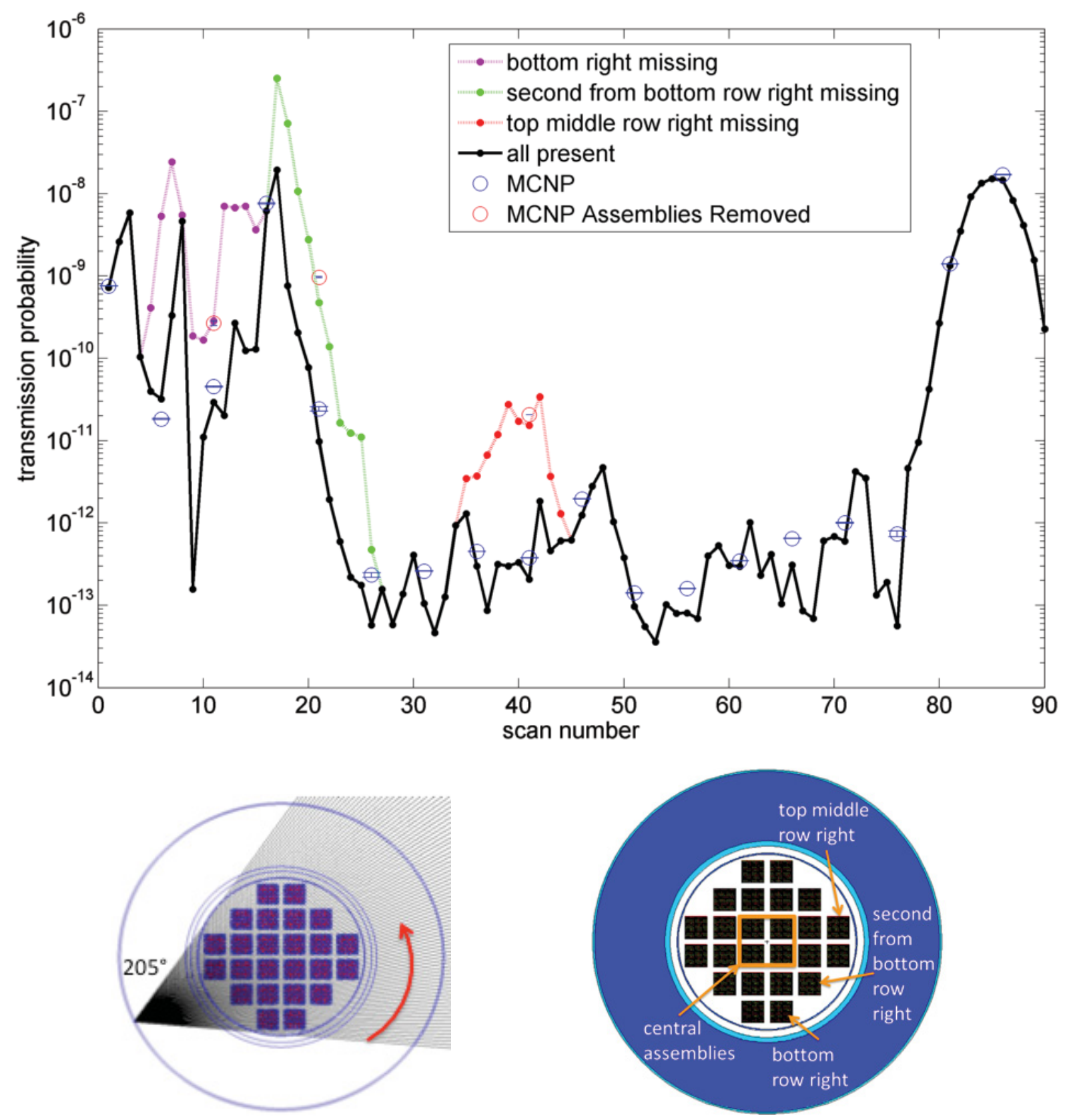

Figure 62. Validation of analytical calculations with MCNP simulations. Analytical calculations for transverse "fan" scan of a HI-STORM MPC 24 cask are shown as black (all assemblies in place) and colored data points. The MCNP results are indicated by the open blue circles (all assemblies in place) and by open red circles for beams through an unoccupied assembly location. 


\section{II.5.2.4. Impact of and Requirements for MPS Systems}

Re-verification of a spent fuel dry-storage cask by measuring the transmission profile is a prime example of how an intense pencil beam of $\mathrm{MeV}$ photons can be used to penetrate very thick objects. Verification of the presence of assemblies or even single pins can be done in a straightforward way by measuring the longitudinal transmission through the cask while scanning a narrow-divergence beam across it. Such a scan could be conducted with photon beam fluxes of $1 \times 10^{11} \mathrm{ph} / \mathrm{s}$ in measurement times on the order of hours even for casks with very thick lids and bottom plates.

In a transverse scan, the transmission probabilities vary greatly across the cask. The transmission is lowest through the center but does not fall much below $1 \times 10^{-12}$ for either of the two types of casks considered here when an optimized source orientation in respect to the assembly pattern is used. A missing assembly increases the transmission by roughly an order of magnitude, as can be seen for the transmission profile in Figure 63. Such an increase is easily detectable and the measured transmission profile enables the determination of unoccupied assembly positions by comparison with the expected profile for a fully loaded cask. A beam flux of $5 \times 10^{12} \mathrm{ph} / \mathrm{s}\left(5 \times 10^{8} \mathrm{ph} /\right.$ pulse, $10 \mathrm{kHz}$ repetition rate $)$ would result in at least $1 \mathrm{count} / \mathrm{s}$. Assuming a minimum of 100 counts per beam chord, a full cask scan consisting of 90 beam chords could be performed in an acceptable measurement time of less than three hours.

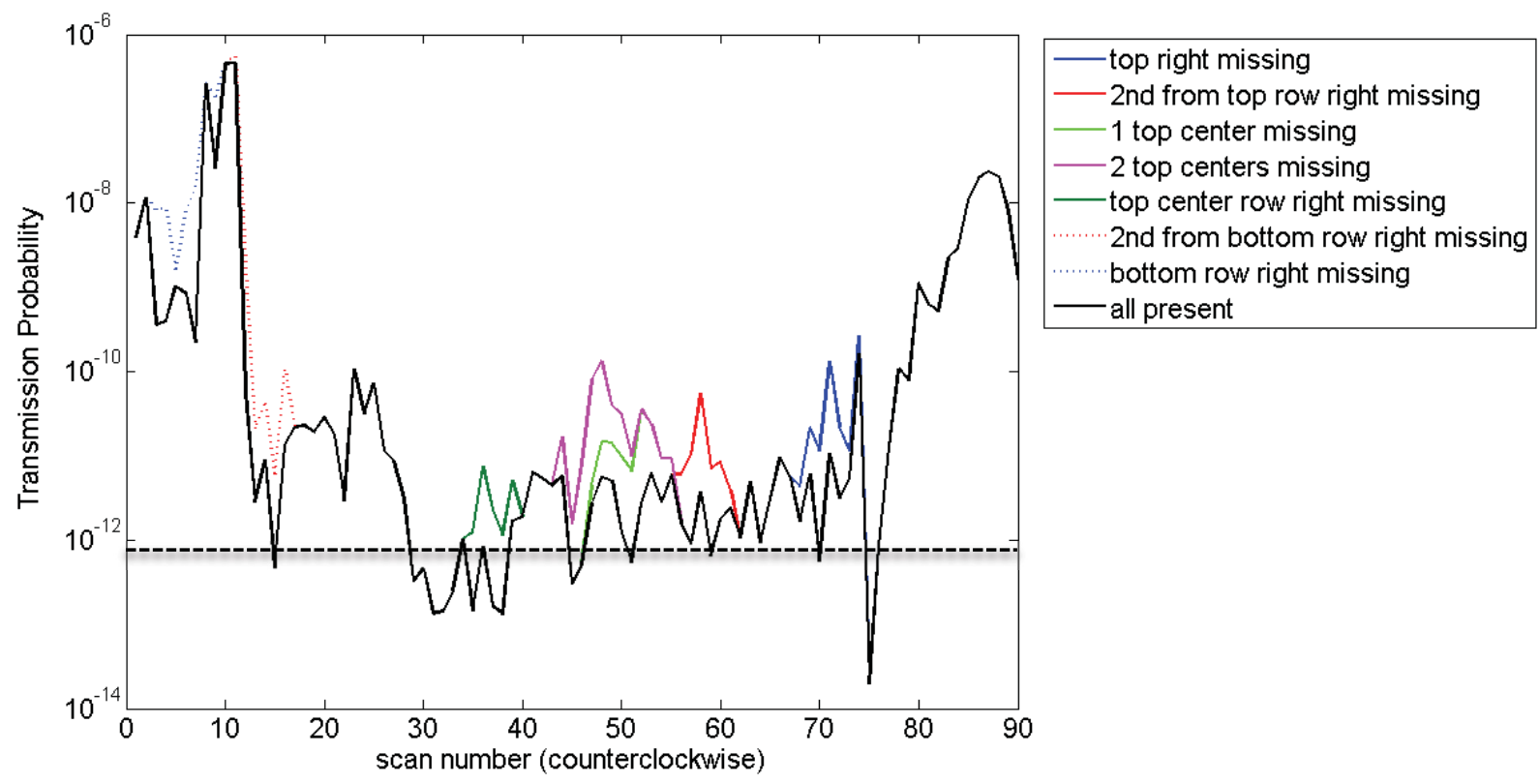

Figure 63. Analytically calculated scan with 90 beams of a HI-STORM MPC 68 cask. The black data points show the transmission probabilities for the 90 lines with all assemblies in place. The colored data points give the transmission values with specific assemblies removed as indicated in the legend. The lines connect the data points. The source position is at 205 degrees.

This application requires a photon source that produces an intense, low divergence photon beam in the 6 to $8 \mathrm{MeV}$ energy range. A fairly large energy spread (20-30\% FWHM) that maximizes the number of photons/pulse is acceptable. The beam diameter should be significantly smaller than the assembly width, which suggests an angular divergence of several mrad. A lower divergence of $\sim 2 \mathrm{mrad}$ is required for resolving individual fuel pins in lengthwise measurements through the cask. For transverse transmission measurements a high yield of $>1 \times 10^{12} \mathrm{ph} / \mathrm{s}$ is 
required to overcome the main challenge, which is low transmission through the massive cask. The source should be pulsed with nanosecond or shorter pulse length to enable efficient detector gating for discriminating against the passive background from the spent fuel in the cask. These requirements could be met by a Thomson source operating at $>10 \mathrm{kHz}$ and $5 \times 10^{8} \mathrm{ph} / \mathrm{shot}$. Importantly, the MPS needs to be transportable and sufficiently compact to be set up next to a dry-storage cask and to be moved as needed.

The dry-storage cask verification capability provided by a Thomson source with the above characteristics could fill a gap, as currently no satisfactory method for verifying assembly occupancy exists. Two potential alternative techniques are being explored. The ability of the Compton Dry-Cask Imaging Scanner (CDCIS) [111] developed at INL to detect empty vs. full storage positions was demonstrated for a Westinghouse MC-10 cask. However, the scanner was not able to distinguish empty vs. full positions for casks in Doel (Belgium), which was likely due to the presence of a thick steel shield over the lid of the cask, which introduced too much scattering. Preliminary results from first tests of a cosmic-ray muon imaging system [112] that is currently under development have indicated that missing fuel bundles can be detected after 100 hours of exposure. It is not yet clear how much the performance of that system can be improved, and capabilities will need to be compared as both systems develop. Furthermore, scanning a cask from a few different directions (multiple views) using a photon source may allow additional evaluation of loading structure and material distribution inside the cask, which may provide advantages over other methods. The ultimate impact of an MPS-based system will likely depend on the efficacy of other techniques, such as muon imaging, that are under development.

\section{II.5.3. Assay of Spent Fuel Assemblies}

\section{II.5.3.1. Introduction}

The incorporation of new nondestructive assay (NDA) systems into safeguards approaches for spent nuclear fuel (SNF) assemblies has been examined in a report by Abhold et al. commissioned by the Office of Nuclear Safeguards and Security, NNSA Office of Nonproliferation and International Security [119]. The report gives "two primary concerns related to spent fuel assemblies: One is the potential for the removal or substitution of individual pins from an assembly. The second one is accountancy when the assemblies are chopped up and dissolved or melted." The report identifies applications with the greatest relevance for the examined NDA measurement systems being developed under the Next Generation Safeguards Initiative (NGSI) for determining Pu and fissile content [120]. A MPS-based system that measures the amount of specific isotopes in the SNF could address several of those applications in a new way including (taken from the report by Abhold et al. [119]):

- Reestablishment of inventory after loss of continuity of knowledge (COK).

- "Fingerprinting", i.e., acquiring an SNF assembly "signature". An unchanged signature at a later time would provide assurance of the assembly integrity.

- Establishing input accountancy for pyroprocessing. It is not yet clear whether pyroprocessing will have an input accountability stage. Without it, measurement of the plutonium content of the spent fuel would be needed for establishing input accountancy.

- Establishing disposition accountancy by verifying the $\mathrm{Pu}$ content independently from operator declarations.

- Reconstruction of Reactor Operating History. An inconsistency of the measured spent fuel isotopic composition with the declared reactor operating history can indicate undeclared production of plutonium. 
Any new approach must fit within the existing operating process and inspection regime and measurements on spent fuel generally need to be performed at a storage pool or in a hot cell.

It should be noted that the spent fuel assembly assay is currently not considered a priority in international safeguards (discussion with DNN R\&D stakeholders) and is therefore identified as having low priority for further study.

\section{II.5.3.2. Background and Current Status}

NDA measurement instruments and techniques for determining $\mathrm{Pu}$ and fissile content in spent nuclear fuel assemblies have been investigated in the NGSI SNF project [119] and other studies [120,121]. The NGSI developed instruments primarily measure Pu-effective, a weighted sum of all fissile isotopes, from which the $\mathrm{Pu}$ content has to be inferred using additional information or combining results from several instruments. High accuracy measurements are difficult due to the variation of assembly characteristics with reactor history and the indirect nature of the techniques. NDA of Pu mass with uncertainties on the order of $1 \%$ has not yet been demonstrated. Uses of NDA systems in other applications, such as "fingerprinting" or verification of declared quantities, are being researched and tested.

The use of NRF for spent fuel assay and other applications has been researched in recent years and modeling has shown that transmission NRF could directly measure the amount of specific Pu isotopes (or other isotopes) in spent fuel $[122,17,21]$. However, photon sources with capabilities that would allow to achieve required measurement uncertainties in acceptable times and of a size that would allow integration into or use in nuclear facilities are not yet available. Future MPSs may fill this gap.

Modeling of NRF measurements on spent fuel assemblies has shown the potential for detecting single pin diversion, [17] but given the advances in instrumentation development made in recent years, pin diversion detection is not considered an application where a MPS would have an impact. While currently used spent fuel verification techniques such as Cerenkov viewers are limited in their capability to detect pin substitution [119], the FORK detector [123] can easily detect a $50 \%$ partial defect but is not sensitive to a single missing pin in a light water reactor assembly. New instruments have recently been developed or are under development. The Partial Defect Verifier (PDET) [124] has been proven to be effective in detecting a few percent of missing pins in an assembly without any fuel movement and operator-provided information. Passive Gamma Emission Tomography is another technique under development with the potential to detect single pin diversion [125].

\section{II.5.3.3. Potential Capabilities and Advantages of MPS-based System}

Transmission NRF directly measures the areal density of a specific isotope along the beam's path independently of other materials present or of the structure of the object. By scanning the beam through the object-possibly by rotating and/or translating the assembly-the isotope specific (Pu-239, Pu-240, Pu-242, U-235) content could be determined for the assembly or at specific locations to address the applications listed below.

- The direct measurement of $\mathrm{Pu}$ isotopic content could provide high-quality data for the reestablishment of inventory after loss of CoK.

- The measurement of $\mathrm{Pu}-239 / \mathrm{Pu}-240 / \mathrm{Pu}-242 / \mathrm{U}-235$ ratios at a particular assembly position could be an excellent "fingerprint". Another possible way to "fingerprint" an assembly could be to measure the $\mathrm{Pu}-240$ areal density at several locations in the assembly. Because the assembly burnup is not uniform and depends on the history of the assembly in the reactor, the $\mathrm{Pu}-240$ concentration can vary by $10 \%$ or more across the assembly. The $\mathrm{Pu}-240$ measurement would likely be considerably faster than the measurement of isotopic 
ratios because the $\mathrm{Pu}-240 \mathrm{NRF}$ resonances are significantly stronger than those of $\mathrm{Pu}-239$ [126].

- The direct measurement of $\mathrm{Pu}$ isotopes in spent fuel could provide needed input accountancy for a pyroprocessing plant. This may best be done after the assemblies are shredded and placed into thin, planar baskets before going into the refiner vessel.

- A direct $\mathrm{Pu}$ measurement in spent fuel assemblies could also provide disposition accountancy with relying on operator declarations.

- Measured $\mathrm{Pu}-239 / \mathrm{Pu}-240 / \mathrm{Pu}-242$ ratios could provide valuable information about the reactor operating history and could detect inconsistencies between the measured ratios and the declared operating history.

\section{II.5.3.3. Impact of and Requirements for MPS Systems}

NRF transmission measurements on spent fuel require a photon beam with selectable energies between 1.7 and $2.5 \mathrm{MeV}$, a high spectral density at the resonance energy for a sufficient $\mathrm{NRF}$ count rate, and a narrow energy spread to minimize the background. Thomson scattering sources have the potential to meet these requirements. Because these sources are pulsed and may operate at repetition rates that are too low to be compatible with the use of spectroscopic detectors, the concept of a calorimetric detector has been developed that measures the deposited energy and not single photons [21]. When using a calorimetric detector, the measurement time scales approximately inversely with the energy spread squared for a constant number of photons per pulse, and a narrow energy spread is key for transmission NRF measurements.

Results from previous modeling studies provide reference points for estimating photon source requirements. For example, the time for measuring a $0.45 \% \mathrm{Pu}-239$ fraction in a $17 \times 17$ PWR assembly with $3 \%$ statistical uncertainty was estimated to be several hours assuming a 17 $\mathrm{eV} \cdot \mathrm{b}$ resonance cross section and a photon beam of $3 \times 10^{11} \mathrm{ph} / \mathrm{s}$ with an energy spread of $\sim 0.1 \%$. $[17,21]$ The time for measuring the $\mathrm{Pu}-240$ fraction was $\sim 100$ times shorter because of a larger resonance cross section $[17,12]$. It should be noted that the $\mathrm{Pu}-239$ resonant absorption cross section is not well known introducing a large uncertainty into the above estimate. The uncertainty in NRF measurements is usually dominated by the counting statistics and thus improves with measurement time. For measurements that significantly exceed the performance of alternative approaches and are of acceptable duration in the SNF assay context, the critical photon source requirements are an energy spread on the order of $0.1 \%$ FWHM and a photon yield on the order of $10^{12} \mathrm{ph} / \mathrm{s}$. Such capabilities are beyond anticipated performance for near term MPSs.

For use at an existing nuclear facility, the MPS must fit within the available space. The size of a transportable MPS that fits on a small or medium truck may be acceptable. New facilities such as a new pyroprocessing plant could integrate an MPS-based system into their design.

NRF potentially offers unique capabilities for safeguards but substantial advances in MPS technology are needed to enable NRF measurement techniques that significantly enhance existing capabilities. Results of previous modeling studies of spent fuel assembly assay indicate that a MPS with a photon yield on the order of $10^{12} \mathrm{ph} / \mathrm{s}$, an energy spread around $0.1 \%$, and a beam divergence of $<1 \mathrm{mrad}$ could significantly strengthen SNF assembly assay capabilities. Because the development of a source that meets these challenging requirements is a long-term prospect and because SNF assembly assay was at the time of this assessment not a priority in international safeguards, this application is identified as something to be considered at a later time. 


\section{II.5.4. Materials Characterization in Accident Scenarios}

\section{II.5.4.1. Introduction}

The Fukushima nuclear accident introduced a new material accountancy challenge, as the melting of fuel caused a loss of continuity of knowledge, and existing safeguards protocols no longer apply. The Japanese Atomic Energy Agency (JAEA) is currently studying new methodologies for regaining accountancy and determining the amount of plutonium in the fuel debris. The characterization of nuclear material after damage in an accident poses special challenges. Damaged and melted fuel may have lost its assembly structure and may be mixed with debris and thus very heterogeneous in composition and density. In order to adequately account for all material, an NDA method is needed that can quantify the main Pu isotopes through the volume of the debris and that is not affected by the composition and structural form of the material. Any technology developed for the assaying of Fukushima fuel debris could have broader applications in material accountancy-in particular, for bulk assaying such as determining total $\mathrm{Pu}$ in waste drums/tanks or in vitrified materials.

\section{II.5.4.2. Background and Current Status}

R\&D is currently being performed by the JAEA towards the goal of assaying Fukushima melted fuel. Traditional neutron based methods or passive measurements can likely not provide this capability due to unknown composition and form of the material to be recovered from the site [127] and put into special canisters for analysis and storage. The containers to be used for holding damaged fuel will likely be similar to the ones used at Three Mile Island. TMI-2 containers could be used for damaged assemblies and other suitable containers for smaller debris.

Transmission NRF and has been identified by JAEA researchers as the most promising method to precisely assay thick samples of shielded fissile material of unknown composition [128]. In transmission NRF the areal density of the isotope along the beam's path and in this way the isotopic content is measured directly and independently of distribution and form of the materials in the container. Neutron transmission resonance absorption spectroscopy [129] is also being considered [127]. Both methods measure resonant absorption, either of photons or of neutrons in transmission mode.

In transmission NRF the areal density of the isotope along the beam's path and, therefore, the isotopic content is measured directly and independently of distribution and form of the materials in the container.

$R \& D$ is in progress in Japan on a fixed-facility photon source of approximately tens of meters scale to enable NRF measurements. A $350 \mathrm{MeV}$ energy recovery linac (ERL) has been proposed for generating up to $2.2 \mathrm{MeV}$ photons via Thomson scattering [19]. A very high pulse frequency, in the range of $100 \mathrm{MHz}$, allows the use of spectroscopic HPGe detectors, which makes it possible to use a photon beam with a much broader energy spread than is required for an NRF system employing a calorimetric detector [130].

\section{II.5.4.3. Potential Capabilities and Advantages of MPS-based System}

A MPS is likely the key component for enabling material accountancy in accident scenarios. Transmission NRF, which has been identified as the most promising method for this application, relies upon a MPS that generates a photon beam with the required intensity and narrow energy spread. The proposed energy-recovery linac (ERL)-based photon source [19] is a complex accelerator with a large footprint that requires fixed-facility installation but provides the advantage of a very high pulse frequency. Thomson sources considered in this study, either based on a conventional electron linac or a laser plasma accelerator, are much smaller than the 
proposed ERL, which could greatly increase the ease and flexibility of implementing an NRF measurement system.

\section{II.5.4.4. Impact of and Requirements for MPS Systems}

MPS requirements depend foremost on how well the damaged fuel needs to be characterized, i.e., on the required uncertainties for the measurement of the Pu isotopics. The MPS performance requirements depend further on the properties and packaging of fuel debris or damaged assemblies. In addition, uncertainties in NRF measurements are dominated by counting statistics and can thus be reduced by measuring for longer times. Because cleanup operations may take years, rather long measurement times are likely acceptable and could ease MPS requirements.

Assuming that the size of the damaged fuel objects to be assayed are comparable to an assembly, that the required a measurement uncertainty for the main Pu isotopes are in the few percent range, but that longer measurement times could be acceptable, the MPS requirements will be somewhat less demanding than for spent fuel assembly assay discussed in section 5.2, i.e., a photon yield on the order of $10^{11-10^{12}} \mathrm{ph} / \mathrm{s}$ and an energy spread of around $0.1 \%$ will be sufficient. This narrow energy spread is important for MPSs that operate at $\mathrm{kHz}$ repetition rates to allow use of a calorimetric detector to measure the NRF signal. As mentioned above, $\mathrm{MHz}$ repetition rates allow for a broader energy spread. Smaller object sizes could be characterized using significantly lower fluxes.

Because the development of a source that meets the challenging requirements of assaying damaged fuel and fuel debris is a long-term prospect, and because materials characterization in accident scenarios was at the time of this assessment not considered an urgent problem in International Safeguards, this application, which is closely related to spent fuel assay, is identified as one to be considered at a later time.

\section{II.5.5. Material Accountancy and Pu Monitoring in Pyroprocessing}

\section{II.5.5.1. Introduction}

In pyroprocessing the spent fuel is dissolved in a liquid salt solution from which $U$ and TRU can be extracted by collection of the materials on electrodes for further processing into new fuel. This process is still under development and no production facility exits. In the US, R\&D on electrochemical processing has been pursued for many years at the Idaho National Laboratory. In addition to its R\&D effort, the program is charged with converting the fuel from the Experimental Breeder Reactor (EBR) II and from the Fast Flux Test Facility (FFTF) reactor to a safer form, i.e., extracting U-235 from the highly enriched fuel [131]. While the U-235 is used for fresh fuel, Pu and other TRU stay in the salt solution and are disposed of and vitrified for eventual permanent storage. Efforts are under way in other countries to develop and build electrochemical reprocessing facilities where the TRU will be recovered in addition to $U$ for fresh fuel fabrication $[132,133]$.

Pyroprocessing poses new material accountancy challenges that are distinct from those in aqueous reprocessing. Because pyroprocessing facilities may not have an input accountability tank, there is a need for an input accountancy measurement as the fuel bundles gets cut up and the pieces put into a basket that is then immersed into the salt solution in the refiner vessel [134]. Further needed for accountancy are measurements of the amount of $\mathrm{Pu}$ in the precipitate and removed from the refiner vessel, of the Pu that stays in the solution and is discarded with it, and of the Pu content of the fresh fuel. New NDA instruments and measurement techniques will be needed to meet material accountancy requirements. 
Another important challenge in pyroprocessing is monitoring the concentration of $\mathrm{Pu}$ in the refiner vessel, which is needed for good process control. The measurement uncertainty of the $\mathrm{Pu}$ concentration in the refiner vessel can be larger than in accountancy measurements. Being able to see the $\mathrm{Pu}$ concentration trending up or down is already quite useful. Although this is strictly speaking not a safeguards issue, monitoring $\mathrm{Pu}$ in the refiner vessel could support overall accountancy.

\section{II.5.5.2. Background and Current Status}

As pyroprocessing is still under development and no production facilities exist, the material accountancy challenges have not yet been brought to the forefront. Existing NDA methods and instrumentation developed or under development as part of the NGSI Spent Fuel program may quite possibly not be able to meet the uncertainty requirement of spent fuel measurements for input accountancy of about $1 \%$ or less.

Concentrations in the refiner vessel at the INL facility are currently determined by sample analysis. While this yields very precise results, it is a cumbersome and slow procedure that does not allow process monitoring, as turn-around times can be days or weeks. An in-situ method is therefore a priority.

\section{II.5.5.3. Potential Capabilities and Advantages of MPS-based System}

The same transmission NRF measurement techniques that are discussed above for the assay of spent fuel assemblies can be applied to material accountancy measurements in pyroprocessing, i.e., to the input accountancy measurements on intact or cut-up fuel, and the measurement of $\mathrm{Pu}$ content in the precipitate, in the solution to be discarded, and in the fresh fuel. The same considerations regarding measurement methods and capabilities apply here.

In addition, transmission NRF offers the unique potential to non-destructively monitor $\mathrm{Pu}$ concentrations in the refiner vessel, an important challenge in pyroprocessing, by directly measuring the areal density for a specific isotope along a path through the vessel. The refiner vessel at INL is $1 \mathrm{~m}$ in diameter and $1 \mathrm{~m}$ in height and filled to $\sim 55 \mathrm{~cm}$ in height with the molten salt solution. Given the solution density of $\sim 1.75 \mathrm{~g} / \mathrm{cm}^{3}$, the areal density going vertically through the vessel is $\sim 100 \mathrm{~g} / \mathrm{cm}^{2}$ and of similar value for a horizontal measurement along a path away from the center of the vessel. These areal densities are comparable to those of PWR assemblies and thus measurement parameters can be expected to be in the same range as those for spent fuel assay. Refiner vessels for future production facilities will likely be larger than the vessel at INL. However, current designs feature rectangular shapes in which the spent fuel baskets and the electrodes are arranged in rows. The height of the salt solution and the path length horizontally through the vessel are likely similar to those of the INL vessel, which would allow transmission $\mathrm{NRF}$ measurements of the $\mathrm{Pu}$ concentration in the refiner vessel of future pyroprocessing facilities. Frequent or continuous measurements of the Pu concentrations in the refiner vessel may make separate input accountancy measurements unnecessary, provided they can be performed with sufficient accuracy.

\section{II.5.5.4. Impact of and Requirements for MPS Systems}

The MPS requirements for the spent fuel input accountancy measurements would be the close to those for assaying spent fuel assemblies as discussed in Section II.4.3. For monitoring the Pu concentration in the refiner vessel, the MPS requirements would likely be less demanding and $10 x$ lower beam intensity (of $10^{11} \mathrm{ph} / \mathrm{s}$ ) or a wider energy spread acceptable.

An additional challenge is given by the operation of the refiner in a hot cell environment. The photon beam has to be brought in from the source and out to the NRF detection system through narrow collimators. A Thomson scattering MPS is well suited for this scenario as for such sources 
the required narrow energy spread goes hand in hand with a very low angular divergence, on the order of 0.1 mrad.

The initial assessment indicates that a MPS meeting the challenging requirements, which is a long-term prospect, could potentially enable unique solutions for the monitoring and accountancy needs in pyroprocessing. Because the future of pyroprocessing is not clear, addressing its needs was at the time of this assessment not considered urgent. Therefore, this application, which is closely related to spent fuel assembly assay, is identified as one to be further considered at a later time.

\section{II.5.6. Verification of $\mathrm{UF}_{6}$ Cylinder Enrichment}

Safeguards at uranium enrichment plants require accurate and independent measurements of enrichment in the product, feed, and tail streams. The IAEA has pursued new measurement techniques to address the verification challenges posed by modern centrifuge enrichment plants and developed the concept of an Unattended Cylinder Verification Station (UCVS) to monitor $100 \%$ of material flow. The UCVS will include instrumentation to assay cylinder enrichment, U235 mass, total uranium mass, and re-verification of an "NDA Fingerprint" [135].

Two instruments were developed, the Hybrid Enrichment Verification Array (HEVA) by Pacific Northwest National Laboratory (PNNL) [136] and the Passive Neutron Enrichment Meter (PNEM) $[137,138]$ by Los Alamos National Laboratory (LANL). Initial studies have indicated that both instruments are capable of assaying relative enrichment with a precision meeting the international target values [139] and of interrogating the full volume of the cylinder for verifying U-235 mass. Both methods assume a known U-234/U-235 ratio. While it is not established how firm this assumption holds, both methods can detect U-234/U-235 ratios out of the typical range. Provided the test results are confirmed in field trials, the instruments successfully filled an existing gap in cylinder verification. Lowering the measurement uncertainty to $1 \%$, which is of interest to safeguards (discussion with DNN R\&D stakeholders), would likely require further instrumentation development.

Transmission NRF measurements could, in principle, be used to directly and independently measure the U-235/U-238 ratio and the absolute mass of U-235 in the full volume of a $\mathrm{UF}_{6}$ cylinder. While small cylinders could be penetrated using a MPS with a spectral density at the upper end of the range considered here, measurements on 30B and 48Y cylinders, with several times the areal density of a PWR fuel assembly, would take a very long time or even be impossible. In addition, NRF is an active method, which would complicate unattended operation.

Given the performance of the newly developed UCVS and the challenges NRF methods face in full volume measurements of $30 \mathrm{~B}$ and $48 \mathrm{Y} \mathrm{UF}_{6}$ cylinders a role for an MPS-based system in verification of $\mathrm{UF}_{6}$ cylinders could not be identified.

\section{II.5.7. Verification of Fresh Fuel in Transport Containers}

\section{II.5.7.1 Introduction}

Surplus plutonium is dispositioned as mixed-oxide (MOX) fuel in existing domestic commercial reactors (BWRs or PWRs). Because the fresh, unirradiated fuel contains large quantities of plutonium, the Pu content in mixed oxide fuel varies from $2 \%$ to $30 \%$ [134], and is not sufficiently radioactive to create a "self-protecting" barrier to deter theft, robust safeguards 
and security measures must be in place.

MOX and other fresh fuel is transported from the fabrication facilities to the commercial reactors in special transport containers that hold 2 or 4 PWR assemblies and up to 8 BWR assemblies [140]. The shipping packages have a steel shell $(<0.5$ inch thick) and steel support structures for holding the assemblies in place. The assemblies are surrounded by load foam and $\mathrm{B}_{4} \mathrm{C}$ poison plates are placed between the assemblies.

New methods and instrumentation is being sought for verification of fresh (not just MOX) fuel in transport containers for diversion detection. Partial defects of $50 \%$ of pins need to be detected and a determination of the type of fuel in the transport container is desirable (discussion with DNN R\&D stakeholders).

\section{II.5.7.2. Background and Current Status}

NDA methods are in use for the measurement of $\mathrm{Pu}$ content at MOX fabrication facilities including an unattended custom designed NDA system for monitoring of Pu content in MOX feed canisters. That instrument employs coincident neutron counting in combination with highresolution gamma spectroscopy to derive total Pu mass [134].

DNN R\&D included Verification of MOX Fuel in Transport Containers in its latest call for proposals, and it can be expected that new methods and instrumentation will be developed over the next few years.

\section{II.5.7.3. Potential Capabilities and Advantages of MPS-based System}

A MPS could be employed for radiographing the shipping package and for performing NRF measurements to ascertain what type of fuel is transported and to verify that it is not DU. Many of the considerations discussed in Section II.1, Cargo Screening Verification apply here, and a radiograph could be taken by scanning the transport container with a low divergence ("pencil") beam with excellent contrast. In contrast to the dry-storage cask verification, Section II.5.1, the steel shell of the container is rather thin and can easily penetrated. It should be noted that a bremsstrahlung source could also be used to radiograph the shipping package.

For ascertaining the type of fuel either transmission or backscatter NRF measurements could be used. Transmission NRF could measure through the full volume of an assembly but would require a photon beam with higher intensity and narrow energy spread, the sequential measurement of Pu-239 and U-235, and longer measurement times. A backscatter measurement of $\mathrm{Pu}$ and $\mathrm{U}$ isotopes could be performed with relaxed photon beam parameters, i.e., wider energy spread, in much shorter measurement times but would probe only the outer rows of pins in an assembly.

\section{II.5.7.4. Impact of and Requirements for MPS Systems}

The MPS requirements for radiography are less demanding than for cargo screening (Section II.1), as the fresh fuel shipping packages are significantly smaller than $40 \mathrm{ft}$ cargo containers. The requirements for transmission NRF are on the same order of magnitude than those for SNF assembly assay in Section II.5.2. This would allow measurement times on the order of minutes given that the $\mathrm{Pu}-239$ or U-235 fractions in fresh fuel are a factor of $\sim 10$ higher than in spent fuel and the required measurement precision significantly lower. For backscatter NRF beam intensity and energy spread requirements would be less significantly lower than for transmission NRF.

While a MPS-based system as outlined above would be capable of verifying fresh fuel in transport containers with high confidence, it is an active interrogation system with considerable complexity and cost that does not fit well into safeguards operation. Because one can reasonably expect that verification systems based on passive NDA techniques or other easier to operate 
systems will be developed over the next few years, this application is not identified as a priority for further assessment, but it could be reconsidered at a later time. 


\section{II.6. Emergency Response}

\section{II.6.1. Introduction}

Detection and neutralization of the threat posed by an illicit nuclear device is an application area that may involve several steps and a corresponding range of techniques. These steps may include Search scenarios to detect nuclear material, typically using gamma and neutron radiation, but possibly also including high-explosives detection and radiography. Stabilization of a detected device involves performing diagnostics on a device using all available instruments and techniques, such as radiation detection and identification, high explosives identification, and radiography. It also includes SNM characterization such as determination of the enrichment of shielded uranium and of the chemical form of Pu. Render Safe of a device may also involve radiation, highexplosives detection and identification, and radiography. Where a radiograph shows a region of interest within the container, detailed diagnostics are of interest to unambiguously identify and characterize SNM. Active interrogation systems are still in the research and development stage and are not yet deployed. They could rely on the characteristic signatures of fission, namely prompt neutron and delayed gamma and neutron emissions or on nuclear resonance fluorescence (NRF).

Emergency Response may benefit from almost the full range of potential MPS-based techniques assessed in Sections II.1 Cargo Screening (radiography of shielded objects, as well as both photofission and NRF material identification), II.2 Detection of Hidden SNM (photofission detection of shielded material), and III.3 Treaty and Dismantlement (high resolution radiography and NRF for material identification). In general however, Emergency Response requires smaller and more mobile systems than other applications, which may require longer-term MPS development.

This section briefly reviews the status of Emergency Response techniques, followed by a brief assessment of the potential benefit from the application of MPS based techniques. The assessment of benefit is based on the results of sections II.1-3 because of the similarities in techniques to those used in other areas. A separate simulation study was beyond the scope of the project.

\section{II.6.2. Background and Current Status}

Several modalities are utilized by the emergency response teams to assess a device, and to identify the best way to disable it. An inspection may start with taking a low dose radiograph with a $450 \mathrm{keV} \mathrm{x}$-ray machine in order to look for switches, wires, capacitors, etc. that may be used in a booby trap. At this initial state, imparting a low dose is important in order to avoid the possible triggering of a trap. In a second step, higher quality imaging is done with a $450 \mathrm{keV} x$-ray machine to identify components and to support an analysis before dismantling and rendering the device safe. High-energy radiography with a 3-4 MeV bremsstrahlung beam generated by a betatron may also be performed to image and characterize the SNM configuration and possibly other structures. Desired improvements include instrumentation for taking higher quality radiographs at lower doses to the object and with better materials identification, and enhanced SNM characterization.

Active interrogation methods, while currently not in use, are of strong interest and are currently being investigated to demonstrate that they are safe and add valuable information. This includes the use of a neutron generator and gamma sources. A high-power laser for cutting and disabling components is currently being added to the set of instruments.

Mobility is a high priority. The equipment and instrumentation generally fit into air cargo containers for quick transport to the site. The instrumentation should be packaged in boxes that can be carried by two persons to where it is needed. Multiple boxes may be used for one 
instrument. It has been noted that pre-positioning of larger equipment at a few locations around the country is in progress, and that in one case a truck-based instrument was used.

\section{II.6.3. Potential Capabilities and Advantages of MPS-based Systems}

A monoenergetic photon source could potentially enhance several emergency response capabilities. Several photon source characteristics could constitute improvements: selectable photon energy from $<1 \mathrm{MeV}$ to $>10 \mathrm{MeV}$, narrow energy spread, low divergence ("pencil") beam, and submicron size of the photon emission volume (source size). Here, we briefly describe the key potential benefits, referencing the section in which detailed description is provided. An overview of MPS advantages for imaging and radiography is presented in Section I.1, Methods and Signatures. Simulations conducted demonstrate several important benefits:

- Imaging with monoenergetic photons benefits from the ability to select photon energy for optimal penetration and contrast based on the device configuration (II.1, Screening and Interdiction). This can reduce dose by a factor of 3-4 for a monoenergetic source having similar emission angle to current bremsstrahlung based systems.

- Imaging with monoenergetic photons at two or more different energies improves Zdetermination and thus helps to identify SNM, explosives and other components (II.1, Screening and Interdiction). Contrast in $\mathrm{Z}$ is increased by several-fold for the assessed configurations by use of dual-energy MPS ratios. This can enable effective discrimination in cases that are not currently accessible, and/or reduce the dose required to achieve discrimination in cases that are currently accessible by severalfold.

- Higher quality images with better contrast are enabled at significantly lower doses by scanning the object with a narrow divergence "pencil" beam due to elimination of the scatter contribution to the image. The quantitative improvements are described in Section II.1, Screening and Interdiction. For vehicle sized objects, dose reductions can exceed an order of magnitude compared to cone beam sources (a factor of a few relative to fan beams), and images can be obtained in cases that are not otherwise accessible. By taking advantage of spot-by-spot dose modulation, an additional severalfold dose reduction could result from scanning the container with a pencil beam. These benefits compound with those from control of photon energy.

- Improved spatial resolution can be achieved by taking advantage of the submicron size of the photon emission volume that is typical for a Thomson source. As detailed in Section II.4, High Resolution Imaging for Stockpile Stewardship, micron-scale resolution is possible. This could lead to better identification of components before disassembly.

Active interrogation methods are also improved and/or enabled by use of monoenergetic photons because a much larger fraction of the photons can be delivered on resonance. A general overview of active signatures is presented in Section I.1. Methods and Signatures.

- Photofission can be excited using an MPS with reduced dose compared to a bremsstrahlung beam. Photon energies $>5 \mathrm{MeV}$ can induce fission, and the cross section peaks near $14 \mathrm{MeV}$. Dose reduction depends on allowable energy. If photoneutron production is not a concern, energies near $13-14 \mathrm{MeV}$ are appropriate and dose reductions from use of an MPS are a factor of 3-4× (Section II.2, Detection of Hidden SNM). If energies are restricted to $<9 \mathrm{MeV}$ to avoid photoneutrons, the dose reduction is much greater-in the range of 50× (II.1, Screening and Interdiction). As with radiography, use of a narrow-divergence beam provides significant additional dose reduction, which is configuration dependent, by concentrating the photons on the area of interest. The results inform the size of a shielded object within which absence could be determined, and indicate that such a measurement could be realistic in objects at the $\sim 1 \mathrm{~m}^{3}$ size range 
using the most suitable source identified. While for single materials it may be possible to use ratios of multiple MPS energies to infer material type, but for multiple materials of unknown order it is not clear this is possible (II.3, Treaty/Dismantlement Verification).

- NRF measurements could be performed using an MPS at selected energies to gather isotopic and elemental information to identify and characterize the SNM, high explosives, and other components. Because the NRF linewidth is very narrow, the dose reduction due to an MPS scales with its energy spread and can easily exceed an order of magnitude. Because off-resonance photons also contribute to detector saturation, MPSs also typically result in much shorter times to detection (in many cases enabling measurements impractical with broadband sources). Simulations indicate the ability of an MPS-based system to identify material in a variety of cases, typically in minutes. Identification of shielded material in container-size objects is described in Section II.1, Cargo Screening, and detailed characterization of both warhead-relevant and small quantities of material is described in Sections II.3, Treaty/Dismantlement Verification, and II.4, Stockpile Stewardship.

A notable advantage of an MPS-based system may be that it could replace several individual instruments: a betatron for higher-energy (3-4 MeV) imaging, an x-ray source (450 keV) for highresolution imaging, and a separate active interrogation system to identify SNM and characterize its configuration. A laser-based MPS may also replace laser-cutting equipment.

\section{II.6.4. Impact of and Requirements for MPS Systems}

The results indicate that a MPS could provide important capabilities for lower-dose radiography, for very high-resolution radiography to identify components at the micron scale, and for secondary identification of material using NRF or photofission. As identified above, any monoenergetic source can reduce radiography dose by a factor of a few (e.g., nuclear reaction sources). Realizing further dose reduction as well as high resolution and secondary identification prioritizes MPSs with adjustable energy, with narrow-angle beams and with small emission spot size (e.g., Thomson sources). Repetition rate and flux could be relaxed relative to cargo applications due to lesser need for throughput. At a repetition rate of $1 \mathrm{kHz}$ the time for one image would be on the order of a minute. Photon energy and beam intensity, i.e., number of photons per pulse, must be quickly variable and well controlled. The beam intensity required for radiography and photofission would be moderate unless substantial shielding would need to be penetrated. NRF measurements of light, heavy and fissile isotopes require photons of selectable energies from $1.7 \mathrm{MeV}$ (U-237) to $\sim 7 \mathrm{MeV}$ (oxygen) corresponding to nuclear resonance energies. Beam intensity and energy-spread requirements would be similar to those for warhead confirmation given in Section II.3.1. In order to fully assess the potential capability enhancement to emergency response that may be provided by a MPS, detailed modeling of measurements on configurations that reflect realistic design features and properties of a nuclear device or a radiological dispersal device is required, which was beyond the scope of this study.

Small MPS size is a priority, which may make this a later application to be addressed. Size and weight of the MPS must meet emergency response needs as noted above, and it must be setup in the field in relatively short times and possibly cramped spaces. Substantial engineering advances, beyond those envisioned for the next 10 years, are needed to reduce MPS technologies to an acceptable size. An exception may be cases where pre-positioning of larger equipment at a few locations around the country may facilitate the use of larger equipment. In these cases, shorterterm applications may be available. 


\section{Related Applications}

This report and project focused on nonproliferation applications of MPSs as identified in the preceding sections. In some cases, dual use was identified important as part of these sections, as in the example of DHS where a system is desired which can address both DNDO missions to detect SNM and CBP missions to detect contraband and other non-SNM materials. Additionally, our discussions with laboratory and agency subject matter experts identified applications outside of nonproliferation which would benefit from similar sources and which may contribute to motivation for their development. These include nuclear waste characterization, nuclear fuel characterization, and also applications outside the nuclear space, such as industrial nondestructive analysis (NDA) or medical imaging.

Characterization of legacy waste or accident material was identified as important both domestically (e.g., Hanford site) and internationally (e.g., Fukushima). Both drum and bulk storage containers are of interest. Radiography can identify high-Z areas in waste drums [141]. The photofission and NRF signatures identified can be used to identify isotopes. NRF methods can provide quantitative assay of spatially resolved isotopic concentrations. This could have a very significant impact on disposal costs for waste. Reflecting this need, JAEA is considering development of a fixed-facility Thomson MPS [19] aimed at characterization of Fukushima material, and a prototype machine is in operation at KEK.

Quality control of fresh fuel rods and inspection of spent fuel rods are important in the nuclear industry. High-flux, narrow energy-spread Thomson sources offer unique capabilities to address this problem [141] [142]. A radiograph of a fuel rod can show density defects but not variations in isotopic concentrations. In contrast, an image obtained in transmission NRF using a near-monoenergetic beam centered on the ${ }^{235} \mathrm{U}$ resonance at $1.733 \mathrm{MeV}$ will show enrichment variations and their magnitude. The small photon emission volume and low angular divergence of a Thomson source could make it possible to achieve excellent, sub-millimeter spatial resolution.

High flux Thomson MPSs offer new opportunities for generating photonuclear data. Perhaps most important to nonproliferation applications could be the availability of a transportable source (even if of more modest performance) that could be brought to a nuclear lab for measurements on material that is difficult to transport. Future sources may also provide photon beams with intensities and spectral densities that exceed capabilities of existing facilities by orders of magnitude. Such intense, near-monoenergetic sources will allow new nuclear physics experiments such as detailed NRF and photofission studies [143].

Beyond nuclear applications, the improved signal and less harmful radiation dose from MPSs has broad potential to benefit other fields that currently use X-ray machines, including industrial NDA and medical imaging. For industrial NDA, thick objects must be penetrated, requiring similar energies as discussed under Stockpile Stewardship (section II.4) and Screening (section II.1). Very small spatial scales are often important, which makes use of small emission spot sizes and narrowly collimated beams important. Industrial applications already have strong commonality with stockpile measurements using current photon sources, and in many cases the same sources and detectors are used. MPS capabilities developed would similarly be cross-applicable. As detailed in Section II.4, High Resolution Imaging for Stockpile Stewardship, micron-scale resolution is possible using Thomson MPSs. This could lead to better identification of structures or defects.

Medical imaging could benefit strongly from reduced dose, which would allow X-ray and CT imaging to be conducted more routinely. As in other radiography applications (see Section II.1), some dose reduction is provided by selecting source energy and narrow divergence can be used 
to further mitigate scattering contributions to image contrast degradation and/or to reduce the dose required to achieve a given image quality. Both would improve diagnostic utility of the images. The high $\mathrm{Z}$ contrast resolution and material discrimination that MPSs could make possible could enable fine distinction of different tissues (e.g., allowing earlier cancer detection), or improved imaging of soft tissue in the presence of bone (which is currently challenging). At the same time, improved spatial resolution is possible with MPSs such as Thomson sources, which have a small emission spot size (as detailed in Section II.4). Sub-MeV energies are appropriate due to the relatively thin object size, and a separate simulation study should be conducted to quantify benefit in this regime.

The potential for widespread MPS use in a range of applications may significantly add to the long-term utility of such sources for nonproliferation applications by providing a broad user base that can help drive development once capabilities are demonstrated and a first transportable source is developed. 


\section{Comments on Nuclear Forensics Applications}

Potential applications for monoenergetic photon sources in relation to nuclear forensics fall into two categories, namely, pre-detonation and post-detonation. Pre-detonation nuclear forensics involves the analysis of nuclear materials, and associated packaging of these materials, to attempt to determine historical attributes including sources of raw materials, methods of manufacture, places of manufacture, places and conditions of storage, modes of transportation, and other characteristics. While timely analysis is always desired, pre-detonation forensics analysis often take place over time scales of weeks. Analytical tools include traditional forensic analysis methods such as visual examination, fingerprinting, and chemical analysis, such as mass spectrometry. Nuclear analyses are also performed including gamma-ray spectrometry, alphaspectrometry, and the use of radiochemistry to augment these techniques. It is not clear what advantages, if any, an MPS source would bring to this field over the currently-deployed techniques. The primary strengths of MPS interrogation including energy specificity, dose reduction, penetration of thick shielding, and the ability to analyze samples without preparation are not at this time seen as strong differentiators for this area and hence no clear benefit is identified.

Post-detonation nuclear forensics involves the analysis of debris and aerosol particles following a detonation, with the aim of identifying the relative intensity of fission daughter products in the debris in order to inform subsequent analyses characterizing the detonation event. The current state of the art in this area involves the collection of field samples and then the transfer of these samples to radiochemistry laboratories where traditional radiochemical analytical methods and tools are employed. The isotopic complexity of these samples, along with other technical and non-technical reasons, severely restricts the potential applicability of an MPS source in this application area. 


\section{Summary of Application Impact and MPS Development Requirements}

Simulations have been presented that evaluate MPS benefit and parameter requirements for a set of nonproliferation and related applications identified and prioritized through discussions with laboratory subject matter experts, DNN R\&D, and its stakeholders. The high-priority applications analyzed were: cargo screening, detection of hidden SNM, warhead/dismantlement verification, and nuclear fuel cask scanning. The initial assessment and the simulations are summarized for each application area in the Executive Summary. The simulations detail the performance achievable for each application and the required MPS parameters including source tradeoffs and constraints (e.g., bandwidth, intensity, pulse structure, and beam angular spread). Simulations focused on the conventional signatures of radiography, photofission, and NRF to enable comparison with present methods and evaluation of benefit. Full system performance was considered to evaluate the impact that monoenergetic sources would have. This included natural and induced background radiation, beam propagation, signature generation and detection, penetration limits, and radiation dose to target and operators. A high-level preliminary evaluation only was presented for a broader set of applications.

Significant potential benefit was indicated for each high-priority application. Common source requirements include production of monoenergetic photon beams at energies ranging from 1.5$15 \mathrm{MeV}$, and with typical fluxes on target in the range of $10^{10}$ photons per second for initial capabilities to $10^{12}$ photons per second for high performance applications. Pulsed sources should have repetition rates in the $\mathrm{kHz}$ range with $10^{7}$ to $10^{8}$ photons per shot to enable initial capabilities (e.g., low-rate cargo scanning). Repetition rates in the $20-50 \mathrm{kHz}$ range would enable high performance, (e.g., cargo scanning at $\sim 80 \mathrm{~cm} / \mathrm{sec}$ rates).

Replacing a bremsstrahlung source with a monoenergetic source having similar emission angle and pulse structure enables reductions in radiography dose by factors of 3-4x due to the narrow energy spread alone. Using multiple energies, contrast between regions of differing $\mathrm{Z}$ is drastically increased by using a monoenergetic source, by factors of a few to ten in the examples examined. Photofission dose is also reduced, with the reduction depending on the energy used: $\sim 4 \times$ at $14 \mathrm{MeV}$ where photofission is peaked, but more than $50 \times$ at the $<10 \mathrm{MeV}$ energies both appropriate to the prompt neutron signature (which is the strongest signature for the assessed cases) and allowed for cargo screening. For radiography and photofission modest energy spread at the 10\%-20\% level is desirable, and broader spreads can be used. In some photofission cases where gross number of fissions is a higher priority than dose reduction (i.e., single-sided detection), even larger energy spreads may be desirable if that increases MPS flux (as is the case for Thomson sources). Exploiting NRF signatures strongly motivates MPSs with selectable energy and with energy spreads at or below $2 \%$, with detection times and doses improving rapidly for lower energy spreads. Dose reduction compared to a bremsstrahlung source for NRF can exceed two orders of magnitude. Radiography and photofission will be possible before NRF applications, because the latter require finer control of the MPS. Application advantages are available from both pulsed beams (e.g., prompt neutron signatures) and continuous beams (e.g., easier use of counting detectors).

For many applications, intense narrowly collimated photon beams of controllable energy are a key MPS feature. In many cases, the benefits from narrow-angle emission equal or outweigh those from the monoenergetic nature of the beams. In radiography, a narrow-angle beam scanned across the target mitigates scattering contributions to image degradation. This effect was found to be dominant over photon spectrum in determining image quality, indicating that MPSs with narrower emission angle than bremsstrahlung sources will provide strongest advantage (and correspondingly, that MPSs with broader emission angles than bremsstrahlung systems may not 
be advantageous where scattering is significant). Dose reduction due to mitigation of scattering is in the range of 3-4x even compared to very narrowly collimated fan bremsstrahlung systems (and a much greater advantage vs. broadly collimated sources).

Scanning using a narrow-divergence beam also enables flux to be adapted to local attenuation at each pixel. The dose reduction obtained depends sensitively on object structure. However, realistic structures often have severe scattering and have small objects with high attenuation are surrounded by large low-density areas. In many such cases, a scanned pencil beam can reduce dose by factors ranging from a few to a hundredfold, relative to conventional fan beams, while also improving contrast. While a pencil beam would be very intense if held stationary on one position, its use reduces the number of photons required to extract signal from each area of the target, hence reducing dose required. Adapting flux while such a beam is scanned across a target can allow extraction of signal using the physics-limited minimum of dose (typically much less than used in a conventional fan beam).

Similarly, for photofission and NRF interrogation, in all application areas, use of a beam whose opening angle matches the object of interest improves signal per unit dose dramatically and in many cases is required to obtain a signal. For applications considered, appropriate beam opening angles are in the few-mrad range. Use of such narrow angle beams requires that suitable rastering systems be developed once MPS technologies are sufficiently mature.

Additional applications were identified which were addressed to the extent practical by leveraging overlap with the main topics. In particular, emergency response capabilities are indicated by the cargo, treaty and stockpile stewardship sections. The results indicate that a MPS could provide important capabilities for lower-dose radiography, for very high resolution radiography to identify components (with potential resolution as fine as micron-scale), and for secondary identification of material using NRF or photofission. As identified above, any monoenergetic source can reduce radiography dose by a factor of a few and can increase $\mathrm{Z}$ contrast (e.g., nuclear reaction sources). Realizing further dose reduction as well as high resolution and secondary identification prioritizes MPSs with adjustable energy, with narrowangle beams and with small emission spot size (e.g., Thomson sources). Repetition rate and flux could be relaxed relative to cargo applications due to lesser need for throughput. Small MPS size is a priority, which may make this a later application to be addressed. However, vehicle-mobile instruments have been mentioned in which case a nearer-term capability is realistic.

Similarly, the potential of MPSs with small photon emission spot size (e.g., Thomson sources) for high resolution radiography with reduced dose indicates strong potential impact on medical imaging, and industrial nondestructive analysis. Detailed analysis was not within the scope of this study for photonuclear data, spent fuel assembly assay, materials characterization in nuclear accident scenarios, waste drum content, nuclear fuel quality control, or pyroprocessing monitoring. These are areas where initial evaluation indicates potential for MPS benefit, and are possible topics for future work. No important capability enhancement was identified for $\mathrm{UF}_{6}$ cylinder enrichment or nuclear forensics.

Photon source requirements for prioritized and other applications appear within range of realistic MPS candidates. Two candidate technologies are under development: sources based on Thomson (also known as Compton or inverse Compton) scattering of a laser from a high energy (GeV-class) electron beam, and sources based on nuclear reactions induced in a target by a modest energy (MeV-class) ion beam. MPSs based on positron annihilation were considered previously but do not at this time present an attractive path.

Thomson photon sources produce beams of narrow (mrad) divergence and high intensity with energy, energy spread and polarization that are continuously selectable. Thomson MPSs are 
the leading technology for fixed-facility scientific sources and several are in use (e.g., HIGS). At these facilities $\mathrm{MeV}$ energies at few-percent energy spreads are routine and the beams have been used for nonproliferation signature development. Nonproliferation applications are currently limited by the need for high-energy accelerators, scattering laser systems, and associated shielding, which are large fixed facilities if conventional technology is used. For applications using $\mathrm{NRF}$, reduction of source energy spread is also important. Source development must address these limits and enable compact or transportable systems. Candidate solutions include either laser-plasma based accelerators and systems or advancement of conventional accelerators. Several development steps are required to realize compact systems producing $\mathrm{MeV}$ energies and the required beam parameters.

Demonstration of a concept for a compact source is a logical first step, addressing compact integration of a high-energy $(0.2-1 \mathrm{GeV})$ electron accelerator, scattering laser systems, and associated shielding. This demonstration can use state-of the art electron beam quality, which is sufficient to enable photon energy spreads at the 10-20\% level, appropriate to radiography and photofission. Energy of the photon beam can be adapted from shot to shot by either changing the electron accelerator energy (continuous tuning), or by frequency-converting the scattering laser (integer multiples of energy such as 3, 6, $9 \mathrm{MeV}$ ). Per-shot fluxes in the 107-108 range motivate development of techniques to more efficiently interact the laser and electron beams. Mitigation of shielding requirements may motivate novel approaches to beam disposal, one candidate for which is deceleration of the electron beam after photon production. The first development step is, therefore, demonstration that photon beams of $10-20 \%$ energy spread with fluxes in the $10^{7}$ $10^{8}$ /shot range, and at controllable $\mathrm{MeV}$ energies, could be generated by a compact system (including shielding). This would demonstrate the per-shot MPS properties required for radiography, $\mathrm{Z}$, and photofission measurements.

Demonstration of repetition rate and average flux appropriate to applications would be a logical follow-on to demonstration of a compact technology. Current Thomson sources have repetition rates at the few $\mathrm{Hz}$ level for laser-plasma based accelerators (which offer the smallest MPS sizes), and up to $\sim 100 \mathrm{~Hz}$ for conventional accelerators (higher for large rings like HIGS or large superconducting accelerators). It is anticipated that future lasers will allow $1 \mathrm{kHz}$ to potentially $200 \mathrm{kHz}$ over time, meeting application needs for fluxes in the range of 1010-1012 photons/second on target. The core laser technologies to enable high accelerator repetition rate are being developed under the Office of Science accelerator stewardship program. Modest levels of specific development directed at the scattering laser are needed. Development of $\mathrm{kHz}$ repetition rates is a logical second step for Thomson MPS development, and would demonstrate the capability to conduct measurements at acceptable rates for initial application capabilities.

For many applications, the narrow divergence of Thomson-based sources will likely require rastering of the beam across an object, which must be developed once the core MPS technology is operational. Rastering technology is a primary driver for the Screening and interdiction of SNM application, which sets the most stringent requirement: rastering across the $2.4 \mathrm{~m}$ height of a container at rates up to 80 vertical sweeps per second is desired to enable high rate scanning (somewhat lower rates would enable initial capabilities). Development of rapid rastering, together with $\mathrm{kHz}$ operation of a compact technology, would allow a complete system to be constructed that could address cargo screening. The other applications considered require pointing of the source at the tens-of seconds-scale, but not rapid rastering. This slow pointing can likely be accomplished with moderate development of existing beam pointing systems and hence rastering techniques are a critical technical element only for screening. Rastering techniques can be developed in parallel with other elements.

Further development would be required to enable advanced capabilities, and can be 
prioritized based on application selected. For both Screening and Interdiction of Shielded SNM and Detection of Hidden SNM, a key need is laser development towards repetition rates in the tens of $\mathrm{kHz}$ to realize high scan rate for radiography screening and penetration of thick targets for detection. For radiography, a key benefit of a Thomson MPS is photon dose control, which further reduces dose by delivering only the needed number of photons to a given pixel. In principle dose control can be accomplished continuously via control of the scattering laser energy. Methods to realize this control in a simple and reliable way suitable for applications are required. Exploiting NRF signatures, important to Treaty/Dismantlement Verification, Screening and Interdiction of Shielded SNM, and other applications, will require development of very fine control over the electron beam and scattering process to enable photon beams of percent-level energy spreads or lower, with correspondingly fine control of energy.

Photon emission will be in short bursts, and repetition rate must be matched to the application and detector system. For radiography and photofission this does not pose special challenges. For NRF, either high photon source repetition rates (ideally above $10 \mathrm{kHz}$ ) are needed to efficiently use conventional counting detectors such as HPGe, or alternative detection concepts are needed.

Additional signatures beyond those currently in use can be enabled by the tunable, monoenergetic, pulsed and polarized photon beam of a Thomson MPS. Possible new signatures were identified which were beyond the scope of the project to detail, and which require further signature development. Backscatter radiography or another technique appears important to constrain detection volume for Detection of Hidden SNM and could also have strong impact in resolving 3D clutter in Screening and Interdiction of Shielded SNM. This method requires a MPS with pulsed, narrow-angle emission since the beam is rastered across the target and return times and signals measured to map materials. Measurements have been made using bremsstrahlung sources, and could be improved by a pulsed narrow-angle MPS. Detailed modeling and, possibly, measurements on MPS test bed facilities are needed to quantify this capability. Other signatures for which MPSs could enable strong benefit include EZ3D-type voxelization and clutter resolution, for which a narrow-angle MPS is important (intersection of the lines determined by the photon beam and detector collimation determines resolution in this method). Realizing MPS benefit to this technique would require development of appropriate energy-resolving detectors for the pulsed MPS beam, and subsequent testing of performance on MPS test bed facilities. Reduction of scatter due to an MPS would also benefit few-view radiography and computed tomography systems, as discussed in the Stockpile Stewardship section. Compact polarized-photon MPSs could enable polarized photofission signatures (proven on fixed-facility MPSs [22]) to be used for material discrimination in Detection of Hidden SNM, Treaty/Dismantlement, or cargo secondary screening. This may enable isotope identification with $10 \%$-level energy spread instead of the percent level or lower needed for NRF. Thomson MPSs provide suitable polarized beams. As with dose control, development of MPS techniques to accomplish polarization control in a compact, robust manner are needed. MPSs with adjustable energy and narrow emission angle could also enable photoneutron spectroscopy for identification of non-nuclear materials, or fission product isomers for isotope identification. As with NRF signatures, fine control of MPS energy at the percent level is likely required to utilize this signature. Continued research on such topics at a basic signature science level is important to enable additional MPS benefit for nonproliferation applications. As MPS technology matures and more precise evaluations are needed, continued cross section and signature measurements to increase precision will also be important. Key MPS properties by application and signature for a Thomson-type source are listed in Table 28.

The principal MPS candidate other than Thomson scattering is use of nuclear reactions, including ${ }^{11} \mathrm{~B}(\mathrm{~d}, \mathrm{n} \gamma)^{12} \mathrm{C}$, which produces photons both at $15.1 \mathrm{MeV}$ and in multiple lines around 2-5 
$\mathrm{MeV}$ and neutrons. Applications are currently limited by accelerator and shielding size, and a DNDO TAR project is in progress on these sources. The gamma rays at $15.1 \mathrm{MeV}$ and at $4.4 \mathrm{MeV}$ could be exploited for Z-discrimination based on dual energy transmission. The gamma rays at $15.1 \mathrm{MeV}$ as well as the neutrons produced could be used to excite fission. Other candidate reactions are being explored. Emission is unpolarized and into $4 \pi$ angle. Designs have been proposed for systems capable of yields in the range of $10^{8}$ to (at very high current) $10^{11}$ photons/second (into $4 \pi$, not on target). It is likely that nuclear reaction based sources would be used primarily for screening and related applications, since single-sided detection, treaty and safeguards applications assessed prioritize very narrow divergence beams. The usable photon fraction would depend on the application geometry, and would likely be collimated to produce a fan beam of much larger angular spread than Thomson sources. Flux into a relatively broad fan collimator of 2 degrees by 30 degrees might be of order $10^{5-10^{8}}$ photons/second on target. Development of higher fluxes, or of scenarios where multiple collimators or broader collimation can be used (accounting for scattering effects), would be important to take advantage of such sources. The beam can be continuous or pulsed depending on the ion accelerator used.

Table 28: Key Thomson MPS performance parameters for evaluated applications. For all, few-mrad divergences are suitable. Particular challenges are underlined.

\begin{tabular}{|c|c|c|c|c|c|}
\hline Application & Energy & $\begin{array}{l}\text { Energy } \\
\text { spread }\end{array}$ & $\begin{array}{l}\text { Photons/sec, } \\
\text { Rep-rate }\end{array}$ & Rastering & Size \\
\hline $\begin{array}{l}\text { Screening - } \\
\text { Radiography, Z }\end{array}$ & $3-9$ & $10-20 \% *$ & $\begin{array}{l}10^{10}-10^{12} \\
\mathrm{kHz}-\underline{50 \mathrm{kHz}}\end{array}$ & $\begin{array}{l}\text { Rapid: up to } \\
\text { 80sweep/sec }\end{array}$ & $\begin{array}{l}\text { Building or } \\
\text { Truck }\end{array}$ \\
\hline $\begin{array}{l}\text { Screening - } \\
\text { Photofission }\end{array}$ & $6-9$ & $10-30 \%$ & $\begin{array}{l}10^{11-10^{12}} \\
\geq \mathrm{kHz}\end{array}$ & Slow & $\begin{array}{l}\text { Building or } \\
\text { Truck }\end{array}$ \\
\hline Screening- NRF & $1-7$ & $<2 \%$ & $\begin{array}{l}10^{10} \\
\geq \mathrm{kHz}\end{array}$ & Slow & $\begin{array}{l}\text { Building or } \\
\text { Truck }\end{array}$ \\
\hline $\begin{array}{l}\text { Detection - } \\
\text { Photofission }\end{array}$ & $6.5-15$ & $20-40 \%$ & $\begin{array}{l}>10^{11} \\
>\mathrm{kHz}\end{array}$ & Slow & Truck \\
\hline $\begin{array}{l}\text { Treaty/ } \\
\text { Dismantlement- } \\
\text { NRF }\end{array}$ & $1-7$ & $<2 \%$ & $\begin{array}{l}10^{10} \\
\geq 10 \mathrm{kHz}\end{array}$ & Slow & $\begin{array}{l}\text { Truck or } \\
\underline{\text { smaller }}\end{array}$ \\
\hline $\begin{array}{l}\text { Safeguards - } \\
\text { Transmission }\end{array}$ & $6-8$ & $20-30 \%$ & $10^{12}$ & Slow & Truck \\
\hline
\end{tabular}

To demonstrate and experimentally develop MPS applications, a sequence of laboratorybased application tests could be accomplished on existing fixed-facility MPSs, such as HIGS and its potential upgrade, as well as on test facilities under construction for demonstration of compact MPS technologies. As MPS technology matures, such tests could verify the predictions of simulations conducted under this project and in the literature and guide development of field 
applications. Radiography and Z discrimination tests have been conducted using both Thomson and nuclear reaction based sources. The simulations conducted here indicate that further tests should focus on configurations that strongly test MPS advantages, i.e., that are both strongly attenuating and where strong scattering contributions would degrade signals using conventional systems. To do this, simply using dense material is not sufficient. Appropriate radiography tests could include cases such as CAARS object 4, or other cases with a dense object surrounded by meter-scale areas of low-Z materials such as that shown in Figure 10 of the Screening and Interdiction of Shielded SNM section. The effect of photon beam emission angle on contrast and Z discrimination, as simulated here, could be verified using such targets. For Thomson MPSs that produce small emission spot sizes, tests of radiography resolution should be extended to thick test objects to demonstrate utility for high-resolution applications such as Stockpile/Treaty measurements or Emergency Response. Tests relevant to radiography and $\mathrm{Z}$ for cargo screening and stockpile applications can be conducted starting with the first phase of MPS development, since modest energy spreads are required, and typically one or a few shots per location are sufficient, which eases tests on low repetition rate facilities. Testing of chord transmission measurements appropriate to verification of occupancy in a dry storage cask, as shown in the 'Safeguards' section, is challenging due to the very low transmission. Tests using a mockup of a cask will therefore be most effective as high flux $\mathrm{kHz}$ Thomson sources are developed, though initial measurements may be possible at lower repetition rate. For both Photofission and NRF, proof of principle experiments have been done, and can be continued, on fixed facility MPSs and on test beds for compact MPSs. Photofission tests can be conducted on thinly shielded targets using low repetition rate facilities, as has been done to date. However, to address the integrated challenge of thickly shielded targets, such as those evaluated here for Screening and Interdiction of Shielded SNM and for Detection of Hidden SNM, high-flux facilities such as a kHz-class Thomson source will be important. NRF-based tests will prioritize both high repetition rate and narrow energy spread. In the near term, until compact technologies are developed with the required fine resolution, such tests can be continued at fixed facilities. Full evaluation of heavily shielded cases would require operation of a high flux, high repetition rate source in a facility able to handle kg-class quantities highly enriched uranium or plutonium. For the applications assessed, neither object size nor rastering is an important limit on laboratory tests. Compact MPS test facilities as well as existing fixed facilities can typically accommodate objects up to 2-3 m on a side, which is sufficient for realistic tests for all of the assessed applications. For example, while a full cargo container or fuel cask is too large to fit in these facilities, the available size is enough to construct a realistic mock-up that would provide appropriate depth, structure, attenuation, and other key parameters. Similarly, a layered target as simulated under the 'Treaty/Dismantlement Verification' section could be used to test that application without access to a weapon. Rastering can be simulated by moving the object scanned for laboratory tests.

MPS compactness is an important driver of utility and of the transition from laboratory to field tests, and requirements vary by application. For Screening and Interdiction of Shielded SNM, systems in use fit in small buildings (such as the Passport system at the Port of Boston). This size is compatible with current facilities for laser-plasma based Thomson MPSs and potentially with other technologies. It is likely that such an installation would be the logical first step for MPS field applications, once the compactness, repetition rate, and rastering development described above is accomplished. Hence compactness is not the principal development need for this application. It has been discussed that an attractive scenario may be initial installation at a nuclear facility (e.g., a national lab where shipments of nuclear material are common) where the MPS could be used to monitor vehicles entering and leaving the facility. This scenario has more modest rate requirements than commercial cargo. Installation in such a facility would also allow use of the source for other applications such as Stockpile Stewardship and for tests on realistic objects for 
Treaty/dismantlement verification and Detection of Hidden SNM. An installation similar to the Passport system at a port would also be a good pilot deployment for establishing cargo screening capabilities. Field applications of Detection of Hidden SNM, Treaty/Dismantlement Verification, and Nuclear Safeguards Dry Storage Cask Verification likely require systems at or below the size of a small truck, with correspondingly modest electrical requirements at the $60 \mathrm{kWe}$ level. This size would also be highly desirable for Screening and Interdiction of Shielded SNM. Integration of a system in this size appears realistic, and would be a logical second step for MPS field applications. Based on successful demonstration at this intermediate scale, further development to reduce size and weight to levels needed, for example, for Emergency Response would then follow. Emergency Response typically requires lower repetition rates than other applications, which may mitigate difficulty in scaling to smaller sizes.

The order in which applications can be addressed will depend on progress on each of the development tasks identified. With realization of appropriate rastering techniques for Thomson MPSs, or sufficient flux and collimator systems for nuclear reaction MPSs, Screening and Interdiction of Shielded SNM may be a logical first application because of the strong utility of MPSs of modest size (small-building) and modest energy spread (10-20\% for radiography, Z discrimination and photofission). Using Thomson sources, such a system would also enable stockpile measurements with relevance to future treaty and dismantlement applications. If lasers and/or accelerator systems make rapid progress in size, applications such as Detection of Hidden SNM and Nuclear Safeguards Dry Cask Verification, which do not place stringent requirements on rastering methods, may be suitable early applications for Thomson based sources. Applications requiring NRF require very precise control of Thomson MPS energy and energy spread, which is likely to require longer-term development. Likewise, Emergency Response applications that need very compact systems are also likely to likely to require longer-term development.

In summary, simulations showed that MPSs can offer significant benefit to cargo screening, detection, warhead verification, and nuclear fuel cask verification. They indicate that these benefits are also likely to impact emergency response and many other application areas both within and outside of nonproliferation work. Full benefit is realized with an MPS having adjustable energy in the 1.5-15 MeV range, narrow (mrad) emission angle, energy spreads from approximately $20 \%$ for radiography and photofission down to $<2 \%$ for NRF, and with photon fluxes of $10^{10-10^{12}}$ photons/second delivered to the target. These parameters match with the development outlook for Thomson/Compton based photon sources, provided that such systems can be made sufficiently compact and can operate at the required repetition rates $(\geq \mathrm{kHz})$. Early applications will prioritize radiography and photofission signatures due to their more relaxed demands on source performance compared to NRF. Such a coordinated program could deliver high-impact, next-generation systems with greatly reduced dose and increased detection and measurement capabilities. New signatures enabled by such sources may offer additional benefit. 


\section{References}

[1] C. Geddes et al., Impact of Monoenergetic Photon Sources on Nonproliferation Applications: Initial Assessment and Prioritization Report" Idaho National Laboratory, Idaho Falls, Idaho INL/LTD-15-35872, 2015.

[2] R. C. Runkle, D.L. Chichester, and S.J. Thompson, Rattling Nucleons: New Developments in Active Interrogation of Special Nuclear Material" Nucl. Instrum. Meth. A 663 (2012) 75-95.

[3] M. Chen, E. Esarey, C.G.R. Geddes, C. B. Schroeder, G. R. Plateau, S. S. Bulanov, S. Rykovanov, and W. P. Leemans, Modeling classical and quantum radiation from laser-plasma accelerators, Phys. Rev. Special Topics-Accelerators and Beams 16, 030701 (2013).

[4] B.J. Quiter et al, "A method for high-resolution x-ray imaging of intermodal cargo containers for fissionable materials," J. Appl. Phys. 103, 064910 (2008).

[5] S. Korbly, W. Bertozzi, R. Ledoux and R. Richardson, private communication.

[6] M. Johnson, et al., "Using Quasi-Monoenergetic Photon Sources To Probe Photo-Fission Resonances," AIP Conference Proceedings, Vol. 1336. Issue 1, p. 590 (2011).

[7] E. S.Cardenas, et al., Comparison of fission signatures from $\beta$-delayed $\gamma$-ray and neutron emissions, Nuclear Instruments and Methods A 792 (2015).

[8] W. Bertozzi, J.A. Caggiano, W.K. Hensley, MS Johnson, SE Korbly, RJ Ledoux, DP McNabb, EB Norman, WH Park and GA Warren, Nuclear Resonance Fluorescence Excitations Near $2 \mathrm{MeV}$ in 235U and 239Pu, Phys. Rev. C 78, 041601-5 (2008).

[9] B.J. Quiter, B.A. Ludewigt, V.V. Mozin, S.G. Prussin, Nuclear Resonance Fluorescence for Materials Assay, IEEE Transactions on Nuclear Science 58 (2) 400 (2011).

[10] B.J. Quiter, BA Ludewigt, VV Mozin, C Wilson and S Korbly, Transmission nuclear resonance fluorescence measurements of 238U in thick targets, Nucl. Instrum. Meth. B 2691130 (2011).

[11] G.A. Warren, J.A. Caggiano, W. Bertozzi, S. Korbly, R. Ledoux, and W.H. Park, On the Search for Nuclear Resonance Fluorescence Signatures of $235 \mathrm{U}$ and $238 \mathrm{U}$ above $3 \mathrm{MeV}$, IEEE Trans. Nucl. Sci. 57, 317-322 (2010)

[12] B.J. Quiter et al., Nuclear resonance fluorescence in 240Pu, Physical Review C 86, 034307 (2012).

[13] W. Bertozzi, R.J. Ledoux, Nuclear Resonance Fluorescence imaging in non-intrusive cargo inspection," Nucl. Inst. Meth. B. 241 820-825 (2005).

[14] F. Albert, S. G. Anderson, G. A. Anderson, S. M. Betts, D. J. Gibson, C. A. Hagmann, J. Hall, M. S. Johnson, M. J. Messerly, V. A. Semenov, M. Y. Shverdin, A. M. Tremaine, F. V. Hartemann, C. W. Siders, D. P. McNabb, and C. P. J. Barty, Isotope-specific detection of low-density materials with laser-based monoenergetic gamma-rays, Opt. Lett. 35, 354-356 (2010).

[15] M.S. Johnson, C.A. Hagmann, J.M. Hall, D.P. McNabb, J.H. Kelley, C. Huibregtse, E. Kwan, G. Rusev, A.P. Tonchev, Searching for illicit materials using nuclear resonance fluorescence stimulated by narrow-band photon sources, Nucl. Inst. Meth. B. 285, 15 August 2012, Pages 72-85.

[16] C.A. Hagmann et al., Journal of Applied Physics 106, 084901 (2009).

[17] B. J. Quiter, T. Laplace, and B. A. Ludewigt, Examining Pu-239 and Pu-240 Nuclear Resonance Measurements on Spent Fuel for Nuclear Safeguards, in Proc. of the Annual Meeting of the Institute for Nuclear Material Management, Orlando, Florida (2012).

[18] F. Albert et al., Phys. Rev. ST-AB, 13, 070704 (2010).

[19] R. Hajima et al., Nucl. Inst. Meth. A 608, p.557, (2009).

[20] J. Pruet, D. P. McNabb, C. A. Hagmann, F. V. Hartemann, and C. P. J. Barty, Detecting clandestine material with nuclear resonance fluorescence," Journal Of Applied Physics 99, 123102 (2006).

[21] J. M. Hall, V. A. Semenov, F. Albert, and C. P. K. Barty, Numerical simulation of nuclear materials detection, imaging and assay with MEGa-rays, Proc. 52nd Annual Meeting of the Institute of Nuclear Material Management (Palm Desert, California, 2011). 
[22] J.M. Mueller, et al., Tests of a novel method to assay SNM using polarized photofission and its sensitivity in the presence of shielding, Nucl. Inst. Meth. A 776, 107-113 (2015).

[23] A.P. Tonchev, private communication.

[24] J.E. McFee et al., Photoneutron spectroscopy using monoenergetic gamma rays for bulk explosives detection, Nucl. Inst. Meth. A 704 (2013) 131-139.

[25] H.R. Weller et al, Proc. Part. Nucl. Phys. 62, p. 257 (2009).

[26] O. Tesileanu et al., Journal of Physics: Conference Series 420 (2013).

[27] ASTA: http://asta.fnal.gov/files/ASTA_Proposal_October_2013.pdf

[28] FACETII: https://portal.slac.stanford.edu/sites/ard_public/facet/Documents/FACETII\%20Proposal\%20v6.pdf

[29] ATF: http://www.bnl.gov/atf/

[30] C.G.R. Geddes, S. Rykovanov, N.H. Matlis, S. Steinke, J.-L. Vay, E. Esarey, B. Ludewigt, K. Nakamura, B.J. Quiter, C.B. Schroeder, C. Toth, W.P. Leemans, Compact quasi-monoenergetic photon sources from laserplasma accelerators for nuclear detection and characterization," Nucl. Inst. Meth. B. 350, 116 (2015).

[31] H. Schwoerer, B. Liesfeld, H.-P. Schlenvoigt, K.-U. Amthor, and R. Sauerbrey, Thomson-Backscattered X Rays From Laser-Accelerated Electrons, Phys. Rev. Letters 96 (2006) 014802.

[32] K. Ta Phuoc, S. Corde, C. Thaury, V. Malka, A. Tafzi, J. P. Goddet, R. C. Shah, S. Sebban, A. Rousse, Alloptical Compton gamma-ray source, Nature Photonics 6 (2012) 308. Similar exp. at U. Michigan.

[33] S. Chen, N. D. Powers, I. Ghebregziabher, C. M. Maharjan, C. Liu, G. Golovin, S. Banerjee, J. Zhang, N. Cunningham, A. Moorti, S. Clarke, S. Pozzi, D. P. Umstadter, MeV-Energy X Rays from Inverse Compton Scattering with Laser-Wakefield Accelerated Electrons," Phys. Rev. Lett. 110, (2013) 155003. Similar exp. at MPQ.

[34] S. Chen, G. Golovin, C. Miller, D. Haden, S. Banerjee, P. Zhang, C. Liu, J. Zhang, B. Zhao, S. Clarke, S. Pozzi, D. Umstadter, Shielded radiography with a laser-driven MeV-energy X-ray source, Nucl. Inst. Meth. B 366, pp. 217-223 (2016).

[35] C.P.J. Barty, Advanced Laser-Compton Gamma-Ray Sources for Nuclear Materials Detection, Assay and Imaging," in Proceedings of the International Symposium on Nuclear Physics and Gamma-Ray Sources for Nuclear Security and Nonproliferation, pp 85-95 (2014).

[36] Barty, C. P. J., F. Albert, S. G. Anderson, P. Armstrong, A. Bayramian, G. Beer, R. Cross, C. Ebbers, G. Deis, D. J. Gibson, J. Hall, F. V. Hartemann, T. L. Houck, R. A. Marsh, M. J. Messerly, H. Phan, V. Semenov and S. S.

Q. Wu. Overview of MEGa-ray-based Nuclear Materials Management Activities at the Lawrence Livermore National Laboratory, in Proceedings of the 52nd Annual Meeting of the Institute for Nuclear Materials Management, Vol 3, pp. 2645-2654 (2011).

[37] W.P. Leemans et al., Phys. Rev. Lett. 77, p. 4182 (1996).

[38] A. Jochmann et al., Phys. Rev. Lett. 111, 114803 (2013).

[39] Example: http://www.physikinstrumente.com/product-detail-page/s-334-300710.html

[40] T. Hosokai et al., "Transient magnetized plasma as an optical element for high power laser pulses," Physical Review Special Topics-Accelerators and Beams 18, 021303 (2015).

[41] T.J.T. Kwan et al., 'The development of enabling technologies for producing active interrogation beams," Rev. Sci. Instrum. 81, 1033034 (2010).

[42] A.J. Antolak et al., Active interrogation using low-energy nuclear reactions, Proceedings of SPIE: Penetrating Radiation Systems and Applications V, F. Patrick Doty, H. Bradford Barber, Han Roehrig, Eds., (August 2005).

[43] T.N. Taddeucci and R.L. Sheffield, "Neutron and gamma-ray production with low-energy beams," Los Alamos National Laboratory Report LA-UR-07-2724 (2010).

[44] B. O'Day, Z.S. Hartwig, R.C. Lanza, A. Danagoulian, Initial results from a multiple monoenergetic gamma radiography system for nuclear security, Nucl. Instrum. Meth. A 832, pp. 68-76 (2016). 
[45] P. Rose, A. Erickson, M. Mayer, J. Nattress, and I. Jovanovic, “Uncovering Special Nuclear Materials by Low-energy Nuclear Reaction Imaging”, Scientific Reports 6, 24388 (2016).

[46] Warburton et al, Physical Review Volume 128, Number 4, November 15, 1962.

[47] C.R. Hatcher et al, "Width of the photon line produced by positron annihilation at 15 MeV," Nuclear Instruments and Methods 14, 337-342 (1961).

[48] L.W. Campbell, J.E. Fast, R.S. Wittman, R.J. Abrams, A. Afanasev, C. Ankenbrandt, K. Beard, G. Flanagan, RP Johnson, C Yoshikawa, and M Popovic. Monoenergetic Photon Beams based on Positron Annihilation, Pacific Northwest National Laboratory, Richland, WA, PNNL-22882 (2013).

[49] Glossary, Safeguards 2001 edition, International nuclear verification series no. 3.

[50] Medalia, Detection of Nuclear weapons and materials: Science, technologies, observations, Congressional Research service R40154 (2009).

[51] https://www.fbo.gov FY12 ATD BAA - NRIP Amended Apr 4 2012.pdf HSHQDC-12-R-00035.

[52] Descalle et al, LLNL Report No. UCRL-TR-225780 (2006).

[53] Miller et al., Applied Radiation and Isotopes 69 (2011) 594-603.

[54] White et al., Nucl. Instrum. Meth. B 266 (2008) 2079-2089.

[55] https://www.leidos.com/products/security/mobile-vacis

[56] http://www.rapiscansystems.com/en/products/cvi

[57] http://as-e.com/products-solutions/cargo-vehicle-inspection

[58] S. Ogorodnikov, V. Petrunin, Processing of interlaced images in 4-10 MV dual energy customer system for material recognition, Physics Review Special Topics 5 (2002) 104701.

[59] G. Chen et al.. Nucl. Instrum. Meth B 261 (2007) 356-359, doi:10.1016/j.nimb.2007.04.036.

[60] Cargo Advanced Automated Radiography System (CAARS) Final Report, DNDO, 600-CAARS-115930v1.00, FOUO 2011

[61] www.passportsystems.com

[62] SNAR ATD Technology Demonstration \& Characterization (TD\&C) Final Report for the Multi-Modal Automated Resolution, Location, \& Identification of Nuclear Material (MARLIN) System, DNDO, MARLIN_Final_Report_FOUO_v1.00_04_16_2013.

[63] Borozdin K., et al. ,Nature 422, 277 (20 March 2003) | doi:10.1038/422277a

[64] Slaughter et al, Nucl. Instrum. Meth. A 579, (2007) 349-352.

[65] Jordan and Gozani, Nucl. Instrum. Meth. B 261 (2007) 365.

[66] Gozani, et al, IEEE Transactions on Nuclear Science NS-60 (2013) 1118.

[67] http://physics.nist.gov/PhysRefData/Xcom/html/xcom1.html

[68] Martz H. et al., Radiography techniques to detect shielded SNM overview, presentation at SORMA West (2016).

[69] R. Richardson, private communication.

[70] O’Day, B. et al. 'Initial results from a multiple monoenergetic gamma radiography system for nuclear security' NIMA (2016) 832, 68-76 dx.doi.org/10.1016/j.nima.2016.05.117

[71] Cargo Advanced Automated Radiography System (CAARS) Final Report, DNDO, 600-CAARS-115930v1.00, FOUO 2011.

[72] Aufderheide, M. et al., HADES: a code for simulating a variety of radiographic techniques, UCRL-PROC207617. DOI: 10.1109/NSSMIC.2004.1462780.

[73] Slaughter, D. R. et al., Preliminary results utilizing high-energy fission product $\gamma$-rays to detect fissionable material in cargo, Nucl. Instrum. Meth. B 241 (2005).

[74] Thompson, S. J. et al., Utilization of high-energy neutrons for the detection of fissionable materials, Applied Physics Letters 90 (2007).

[75] Procter et al., Detecting fissionable materials in a variety of shielding matrices via delayed gamma and neutron photofission signatures, Nucl. Instrum. Meth. A 662 (2012).

[76] Bertozzi et al., Nucl. Instrum. Meth. B 261 (2007) 331-336. 
[77] E. Kwan et al., Discrete deexitations in $235 \mathrm{U}$ below $3 \mathrm{MeV}$ from nuclear resonance fluorescence, Phys. Rev. C 83, 041601 (2011).

[78] Carrel, F., et al., Detection of high-energy delayed gammas for nuclear waste packages characterization, Nucl. Instrum. Meth. A 652 (2011).

[79] Stevenson, J., et al., Linac based photofission inspection system employing novel detection concepts, Nucl. Instrum. Meth. A 652 (2011).

[80] Goldberg, M. B., et al., A Dual Purpose lon-Accelerator for Nuclear-Reaction-Based Explosives and SNMDetection in Massive Cargo, arXiv:1001.3255 (2010).

[81] Micklich, B. J., et al., FIGARO: detecting nuclear materials using high-energy gamma-rays, Nucl. Instrum. Meth. A 505 (2003).

[82] Curie, L.A., Limits for qualitative detection and quantitative determination. Application to radiochemistry, Analytical Chemistry 40 (1968).

[83] Reedy, E. T. E. et al., The detection of delayed $\gamma$-rays between intense bremsstrahlung pulses for discriminating fissionable from non-fissionable materials, Nuclear Instruments and Methods A 606 (2009).

[84] Slaughter, D. R. et al., Preliminary results utilizing high-energy fission product $\gamma$-rays to detect fissionable material in cargo, Nucl. Instrum. Meth. B 241 (2005).

[85] Slaughter, D.R., et al., Detection of special nuclear materials in cargo containers using neutron interrogation, Lawrence Livermore National Laboratory Report UCRL-ID-155315 (2003).

[86] Verberke, J.M. et al., Simulation of Neutron and Gamma-ray Emission from Fission and Photofission, Lawrence Livermore National Laboratory, May 11, 2010, UCRL-AR-228518.

[87] Mihalczo, J.T., et al., Subcriticality Measurements with HEU (93.2) Metal Annular Storage Castings, Oak Ridge National Laboratory Report ORNL/TM-2007/134 (2007).

[88] S G Rykovanov, C G R Geddes, J-L Vay, C B Schroeder, E Esarey and W P Leemans, Quasi-monoenergetic femtosecond photon sources from Thomson Scattering using laser plasma accelerators and plasma channels, Journal of Physics B 47, 23.

[89] R. Neibert, J. Zabriskie, C. Knight, and J. L. Jones, Passive and Active Radiation Measurements Capability at the INL Zero Power Physics Reactor (ZPPR) Facility," Idaho National Laboratory (2010).

[90] Glen Warren et al., Concepts for the Measurements Subsystems of the Third Generation Attributes Measurement System" Institute of Nuclear Materials Management 53rd Annual Meeting, Orlando, Florida, 15-19, July 2012.

[91] PMAC Requirements Document, Portal Monitor for Arms Control Project, Los Alamos National Laboratory, May 5, 2015.

[92] C. A. Miller, B. Ludewigt, B. J. Quiter, S. A. Pozzi, and C. G. R. Geddes, Assessing Impact of Monoenergetic Photon Sources on Nonproliferation Applications, in American Nuclear Society Advances in Nuclear Nonproliferation Technology and Policy Conference, Sante Fe, New Mexico, 2016.

[93] J. Allison et al., Geant4 developments and applications, IEEE Trans. Nucl Sci. 53 (2006) pp. 270-278.

[94] S. Agostinelli et al., GEANT4-a simulation toolkit, Nucl. Instrum. Meth. A 506 (July 2003), pp. 250-303.

[95] P. A. Hausladen, P. R. Bingham, J. S. Neal, J. A. Mullens, and J. T. Mihalczo, Portable fast-neutron radiography with the nuclear materials identification system for fissile material transfers, Nucl. Instrum. Meth. B 261 (August 2007), pp. 387-390.

[96] P. A. Hausladen et al., Induced-Fission Imaging of Nuclear Material, in Annual Meeting of the Institute of Nuclear Materials Management, Baltimore, Maryland, 2010.

[97] D. G. Langner, N. Ensslin, M. S. Krick, H. O. Menlove, M. C. Miller, and J. E. Stewart, Advances in nondestructive assay using neutron coincidence counting techniques, Abstr Pap Am Chem S 211, pp. 66NUCL, Mar 241996.

[98] S. J. Luke, G. K. White, D. E. Archer, J. K. J. Wolford, and T. B. Gosnell, Verfication of the Presence of Weapons-Quality Plutonium in Sealed Storage Containers for the Trilaterial Initiative Demonstration, Internation Atomic Energy Agency, 2001. 
[99] G. A. Warren, D. E. Archer, M. Cunningham, S. McConchie, and J. Thron, Concepts for the Measurements Subsystems of the Third Generation Attributes Measurement System, in Annual Meeting of the Institute of Nuclear Materials Management, Orlando, Florida, 2012.

[100] D. Reilly, N. Ensslin, and H. Smith, Jr., Passive Nondestructive Assay of Nuclear Materials, Los Alamos National Laboratory, Los Alamos, New Mexico, March 1991.

[101] A. L. Siedenstrang et al., PINS Chemical Assay System, User's Manual, v. 4.6, Idaho National Laboratory, 2008.

[102] A.H. Johnston, Radiation Damage of Electronic and Optoelectronic Devices in Space, presented at the $4^{\text {th }}$ International Workshop on Radiation Effects on Semiconductor Devices for Space Applications, Tsukuba, Japan, 2000.

[103] M. Smith, Project Review Meeting with Office of Nuclear Verification, Washington D.C.: Cameron Geddes, 2005.

[104] A CAT Scanner for Nuclear Weapons Components, Science and Technology Review, pp. 12-17, July/August 2009.

[105] G. F. Knoll, Radiation Detection and Measurements, 4th ed. New York: John Wiley, 2010.

[106] American National Standard for Determination of the Imaging Performance of X-Ray and Gamma-Ray Systems for Cargo and Vehicle Security Screening, American National Standards Institute N42.46-2008, 2008.

[107] P. C. Johns and M. Yaffe, Scattered radiation in fan beam imaging systems, Med Phys 9, pp. 231-239, 1982.

[108] T. M. Buzug, Computed Tomography: From Photon Statistics to Modern Cone-Beam CT. Berlin, Germany: Springer-Verlag, 2008.

[109] Mark Abhold et al., Incorporation of New Nondestructive Assay Systems into Safeguards Approaches for Spent Nuclear Fuel Assemblies, SAND2013-0240 P, 2013.

[110] Johnson, E.R., Notz, K. J., Shipping and Storage Cask Data for Spent Nuclear Fuel, ORNL/TM-11008, 1988.

[111] Wharton, C. J. et al., Summary Report: INLCDCIS Cask Scanner Testing at Doel, Belgium, INL/EXT-13-28809, 2013.

[112] M. Durham, et al., Muon monitoring of a fuel cask at the Idaho National Laboratory, LA-UR-15-23710, 2015.

[113] O.W. Hermann, R.M. Westfall, “Origen-S: Scale System Module to Calculate Fuel Depletion...," ORNL/NUREG/CSD-2/V2/R6. Oak Ridge National Laboratory, Sep. 1998.

[114] D.B. Pelowitz, ed. MCNPX Manual, Version 2.7.0. LA-CP-11-00438. Los Alamos National Laboratory, April 2011.

[115] J. Buongiorno, PWR Description, 22.06: Engineering of Nuclear Systems. Massachusetts Institute of Technology, 2010.

[116] E.R. Johnson, K.J. Notz, Shipping and Storage Cask Data for Spent Nuclear Fuel, ORNL/TM-11008. Oak Ridge National Laboratory, Nov. 1988.

[117] A. McClure, Estimation of the Westinghouse 17x17 MOX SNF Assembly Weight, A000000000-0 17 17-02 IO00001 REV 00, CRWMS/M\&O.

[118] NIST Standard Reference Database 8 (XGAM) M.J. Berger, et. al. NIST, PML, Radiation Physics Division, 1998.

[119] Mark Abhold et al., Incorporation of New Nondestructive Assay Systems into Safeguards Approaches for Spent Nuclear Fuel Assemblies, SAND2013-0240 P, 2013.

[120] M.A. Humphrey et al., The Next Generation Safeguards Initiative's Spent Fuel Nondestructive Assay Project, Journal of Nuclear Materials Management, Vol. XL, No. 3, 2012, and other articles in this volume.

[121] J. A. Kulisek, K. K. Anderson, A. M. Casella, C. J. Gesh, and G. A. Warren, Assaying Used Nuclear Fuel Assemblies Using Lead Slowing-Down Spectroscopy and Singular Value Decomposition, IEEE Trans. Nucl. Sci. 60, pp. 539-544, 2013.

[122] M.S. Johnson et al., Searching for illicit materials using nuclear resonance fluorescence stimulated by narrow-band photon source, Nucl. Instrum. Meth. B 285, 72-85, 2012. 
[123] P.M Rinard and G.E. Bosler, Safeguarding LWR Spent Fuel with the FORK Detector, Los Alamos National Laboratory, LA-11096-MS, (March 1988).

[124] Y. Ham, et. al., Partial Defect Tester (PDET) - A Novel Approach to Detect Partial Defects in Pressurized Water Reactor Spent Fuel, Nuclear Technology, August 2011.

[125] S. Jacobsson Svard, et al., Tomography for partial defect verification -experiences from measurements using different devices, ESARDA Bulletin no. 33.

[126] B.J. Quiter et al., Nuclear resonances fluorescence in ${ }^{240} \mathrm{Pu}$, Physical Review C 86, 034307 (2012).

[127] Bolind, A., in the Proceedings of the International Symposium on Nuclear Physics and Gamma-ray Sources for Nuclear Security and Nonproliferation, January 2014, p. 161-169, World Scientific, 2015, ISBN 978-9814635-44-8.

[128] Bolind, A. and Seya, M. Technical Report, Japan Atomic Energy Agency, Tokai, Ibaraki, Japan (2014).

[129] Strebentz and Chichester, Further Evaluation of the Neutron Resonance Transmission Analysis (NRTA) Technique for Assaying Plutonium in Spent Fuel, INL/EXT-11-23391, 2011.

[130] Takehito Hayakawa, et el., Nuclear Instruments and Methods in Physics Research A621 (2010) 695-700

[131] K.M.Goff, et al., Dry Processing of Used Nuclear Fuel, Proceedings of Global 2009, Paris, France, September 6-11, 2009.

[132] Won II Ko, et al., Preliminary conceptual design and cost estimation for Korea Advanced Pyroprocessing Facility Plus (KAF+), Nuclear Engineering and Design 227 (2014) 212-224.

[133] Tadashi Inoue, Lothar Koch, Development of Pyroprocessing and its Future Direction, Nuclear Engineering and Technology, Vol. 40, No. 3, 183-190, April 2008.

[134] P.C. Durst et al., Advanced Safeguard Approaches for New TRU Fuel Fabrication Facilities, PNNL_17151, 2007.

[135] L.E. Smith et al., An Unattended Verification Station for UF6 Cylinders: Implementation Concepts and Development Status, UCVS IAEA Symposium, 2014.

[136] D.V. Jordan et al., PNNL-21263, 2012.

[137] H.O. Menlove et al., A more accurate and penetrating method to measure the enrichment and mass of UF6 in storage cylinders using passive neutron self-interrogation, Proceedings INMM Annual Meeting 2010.

[138] K.A. Miller et al., Technical assessment of the passive neutron enrichment meter (PNEM) for an unattended cylinder verification station (UCVS), LA UR 13-22911, Los Alamos National Laboratory, 2013.

[139] K. Zhao, et al., IAEA STR-368, IAEA Department of Safeguards, 2010.

[140] S.B. Ludwigt et al., Revised Conceptual Designs for the FMDP MOX Fresh Fuel Transport Package, ORNL/TM-13574, 1998.

[141] C.P.J. Barty, Advanced Laser-Compton Gamma-Ray Sources for Nuclear Materials Detection, Assay, and Imaging, in Proceedings of the Int. Symp. Nuclear Physics and Gamma-Ray Sources for Nuclear Security and Nonproliferation, Ricotti, Tokai-mura, Japan, January 2014, World Scientific Publishing.

[142] M.S. Johnson et al., Applications of Photonuclear Physics for International Safeguards and Security, LLNLPROC-428233, 2010.

[143] P.G. Thirolf et al., EPJ Web of Conferences 38,08001 (2012). 